Supporting Information

\title{
Room-Temperature meta-Functionalization: Pd(II)-Catalyzed Synthesis of 1,3,5-trialkenyl Arene and meta-Hydroxylated Olefin
}

Milan Bera,* Santosh K. Sahoo, and Debabrata Maiti*

Department of Chemistry, Indian Institute of Technology Bombay, Powai, Mumbai-400076, India

Email for D.M.: dmaiti@chem.iitb.ac.in

Email for M.B.: miluom@gmail.com 


\section{General Consideration:}

Reagent Information. Unless otherwise stated, all reactions were carried out under air atmosphere in screw cap reaction tubes. All solvents were bought from Merck and TCI [1,1,1,3,3,3-Hexafluoro-2-propanol (HFIP); CAS: 920-66-1] in a sure-seal bottle and were used as received. Silver carbonate, all MPAA ligands were purchased from Alfa Aeser. Diethyl benzyl phosphonates for the synthesis of final starting materials were obtained from Aldrich and Alfa Aesar. Olefins were obtained from Alfa Aeser, Sigma Aldrich and Spectrochem. For column chromatography, silica gel (100-200 mesh) obtained from SRL Co. was used. A gradient elution using pet ether and ethyl acetate was performed, based on Merck aluminum TLC sheets (silica gel $60 \mathrm{~F}_{254}$ ).

Analytical Information. All isolated compounds were characterized by ${ }^{1} \mathrm{H},{ }^{13} \mathrm{C}$, and in few cases 1D-NOE, NOESY, HSQC NMR spectroscopy, IR spectroscopy, Gas chromatography mass spectra (GC-MS)/HR-MS for some compounds. Copies of NMR files can be found in the supporting information. Unless otherwise stated, all Nuclear Magnetic Resonance spectra were recorded on a Bruker 400 and $500 \mathrm{MHz}$ instrument. ${ }^{31} \mathrm{P}-\mathrm{NMR}$ spectra reported in the supporting information was recorded in Bruker $500 \mathrm{MHz}$ instrument. The references used for the NMR are tetramethylsilane (TMS) for ${ }^{1} \mathrm{H}$ and ${ }^{13} \mathrm{C}-\mathrm{NMR}$. All ${ }^{1} \mathrm{H}-\mathrm{NMR}$ experiments are reported in units, parts per million (ppm), and were measured relative to the signals for residual chloroform (7.26 $\mathrm{ppm})$ in the deuterated solvent, unless otherwise stated. All ${ }^{13} \mathrm{C}-\mathrm{NMR}$ spectra were reported in ppm relative to deuterochloroform $(77.23 \mathrm{ppm})$, unless otherwise stated, and all were obtained with ${ }^{1} \mathrm{H}$ decoupling. Neat infrared spectra were recorded on a Perkin-Elmer spectrum one FT-IR spectrometer. The data was recorded in transmittance mode $\left(\% \mathrm{~T}, \mathrm{~cm}^{-1}\right)$.-The melting points were measured in Büchi Melting Point Model B-545 apparatus. All NMR analysis were performed 1,3,5-trimthoxybenzene as the internal standard. High-resolution mass spectra (HRMS) were recorded on a micro-mass ESI TOF (time of flight) mass spectrometer.

\section{Description of Reaction Tube:}
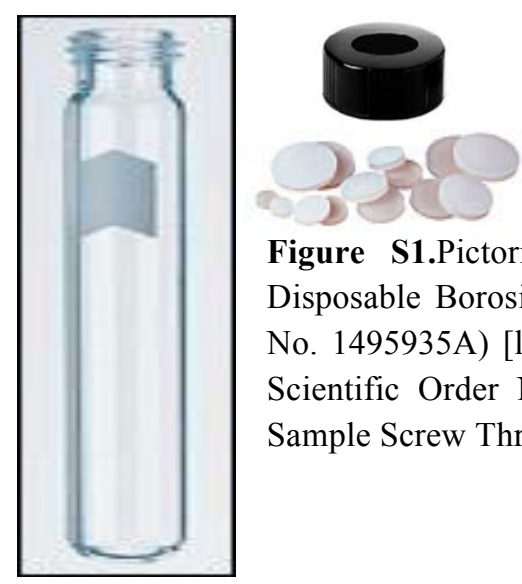

Figure S1.Pictorial description of reaction tube for meta-functionalizations: Fisherbrand Disposable Borosilicate Glass Tubes $\left(16^{*} 125 \mathrm{~mm}\right)$ with Threaded End (Fisher Scientific Order No. 1495935A) [left]; Kimble Black Phenolic Screw Thread Closures with Open Tops (Fisher Scientific Order No. 033407E) [right]; Thermo Scientific National PTFE/Silicone Septa for Sample Screw Thread Caps (Fisher Scientific Order No. 03394A) [right]. 


\section{Optimization details for Activation of Remote Meta-C-H Bonds:}

\section{Optimization for mono-olefination}

\section{Temperature Screening:}<smiles>C=CC(=O)OCC</smiles>

$0.1 \mathrm{mmol}$<smiles>CCOCC=Cc1cc(C=CCOCC)cc(CP(=O)(OCC)Oc2ccccc2C#N)c1</smiles>

Scheme S1: Temperature screening

Table S1:

\begin{tabular}{|c|c|c|c|c|}
\hline entry & Temperature $\left({ }^{\circ} \mathrm{C}\right)$ & $\begin{array}{c}\text { Mono-product } \\
(\mathbf{\%})\end{array}$ & Di-product (\%) & $\begin{array}{c}\text { Selectivity } \\
\text { meta: } \text { others })\end{array}$ \\
\hline 1 & $30(\mathbf{R T})$ & 74 & 11 & $>20: 1$ \\
\hline 2 & 50 & 55 & 32 & $>20: 1$ \\
\hline 3 & 80 & 10 & 83 & $>20: 1$ \\
\hline 4 & 90 & 10 & 81 & $>20: 1$ \\
\hline 5 & 100 & 8 & 79 & $>20: 1$ \\
\hline
\end{tabular}

Yield and selectivity were determined based on ${ }^{1} \mathrm{H}$ NMR of crude reaction mixture using 1,3,5-trimethoxybenzene as an internal standard.

\section{Oxidant Screening:}<smiles>C=CC(=O)OCC</smiles>

$0.1 \mathrm{mmol}$

$0.2 \mathrm{mmol}$
$\mathrm{Pd}(\mathrm{OAc})_{2}(10 \%)$

Ac-Gly-OH (20\%)

Oxidant (3 equiv)

HFIP ( $0.7 \mathrm{~mL}$ ), RT

$24 \mathrm{~h}$

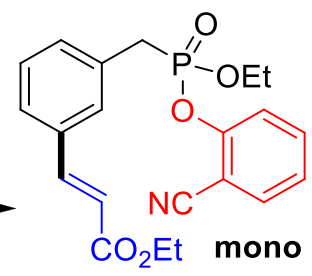<smiles>CCOCC=Cc1cc(C=CC(=O)OCC)cc(CP(=O)(OCC)Oc2ccccc2C#N)c1</smiles>

Scheme S2: Oxidant screening

Table S2:

\begin{tabular}{|c|c|c|c|c|}
\hline entry & Oxidant & $\begin{array}{c}\text { Mono-product } \\
(\%)\end{array}$ & Di-product (\%) & $\begin{array}{c}\text { Selectivity } \\
\text { (meta: others) }\end{array}$ \\
\hline 1 & - & 0 & 0 & - \\
\hline 2 & $\mathrm{AgOAc}$ & 71 & 8 & $>20: 1$ \\
\hline 3 & $\mathrm{Ag}_{2} \mathrm{CO}_{3}$ & 74 & $\mathbf{1 1}$ & $>\mathbf{2 0 : 1}$ \\
\hline 4 & $\mathrm{AgNO}_{3}$ & 52 & 7 & $>20: 1$ \\
\hline 5 & $\mathrm{Ag}_{2} \mathrm{SO}_{4}$ & 53 & 6 & $>20: 1$ \\
\hline 5 & $\mathrm{Cu}(\mathrm{OAc})_{2}$ & - & - & - \\
\hline 6 & $\mathrm{CuCl}_{2}$ & - & - & - \\
\hline
\end{tabular}




\begin{tabular}{|c|c|c|c|c|}
\hline 7 & $\mathrm{PhI}(\mathrm{OAc})_{2}$ & - & trace & - \\
\hline 8 & $\mathrm{~K}_{2} \mathrm{~S}_{2} \mathrm{O}_{8}$ & - & trace & - \\
\hline
\end{tabular}

Yield and selectivity were determined based on ${ }^{1} \mathrm{H}$ NMR of crude reaction mixture using 1,3,5-trimethoxybenzene as an internal standard.

\section{Optimization of oxidant loading:}<smiles>C=CC(=O)OCC</smiles>

$0.1 \mathrm{mmol}$

$0.2 \mathrm{mmol}$
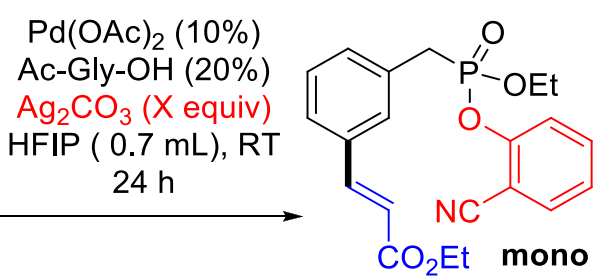

Scheme S3: Oxidant loading screening

Table S3:

\begin{tabular}{|c|c|c|c|c|}
\hline entry & $\mathrm{Ag}_{2} \mathrm{CO}_{3}$ (X equiv) & $\begin{array}{c}\text { Mono-product } \\
(\%)\end{array}$ & Di-product (\%) & $\begin{array}{c}\text { Selectivity } \\
\text { (meta: } \text { others) }\end{array}$ \\
\hline 1 & 1 & 60 & 8 & $>20: 1$ \\
\hline 2 & 1.5 & 67 & 7 & $>20: 1$ \\
\hline 3 & 2 & 79 & 11 & $>\mathbf{2 0}: 1$ \\
\hline 4 & 2.5 & 78 & 11 & $>20: 1$ \\
\hline 5 & 3 & 74 & 13 & $>20: 1$ \\
\hline 7 & 4 & 74 & $>15: 1$ \\
\hline
\end{tabular}

Yield and selectivity were determined based on ${ }^{1} \mathrm{H}$ NMR of crude reaction mixture using 1,3,5-trimethoxybenzene as an internal standard.

\section{Ligand Screening:}<smiles>C=CC(=O)OCC</smiles>

$0.1 \mathrm{mmol}$

$0.2 \mathrm{mmol}$

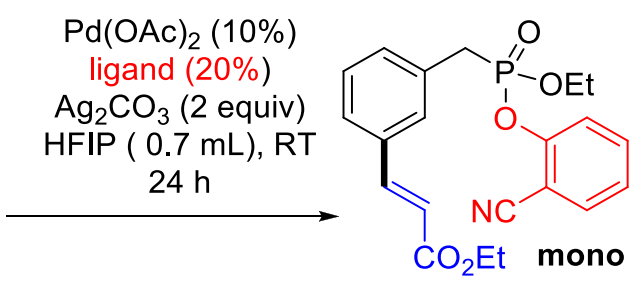<smiles>CCOCC=Cc1cc(C=CC(=O)OCC)cc(CP(=O)(OCC)Oc2ccccc2C#N)c1</smiles>

Scheme S4: Ligand screening

Table S4:

\begin{tabular}{|c|c|c|c|c|}
\hline entry & Ligand & Mono-product (\%) & Di-product (\%) & $\begin{array}{c}\text { Selectivity (meta } \\
: \text { others) }\end{array}$ \\
\hline 1 & - & 37 & 0 & $>20: 1$ \\
\hline 2 & $\begin{array}{c}\text { N-Acetyl-L- } \\
\text { phenylalanine (Ac-Phe- } \\
\text { OH) }\end{array}$ & $\mathbf{8 1}$ & $\mathbf{0}$ & $>\mathbf{2 0 : 1}$ \\
\hline 3 & $N$-Acetyl-DL-valine & 56 & 0 & $>20: 1$ \\
\hline 4 & $N$-Acetylglycine & 79 & 6 & $>20: 1$ \\
\hline 5 & $N$-Acetylalanine & 77 & 0 & $>20: 1$ \\
\hline
\end{tabular}




\begin{tabular}{|c|c|c|c|c|}
\hline 6 & $N$-Acetyl-L-leucine & 63 & 0 & $>20: 1$ \\
\hline 7 & N-Boc-D-valine & 62 & 8 & $>20: 1$ \\
\hline 8 & $D L$-Proline & 5 & 0 & - \\
\hline 9 & $N$-Boc proline & 4 & 0 & - \\
\hline 10 & 1,10 -Phenanthroline. $\mathrm{H}_{2} \mathrm{O}$ & - & - & - \\
\hline 11 & $\begin{array}{c}3,4,7,8-\text {-Tetramethyl-1,10- } \\
\text { phenanthroline }\end{array}$ & - & - & - \\
\hline 12 & Racemic-BINAM & - & - & - \\
\hline 13 & R-BINAM & - & - & \\
\hline
\end{tabular}

Yield and selectivity were determined based on ${ }^{1} \mathrm{H}$ NMR of crude reaction mixture using 1,3,5-trimethoxybenzene as an internal standard.

\section{Time Screening:}<smiles>C=CCOCCOC(=O)Cc1ccccc1</smiles>

$0.1 \mathrm{mmol}$

$0.2 \mathrm{mmol}$

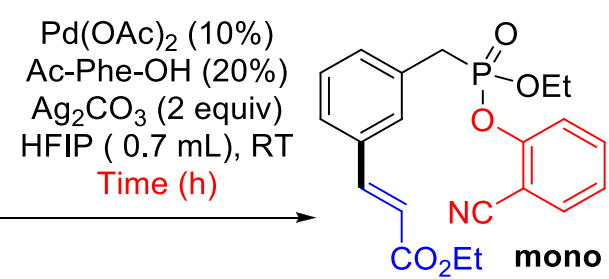

Scheme S5: Time screening<smiles>CCOCC=Cc1cc(C=CCOCC)cc(CP(=O)(OCC)Oc2ccccc2C#N)c1</smiles>

Table S5:

\begin{tabular}{|c|c|c|c|c|}
\hline entry & Time (h) & $\begin{array}{c}\text { Mono-product } \\
(\mathbf{\%})\end{array}$ & Di-product (\%) & $\begin{array}{c}\text { Selectivity } \\
\text { (meta: others) }\end{array}$ \\
\hline 1 & 18 & 74 & 0 & $>20: 1$ \\
\hline 2 & 24 & 81 & 0 & $>20: 1$ \\
\hline 3 & 30 & 83 & 0 & $>20: 1$ \\
\hline 4 & 36 & $\mathbf{8 7}$ & $\mathbf{0}$ & $>\mathbf{2 0}: 1$ \\
\hline 5 & 42 & 86 & 4 & $>20: 1$ \\
\hline 6 & 48 & 86 & 8 & $>20: 1$ \\
\hline
\end{tabular}

Yield and selectivity were determined based on ${ }^{1} \mathrm{H}$ NMR of crude reaction mixture using 1,3,5-trimethoxybenzene as an internal standard.

\section{Optimization of di-olefination: (ligand screening)}<smiles>C=CC(=O)OCC</smiles>

$0.1 \mathrm{mmol}$

$0.2 \mathrm{mmol}$

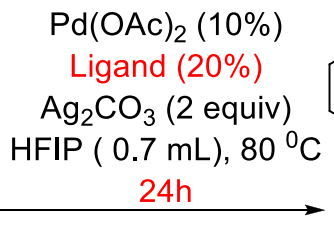<smiles>CCOC(=O)C=Cc1cccc(CP(=O)(OCC)Oc2ccccc2C#N)c1</smiles><smiles>CCOCC=Cc1cc(C=CC(=O)OCC)cc(CP(=O)(OCC)Oc2ccccc2C#N)c1</smiles>

Scheme S6: Ligand screening

Table S6:

\begin{tabular}{|c|c|c|c|c|}
\hline entry & Ligand & Mono-product (\%) & Di-product (\%) & $\begin{array}{c}\text { Selectivity (meta } \\
\text { others) }\end{array}$ \\
\hline
\end{tabular}




\begin{tabular}{|c|c|c|c|c|}
\hline 1 & $N$-Acetyl-L-phenylalanine & 12 & 78 & $>20: 1$ \\
\hline 2 & $N$-Acetyl-DL-valine & 11 & 47 & $>20: 1$ \\
\hline 3 & $\begin{array}{c}N \text {-Acetylglycine (Ac-Gly- } \\
\text { OH) }\end{array}$ & 5 & 88 & $>20: 1$ \\
\hline 4 & $N$-Acetylalanine & 17 & 72 & $>20: 1$ \\
\hline 5 & $N$-Acetyl-L-leucine & 19 & 49 & $>20: 1$ \\
\hline 6 & N-Boc-D-valine & 24 & 56 & $>20: 1$ \\
\hline 7 & $D L$-Proline & 12 & 0 & - \\
\hline 8 & $N$-Boc proline & - & - & - \\
\hline 9 & 1,10-Phenanthroline. $\mathrm{H}_{2} \mathrm{O}$ & - & - & - \\
\hline 10 & $\begin{array}{c}\text { 3,4,7,8-Tetramethyl-1,10- } \\
\text { phenanthroline }\end{array}$ & - & - & - \\
\hline 11 & Racemic-BINAM & - & - & - \\
\hline 12 & R-BINAM & - & - & - \\
\hline
\end{tabular}

Olefination with MBM scaffolds:<smiles>CCOP(=O)(Cc1ccccc1)Oc1ccc(OC)cc1C#N</smiles>

$0.1 \mathrm{mmol}$

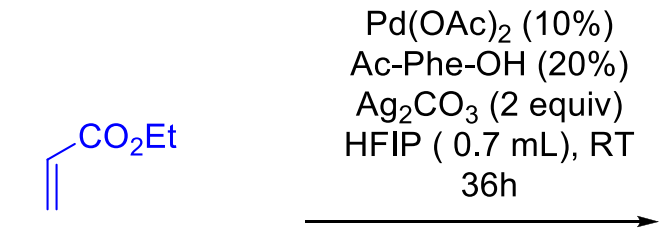

$0.2 \mathrm{mmol}$<smiles>CCOC(=O)C=Cc1cccc(CP(=O)(OCC)Oc2ccc(OC)cc2C#N)c1</smiles>

72\%, 20:1 (m:others)

Scheme S7: Results with MBM scaffolds

\section{Optimization of Hydroxylation:}

\section{Screening of $-\mathrm{OH}$ source:}<smiles>CCOP(=O)(Cc1ccccc1)Oc1ccccc1C#N</smiles>

$0.1 \mathrm{mmol}$

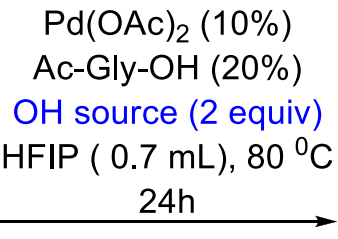<smiles>CCOP(=O)(Cc1cccc(O)c1)Oc1ccccc1C#N</smiles>

Scheme S8: Hydroxyl source screening

Table S7:

\begin{tabular}{|c|c|c|c|c|}
\hline entry & Pd-salt & $-\mathrm{OH}$ source & Yield & $\begin{array}{c}\text { Selectivity } \\
\text { meta:others }\end{array}$ \\
\hline 1 & $\mathrm{Pd}(\mathrm{OAc})_{2}$ & $\mathrm{TBHP}$ & 0 & \\
\hline 2 & $\mathrm{Pd}(\mathrm{OAc})_{2}$ & $\mathrm{H}_{2} \mathrm{O}_{2}$ & 0 & \\
\hline 3 & $\mathrm{Pd}(\mathrm{OAc})_{2}$ & $\mathrm{TEMPO}$ & 0 & \\
\hline
\end{tabular}




\begin{tabular}{|c|c|c|c|c|}
\hline 4 & $\mathrm{Pd}(\mathrm{OAc})_{2}$ & $\mathrm{~K}_{2} \mathrm{~S}_{2} \mathrm{O}_{8}, \mathrm{CF}_{3} \mathrm{COOH}$ & 0 & \\
\hline 5 & $\mathrm{Pd}(\mathrm{OAc})_{2}$ & $\mathrm{Na}_{2} \mathrm{~S}_{2} \mathrm{O}_{8}$, dioxane & 0 & \\
\hline 6 & $\mathrm{Pd}(\mathrm{OAc})_{2}$ & $\mathrm{PhI}(\mathrm{TFA})_{2}, \mathrm{CF}_{3} \mathrm{CH}_{2} \mathrm{OH}$ & 35 & $>20: 1$ \\
\hline 7 & $\mathrm{Pd}(\mathrm{OAc})_{2}$ & $\mathrm{PhI}(\mathrm{TFA})_{2}, \mathrm{HFIP}$ & 62 & $>20: 1$ \\
\hline
\end{tabular}

Yield and selectivity were determined based on ${ }^{1} \mathrm{H}$ NMR of crude reaction mixture using 1,3,5-trimethoxybenzene as an internal standard.

\section{Optimization of PhI(TFA) $)_{2}$ loading:}<smiles>CCOP(=O)(Cc1ccccc1)Oc1ccccc1C#N</smiles>

$0.1 \mathrm{mmol}$
$\mathrm{Pd}(\mathrm{OAc})_{2}(10 \%)$

Ac-Gly-OH (20\%)

$\operatorname{Phl}(\mathrm{TFA})_{2}$ (x equiv)

$\operatorname{HFIP}(0.7 \mathrm{~mL}), 80{ }^{\circ} \mathrm{C}$

$24 \mathrm{~h}$

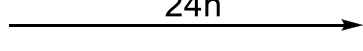<smiles>CCOP(=O)(Cc1cccc(O)c1)Oc1ccccc1C#N</smiles>

Scheme S9: Screening of $\mathrm{PhI}(\mathrm{TFA})_{2}$ loading

Table S8:

\begin{tabular}{|c|c|c|}
\hline entry & PhI(TFA) (equiv) $^{\text {(equeld }(\%)}$ \\
\hline 1 & 2 & 62 \\
\hline 2 & 2.5 & 62 \\
\hline 3 & 3 & 65 \\
\hline 4 & 3.5 & 66 \\
\hline 5 & 4 & 67 \\
\hline
\end{tabular}

Yield and selectivity were determined based on ${ }^{1} \mathrm{H}$ NMR of crude reaction mixture using 1,3,5-trimethoxybenzene as an internal standard.

\section{Ligand Screening:}<smiles>CCOP(=O)(Cc1ccccc1)Oc1ccccc1C#N</smiles>

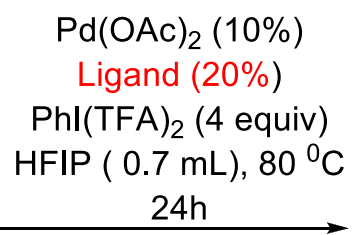

$0.1 \mathrm{mmol}$<smiles>CCOP(=O)(Cc1cccc(O)c1)Oc1ccccc1C#N</smiles>

Scheme S10: Ligand screening

\section{Table S9:}

\begin{tabular}{|c|c|c|}
\hline entry & Ligand & Yield (\%) \\
\hline 1 & Ac-Gly-OH & 67 \\
\hline 2 & Ac-Phe-OH & 64 \\
\hline 3 & For-Gly-OH & 69 \\
\hline 4 & Boc-Gly-OH & 68 \\
\hline 5 & Boc-Val-OH & 69 \\
\hline
\end{tabular}




\begin{tabular}{|c|c|c|}
\hline 6 & Boc-Ala-OH & 74 \\
\hline 7 & Boc-Phe-OH & 70 \\
\hline
\end{tabular}

Yield and selectivity were determined based on ${ }^{1} \mathrm{H}$ NMR of crude reaction mixture using 1,3,5-trimethoxybenzene as an internal standard.

\section{Optimization of Acetoxylation:}

\section{Ligand Screening:}<smiles>CCOP(=O)(Cc1ccccc1)Oc1ccccc1C#N</smiles>

$$
\begin{gathered}
\mathrm{Pd}(\mathrm{OAc})_{2}(10 \%) \\
\text { Ligand }(20 \%) \\
\mathrm{PhI}(\mathrm{OAc})_{2}(4 \text { equiv }) \\
\operatorname{HFIP}(0.7 \mathrm{~mL}), 80^{\circ} \mathrm{C} \\
24 \mathrm{~h} \\
\hline
\end{gathered}
$$

$0.1 \mathrm{mmol}$<smiles>CCOP(=O)(Cc1cccc(OC(C)=O)c1)Oc1ccccc1C#N</smiles>

Scheme S11: Ligand screening

Table S10:

\begin{tabular}{|c|c|c|c|}
\hline entry & Ligand & Yield (\%) & Selectivity (meta:other) \\
\hline 1 & Ac-Gly-OH & 71 & $>20: 1$ \\
\hline 2 & Ac-Phe-OH & 67 &, \\
\hline 3 & For-Gly-OH & 61 &, \\
\hline 4 & Boc-Gly-OH & 58 &, \\
\hline 5 & Boc-Val-OH & 63 &, \\
\hline 6 & Boc-Ala-OH & 68 &, \\
\hline 7 & Boc-Phe-OH & 61 &, \\
\hline
\end{tabular}

Yield and selectivity were determined based on ${ }^{1} \mathrm{H}$ NMR of crude reaction mixture using 1,3,5-trimethoxybenzene as an internal standard.

\section{Optimization with combination of $\mathrm{PhI}(\mathrm{OAc})_{2}$ and $\mathrm{Ac}_{2} \mathrm{O}$ :}<smiles>CCOP(=O)(Cc1ccccc1)Oc1ccccc1C#N</smiles>

$0.1 \mathrm{mmol}$

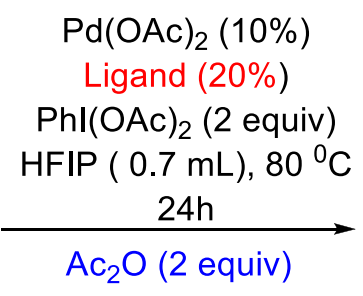<smiles>CCOP(=O)(Cc1cccc(OC(C)=O)c1)Oc1ccccc1C#N</smiles>

Scheme S12: Ligand Screening with $\mathrm{Ac}_{2} \mathrm{O}$

Table S11:

\begin{tabular}{|c|c|c|c|}
\hline entry & Ligand & Yield (\%) & Selectivity (meta: other) \\
\hline 1 & Ac-Gly-OH & 78 & $>20: 1$ \\
\hline 2 & Ac-Phe-OH & 72 &, \\
\hline 3 & For-Gly-OH & 62 &, \\
\hline
\end{tabular}




\begin{tabular}{|c|c|c|c|}
\hline 4 & Boc-Gly-OH & 57 &, \\
\hline 5 & Boc-Val-OH & 66 &, \\
\hline 6 & Boc-Ala-OH & 67 &, \\
\hline 7 & Boc-Phe-OH & 64 &, \\
\hline
\end{tabular}

Yield and selectivity were determined based on ${ }^{1} \mathrm{H}$ NMR of crude reaction mixture using 1,3,5-trimethoxybenzene as an internal standard.

\section{Synthesis of starting materials:}

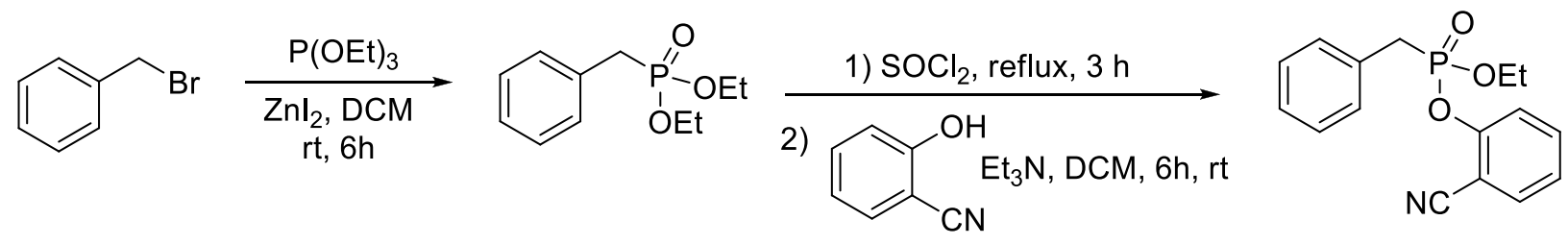

\section{Scheme S13: Starting material synthesis}

Oven dried clean round bottom flask was charged with magnetic stir-bar, benzyl bromide derivative $(1 \mathrm{mmol})$ and $\mathrm{P}(\mathrm{OEt})_{3}(1.2 \mathrm{mmol}) .5 \mathrm{ml}$ of dry $\mathrm{DCM}$ was added to the $\mathrm{RB}$ and stirred at room temperature in the presence of catalytic amount of $\mathrm{ZnI}_{2}(0.2 \mathrm{mmol})$. After $6 \mathrm{~h}$ the reaction was taken out and evaporated under reduced pressure to obtained crude materials which was then purified by column chromatography as petroleum ether/ ethyl acetate as the eluent.

An oven dried clean round bottom flask was charged with magnetic stir-bar and diethyl benzylic phosphonates, and then $\mathrm{SOCl}_{2}$ was added as a solvent amount to the reaction mixture and stirred at reflux condition. After $3 \mathrm{~h}$ the excess amount of $\mathrm{SOCl}_{2}$ was removed by rotavapor and the crude phosphonyl chloride derivatives was charged for next step esterification.

Under nitrogen atmosphere $\mathrm{Et}_{3} \mathrm{~N}$ was added to the 2-hydroxybenzonitrile solution in DCM. The reaction was stirred at $0{ }^{\circ} \mathrm{C}$ for 10 mins. Under nitrogen atmosphere Phosphonyl chloride was added to the reaction in portion until effervescence stopped and then transferred to room temperature and stirred overnight. The progress of the reaction was monitored by TLC. Upon completion the reaction was quenched by adding water and the desired compound was extracted with ethyl acetate. Combined organic portion was dried over anhydrous $\mathrm{Na}_{2} \mathrm{SO}_{4}$. The crude mixture was concentrated under reduced pressure and purified by column chromatography using silica gel (100-200 mesh size) and petroleum ether/ ethyl acetate as the eluent.

\section{General procedure of meta-mono-olefination of Phosphonate ester (Procedure} A):

An oven-dried screw cap reaction tube was charged with a magnetic stir-bar, $\mathrm{Pd}(\mathrm{OAc})_{2}(10$ mol\%, $5 \mathrm{mg}$ ), Ac-Phe-OH (20 mol\%, $8 \mathrm{mg}$ ), $\mathrm{Ag}_{2} \mathrm{CO}_{3}$ (2 equiv.; $110 \mathrm{mg}$ ), corresponding benzylic phosphonate ester $(0.2 \mathrm{mmol})$ and olefin $(0.4 \mathrm{mmol})$. Solid reagents were weighed first followed by liquid reagents. $1.5 \mathrm{~mL}$ of 1,1,1,3,3,3-Hexafluoro-2-propanol (HFIP) were added. The reaction mixture was stirred vigorously at room temperature for $36 \mathrm{~h}$. The reaction was 
taken out after $36 \mathrm{~h}$ and the reaction mixture was diluted with EtOAc and filtered through a celite pad. After filtration and evaporation of the solvent, the crude mixture was purified by column chromatography using silica gel (100-200 mesh size) and petroleum ether/ ethyl acetate as the eluent.

\section{General Procedure of meta-di-olefination of Phosphonate ester (Procedure B):}

An oven-dried screw cap reaction tube was charged with a magnetic stir-bar,, $\mathrm{Pd}(\mathrm{OAc})_{2}(10$ mol\%, $5 \mathrm{mg}$ ), Ac-Gly-OH (20 mol\%, $5 \mathrm{mg}$ ), $\mathrm{Ag}_{2} \mathrm{CO}_{3}$ (2 equiv.; $110 \mathrm{mg}$ ), phosphonate ester $(0.2 \mathrm{mmol})$ and olefin $(0.4 \mathrm{mmol})$. Solid reagents were weighed first followed by liquid reagents. $1.5 \mathrm{~mL}$ of 1,1,1,3,3,3-Hexafluoro-2-propanol (HFIP) was added. The reaction mixture was stirred vigorously on a preheated oil bath at $80{ }^{\circ} \mathrm{C}$ along. The reaction was taken out after $24 \mathrm{~h}$ and the reaction mixture was diluted with EtOAc and filtered through a celite pad. After filtration and evaporation of the solvent, the crude mixture was purified by column chromatography using silica gel (100-200 mesh size) and petroleum ether/ ethyl acetate as the eluent.

\section{General Procedure of meta-heterodi-olefination of Phosphonate ester (Procedure C):}

Hetero di-olefination is a two step process. In first step mono-olefination can be done by following meta-mono olefination condition at room temperature (procedure A). After isolation of mono olefinated product, this product was subjected for another olefination reaction using other olefin following meta-di-olefination reaction condition at $80{ }^{\circ} \mathrm{C}$ (procedure B).

\section{General procedure of meta-hydroxylation of Phosphonate ester:}

An oven-dried screw cap reaction tube was charged with a magnetic stir-bar, $\mathrm{Pd}(\mathrm{OAc})_{2}(10$ mol\%, $5 \mathrm{mg}$ ), Boc-Ala-OH (20 mol\%, $7.5 \mathrm{mg}$ ), PhI(TFA) 2 (4 equiv.; $340 \mathrm{mg}$ ) and corresponding phosphonate ester $(0.2 \mathrm{mmol})$. Solid reagents were weighed first followed by liquid reagents. $1.5 \mathrm{~mL}$ of 1,1,1,3,3,3-Hexafluoro-2-propanol (HFIP) was added. The reaction mixture was stirred vigorously on a preheated oil bath at $80{ }^{\circ} \mathrm{C}$ along. The reaction was taken out after $24 \mathrm{~h}$ and the reaction mixture was diluted with EtOAc and filtered through a celite pad. After filtration and evaporation of the solvent, the crude mixture was purified by column chromatography using silica gel (100-200 mesh size) and petroleum ether/ ethyl acetate as the eluent.

\section{General procedure of meta-acetoxylation of Phosphonate ester:}

An oven-dried screw cap reaction tube was charged with a magnetic stir-bar,, $\operatorname{Pd}(\mathrm{OAc})_{2}(10$ mol\%, 5 mg ), Ac-Gly-OH (20 mol\%, 5 mg), PhI(OAc) $)_{2}$ (2 equiv.; $130 \mathrm{mg}$ ), $\mathrm{Ac}_{2} \mathrm{O}$ (2 equiv, 30 $\mu \mathrm{l})$ and corresponding phosphonate ester $(0.2 \mathrm{mmol})$. Solid reagents were weighed first followed by liquid reagents. $1.5 \mathrm{~mL}$ of 1,1,1,3,3,3-Hexafluoro-2-propanol (HFIP) was added. The reaction mixture was stirred vigorously on a preheated oil bath at $80{ }^{\circ} \mathrm{C}$ along. The reaction 
was taken out after $24 \mathrm{~h}$ and the reaction mixture was diluted with EtOAc and filtered through a celite pad. After filtration and evaporation of the solvent, the crude mixture was purified by column chromatography using silica gel (100-200 mesh size) and petroleum ether/ ethyl acetate as the eluent.

\section{Characterization of the Benzylic Phosphonate ester substrates:}<smiles>CCOP(=O)(Cc1ccccc1)Oc1ccccc1C#N</smiles>

2-cyanophenyl ethyl benzylphosphonate (1a):

${ }^{1}$ H NMR (500 MHz, Chloroform-d) $\delta: 7.59$ (dt, J = 7.7, $\left.1.2 \mathrm{~Hz}, 1 \mathrm{H}\right), 7.47$ (ddd, J = 8.9, 7.5, 1.7 $\mathrm{Hz}, 1 \mathrm{H}), 7.39-7.34(\mathrm{~m}, 2 \mathrm{H}), 7.34-7.31(\mathrm{~m}, 1 \mathrm{H}), 7.31-7.24(\mathrm{~m}, 3 \mathrm{H}), 7.22-7.17(\mathrm{~m}, 1 \mathrm{H})$, $4.23-4.14(\mathrm{~m}, 2 \mathrm{H}), 3.44(\mathrm{~d}, \mathrm{~J}=21.9 \mathrm{~Hz}, 2 \mathrm{H}), 1.25(\mathrm{t}, \mathrm{J}=7.0 \mathrm{~Hz}, 3 \mathrm{H})$.

${ }^{13} \mathrm{C}$ NMR (126 MHz, $\left.\mathrm{CDCl}_{3}\right) \delta$ : 152.31, 134.52, 134.15, 133.62, 133.13, 130.16, 130.11, $128.93,128.90,127.60,127.57,125.24,125.19,121.20,121.18,116.41,115.54,105.67,64.22$, $64.16,34.57,33.48,16.30,16.25$.<smiles>CCOP(=O)(Cc1ccccc1)Oc1ccc(OC)cc1C#N</smiles>

\section{2-cyano-4-methoxyphenyl ethyl benzylphosphonate (1a'):}

${ }^{1}$ H NMR (400 MHz, Chloroform-d) $\delta: ~ 7.34-7.27$ (m, 3H), 7.26 (s, 1H), 7.20 (td, J = 6.8, 1.7 $\mathrm{Hz}, 1 \mathrm{H}), 7.10(\mathrm{dd}, \mathrm{J}=9.1,1.2 \mathrm{~Hz}, 1 \mathrm{H}), 6.99(\mathrm{~d}, \mathrm{~J}=3.2 \mathrm{~Hz}, 1 \mathrm{H}), 6.94(\mathrm{dd}, \mathrm{J}=9.1,3.1 \mathrm{~Hz}, 1 \mathrm{H})$, $4.16-4.05(\mathrm{~m}, 2 \mathrm{H}), 3.75-3.68(\mathrm{~m}, 3 \mathrm{H}), 3.37(\mathrm{~d}, \mathrm{~J}=21.8 \mathrm{~Hz}, 2 \mathrm{H}), 1.18(\mathrm{t}, \mathrm{J}=7.1 \mathrm{~Hz}, 3 \mathrm{H})$.

${ }^{13} \mathrm{C}$ NMR (101 MHz, $\left.\mathrm{CDCl}_{3}\right) \delta: 155.98,145.83,145.75,130.00,129.95,129.91,129.88$, $128.64,128.61,127.24,127.20,122.30,122.27,120.41,117.05,115.30,105.93,63.68,63.60$, $55.81,34.49,33.11,16.14,16.08$.<smiles>CCOP(=O)(Cc1ccc(C)cc1)Oc1ccccc1C#N</smiles> 


\section{2-cyanophenyl ethyl 4-methylbenzylphosphonate (1b):}

${ }^{1}$ H NMR (500 MHz, Chloroform-d) $\delta: 7.58(\mathrm{dd}, \mathrm{J}=7.8,1.6 \mathrm{~Hz}, 1 \mathrm{H}), 7.46(\mathrm{td}, \mathrm{J}=8.0,1.7 \mathrm{~Hz}$, 1H), 7.29 (d, J = 8.5 Hz, 1H), $7.24(\mathrm{dd}, \mathrm{J}=8.1,2.7 \mathrm{~Hz}, 2 \mathrm{H}), 7.18(\mathrm{t}, \mathrm{J}=7.6 \mathrm{~Hz}, 1 \mathrm{H}), 7.11(\mathrm{~d}, \mathrm{~J}=$ $7.7 \mathrm{~Hz}, 2 \mathrm{H}), 4.19-4.12(\mathrm{~m}, 2 \mathrm{H}), 3.39$ (d, J = 21.6 Hz, 2H), 2.30 (d, J = 2.5 Hz, 3H), 1.24 (t, J = $7.0 \mathrm{~Hz}, 3 \mathrm{H})$.

${ }^{13} \mathrm{C}$ NMR (126 MHz, $\left.\mathrm{CDCl}_{3}\right) \delta: 152.61,152.54,137.16,137.12,134.38,133.55,130.00$, $129.95,129.57,129.54,126.80,126.72,124.91,121.23,121.21,115.63,105.64,63.85,63.79$, $34.26,33.15,21.18,16.35,16.30$.<smiles>CCOP(=O)(Cc1ccc(OC)cc1)Oc1ccccc1C#N</smiles>

\section{2-cyanophenyl ethyl 4-methoxybenzylphosphonate (1c):}

${ }^{1}$ H NMR (400 MHz, Chloroform-d) $\delta: 7.62-7.55$ (m, 1H), 7.46 (ddt, J = 9.9, 7.8, $2.0 \mathrm{~Hz}, 1 \mathrm{H}$ ), $7.29-7.21(\mathrm{~m}, 3 \mathrm{H}), 7.21-7.14(\mathrm{~m}, 1 \mathrm{H}), 6.84(\mathrm{dd}, \mathrm{J}=8.4,6.1 \mathrm{~Hz}, 2 \mathrm{H}), 4.20-4.09(\mathrm{~m}, 2 \mathrm{H})$, $3.76(\mathrm{~s}, 3 \mathrm{H}), 3.36(\mathrm{~d}, \mathrm{~J}=21.3 \mathrm{~Hz}, 2 \mathrm{H}), 1.24(\mathrm{t}, \mathrm{J}=7.1 \mathrm{~Hz}, 3 \mathrm{H})$.

${ }^{13} \mathbf{C}$ NMR (101 MHz, $\left.\mathrm{CDCl}_{3}\right) \delta: 159.03,152.58,134.39,133.83,133.54,131.17,131.10$, $125.67,124.93,121.73,121.63,121.19,121.17,115.60,114.33,114.30,105.59,77.52,77.20$, $76.88,63.86,63.78,55.34,33.85,32.47,16.35,16.29$.<smiles>CCOP(=O)(Cc1ccc(Br)cc1)Oc1ccccc1C#N</smiles>

\section{2-cyanophenyl ethyl 4-bromobenzylphosphonate (1d):}

${ }^{1}$ H NMR (500 MHz, Chloroform-d) $\delta: ~ 7.62-7.55(\mathrm{~m}, 1 \mathrm{H}), 7.53-7.47(\mathrm{~m}, 1 \mathrm{H}), 7.42$ (dd, J = 8.3, $3.4 \mathrm{~Hz}, 2 \mathrm{H}), 7.36-7.30(\mathrm{~m}, 1 \mathrm{H}), 7.22(\mathrm{dp}, \mathrm{J}=8.4,3.0 \mathrm{~Hz}, 3 \mathrm{H}), 4.16$ (ddt, J = 8.1, 6.6, 3.2 $\mathrm{Hz}, 2 \mathrm{H}), 3.39$ (d, J = 22.0 Hz, 2H), 1.25 (t, J = 7.1 Hz, 3H).

${ }^{13} \mathrm{C}$ NMR (126 MHz, $\left.\mathrm{CDCl}_{3}\right) \delta: 152.26,152.20,134.54,133.93,133.65,132.00,131.98$, $131.81,131.75,129.05,128.98,125.24,121.67,121.63,121.17,115.53,105.68,64.20,64.14$, $34.13,33.02,16.33,16.29$. 
<smiles>CCOP(=O)(Cc1ccccc1Br)Oc1ccccc1C#N</smiles>

\section{2-cyanophenyl ethyl 2-bromobenzylphosphonate (1e):}

${ }^{1}$ H NMR (500 MHz, Chloroform-d) $\delta: 7.62-7.51(\mathrm{~m}, 3 \mathrm{H}), 7.50-7.45(\mathrm{~m}, 1 \mathrm{H}), 7.35$ (d, J = 8.5 $\mathrm{Hz}, 1 \mathrm{H}), 7.29(\mathrm{dd}, \mathrm{J}=8.7,6.6 \mathrm{~Hz}, 1 \mathrm{H}), 7.20(\mathrm{t}, \mathrm{J}=7.6 \mathrm{~Hz}, 1 \mathrm{H}), 7.12(\mathrm{td}, \mathrm{J}=7.8,2.2 \mathrm{~Hz}, 1 \mathrm{H})$, $4.27-4.12(\mathrm{~m}, 2 \mathrm{H}), 3.69(\mathrm{~d}, \mathrm{~J}=22.3 \mathrm{~Hz}, 2 \mathrm{H}), 1.29-1.22(\mathrm{~m}, 3 \mathrm{H})$.

${ }^{13} \mathrm{C}$ NMR (126 MHz, $\left.\mathrm{CDCl}_{3}\right) \delta: 152.30,152.23,134.32,133.60,133.18,133.16,131.98$, $131.94,130.39,130.32,129.10,129.07,127.78,124.97,120.92,115.43,105.61,64.05,63.99$, $34.48,33.37,16.27,16.22$.<smiles>CCOP(=O)(Cc1cc(F)ccc1F)Oc1ccccc1C#N</smiles>

\section{2-cyanophenyl ethyl 2,5-difluorobenzylphosphonate (1f):}

${ }^{1}$ H NMR (500 MHz, Chloroform-d) $\delta: 7.65-7.61(\mathrm{~m}, 1 \mathrm{H}), 7.57-7.54(\mathrm{~m}, 1 \mathrm{H}), 7.44(\mathrm{dt}, \mathrm{J}=$ 8.5, 1.2 Hz, 1H), $7.27-7.23(\mathrm{~m}, 1 \mathrm{H}), 7.18$ (ddq, J = 8.6, 5.8, 2.7 Hz, 1H), 7.03 (tdd, J = 8.9, 4.6, $1.1 \mathrm{~Hz}, 1 \mathrm{H}), 6.99-6.94(\mathrm{~m}, 1 \mathrm{H}), 4.27-4.21(\mathrm{~m}, 2 \mathrm{H}), 3.49(\mathrm{~d}, \mathrm{~J}=22.0 \mathrm{~Hz}, 2 \mathrm{H}), 1.28(\mathrm{t}, \mathrm{J}=7.0$ $\mathrm{Hz}, 3 \mathrm{H})$.

${ }^{13}$ C NMR (126 MHz, $\left.\mathrm{CDCl}_{3}\right) \delta 152.19,152.13,134.59,134.51,133.75,133.68,125.30,121.04$, $118.50,118.42,118.27,118.23,116.88,116.81,116.68,116.61,116.10,116.03,115.88,115.84$, $115.37,105.81,64.28,64.22,27.61,26.48,16.30,16.26$.<smiles>CCOP(=O)(Cc1cccc(Br)c1)Oc1ccccc1C#N</smiles>

\section{2-cyanophenyl ethyl 3-bromobenzylphosphonate (1g):}

${ }^{1}$ H NMR (500 MHz, Chloroform-d) $\delta: 7.59$ (dt, J = 7.7, $\left.1.9 \mathrm{~Hz}, 1 \mathrm{H}\right), 7.49$ (dq, J = 7.3, $1.5 \mathrm{~Hz}$, $2 \mathrm{H}), 7.41-7.33(\mathrm{~m}, 2 \mathrm{H}), 7.33-7.28(\mathrm{~m}, 1 \mathrm{H}), 7.24-7.18(\mathrm{~m}, 2 \mathrm{H}), 4.18(\mathrm{ttd}, \mathrm{J}=8.7,7.1,5.4$ $\mathrm{Hz}, 2 \mathrm{H}), 3.39(\mathrm{dd}, \mathrm{J}=22.0,1.3 \mathrm{~Hz}, 2 \mathrm{H}), 1.26(\mathrm{td}, \mathrm{J}=7.1,2.1 \mathrm{~Hz}, 3 \mathrm{H})$. 
${ }^{13}$ C NMR (126 MHz, CDCl3) $\delta: 152.37,134.49,133.94,133.66,133.03,132.97,132.40$, $132.33,130.70,130.67,130.39,130.37,128.84,128.79,126.08,125.22,122.74,121.18,115.52$, $105.71,64.13,64.07,34.35,33.25,16.35,16.31$.<smiles>CCOP(=O)(Cc1ccc(F)c(Br)c1)Oc1ccccc1C#N</smiles>

\section{2-cyanophenyl ethyl 3-bromo-4-fluorobenzylphosphonate (1h):}

${ }^{1} \mathbf{H}$ NMR (500 MHz, Chloroform-d) $\delta: 7.63(\mathrm{dt}, \mathrm{J}=7.8,1.2 \mathrm{~Hz}, 1 \mathrm{H}), 7.56-7.48(\mathrm{~m}, 2 \mathrm{H}), 7.38$ $(\mathrm{dt}, \mathrm{J}=8.6,1.2 \mathrm{~Hz}, 1 \mathrm{H}), 7.29(\mathrm{dp}, \mathrm{J}=8.2,2.6,1.8 \mathrm{~Hz}, 1 \mathrm{H}), 7.23(\mathrm{t}, \mathrm{J}=7.6 \mathrm{~Hz}, 1 \mathrm{H}), 7.06$ (tt, J = $8.5,1.2 \mathrm{~Hz}, 1 \mathrm{H}), 4.24-4.18(\mathrm{~m}, 2 \mathrm{H}), 3.37(\mathrm{~d}, \mathrm{~J}=21.7 \mathrm{~Hz}, 2 \mathrm{H}), 1.27$ (td, J = 7.0, $1.4 \mathrm{~Hz}, 3 \mathrm{H})$.

${ }^{13}$ C NMR (101 MHz, CDCl3) $\delta: 152.13,134.83,134.76,134.41,133.56,130.65,130.58$, $130.51,127.56,127.52,127.42,125.18,121.08,121.05,116.81,116.78,116.59,116.56,115.38$, $109.29,105.59,64.04,63.96,33.64,32.25,16.25,16.19$.<smiles>CCOP(=O)(Cc1ccccc1C)Oc1ccccc1C#N</smiles>

\section{2-cyanophenyl ethyl 2-methylbenzylphosphonate (1i):}

${ }^{1} \mathbf{H}$ NMR $\left(500 \mathrm{MHz}, \mathrm{CDCl}_{3}\right) \delta 7.61-7.57(\mathrm{~m}, 1 \mathrm{H}), 7.48-7.43(\mathrm{~m}, 1 \mathrm{H}), 7.35-7.30(\mathrm{~m}, 1 \mathrm{H})$, 7.17 (tdd, $J=13.4,9.4,4.3 \mathrm{~Hz}, 5 \mathrm{H}), 4.14$ (dq, $J=14.2,7.1 \mathrm{~Hz}, 2 \mathrm{H}), 3.47$ (d, $J=22.2 \mathrm{~Hz}, 2 \mathrm{H}$ ), $2.42(\mathrm{~s}, 3 \mathrm{H}), 1.23(\mathrm{t}, J=7.1 \mathrm{~Hz}, 3 \mathrm{H})$.

${ }^{13}$ C NMR (126 MHz, $\left.\mathrm{CDCl}_{3}\right) \delta 152.56,152.51,137.45,137.39,134.42,133.58,131.04,130.99$, $130.82,130.79,128.52,128.44,127.74,127.71,126.41,126.38,124.98,121.19,121.17,115.63$, $105.65,63.88,63.82,32.02,30.91,20.10,16.32,16.27$.<smiles>CCOP(=O)(Cc1cccc(OC)c1)Oc1ccccc1C#N</smiles>

\section{2-cyanophenyl ethyl 3-methylbenzylphosphonate (1j):}

${ }^{1} \mathbf{H}$ NMR $\left(400 \mathrm{MHz}, \mathrm{CDCl}_{3}\right) \delta 7.61-7.56(\mathrm{~m}, 1 \mathrm{H}), 7.49-7.44(\mathrm{~m}, 1 \mathrm{H}), 7.29(\mathrm{~d}, J=8.5 \mathrm{~Hz}$, $1 \mathrm{H}), 7.25-7.18(\mathrm{~m}, 2 \mathrm{H}), 7.18-7.12(\mathrm{~m}, 2 \mathrm{H}), 7.06(\mathrm{~d}, J=7.8 \mathrm{~Hz}, 1 \mathrm{H}), 4.23-4.13(\mathrm{~m}, 2 \mathrm{H})$, $3.39(\mathrm{~d}, J=21.8 \mathrm{~Hz}, 2 \mathrm{H}), 2.31(\mathrm{~s}, 3 \mathrm{H}), 1.25(\mathrm{t}, J=7.1 \mathrm{~Hz}, 3 \mathrm{H})$. 
${ }^{13}$ C NMR (101 MHz, $\left.\mathrm{CDCl}_{3}\right) \delta 152.60,152.52,138.57,138.54,134.39,133.84,133.54,130.90$, $130.84,129.78,129.69,128.76,128.72,128.26,128.22,127.18,127.11,125.69,124.94,121.17$, $121.14,115.61,105.60,63.89,63.82,34.74,33.36,21.39,16.34,16.28$.<smiles>CCOP(=O)(Cc1ccc(C(C)(C)C)cc1)Oc1ccccc1C#N</smiles>

2-cyanophenyl ethyl 4-tert-butylbenzylphosphonate (1k):

${ }^{1} \mathbf{H}$ NMR $\left(400 \mathrm{MHz}, \mathrm{CDCl}_{3}\right) \delta 7.55(\mathrm{~d}, J=7.9 \mathrm{~Hz}, 1 \mathrm{H}), 7.45-7.37(\mathrm{~m}, 1 \mathrm{H}), 7.34-7.24(\mathrm{~m}$, 4H), $7.21-7.12$ (m, 2H), $4.26-4.11(\mathrm{~m}, 2 \mathrm{H}), 3.40$ (d, $J=21.6 \mathrm{~Hz}, 2 \mathrm{H}), 1.28$ (s, 9H), 1.25 (d, $J$ $=7.0 \mathrm{~Hz}, 3 \mathrm{H})$.

${ }^{13}$ C NMR (126 MHz, $\left.\mathrm{CDCl}_{3}\right) \delta 152.60,152.54,150.43,150.40,134.27,133.45,129.81,129.76$, $126.68,126.61,125.79,125.76,124.85,121.10,121.08,115.57,105.57,63.79,63.73,34.12$, $33.01,31.35,16.29,16.24$.

\section{Characterization of the meta-olefinated compounds:}

\section{Meta-selective mono-olefination}<smiles>CCOC(=O)Cc1cccc(CP(=O)(OCC)Oc2ccccc2C)c1</smiles>

(E)-ethyl 3-(3-(((2-cyanophenoxy)(ethoxy)phosphoryl)methyl)phenyl)acrylate (2a):

Yield : 84\% (67 mg)

Appearance: Liquid

$\mathbf{R}_{\mathbf{f}}: 0.3$ (30\% ethyl acetate/pet-ether mixture)

Isolation: Petroleum ether :ethyl acetate(v/v) (13:7)

${ }^{1}$ H NMR (500 MHz, Chloroform-d) $\delta: 7.66(\mathrm{~d}, \mathrm{~J}=16.0 \mathrm{~Hz}, 1 \mathrm{H}), 7.61-7.58(\mathrm{~m}, 1 \mathrm{H}), 7.52-$ $7.48(\mathrm{~m}, 2 \mathrm{H}), 7.43(\mathrm{dd}, \mathrm{J}=7.6,1.9 \mathrm{~Hz}, 1 \mathrm{H}), 7.40(\mathrm{dt}, \mathrm{J}=8.4,1.9 \mathrm{~Hz}, 1 \mathrm{H}), 7.34(\mathrm{dt}, \mathrm{J}=7.7,3.4$ 
$\mathrm{Hz}, 2 \mathrm{H}), 7.21(\mathrm{t}, \mathrm{J}=7.6 \mathrm{~Hz}, 1 \mathrm{H}), 6.45(\mathrm{~d}, \mathrm{~J}=16.0 \mathrm{~Hz}, 1 \mathrm{H}), 4.28(\mathrm{q}, \mathrm{J}=7.1 \mathrm{~Hz}, 2 \mathrm{H}), 4.16$ (ddq, J $=12.3,7.0,3.9,3.3 \mathrm{~Hz}, 2 \mathrm{H}), 3.47(\mathrm{~d}, \mathrm{~J}=21.9 \mathrm{~Hz}, 2 \mathrm{H}), 1.33(\mathrm{t}, \mathrm{J}=7.1 \mathrm{~Hz}, 3 \mathrm{H}), 1.24(\mathrm{t}, \mathrm{J}=7 \mathrm{~Hz}$, $3 \mathrm{H})$.

${ }^{13}$ C NMR (101 MHz, $\left.\mathrm{CDCl}_{3}\right) \delta: 167.04,152.35,144.11,135.23,135.20,134.52,133.69$, $132.00,131.94,129.79,129.72,129.51,127.24,127.20,125.20,121.27,119.12,115.62,105.87$, $64.13,64.06,60.74,34.87,33.48,16.45,14.49$.

HRMS: $\left[(\mathrm{M}+\mathrm{Na})^{+}\right]$Calcd.: 422.1128; observed: 422.1130<smiles>CCOP(=O)(Cc1cccc(/C=C/C(C)=O)c1)Oc1ccccc1C#N</smiles>

(E)-methyl 3-(3-(((2-cyanophenoxy)(ethoxy)phosphoryl)methyl)phenyl)acrylate(2b):

Yield : 82\% (63 mg)

Appearance: Liquid

$\mathbf{R}_{\mathbf{f}}: 0.3$ (30\% ethyl acetate/pet-ether mixture)

Isolation: Petroleum ether :ethyl acetate(v/v) (13:7)

${ }^{1}$ H NMR (400 MHz, Chloroform-d) $\delta 7.64(\mathrm{~d}, \mathrm{~J}=16.1 \mathrm{~Hz}, 2 \mathrm{H}), 7.60(\mathrm{dt}, \mathrm{J}=7.8,1.2 \mathrm{~Hz}, 1 \mathrm{H})$, $7.53(\mathrm{~d}, \mathrm{~J}=2.6 \mathrm{~Hz}, 3 \mathrm{H}), 7.51-7.47(\mathrm{~m}, 1 \mathrm{H}), 7.41-7.37(\mathrm{~m}, 1 \mathrm{H}), 7.24-7.20(\mathrm{~m}, 1 \mathrm{H}), 6.45(\mathrm{~d}$, $\mathrm{J}=16 \mathrm{~Hz}, 1 \mathrm{H}), 4.26-4.14(\mathrm{~m}, 2 \mathrm{H}), 3.80(\mathrm{~s}, 1 \mathrm{H}), 3.46(\mathrm{~d}, \mathrm{~J}=22.0 \mathrm{~Hz}, 2 \mathrm{H}), 1.26(\mathrm{t}, \mathrm{J}=7.1 \mathrm{~Hz}$, $3 \mathrm{H})$.

${ }^{13} \mathrm{C}$ NMR (101 MHz, $\left.\mathrm{CDCl}_{3}\right) \delta: 167.20,152.31,143.51,135.82,135.79,134.55,133.69$, $132.04,131.94,131.07,131.00,126.88,126.85,125.32,121.22,121.20,119.59,115.57,105.77$, $64.19,64.11,52.02,34.74,33.36,16.46$.

HRMS: $\left[(\mathrm{M}+\mathrm{Na})^{+}\right]$Calcd.: 408.0971; observed: 408.0976.<smiles>CCOP(=O)(Cc1cccc(/C=C/C(=O)OCc2ccccc2)c1)Oc1ccccc1C#N</smiles>

(E)-benzyl 3-(3-(((2-cyanophenoxy)(ethoxy)phosphoryl)methyl)phenyl)acrylate (2c):

Yield : 68\% (62 mg)

Appearance: Liquid 
$\mathbf{R}_{\mathbf{f}}: 0.3$ (30\% ethyl acetate/pet-ether mixture)

Isolation: Petroleum ether :ethyl acetate(v/v) (13:7)

${ }^{1}$ H NMR (400 MHz, Chloroform-d) $\delta: 7.68(\mathrm{~d}, \mathrm{~J}=16.0 \mathrm{~Hz}, 1 \mathrm{H}), 7.58(\mathrm{dd}, \mathrm{J}=7.8,1.6 \mathrm{~Hz}, 2 \mathrm{H})$, $7.52-7.46(\mathrm{~m}, 2 \mathrm{H}), 7.41-7.37(\mathrm{~m}, 3 \mathrm{H}), 7.36-7.33(\mathrm{~m}, 3 \mathrm{H}), 7.32-7.27(\mathrm{~m}, 1 \mathrm{H}), 7.19(\mathrm{td}, \mathrm{J}=$ 7.7, $2.5 \mathrm{~Hz}, 2 \mathrm{H}), 6.48(\mathrm{~d}, \mathrm{~J}=16.0 \mathrm{~Hz}, 1 \mathrm{H}), 5.24(\mathrm{~s}, 2 \mathrm{H}), 4.21-4.15(\mathrm{~m}, 2 \mathrm{H}), 3.44(\mathrm{~d}, \mathrm{~J}=22.0$ $\mathrm{Hz}, 2 \mathrm{H}), 1.24$ (t, J = 7.1 Hz, 3H).

${ }^{13} \mathrm{C}$ NMR (101 MHz, $\left.\mathrm{CDCl}_{3}\right) \delta: 166.74,152.36,144.65,136.16,134.46,134.40,133.63$, $133.59,132.09,132.02,131.09,130.21,130.14,129.75,129.47,128.91,128.71,128.36,127.18$, $125.14,124.98,121.24,118.66,115.55,105.73,66.50,64.05,63.97,34.78,33.40,16.40$.

HRMS: $\left[(\mathrm{M}+\mathrm{Na})^{+}\right]$Calcd.: 484.1284; observed: 484.1278 .<smiles>CCOP(=O)(Cc1cccc(/C=C/C(C)=O)c1)Oc1ccccc1C#N</smiles>

\section{(E)-2-cyanophenyl ethyl 3-(3-oxobut-1-en-1-yl)benzylphosphonate (2d):}

Yield : 80\% (59 mg)

Appearance: Liquid

$\mathbf{R}_{\mathbf{f}}: 0.4$ (50\% ethyl acetate/pet-ether mixture)

Isolation: Petroleum ether :ethyl acetate $(\mathrm{v} / \mathrm{v})(3: 2)$

${ }^{1}$ H NMR (500 MHz, Chloroform-d) $\delta: 7.59(\mathrm{dt}, \mathrm{J}=7.7,1.2 \mathrm{~Hz}, 1 \mathrm{H}), 7.55$ (dd, J = 3.0, $1.6 \mathrm{~Hz}$, $1 \mathrm{H}), 7.52-7.43(\mathrm{~m}, 3 \mathrm{H}), 7.42-7.33(\mathrm{~m}, 3 \mathrm{H}), 7.21(\mathrm{tt}, \mathrm{J}=7.6,0.9 \mathrm{~Hz}, 1 \mathrm{H}), 6.71(\mathrm{~d}, \mathrm{~J}=16.3$ $\mathrm{Hz}, 1 \mathrm{H}), 4.18(\mathrm{dqd}, \mathrm{J}=8.3,7.0,5.5 \mathrm{~Hz}, 2 \mathrm{H}), 3.45(\mathrm{~d}, \mathrm{~J}=21.9 \mathrm{~Hz}, 2 \mathrm{H}), 2.36(\mathrm{~s}, 3 \mathrm{H}), 1.25(\mathrm{t}, \mathrm{J}=$ $7.0 \mathrm{~Hz}, 3 \mathrm{H})$.

${ }^{13} \mathrm{C}$ NMR (126 MHz, $\left.\mathrm{CDCl}_{3}\right) \delta: 198.50,152.45,142.96,135.21,135.18,134.51,133.69$, $132.22,132.17,131.17,131.09,130.07,130.02,129.58,129.56,127.76,127.33,127.30,125.18$, $121.20,121.18,115.62,105.71,64.11,64.05,34.68,33.58,27.76,16.41,16.37$.

HRMS: $\left[(\mathrm{M}+\mathrm{Na})^{+}\right]$Calcd.: 392.1022; observed: 392.1027 .

IR (thin film): 2983, 2232, 1669, 1601, 1488, 1261, 1037, 921, $694 \mathrm{~cm}^{-1}$<smiles>CCOP(=O)(/C=C/c1cccc(CP(=O)(OCC)OCC)c1)OCC</smiles> 


\section{(E)-2-cyanophenyl ethyl 3-(2-(diethoxyphosphoryl)vinyl)benzylphosphonate (2e):}

Yield : 65\% (60 mg)

Appearance: Liquid

$\mathbf{R}_{\mathbf{f}}: 0.2$ (80\% ethyl acetate/pet-ether mixture)

Isolation: Ethyl acetate :Methanol (v/v) (20:1)

${ }^{1}$ H NMR (500 MHz, Chloroform-d) $\delta: 7.59$ (dd, J = 7.8, $\left.1.6 \mathrm{~Hz}, 1 \mathrm{H}\right), 7.52-7.43$ (m, 3H), 7.42 - $7.37(\mathrm{~m}, 2 \mathrm{H}), 7.34(\mathrm{~d}, \mathrm{~J}=8.0 \mathrm{~Hz}, 2 \mathrm{H}), 7.23-7.18(\mathrm{~m}, 1 \mathrm{H}), 6.26(\mathrm{t}, \mathrm{J}=17.5 \mathrm{~Hz}, 1 \mathrm{H}), 4.15$ (dddd, J = 28.6, 10.2, 7.3, 3.8 Hz, 6H), $3.44(\mathrm{~d}, \mathrm{~J}=21.9 \mathrm{~Hz}, 2 \mathrm{H}), 1.34(\mathrm{t}, \mathrm{J}=7.1 \mathrm{~Hz}, 6 \mathrm{H}), 1.25$ (t, J $=7.0 \mathrm{~Hz}, 3 \mathrm{H})$.

${ }^{13} \mathbf{C}$ NMR (126 MHz, $\left.\mathrm{CDCl}_{3}\right) \delta: 152.52,152.45,148.20,148.15,135.67,135.49,134.50$, 133.66, 131.98, 131.93, 131.03, 130.95, 129.48, 129.46, 129.27, 129.22, 127.05, 127.03, 125.17, $121.22,121.20,115.63,115.60,114.11,105.72,64.05,63.99,62.09,62.05,34.68,33.58,16.59$, $16.54,16.41,16.37$.

HRMS: $\left[(\mathrm{M}+\mathrm{Na})^{+}\right]$Calcd.: 486.1180; observed: 486.1180 .<smiles>CCOP(=O)(Cc1cccc(/C=C/SOC(C)(F)F)c1)Oc1ccccc1C#N</smiles>

\section{(E)-2-cyanophenyl ethyl 3-(2-(methylsulfonyl)vinyl)benzylphosphonate (2f):}

Yield : 78\% (63 mg)

Appearance: Liquid

$\mathbf{R}_{\mathbf{f}}: 0.3$ (50\% ethyl acetate/pet-ether mixture)

Isolation: Petroleum ether :ethyl acetate $(\mathrm{v} / \mathrm{v})(1: 1)$

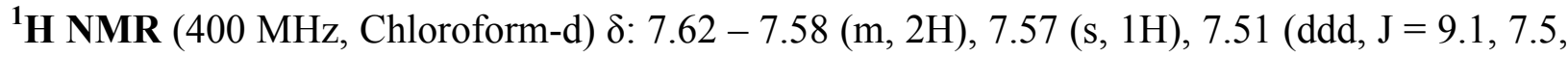
$1.7 \mathrm{~Hz}, 1 \mathrm{H}), 7.45$ (dq, J = 4.6, 2.3 Hz, 1H), 7.40 (t, J = 4.6 Hz, 3H), 7.22 (t, J = 7.6 Hz, 1H), 6.96 $(\mathrm{d}, \mathrm{J}=15.5 \mathrm{~Hz}, 1 \mathrm{H}), 4.27-4.12(\mathrm{~m}, 2 \mathrm{H}), 3.46(\mathrm{~d}, \mathrm{~J}=22.0 \mathrm{~Hz}, 2 \mathrm{H}), 3.03(\mathrm{~s}, 3 \mathrm{H}), 1.27(\mathrm{t}, \mathrm{J}=7.0$ $\mathrm{Hz}, 3 \mathrm{H})$.

${ }^{13} \mathbf{C}$ NMR (101 MHz, $\left.\mathrm{CDCl}_{3}\right) \delta: 152.40,143.40,134.59,133.75,133.10,133.04,132.88$, $131.57,129.88,129.80,129.76,128.16,128.12,127.17,125.26,121.15,121.13,115.65,105.70$, $64.20,64.12,43.42,34.86,33.47,16.47,16.41$.

HRMS: $\left[(\mathrm{M}+\mathrm{Na})^{+}\right]$Calcd.: 428.0692; observed: 428.0698 
<smiles>CCOP(=O)(Cc1cccc(/C=C/S(=O)(=O)c2ccccc2)c1)Oc1ccccc1C</smiles>

(E)-2-cyanophenyl ethyl 3-(2-(phenylsulfonyl)vinyl)benzylphosphonate (2g):

Yield : 75\% (69.5 mg)

Appearance: Liquid

$\mathbf{R}_{\mathbf{f}}: 0.3$ ( $50 \%$ ethyl acetate/pet-ether mixture)

Isolation: Petroleum ether :ethyl acetate (v/v) (1:1)

${ }^{1}$ H NMR (400 MHz, Chloroform-d) $\delta: 7.99-7.91(\mathrm{~m}, 2 \mathrm{H}), 7.68-7.60(\mathrm{~m}, 2 \mathrm{H}), 7.58-7.53(\mathrm{~m}$, $3 \mathrm{H}), 7.51-7.46(\mathrm{~m}, 2 \mathrm{H}), 7.42(\mathrm{dq}, \mathrm{J}=6.4,2.2 \mathrm{~Hz}, 1 \mathrm{H}), 7.39-7.34(\mathrm{~m}, 3 \mathrm{H}), 7.19$ (tt, J = 7.7, $0.9 \mathrm{~Hz}, 1 \mathrm{H}), 6.89(\mathrm{~d}, \mathrm{~J}=15.4 \mathrm{~Hz}, 1 \mathrm{H}), 4.17$ (ddtd, $\mathrm{J}=10.8,9.0,7.1,3.9 \mathrm{~Hz}, 2 \mathrm{H}), 3.43(\mathrm{~d}, \mathrm{~J}=$ $22.0 \mathrm{~Hz}, 2 \mathrm{H}), 1.24(\mathrm{t}, \mathrm{J}=7.1 \mathrm{~Hz}, 3 \mathrm{H})$.

${ }^{13}$ C NMR $\left(101 \mathrm{MHz}, \mathrm{CDCl}_{3}\right) \delta: 152.41,141.93,140.77,134.53,133.68,133.59,133.15$, $133.11,132.95,132.88,131.45,131.35,129.98,129.91,129.72,129.69,129.51,128.19,128.02$, $127.99,127.91,125.23,121.17,121.14,115.58,105.67,64.15,64.07,34.78,33.39,16.42$, 16.36 .

HRMS: $\left[(\mathrm{M}+\mathrm{Na})^{+}\right]$Calcd.: 490.0849; observed: 490.0844 .<smiles>C=COS(=O)(=O)Cc1cccc(/C=C/S(=O)(=O)Oc2ccccc2C#N)c1</smiles>

(E)-2-cyanophenyl ethyl 3-(2-(vinylsulfonyl)vinyl)benzylphosphonate (2h):

Yield : $82 \%(68 \mathrm{mg})$

Appearance: Liquid

$\mathbf{R}_{\mathbf{f}}: 0.3$ (50\% ethyl acetate/pet-ether mixture)

Isolation: Petroleum ether :ethyl acetate (v/v) (1:1)

${ }^{1}$ H NMR (400 MHz, Chloroform-d) $\delta: 7.60(\mathrm{~d}, \mathrm{~J}=7.3 \mathrm{~Hz}, 1 \mathrm{H}), 7.56-7.48(\mathrm{~m}, 3 \mathrm{H}), 7.45$ (dq, J $=6.2,2.1 \mathrm{~Hz}, 1 \mathrm{H}), 7.38(\mathrm{~d}, \mathrm{~J}=9.3 \mathrm{~Hz}, 3 \mathrm{H}), 7.22(\mathrm{t}, \mathrm{J}=7.6 \mathrm{~Hz}, 1 \mathrm{H}), 6.81(\mathrm{~d}, \mathrm{~J}=15.5 \mathrm{~Hz}, 1 \mathrm{H})$, $6.65(\mathrm{dd}, \mathrm{J}=16.6,9.8 \mathrm{~Hz}, 1 \mathrm{H}), 6.45(\mathrm{~d}, \mathrm{~J}=16.6 \mathrm{~Hz}, 1 \mathrm{H}), 6.09(\mathrm{~d}, \mathrm{~J}=9.8 \mathrm{~Hz}, 1 \mathrm{H}), 4.19(\mathrm{dqd}, \mathrm{J}=$ 8.4, 7.1, 4.9 Hz, 2H), $3.45(\mathrm{~d}, \mathrm{~J}=22.0 \mathrm{~Hz}, 2 \mathrm{H}), 1.25(\mathrm{t}, \mathrm{J}=7.1 \mathrm{~Hz}, 3 \mathrm{H})$. 
${ }^{13}$ C NMR $\left(101 \mathrm{MHz}, \mathrm{CDCl}_{3}\right) \delta: 152.31,143.92,137.81,134.55,133.71,133.12,133.06$, $131.56,131.46,130.01,129.94,129.76,129.73,128.99,128.03,127.99,126.34,125.26,121.15$, $115.59,105.75,64.15,64.07,34.80,33.42,16.43,16.38$.

HRMS: $\left[(\mathrm{M}+\mathrm{Na})^{+}\right]$Calcd.: 440.0692; observed: 440.0692 .<smiles>CCOP(=O)(Cc1cccc(C(=CC(C)=O)C(C)=O)c1)Oc1ccccc1C</smiles>

Dimethyl 2-(3-(((2-cyanophenoxy)(ethoxy)phosphoryl)methyl)phenyl)maleate (2i):

Yield : $71 \%(62 \mathrm{mg})$

Appearance: Liquid

$\mathbf{R}_{\mathbf{f}}: 0.3$ (50\% ethyl acetate/pet-ether mixture)

Isolation: Petroleum ether :ethyl acetate (v/v) (1:1)

${ }^{1}$ H NMR (400 MHz, Chloroform-d) $\delta: 7.59$ (dt, J = 7.7, 1.2 Hz, 1H), 7.49 (ddd, J = 8.5, 7.6, 1.8 $\mathrm{Hz}, 1 \mathrm{H}), 7.45(\mathrm{q}, \mathrm{J}=2.2 \mathrm{~Hz}, 2 \mathrm{H}), 7.39-7.35(\mathrm{~m}, 2 \mathrm{H}), 7.33(\mathrm{dt}, \mathrm{J}=8.4,1.2 \mathrm{~Hz}, 1 \mathrm{H}), 7.21(\mathrm{tt}, \mathrm{J}=$ 7.7, $0.9 \mathrm{~Hz}, 1 \mathrm{H}), 6.31$ (s, 1H), 4.19 (dddd, J = 10.1, 7.0, 3.2, 1.6 Hz, 2H), 3.92 (s, 3H), 3.78 (s, $3 \mathrm{H}), 3.45(\mathrm{~d}, \mathrm{~J}=22.0 \mathrm{~Hz}, 2 \mathrm{H}), 1.27(\mathrm{t}, \mathrm{J}=7.2 \mathrm{~Hz}, 3 \mathrm{H})$.

${ }^{13}$ C NMR (101 MHz, CDCl3) $\delta: 168.28,165.50,152.33,148.47,134.53,133.68,132.44$, $132.38,131.32,129.69,129.66,128.38,128.30,126.11,126.07,125.23,121.24,117.90,115.58$, $105.83,64.14,64.07,52.94,52.25,34.87,33.48,29.85,16.40,16.34$.

HRMS: Calcd.: 466.1035; observed: 466.1038.<smiles>CCOP(=O)(Cc1cccc(C2CCCC=C2OC)c1)Oc1ccccc1C#N</smiles>

2-Cyanophenyl ethyl $\quad\left(\left(6^{\prime}\right.\right.$-acetyl-1',2',3',4'-tetrahydro-[1,1'-biphenyl]-3-
yl)methyl)phosphonate (2j):

Yield : 79\% $(66 \mathrm{mg})$

Appearance: Liquid

$\mathbf{R}_{\mathbf{f}}: 0.3$ (30\% ethyl acetate/pet-ether mixture)

Isolation: Petroleum ether :ethyl acetate (v/v) (3:2) 
${ }^{1}$ H NMR (400 MHz, Chloroform-d) $\delta: 7.56(\mathrm{dt}, \mathrm{J}=7.8,2.5 \mathrm{~Hz}, 1 \mathrm{H}), 7.45(\mathrm{qd}, \mathrm{J}=8.5,1.8 \mathrm{~Hz}$, $1 \mathrm{H}), 7.22-7.12(\mathrm{~m}, 4 \mathrm{H}), 7.09$ (ddd, $\mathrm{J}=5.5,3.7,1.5 \mathrm{~Hz}, 1 \mathrm{H}), 7.07-6.82(\mathrm{~m}, 2 \mathrm{H}), 4.16$ (ttd, $\mathrm{J}=$ 7.0, 3.4, $1.8 \mathrm{~Hz}, 2 \mathrm{H}), 3.99(\mathrm{~d}, \mathrm{~J}=4.7 \mathrm{~Hz}, 1 \mathrm{H}), 3.39(\mathrm{dd}, \mathrm{J}=21.9,4.4 \mathrm{~Hz}, 2 \mathrm{H}), 2.44-2.37(\mathrm{~m}$, 1H), $2.37-2.12(\mathrm{~m}, 4 \mathrm{H}), 1.90-1.63(\mathrm{~m}, 2 \mathrm{H}), 1.55-1.35(\mathrm{~m}, 2 \mathrm{H}), 1.26(\mathrm{t}, \mathrm{J}=7.1 \mathrm{~Hz}, 3 \mathrm{H})$.

${ }^{13}$ C NMR $\left(126 \mathrm{MHz}, \mathrm{CDCl}_{3}\right) \delta: 198.69,152.54,145.97,142.69,140.90,134.63,133.46$, $129.40,129.35,128.92,128.89,127.97,127.91,127.40,127.19,124.96,121.31,120.13,115.75$, $105.58,63.89,63.83,38.43,34.80,33.70,31.30,26.29,26.02,16.95,16.40,16.35$.

HRMS: $\left[(\mathrm{M}+\mathrm{Na})^{+}\right]$Calcd.: 446.1492 ; observed: 446.1494 .

IR (thin film): 2946, 2234, 1662, 1450, 1238, 1029, 925, $767 \mathrm{~cm}^{-1}$<smiles>CCOP(=O)(Cc1cccc(/C=C/c2ccc(OC(C)=O)cc2)c1)Oc1ccccc1C</smiles>

(E)-4-(3-(((2-cyanophenoxy)(ethoxy)phosphoryl)methyl)styryl)phenyl acetate (2k):

Yield : $56 \%(51 \mathrm{mg})$

Appearance: Liquid

$\mathbf{R}_{\mathbf{f}}: 0.4$ (30\% ethyl acetate/pet-ether mixture)

Isolation: Petroleum ether :ethyl acetate (v/v) (3:1)

${ }^{1}$ H NMR (400 MHz, Chloroform-d) $\delta: 7.59$ (dt, J = 7.7, 1.2 Hz, 1H), $7.54-7.44$ (m, 4H), 7.40 $(\mathrm{dq}, \mathrm{J}=7.6,1.8 \mathrm{~Hz}, 1 \mathrm{H}), 7.36-7.28(\mathrm{~m}, 2 \mathrm{H}), 7.26(\mathrm{~s}, 1 \mathrm{H}), 7.19(\mathrm{ddt}, \mathrm{J}=8.5,7.6,0.8 \mathrm{~Hz}, 1 \mathrm{H})$, $7.13-7.05(\mathrm{~m}, 3 \mathrm{H}), 7.01(\mathrm{~d}, \mathrm{~J}=16.4 \mathrm{~Hz}, 1 \mathrm{H}), 4.20(\mathrm{dqd}, \mathrm{J}=10.7,7.1,2.2 \mathrm{~Hz}, 2 \mathrm{H}), 3.46(\mathrm{~d}, \mathrm{~J}=$ $21.9 \mathrm{~Hz}, 2 \mathrm{H}), 2.31(\mathrm{~s}, 3 \mathrm{H}), 1.26(\mathrm{t}, \mathrm{J}=6.9 \mathrm{~Hz}, 3 \mathrm{H})$.

${ }^{13} \mathbf{C}$ NMR $\left(126 \mathrm{MHz}, \mathrm{CDCl}_{3}\right) \delta: 169.62,152.68,150.31,137.89,137.87,135.15,134.48$, $133.63,130.54,130.47,129.49,129.44,129.30,129.27,128.47,128.35,128.28,128.23,127.64$, $125.77,125.74,125.04,121.98,121.19,115.71,105.68,64.05,63.99,34.81,33.71,21.32$, $16.44,16.39$.

HRMS: $\left[(\mathrm{M}+\mathrm{Na})^{+}\right]$Calcd.: 484.1284; observed: 484.1287 .

IR (thin film): 2932, 2233, 1759, 1600, 1230, 1220, 1168, 1034, 919, $827 \mathrm{~cm}^{-1}$ 
<smiles>CCOP(=O)(Cc1cccc(/C=C/c2cccc([N+](=O)[O-])c2)c1)Oc1ccccc1C#N</smiles>

(E)-2-cyanophenyl ethyl 3-(3-nitrostyryl)benzylphosphonate (2l):

Yield : 61\% (55 mg)

Appearance: Liquid

$\mathbf{R}_{\mathbf{f}}: 0.4$ (30\% ethyl acetate/pet-ether mixture)

Isolation: Petroleum ether :ethyl acetate (v/v) (3:1)

${ }^{1}$ H NMR (500 MHz, Chloroform-d) $\delta: 8.41(\mathrm{t}, \mathrm{J}=2.0 \mathrm{~Hz}, 1 \mathrm{H}), 8.11(\mathrm{dd}, \mathrm{J}=8.2,2.2 \mathrm{~Hz}, 1 \mathrm{H})$, $7.79(\mathrm{~d}, \mathrm{~J}=7.8 \mathrm{~Hz}, 1 \mathrm{H}), 7.64-7.56(\mathrm{~m}, 2 \mathrm{H}), 7.56-7.46(\mathrm{~m}, 2 \mathrm{H}), 7.46-7.40(\mathrm{~m}, 1 \mathrm{H}), 7.40-$ 7.28 (m, 3H), $7.24-7.19(\mathrm{~m}, 3 \mathrm{H}), 4.21$ (dtq, J = 14.4, 7.3, 4.7, 3.8 Hz, 2H), 3.48 (d, J = 21.8 Hz, $2 \mathrm{H}), 1.27(\mathrm{t}, \mathrm{J}=7.1 \mathrm{~Hz}, 3 \mathrm{H})$.

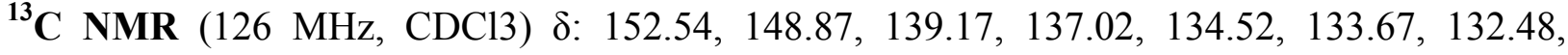
$131.29,130.82,130.75,130.27,130.21,129.76,129.42,128.54,128.48,126.85,126.16,125.11$, $122.28,121.20,121.09,115.70,105.67,64.10,64.04,34.78,33.68,16.45,16.40$.

HRMS: $\left[(\mathrm{M}+\mathrm{Na})^{+}\right]$Calcd.: 471.1080; observed: 471.1076 .

IR (thin film): 2983, 2233, 1600, 1529, 1488, 1351, 1262, 1237, 1034, 919, 807, $697 \mathrm{~cm}^{-1}$<smiles>CCOC(=O)C=Cc1cc(CP(=O)(OCC)Oc2ccccc2C#N)ccc1C</smiles>

(E)-ethyl 3-(5-(((2-cyanophenoxy)(ethoxy)phosphoryl)methyl)-2-methylphenyl)acrylate (2m):

Yield : 74\% (61 mg)

Appearance: Liquid

$\mathbf{R}_{\mathbf{f}}: 0.3$ (30\% ethyl acetate/pet-ether mixture)

Isolation: Petroleum ether :ethyl acetate(v/v) (13:7)

${ }^{1}$ H NMR (400 MHz, Chloroform-d) $\delta: 7.91(\mathrm{~d}, \mathrm{~J}=15.9 \mathrm{~Hz}, 1 \mathrm{H}), 7.59$ (dt, J = 7.7, $\left.1.2 \mathrm{~Hz}, 1 \mathrm{H}\right)$, $7.52-7.45(\mathrm{~m}, 3 \mathrm{H}), 7.35(\mathrm{~d}, \mathrm{~J}=8.5 \mathrm{~Hz}, 1 \mathrm{H}), 7.20(\mathrm{t}, \mathrm{J}=7.6 \mathrm{~Hz}, 1 \mathrm{H}), 7.14(\mathrm{~d}, \mathrm{~J}=7.8 \mathrm{~Hz}, 1 \mathrm{H})$, 
$6.36(\mathrm{~d}, \mathrm{~J}=15.8 \mathrm{~Hz}, 1 \mathrm{H}), 4.26-4.22(\mathrm{~m}, 2 \mathrm{H}), 4.13(\mathrm{dtd}, \mathrm{J}=9.0,7.1,2.2 \mathrm{~Hz}, 2 \mathrm{H}), 3.43(\mathrm{~d}, \mathrm{~J}=$ $21.7 \mathrm{~Hz}, 2 \mathrm{H}), 2.39$ (d, J = 2.3 Hz, 3H), $1.31(\mathrm{t}, \mathrm{J}=7.1 \mathrm{~Hz}, 3 \mathrm{H}), 1.26(\mathrm{t}, \mathrm{J}=7.0 \mathrm{~Hz}, 3 \mathrm{H})$.

${ }^{13}$ C NMR (101 MHz, CDC13) $\delta: 167.10,152.48,141.80,136.92,136.88,134.46,133.98$, $133.63,131.61,131.55,131.41,131.38,128.07,127.99,125.08,121.26,121.24,120.00,115.59$, $105.80,63.99,63.91,60.66,34.43,33.05,19.57,16.42,16.36,14.46$.

HRMS: $\left[(\mathrm{M}+\mathrm{Na})^{+}\right]$Calcd.: 436.1284; observed: 436.1286 .

IR (thin film): 2983, 2231, 1711, 1601, 1488, 1236, 1183, 1036, $847 \mathrm{~cm}^{-1}$<smiles>CCOC(=O)/C=C/c1cc(CP(=O)(OCC)Oc2ccccc2C#N)ccc1OC</smiles>

(E)-ethyl 3-(5-(((2-cyanophenoxy)(ethoxy)phosphoryl)methyl)-2-methoxyphenyl)acrylate (2n):

Yield : 75\% (64 mg)

Appearance: Liquid

$\mathbf{R}_{\mathbf{f}}: 0.3$ (30\% ethyl acetate/pet-ether mixture)

Isolation: Petroleum ether :ethyl acetate(v/v) (13:7)

${ }^{1}$ H NMR (500 MHz, Chloroform-d) $\delta: 7.90(\mathrm{~d}, \mathrm{~J}=16.1 \mathrm{~Hz}, 1 \mathrm{H}), 7.59$ (dd, J = 7.8, $\left.1.7 \mathrm{~Hz}, 1 \mathrm{H}\right)$, $7.51-7.47(\mathrm{~m}, 1 \mathrm{H}), 7.46-7.44(\mathrm{~m}, 1 \mathrm{H}), 7.34(\mathrm{~d}, \mathrm{~J}=8.4 \mathrm{~Hz}, 2 \mathrm{H}), 7.23-7.18(\mathrm{~m}, 1 \mathrm{H}), 6.86(\mathrm{~d}$, $\mathrm{J}=8.5 \mathrm{~Hz}, 1 \mathrm{H}), 6.50(\mathrm{~d}, \mathrm{~J}=16.3 \mathrm{~Hz}, 1 \mathrm{H}), 4.24(\mathrm{qd}, \mathrm{J}=7.1,0.8 \mathrm{~Hz}, 2 \mathrm{H}), 4.20-4.13(\mathrm{~m}, 2 \mathrm{H})$, $3.85(\mathrm{~s}, 3 \mathrm{H}), 3.37(\mathrm{~d}, \mathrm{~J}=21.3 \mathrm{~Hz}, 2 \mathrm{H}), 1.31(\mathrm{t}, \mathrm{J}=7.1 \mathrm{~Hz}, 3 \mathrm{H}), 1.23(\mathrm{t}, \mathrm{J}=7.1 \mathrm{~Hz}, 3 \mathrm{H})$.

${ }^{13}$ C NMR (126 MHz, CDC13) $\delta: 167.53,157.78,152.52,139.53,134.49,133.64,133.01$, $132.96,130.47,130.41,125.12,123.83,122.05,121.97,121.24,121.22,119.48,115.61,111.72$, $105.77,64.03,63.97,60.53,55.75,33.72,32.61,16.42,16.38,14.48$.

${ }^{31}$ P NMR (202 MHz, CDCl3) $\delta: 24.40$

HRMS: $\left[(\mathrm{M}+\mathrm{Na})^{+}\right]$Calcd.: 452.1214; observed: 452.1214 .<smiles>CCOC(=O)C=Cc1cc(CP(=O)(OCC)Oc2ccccc2C#N)ccc1Cl</smiles>

(E)-ethyl 3-(2-chloro-5-(((2-cyanophenoxy)(ethoxy)phosphoryl)methyl)phenyl)acrylate (2p): 
Yield : 76\% (65 mg)

Appearance: Liquid

$\mathbf{R}_{\mathbf{f}}: 0.3$ (30\% ethyl acetate/pet-ether mixture)

Isolation: Petroleum ether :ethyl acetate(v/v) (13:7)

${ }^{1}$ H NMR (500 MHz, Chloroform-d) $\delta: 8.01(\mathrm{~d}, \mathrm{~J}=16.0 \mathrm{~Hz}, 1 \mathrm{H}), 7.60(\mathrm{dt}, \mathrm{J}=8.2,2.7 \mathrm{~Hz}, 2 \mathrm{H})$, $7.54-7.48(\mathrm{~m}, 1 \mathrm{H}), 7.39-7.34(\mathrm{~m}, 2 \mathrm{H}), 7.33-7.28(\mathrm{~m}, 1 \mathrm{H}), 7.23(\mathrm{t}, \mathrm{J}=7.6 \mathrm{~Hz}, 1 \mathrm{H}), 6.43(\mathrm{~d}, \mathrm{~J}$ $=16.0 \mathrm{~Hz}, 1 \mathrm{H}), 4.26(\mathrm{q}, \mathrm{J}=7.1 \mathrm{~Hz}, 2 \mathrm{H}), 4.22-4.14(\mathrm{~m}, 2 \mathrm{H}), 3.41(\mathrm{~d}, \mathrm{~J}=22.0 \mathrm{~Hz}, 2 \mathrm{H}), 1.33$ (t, $\mathrm{J}=7.1 \mathrm{~Hz}, 3 \mathrm{H}), 1.26(\mathrm{t}, \mathrm{J}=7.1 \mathrm{~Hz}, 3 \mathrm{H})$.

${ }^{13}$ C NMR (126 MHz, $\left.\mathrm{CDCl}_{3}\right) \delta: 166.55,152.30,139.90,134.57,134.17,133.69,133.16$, $132.62,132.57,130.65,130.62,129.44,129.23,129.17,125.31,121.63,121.25,121.23,115.57$, $105.78,64.15,64.09,60.88,34.19,33.08,16.43,16.39,14.43$.

HRMS: $\left[(\mathrm{M}+\mathrm{Na})^{+}\right]$Calcd.: 456.0738; observed: 456.0735 .

IR (thin film): 2986, 2233, 1713, 1488, 1237, 1037, 923, $765 \mathrm{~cm}^{-1}$<smiles>CCOC(=O)C=Cc1cc(CP(=O)(OCC)Oc2ccccc2C#N)ccc1Br</smiles>

(E)-ethyl 3-(2-bromo-5-(((2-cyanophenoxy)(ethoxy)phosphoryl)methyl)phenyl)acrylate (2q):

Yield: $74 \%(70 \mathrm{mg})$

Appearance: Liquid

$\mathbf{R}_{\mathbf{f}}: 0.3$ (30\% ethyl acetate/pet-ether mixture)

Isolation: Petroleum ether :ethyl acetate(v/v) (13:7)

${ }^{1} \mathrm{H}$ NMR (400 MHz, Chloroform-d) $\delta: 7.97$ (d, J = $\left.15.9 \mathrm{~Hz}, 1 \mathrm{H}\right), 7.65-7.45$ (m, 4H), 7.38 (d, J $=8.4 \mathrm{~Hz}, 1 \mathrm{H}), 7.26(\mathrm{~s}, 1 \mathrm{H}), 7.22(\mathrm{~d}, \mathrm{~J}=7.9 \mathrm{~Hz}, 1 \mathrm{H}), 6.39(\mathrm{~d}, \mathrm{~J}=16.0 \mathrm{~Hz}, 1 \mathrm{H}), 4.33-4.10(\mathrm{~m}$, 4H), $3.40(\mathrm{~d}, \mathrm{~J}=22.0 \mathrm{~Hz}, 2 \mathrm{H}), 1.33(\mathrm{t}, \mathrm{J}=7.1 \mathrm{~Hz}, 3 \mathrm{H}), 1.26(\mathrm{t}, \mathrm{J}=7.0 \mathrm{~Hz}, 3 \mathrm{H})$.

${ }^{13} \mathrm{C}$ NMR (126 MHz, $\left.\mathrm{CDCl}_{3}\right) \delta: 166.47,152.39,152.33,142.46,135.08,135.06,134.59$, $133.93,133.91,133.71,132.82,132.76,130.23,130.16,129.41,129.35,125.33,124.45,124.41$, $121.85,121.29,121.27,115.58,105.82,64.19,64.13,60.91,34.29,33.18,16.46,16.41,14.45$.

HRMS: $\left[(\mathrm{M}+\mathrm{Na})^{+}\right]$Calcd.: 500.0224; observed: 500.0219 .

IR (thin film): 2983, 2233, 1712, 1263, 1239, 1036, 920, $763 \mathrm{~cm}^{-1}$ 
<smiles>CCOC(=O)C=Cc1cc(F)cc(CP(=O)(OCC)Oc2ccccc2C#N)c1F</smiles>

(E)-ethyl 3-(3-(((2-cyanophenoxy)(ethoxy)phosphoryl)methyl)-2,5-difluorophenyl)acrylate (2r):

Yield : 84\% (72 mg)

Appearance: Liquid

$\mathbf{R}_{\mathbf{f}}: 0.3$ (30\% ethyl acetate/pet-ether mixture)

Isolation: Petroleum ether :ethyl acetate(v/v) (13:7)

${ }^{1}$ H NMR (500 MHz, Chloroform-d) $\delta: 7.74(\mathrm{dd}, \mathrm{J}=16.2,1.0 \mathrm{~Hz}, 1 \mathrm{H}), 7.63$ (dt, J = 7.9, $1.1 \mathrm{~Hz}$, 1H), $7.58-7.54(\mathrm{~m}, 1 \mathrm{H}), 7.46(\mathrm{dt}, \mathrm{J}=8.4,1.2 \mathrm{~Hz}, 1 \mathrm{H}), 7.26(\mathrm{~s}, 1 \mathrm{H}), 7.23(\mathrm{ddd}, \mathrm{J}=10.8,5.9,1.8$ $\mathrm{Hz}, 1 \mathrm{H}), 7.18$ (ddq, J = 8.3, 5.5, $3.1 \mathrm{~Hz}, 1 \mathrm{H}), 6.49(\mathrm{~d}, \mathrm{~J}=16.2 \mathrm{~Hz}, 1 \mathrm{H}), 4.32-4.22(\mathrm{~m}, 4 \mathrm{H})$, $3.50(\mathrm{~d}, \mathrm{~J}=21.8 \mathrm{~Hz}, 2 \mathrm{H}), 1.34$ (t, J = 7.1 Hz, 3H), 1.29 (t, J=7.1 Hz, 3H).

${ }^{13} \mathbf{C}$ NMR (126 MHz, $\left.\mathrm{CDCl}_{3}\right) \delta: 166.37,152.10,152.04,135.74,134.53,133.76,125.40$, $122.48,122.44,121.07,121.05,120.16,120.08,119.96,119.88,115.32,114.15,113.93,105.86$, $64.36,64.30,60.96,27.68,26.55,16.33,16.28,14.36$.

HRMS: $\left[(\mathrm{M}+\mathrm{Na})^{+}\right]$Calcd.: 458.0939; observed: 458.0937.

IR (thin film): 2985, 2234, 1714, 1488, 1449, 1280, 1184, 1036, 923, $766 \mathrm{~cm}^{-1}$<smiles>CCOCC=Cc1ccc(Br)c(CP(=O)(OCC)Oc2ccccc2C#N)c1</smiles>

(E)-ethyl 3-(4-bromo-3-(((2-cyanophenoxy)(ethoxy)phosphoryl)methyl)phenyl)acrylate (2s):

Yield : 81\% (77 mg)

Appearance: Liquid

$\mathbf{R}_{\mathbf{f}}: 0.3$ (30\% ethyl acetate/pet-ether mixture)

Isolation: Petroleum ether :ethyl acetate(v/v) (13:7)

${ }^{1}$ H NMR (500 MHz, Chloroform-d) $\delta: 7.64$ (q, J = 3.8, $\left.3.2 \mathrm{~Hz}, 1 \mathrm{H}\right), 7.63-7.55$ (m, 3H), 7.51 $7.47(\mathrm{~m}, 1 \mathrm{H}), 7.40(\mathrm{~d}, \mathrm{~J}=8.5 \mathrm{~Hz}, 1 \mathrm{H}), 7.28(\mathrm{~d}, \mathrm{~J}=8.4 \mathrm{~Hz}, 1 \mathrm{H}), 7.22(\mathrm{t}, \mathrm{J}=7.8 \mathrm{~Hz}, 1 \mathrm{H}), 6.43(\mathrm{~d}$, 
$\mathrm{J}=16.0 \mathrm{~Hz}, 1 \mathrm{H}), 4.27-4.16(\mathrm{~m}, 4 \mathrm{H}), 3.69(\mathrm{~d}, \mathrm{~J}=22.3 \mathrm{~Hz}, 2 \mathrm{H}), 1.35-1.29(\mathrm{~m}, 3 \mathrm{H}), 1.27(\mathrm{~d}, \mathrm{~J}$ $=7.0 \mathrm{~Hz}, 3 \mathrm{H})$.

${ }^{13}$ C NMR (126 MHz, $\left.\mathrm{CDCl}_{3}\right) \delta: 166.76,152.23,142.84,134.49,133.87,133.84,133.74$, $133.69,131.44,131.40,128.15,128.12,127.00,126.93,125.32,125.23,121.08,119.76,115.47$, $105.79,64.29,64.24,60.79,34.55,33.44,16.39,16.35,14.42$.

HRMS: $\left[(\mathrm{M}+\mathrm{Na})^{+}\right]$Calcd.: 500.0224; observed: 500.0227 .<smiles>CCOC(=O)C=Cc1ccc(C)c(CP(=O)(OCC)Oc2ccccc2C#N)c1</smiles>

(E)-ethyl 3-(3-(((2-cyanophenoxy)(ethoxy)phosphoryl)methyl)-4-methylphenyl)acrylates (2t):

Yield : 77\% (63 mg)

Appearance: Liquid

$\mathbf{R}_{\mathbf{f}}: 0.3$ (30\% ethyl acetate/pet-ether mixture)

Isolation: Petroleum ether :ethyl acetate(v/v) (13:7)

${ }^{1}$ H NMR $\left(400 \mathrm{MHz}, \mathrm{CDCl}_{3}\right) \delta 7.63(\mathrm{~d}, J=5.4 \mathrm{~Hz}, 1 \mathrm{H}), 7.60(\mathrm{~d}, J=2.9 \mathrm{~Hz}, 1 \mathrm{H}), 7.52-7.45(\mathrm{~m}$, $2 \mathrm{H}), 7.37-7.32(\mathrm{~m}, 1 \mathrm{H}), 7.29(\mathrm{~d}, J=8.6 \mathrm{~Hz}, 1 \mathrm{H}), 7.21(\mathrm{t}, J=7.7 \mathrm{~Hz}, 2 \mathrm{H}), 6.38(\mathrm{~d}, J=16.0 \mathrm{~Hz}$, $1 \mathrm{H}), 4.28-4.21(\mathrm{~m}, 2 \mathrm{H}), 4.19-4.10(\mathrm{~m}, 2 \mathrm{H}), 3.47$ (d, $J=22.2 \mathrm{~Hz}, 2 \mathrm{H}), 2.44$ (s, 3H), 1.33 (t, $J$ $=7.1 \mathrm{~Hz}, 3 \mathrm{H}), 1.23(\mathrm{t}, J=7.1 \mathrm{~Hz}, 3 \mathrm{H})$.

${ }^{13}$ C NMR (101 MHz, $\left.\mathrm{CDCl}_{3}\right) \delta 167.24,152.57,152.48,144.12,140.14,140.07,134.53,133.69$, 132.82 , 132.78, 131.49, 131.46, 130.78, 130.72, 129.52, 129.42, 127.21, 127.18, 125.16, 121.19, $118.04,115.68,105.96,64.06,63.98,60.64,32.25,30.88,20.23,16.41,16.35,14.49$.

HRMS: [(M +Na $\left.)^{+}\right]$Calcd.: 436.1286; observed: 436.1289 .<smiles>CCOC(=O)C=Cc1cc(Br)cc(CP(=O)(OCC)Oc2ccccc2C#N)c1</smiles>

(E)-ethyl 3-(3-bromo-5-(((2-cyanophenoxy)(ethoxy)phosphoryl)methyl)phenyl)acrylate (2u): 
Yield : $82 \%(78 \mathrm{mg})$

Appearance: Liquid

$\mathbf{R}_{\mathbf{f}}: 0.3$ (30\% ethyl acetate/pet-ether mixture)

Isolation: Petroleum ether :ethyl acetate(v/v) (13:7)

${ }^{1}$ H NMR (500 MHz, Chloroform- $d$ ) $\delta: 7.63(\mathrm{~d}, J=7.8 \mathrm{~Hz}, 1 \mathrm{H}), 7.60-7.50(\mathrm{~m}, 4 \mathrm{H}), 7.46$ (s, $1 \mathrm{H}), 7.41(\mathrm{~d}, J=8.5 \mathrm{~Hz}, 1 \mathrm{H}), 7.26(\mathrm{q}, J=8.0 \mathrm{~Hz}, 1 \mathrm{H}), 6.44(\mathrm{~d}, J=16.0 \mathrm{~Hz}, 1 \mathrm{H}), 4.24(\mathrm{dt}, J=$ 23.3, 7.6 Hz, 4H), $3.43(\mathrm{~d}, J=22.0 \mathrm{~Hz}, 2 \mathrm{H}), 1.34$ (t, $J=7.2 \mathrm{~Hz}, 3 \mathrm{H}), 1.28$ (t, $J=7.5 \mathrm{~Hz}, 3 \mathrm{H})$.

${ }^{13} \mathrm{C}$ NMR (126 MHz, $\left.\mathrm{CDCl}_{3}\right) \delta: 166.54,152.30,152.23,142.42,137.00,136.97,134.56$, $134.37,134.32$, 133.70, 133.09, 133.01, 129.87, 129.84, 128.46, 128.41, 125.38, 123.32, 123.29, $121.24,120.50,115.50,105.85,64.26,64.20,60.87,34.30,33.19,16.41,16.36,14.41$.

${ }^{31}$ P NMR (202 MHz, CDC13) $\delta: 22.84$

HRMS: $\left[(\mathrm{M}+\mathrm{Na})^{+}\right]$Calcd.: 500.0224; observed: 500.0220.

IR (thin film): 2986, 2230, 1712, 1640, 1264, 1032, 922, $766 \mathrm{~cm}^{-1}$<smiles>CCOC(=O)C=Cc1cc(C)cc(CP(=O)(OCC)Oc2ccccc2C#N)c1</smiles>

(E)-ethyl 3-(3-(((2-cyanophenoxy)(ethoxy)phosphoryl)methyl)-5-methylphenyl)acrylates (2v):

Yield : 80\% (66 mg)

Appearance: Liquid

$\mathbf{R}_{\mathbf{f}}: 0.3$ (30\% ethyl acetate/pet-ether mixture)

Isolation: Petroleum ether :ethyl acetate(v/v) (13:7)

${ }^{1} \mathbf{H}$ NMR $\left(400 \mathrm{MHz}, \mathrm{CDCl}_{3}\right) \delta 7.60(\mathrm{dd}, J=11.9,4.1 \mathrm{~Hz}, 2 \mathrm{H}), 7.54-7.43(\mathrm{~m}, 1 \mathrm{H}), 7.34(\mathrm{~d}, J=$ $8.5 \mathrm{~Hz}, 1 \mathrm{H}), 7.30(\mathrm{~s}, 1 \mathrm{H}), 7.25-7.18(\mathrm{~m}, 3 \mathrm{H}), 6.40(\mathrm{~d}, J=16.0 \mathrm{~Hz}, 1 \mathrm{H}), 4.40-4.13(\mathrm{~m}, 4 \mathrm{H})$, $3.42(\mathrm{~d}, J=21.9 \mathrm{~Hz}, 2 \mathrm{H}), 2.33(\mathrm{~s}, 3 \mathrm{H}), 1.33(\mathrm{t}, J=7.1 \mathrm{~Hz}, 3 \mathrm{H}), 1.26(\mathrm{t}, J=7.1 \mathrm{~Hz}, 3 \mathrm{H})$.

${ }^{13}$ C NMR (101 MHz, $\left.\mathrm{CDCl}_{3}\right) \delta 167.10,152.40,152.32,144.26,139.34,135.04,134.51,134.27$, $133.67,133.16,132.86,132.75,130.59,130.50,127.99,126.88,126.82,125.15,121.18,121.13$, $120.10,118.78,116.38,115.58,105.69,64.18,64.10,60.67,34.59,33.22,21.30,16.37,16.31$, 14.42 .

HRMS: $\left[(\mathrm{M}+\mathrm{Na})^{+}\right]$Calcd.: 436.1286; observed: 436.1287 . 
<smiles>CCOC(=O)C=Cc1cc(CP(=O)(OCC)Oc2ccccc2C#N)cc(Br)c1F</smiles>

(E)-ethyl 3-(3-bromo-5-(((2-cyanophenoxy)(ethoxy)phosphoryl)methyl)-2fluorophenyl)27crylates (2w):

Yield : 75\% (75 mg)

Appearance: Liquid

$\mathbf{R}_{\mathbf{f}}: 0.3$ (30\% ethyl acetate/pet-ether mixture)

Isolation: Petroleum ether :ethyl acetate(v/v) (13:7)

${ }^{1}$ H NMR (400 MHz, Chloroform- $d$ ) $\delta: 7.72(\mathrm{~d}, J=16.2 \mathrm{~Hz}, 1 \mathrm{H}), 7.61(\mathrm{dt}, J=7.8,1.2 \mathrm{~Hz}, 1 \mathrm{H})$, $7.58-7.50(\mathrm{~m}, 2 \mathrm{H}), 7.47(\mathrm{dt}, J=5.5,2.5 \mathrm{~Hz}, 1 \mathrm{H}), 7.41(\mathrm{dt}, J=8.6,1.2 \mathrm{~Hz}, 1 \mathrm{H}), 7.28-7.24(\mathrm{~m}$, $1 \mathrm{H}), 6.54(\mathrm{~d}, J=16.2 \mathrm{~Hz}, 1 \mathrm{H}), 4.28-4.18(\mathrm{~m}, 4 \mathrm{H}), 3.41(\mathrm{~d}, J=21.7 \mathrm{~Hz}, 2 \mathrm{H}), 1.32(\mathrm{t}, J=7.1$ $\mathrm{Hz}, 3 \mathrm{H}), 1.28$ (t, $J=7.1 \mathrm{~Hz}, 3 \mathrm{H})$.

${ }^{13}$ C NMR (101 MHz, $\left.\mathrm{CDCl}_{3}\right) \delta: 166.44,152.17,136.07,136.00,135.90,134.60,133.71$, $129.59,129.56,129.49,127.84,127.79,125.45,122.84,122.78,121.26,121.23,115.49,110.38$, $105.87,64.30,64.22,60.98,33.82,32.42,16.44,16.38,14.41$.

${ }^{31} \mathbf{P}$ NMR $\left(202 \mathrm{MHz}, \mathrm{CDCl}_{3}\right) \delta: 22.75$

HRMS: $\left[(\mathrm{M}+\mathrm{Na})^{+}\right]$Calcd.: 518.0134; observed: 518.0136.

IR (thin film): 2986, 2233, 1714, 1488, 1273, 1184, 1037, 923, $765 \mathrm{~cm}^{-1}$

\section{Homo-diolefination and Hetero di-olefination:}<smiles>CCOCC=Cc1cc(C=CC(=O)OCC)cc(CP(=O)(OCC)Oc2ccccc2C#N)c1</smiles>

(2E,2'E)-diethyl 3,3'-(5-(((2-cyanophenoxy)(ethoxy)phosphoryl)methyl)-1,3phenylene)diacrylate (3a):

Yield : $85 \%(85 \mathrm{mg})$

Appearance: Liquid

$\mathbf{R}_{\mathbf{f}}: 0.3$ (40\% ethyl acetate/pet-ether mixture)

Isolation: Petroleum ether : ethyl acetate(v/v) (3:2) 
${ }^{1}$ H NMR (500 MHz, Chloroform-d) $\delta: 7.63(\mathrm{~d}, \mathrm{~J}=16.1 \mathrm{~Hz}, 2 \mathrm{H}), 7.60(\mathrm{dt}, \mathrm{J}=7.7,1.2 \mathrm{~Hz}, 1 \mathrm{H})$, $7.54(\mathrm{~d}, \mathrm{~J}=1.8 \mathrm{~Hz}, 1 \mathrm{H}), 7.53(\mathrm{~d}, \mathrm{~J}=2.4 \mathrm{~Hz}, 2 \mathrm{H}), 7.51-7.48(\mathrm{~m}, 1 \mathrm{H}), 7.41-7.37(\mathrm{~m}, 1 \mathrm{H}), 7.22$ $(\mathrm{t}, \mathrm{J}=7.5 \mathrm{~Hz}, 1 \mathrm{H}), 6.46(\mathrm{~d}, \mathrm{~J}=16.0 \mathrm{~Hz}, 2 \mathrm{H}), 4.26(\mathrm{q}, \mathrm{J}=7.1 \mathrm{~Hz}, 4 \mathrm{H}), 4.22-4.11(\mathrm{~m}, 2 \mathrm{H}), 3.46$ $(\mathrm{d}, \mathrm{J}=22.0 \mathrm{~Hz}, 2 \mathrm{H}), 1.33(\mathrm{t}, \mathrm{J}=7.1 \mathrm{~Hz}, 6 \mathrm{H}), 1.26(\mathrm{t}, \mathrm{J}=7.0 \mathrm{~Hz}, 3 \mathrm{H})$.

${ }^{13}$ C NMR $\left(126 \mathrm{MHz}, \mathrm{CDCl}_{3}\right) \delta: 166.75,152.31,143.22,135.89,134.56,133.70,131.98$, $131.91,131.03,130.97,126.73,126.70,125.33,121.29,121.27,120.09,115.55,105.90,64.21$, $64.15,60.85,34.62,33.51,16.46,16.41,14.46$.

HRMS: $\left[(\mathrm{M}+\mathrm{Na})^{+}\right]$Calcd.: 520.1496; observed: 520.1492 .<smiles>CCOP(=O)(Cc1cc(/C=C/C(C)=O)cc(/C=C/C(C)=O)c1)Oc1ccccc1C#N</smiles>

2-cyanophenyl ethyl 3,5-bis((E)-3-oxobut-1-en-1-yl)benzylphosphonate (3b):

Yield : 78\% $(67 \mathrm{mg})$

Appearance: Liquid

$\mathbf{R}_{\mathbf{f}}: 0.2$ ( $40 \%$ ethyl acetate/pet-ether mixture)

Isolation: Petroleum ether :ethyl acetate(v/v) (1:1)

${ }^{1}$ H NMR (500 MHz, Chloroform-d) $8: 7.63-7.59$ (m, 2H), 7.58 (dd, J = 2.8, 1.5 Hz, 2H), 7.54 $-7.51(\mathrm{~m}, 1 \mathrm{H}), 7.49(\mathrm{~d}, \mathrm{~J}=16.5 \mathrm{~Hz}, 2 \mathrm{H}), 7.42(\mathrm{dt}, \mathrm{J}=8.5,1.2 \mathrm{~Hz}, 1 \mathrm{H}), 7.23$ (tt, J = 7.6, 0.8 Hz, $1 \mathrm{H}), 6.75(\mathrm{~d}, \mathrm{~J}=16.3 \mathrm{~Hz}, 2 \mathrm{H}), 4.25-4.15(\mathrm{~m}, 2 \mathrm{H}), 3.48(\mathrm{~d}, \mathrm{~J}=22.0 \mathrm{~Hz}, 2 \mathrm{H}), 2.38(\mathrm{~s}, 6 \mathrm{H}), 1.27$ (t, J $=7.1 \mathrm{~Hz}, 3 \mathrm{H})$.

${ }^{13}$ C NMR (126 MHz, CDC13) $\delta: 198.28,152.42,141.90,136.02,136.00,134.63,134.50$, $133.77,132.13,132.05,131.51,131.45,128.48,127.04,127.01,125.38,121.16,121.14,116.54$, $115.64,105.75,64.32,64.26,34.62,33.51,28.01,16.48,16.43$.

HRMS: $\left[(\mathrm{M}+\mathrm{Na})^{+}\right]$Calcd.: 460.1284; observed: 460.1288 .<smiles>CCOP(=O)(Cc1cc(C2CCCC=C2C(C)=O)cc(C2CCCC=C2C(C)=O)c1)Oc1ccccc1C#N</smiles>

2-cyanophenyl ethyl ((6,6"'-diacetyl-1,1',2,2",3,3",4,4"'-octahydro-[1,1':3',1'-terphenyl]5 '-yl)methyl)phosphonate (3c): 
Yield : 69\% (75 mg)

Appearance: Liquid

$\mathbf{R}_{\mathbf{f}}: 0.3$ (40\% ethyl acetate/pet-ether mixture)

Isolation: Petroleum ether :ethyl acetate(v/v) $(3: 2)$

${ }^{1}$ H NMR (400 MHz, Chloroform-d) $\delta: 7.52(\mathrm{dt}, \mathrm{J}=8.1,2.3 \mathrm{~Hz}, 1 \mathrm{H}), 7.42$ (dtd, J = 8.9, 7.3, 1.8 $\mathrm{Hz}, 1 \mathrm{H}), 7.12$ (dt, J = 11.3, $3.5 \mathrm{~Hz}, 3 \mathrm{H}), 6.94-6.66(\mathrm{~m}, 3 \mathrm{H}), 6.56(\mathrm{dd}, \mathrm{J}=39.8,8.5 \mathrm{~Hz}, 1 \mathrm{H})$, 4.14 (dddd, $\mathrm{J}=12.3,10.1,8.2,6.0 \mathrm{~Hz}, 2 \mathrm{H}), 3.93$ (q, J = 5.6, $4.9 \mathrm{~Hz}, 2 \mathrm{H}), 3.39-3.25(\mathrm{~m}, 2 \mathrm{H})$, $2.45-2.34(\mathrm{~m}, 2 \mathrm{H}), 2.25(\mathrm{ttt}, \mathrm{J}=8.0,5.7,3.0 \mathrm{~Hz}, 1 \mathrm{H}), 2.20-2.09(\mathrm{~m}, 5 \mathrm{H}), 1.77$ (tdt, J = 15.4, $13.2,6.3 \mathrm{~Hz}, 2 \mathrm{H}), 1.68-1.47(\mathrm{~m}, 2 \mathrm{H}), 1.47-1.34(\mathrm{~m}, 3 \mathrm{H}), 1.24$ (q, J = 7.2 Hz, 4H).

${ }^{13} \mathrm{C}$ NMR (126 MHz, $\left.\mathrm{CDCl}_{3}\right) \delta: 198.89,198.65,152.52,145.96,145.75,142.19,142.09$, $141.01,140.86,134.82,134.71,133.23,133.17,129.25,127.75,127.56,127.51,127.19,127.01$, $124.75,121.31,121.29,115.81,105.26,63.69,63.63,38.55,38.37,34.80,33.73,26.21,26.19$, $26.04,25.93,17.06,16.83,16.37,16.31$.

HRMS: $\left[(\mathrm{M}+\mathrm{Na})^{+}\right]$Calcd.: 568.2223; observed: 568.2220.

IR (thin film): 2831, 2233, 1667, 1239, 1029, 924, $768 \mathrm{~cm}^{-1}$

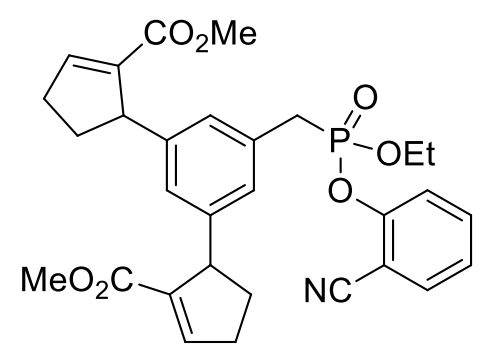

dimethyl

5,5'-(5-(((2-cyanophenoxy)(ethoxy)phosphoryl)methyl)-1,3phenylene)bis(cyclopent-1-enecarboxylate) (3d):

Yield : 72\% (78 mg)

Appearance: Liquid

$\mathbf{R}_{\mathbf{f}}: 0.3$ (40\% ethyl acetate/pet-ether mixture)

Isolation: Petroleum ether : ethyl acetate(v/v) (3:2)

${ }^{1}$ H NMR (400 MHz, Chloroform-d) $\delta: ~ 7.59-7.53(\mathrm{~m}, 1 \mathrm{H}), 7.48$ - $7.36(\mathrm{~m}, 1 \mathrm{H}), 7.20$ - 7.12 (m, $1 \mathrm{H}), 7.04-6.89(\mathrm{~m}, 5 \mathrm{H}), 6.86(\mathrm{q}, \mathrm{J}=2.1 \mathrm{~Hz}, 1 \mathrm{H}), 4.21-4.10(\mathrm{~m}, 2 \mathrm{H}), 4.10-4.02(\mathrm{~m}, 2 \mathrm{H})$, $3.55(\mathrm{~d}, \mathrm{~J}=5.6 \mathrm{~Hz}, 6 \mathrm{H}), 3.36(\mathrm{dd}, \mathrm{J}=21.8,4.6 \mathrm{~Hz}, 2 \mathrm{H}), 2.61(\mathrm{ddt}, \mathrm{J}=17.3,8.9,2.8 \mathrm{~Hz}, 1 \mathrm{H})$, $2.46(\mathrm{ttd}, \mathrm{J}=13.1,6.5,5.8,3.7 \mathrm{~Hz}, 3 \mathrm{H}), 2.00-1.69(\mathrm{~m}, 4 \mathrm{H}), 1.28-1.16(\mathrm{~m}, 3 \mathrm{H})$.

${ }^{13}$ C NMR (126 MHz, $\left.\mathrm{CDCl}_{3}\right) \delta: 165.27,165.24,152.71,146.02,145.99,145.29,145.24$, $139.16,139.13,134.55,134.48,134.42,133.45,133.44,129.97,126.79,126.62,126.50,125.28$, $124.83,121.36,115.70,105.72,63.82,63.76,51.40,51.37,50.04,50.01,34.81,34.20,33.71$, $32.34,16.38,16.34$. 
HRMS: $\left[(\mathrm{M}+\mathrm{Na})^{+}\right]$Calcd.: 572.1809; observed: 572.1804 .

IR (thin film): 2951, 2233, 1718, 1449, 1280, 1236, 1032, 922, $767 \mathrm{~cm}^{-1}$<smiles>CCOC=Cc1cc(C=CC(C)=O)cc(CP(=O)(OCC)Oc2ccccc2C#N)c1</smiles>

(E)-ethyl 3-(3-(((2-cyanophenoxy)(ethoxy)phosphoryl)methyl)-5-((E)-3-oxobut-1-en-1yl)phenyl)30crylates (3e):

Yield : $81 \%(78 \mathrm{mg})$

Appearance: Liquid

$\mathbf{R}_{\mathbf{f}}: 0.3$ ( $40 \%$ ethyl acetate/pet-ether mixture)

Isolation: Petroleum ether :ethyl acetate(v/v) (3:2)

${ }^{1} \mathbf{H}$ NMR (500 MHz, Chloroform-d) $\delta: 7.67-7.59(\mathrm{~m}, 2 \mathrm{H}), 7.57(\mathrm{~d}, \mathrm{~J}=2.2 \mathrm{~Hz}, 2 \mathrm{H}), 7.55-7.44$ (m, 3H), 7.40 (dd, J = 8.7, $2.5 \mathrm{~Hz}, 1 \mathrm{H}), 7.23$ (td, J = 7.7, $2.5 \mathrm{~Hz}, 1 \mathrm{H}), 6.74(\mathrm{dd}, \mathrm{J}=16.3,2.5 \mathrm{~Hz}$, 1H), 6.47 (dd, J = 16.0, $2.5 \mathrm{~Hz}, 1 \mathrm{H}), 4.33-4.17(\mathrm{~m}, 4 \mathrm{H}), 3.47(\mathrm{dd}, \mathrm{J}=22.0,2.5 \mathrm{~Hz}, 2 \mathrm{H}), 2.38$ $(\mathrm{d}, \mathrm{J}=2.6 \mathrm{~Hz}, 3 \mathrm{H}), 1.36-1.31(\mathrm{~m}, 3 \mathrm{H}), 1.30-1.25(\mathrm{~m}, 3 \mathrm{H})$.

${ }^{13} \mathbf{C}$ NMR $\left(126 \mathrm{MHz}, \mathrm{CDCl}_{3}\right) \delta: 198.25,166.73,152.40,143.14,141.97,135.93,135.91$, $134.58,133.72,132.05,131.97,131.28,131.23,131.18,128.41,126.87,126.85,125.35,121.21$, $121.19,120.16,115.59,105.76,64.25,64.19,60.87,34.60,33.50,27.98,16.46,16.41,14.45$.

HRMS: $\left[(\mathrm{M}+\mathrm{Na})^{+}\right]$Calcd.: 490.1390; observed: 490.1382 .<smiles>CCOC=Cc1cc(C=CC(C)=O)cc(CP(=O)(OCC)Oc2ccccc2C#N)c1</smiles>

(E)-ethyl 3-(3-((2-cyanophenoxy)(ethoxy)phosphoryl)methyl)-5-((E)-3-methoxy-3oxoprop-1-en-1-yl)phenyl)30crylates (3f):

Yield : $82 \%(79 \mathrm{mg})$

Appearance: Liquid

$\mathbf{R}_{\mathbf{f}}: 0.3$ ( $40 \%$ ethyl acetate/pet-ether mixture)

Isolation: Petroleum ether :ethyl acetate(v/v) (3:2)

${ }^{1}$ H NMR $(500 \mathrm{MHz}$, Chloroform- $d$ ) $\delta: 7.66(\mathrm{~d}, J=4.9 \mathrm{~Hz}, 1 \mathrm{H}), 7.61(\mathrm{dd}, J=11.6,6.4 \mathrm{~Hz}, 2 \mathrm{H})$, $7.56-7.49$ (m, 4H), 7.39 (d, $J=8.5 \mathrm{~Hz}, 1 \mathrm{H}), 7.22$ (t, $J=7.6 \mathrm{~Hz}, 1 \mathrm{H}), 6.46(\mathrm{dd}, J=16.1,3.1 \mathrm{~Hz}$, 
2H), $4.26(\mathrm{q}, J=7.1 \mathrm{~Hz}, 2 \mathrm{H}), 4.23-4.15(\mathrm{~m}, 2 \mathrm{H}), 3.81(\mathrm{~s}, 3 \mathrm{H}), 3.47(\mathrm{~d}, J=22.0 \mathrm{~Hz}, 2 \mathrm{H}), 1.33$ (t, $J=7.1 \mathrm{~Hz}, 3 \mathrm{H}), 1.27(\mathrm{t}, J=7.3 \mathrm{~Hz}, 3 \mathrm{H})$.

${ }^{13} \mathrm{C}$ NMR (126 MHz, $\left.\mathrm{CDCl}_{3}\right) \delta: 167.21,166.75,152.37,143.54,143.20,135.93,135.90$, $135.82,135.80,134.56,133.70,132.00,131.93,131.09,131.03,131.01,130.95,126.81,126.78$, $125.33,121.26,121.24,120.11,119.58,115.57,105.82,64.20,64.14,60.86,52.02,34.61$, $33.51,16.46,16.41,14.47$.

HRMS: $\left[(\mathrm{M}+\mathrm{Na})^{+}\right]$Calcd.: 506.1339; observed: 506.1338.

IR (thin film): 2925, 2233, 1713, 1640, 1447, 1173, 1036, 919, $785 \mathrm{~cm}^{-1}$<smiles>CCOP(=O)(Cc1cc(/C=C/C(C)=O)cc(/C=C/C(C)=O)c1)Oc1ccccc1C#N</smiles>

(E)-methyl 3-(3-(((2-cyanophenoxy)(ethoxy)phosphoryl)methyl)-5-((E)-3-oxobut-1-en-1yl)phenyl)31 crylates (3g):

Yield : 84\% (75 mg)

Appearance: Liquid

$\mathbf{R}_{\mathbf{f}}: 0.3$ (40\% ethyl acetate/pet-ether mixture)

Isolation: Petroleum ether :ethyl acetate(v/v) $(3: 2)$

${ }^{1}$ H NMR (500 MHz, Chloroform- $d$ ) $\delta: 7.65(\mathrm{~d}, J=16.1 \mathrm{~Hz}, 1 \mathrm{H}), 7.62-7.57(\mathrm{~m}, 3 \mathrm{H}), 7.54-$ $7.51(\mathrm{~m}, 2 \mathrm{H}), 7.47-7.38(\mathrm{~m}, 2 \mathrm{H}), 7.22(\mathrm{~d}, J=7.7 \mathrm{~Hz}, 1 \mathrm{H}), 6.74(\mathrm{~d}, J=16.3 \mathrm{~Hz}, 1 \mathrm{H}), 6.47(\mathrm{~d}, J$ $=16.0 \mathrm{~Hz}, 1 \mathrm{H}), 4.21(\mathrm{dtd}, J=8.3,7.0,1.5 \mathrm{~Hz}, 2 \mathrm{H}), 3.81(\mathrm{~s}, 3 \mathrm{H}), 3.48(\mathrm{~d}, J=22.0 \mathrm{~Hz}, 2 \mathrm{H}), 2.38$ (s, 3H), 1.27 (t, $J=7.2 \mathrm{~Hz}, 3 \mathrm{H})$.

${ }^{13}$ C NMR (126 MHz, $\left.\mathrm{CDCl}_{3}\right) \delta: 198.33,167.20,152.29,143.45,141.97,135.93,135.91$, $135.88,135.85,134.60,133.74,132.04,131.96,131.35,131.29,131.22,131.17,128.42,126.96$, $126.94,125.37,121.18,121.16,119.64,115.60,105.77,64.28,64.22,52.05,34.59,33.48$, $27.99,16.46,16.41$.

${ }^{31} \mathbf{P}$ NMR (202 MHz, CDCl3) $\delta: 23.23$

HRMS: $\left[(\mathrm{M}+\mathrm{Na})^{+}\right]$Calcd.: 476.1233; observed: 476.1231.<smiles>CCOP(=O)(Cc1cc(/C=C/COC)cc(/C=C/COC)c1)Oc1ccccc1C#N</smiles> 
(E)-methyl

3-(3-(((2-cyanophenoxy)(ethoxy)phosphoryl)methyl)-5-((E)-2-

(methylsulfonyl)vinyl)phenyl)32crylates (3h):

Yield : 78\% (75 mg)

Appearance: Liquid

$\mathbf{R}_{\mathbf{f}}: 0.3$ (40\% ethyl acetate/pet-ether mixture)

Isolation: Petroleum ether :ethyl acetate(v/v) $(3: 2)$

${ }^{1}$ H NMR (500 MHz, Chloroform- $d$ ) $\delta: 7.60$ (ddd, $\left.J=15.2,7.4,3.6 \mathrm{~Hz}, 5 \mathrm{H}\right), 7.55$ - 7.50 (m, 2H), $7.43(\mathrm{~d}, J=8.7 \mathrm{~Hz}, 1 \mathrm{H}), 7.23(\mathrm{~d}, J=7.7 \mathrm{~Hz}, 1 \mathrm{H}), 7.02(\mathrm{~d}, J=15.4 \mathrm{~Hz}, 1 \mathrm{H}), 6.48(\mathrm{~d}, J=$ $16.1 \mathrm{~Hz}, 1 \mathrm{H}), 4.22$ (dddd, $J=18.7,12.0,5.0,3.2 \mathrm{~Hz}, 2 \mathrm{H}), 3.82$ (s, 3H), 3.49 (d, $J=22.0 \mathrm{~Hz}$, 2H), 3.04 (s, 3H), 1.29 (t, J = 7.3 Hz, 3H).

${ }^{13} \mathrm{C}$ NMR (126 MHz, $\left.\mathrm{CDCl}_{3}\right) \delta$ : 167.08, 152.36, 143.04, 142.54, 139.47, 136.16, 136.13, $134.66,133.79,132.47,132.39,132.03,131.97,131.09,131.03,128.20,127.64,127.61,125.42$, 121.12 , 121.10, 120.08, 115.64, 105.65, 64.34, 64.28, 52.12, 43.35, 34.66, 33.55, 16.50, 16.46 .

${ }^{31} \mathbf{P}$ NMR $\left(202 \mathrm{MHz}, \mathrm{CDCl}_{3}\right) \delta: 22.91$

HRMS: $\left[(\mathrm{M}+\mathrm{Na})^{+}\right]$Calcd.: 512.0903; observed: 512.0908 .

\section{Meta-Hydroxylation:}<smiles>CCOP(=O)(Cc1cccc(O)c1)Oc1ccccc1C#N</smiles>

2-cyanophenyl ethyl 3-hydroxybenzylphosphonate (4a):

Yield : 70\% (44 mg)

Appearance: Liquid

$\mathbf{R}_{\mathbf{f}}: 0.3$ (50\% ethyl acetate/pet-ether mixture)

Isolation: Petroleum ether :ethyl acetate(v/v) $(1: 1)$

${ }^{1}$ H NMR (500 MHz, Chloroform-d) $\delta: 7.59(\mathrm{dd}, \mathrm{J}=7.7,1.6 \mathrm{~Hz}, 1 \mathrm{H}), 7.48(\mathrm{td}, \mathrm{J}=8.1,7.7,1.8$ $\mathrm{Hz}, 1 \mathrm{H}), 7.30$ (d, J = 8.5 Hz, 1H), 7.20 (t, J = 7.6 Hz, 1H), 7.13 (t, J = 7.8 Hz, 1H), 6.97 (q, J = 
$2.2 \mathrm{~Hz}, 1 \mathrm{H}), 6.84-6.77(\mathrm{~m}, 1 \mathrm{H}), 6.74(\mathrm{dt}, \mathrm{J}=8.3,2.5 \mathrm{~Hz}, 1 \mathrm{H}), 4.21-4.11(\mathrm{~m}, 2 \mathrm{H}), 3.39(\mathrm{~d}, \mathrm{~J}=$ $21.7 \mathrm{~Hz}, 2 \mathrm{H}), 1.24(\mathrm{t}, \mathrm{J}=7.0 \mathrm{~Hz}, 3 \mathrm{H})$.

${ }^{13}$ C NMR (126 MHz, $\left.\mathrm{CDCl}_{3}\right) \delta: 157.01,152.44,152.37,134.66,133.68,130.82,130.74$, $130.10,130.07,125.22,121.85,121.79,121.23,121.20,117.21,117.16,115.78,115.13,115.10$, $105.59,64.46,64.40,34.56,33.46,16.36,16.31$.

${ }^{31}$ P NMR (202 MHz, $\left.\mathrm{CDCl}_{3}\right) \delta: 24.70$

HRMS: $\left[(\mathrm{M}+\mathrm{Na})^{+}\right]$Calcd.: 340.0708; observed: 340.0707 .<smiles>CCOP(=O)(Cc1ccc(C)c(O)c1)Oc1ccccc1C#N</smiles>

2-cyanophenyl ethyl 3-hydroxy-4-methylbenzylphosphonate (4b):

Yield : 71\% (47 mg)

Appearance: Liquid

$\mathbf{R}_{\mathbf{f}}: 0.3$ (50\% ethyl acetate/pet-ether mixture)

Isolation: Petroleum ether :ethyl acetate(v/v) $(1: 1)$

${ }^{1}$ H NMR (500 MHz, Chloroform-d) $\delta: 7.58$ (dd, J = 7.8, 1.6 Hz, 1H), 7.47 (ddd, J = 9.0, 7.6, 1.8 $\mathrm{Hz}, 1 \mathrm{H}), 7.33$ (d, J = 8.5 Hz, 1H), 7.19 (t, J = 7.6 Hz, 1H), 7.00 (d, J = 7.6 Hz, 1H), 6.97 (t, J = $2.2 \mathrm{~Hz}, 1 \mathrm{H}), 6.71(\mathrm{dt}, \mathrm{J}=7.7,2.1 \mathrm{~Hz}, 1 \mathrm{H}), 4.16(\mathrm{dq}, \mathrm{J}=8.5,7.1 \mathrm{~Hz}, 2 \mathrm{H}), 3.36(\mathrm{dd}, \mathrm{J}=21.6,1.9$ $\mathrm{Hz}, 2 \mathrm{H}), 2.17$ (d, J = 2.5 Hz, 3H), 1.24 (t, J = 7.1 Hz, 3H).

${ }^{13} \mathrm{C}$ NMR (126 MHz, $\left.\mathrm{CDCl}_{3}\right) \delta: 155.12,152.49,152.42,134.61,133.62,131.25,131.22$, $127.83,127.75,125.10,124.10,124.06,121.61,121.55,121.24,121.22,116.59,116.54,115.86$, $105.50,64.42,64.36,34.21,33.10,16.36,16.31,15.82$.

HRMS: $\left[(\mathrm{M}+\mathrm{Na})^{+}\right]$Calcd.: 354.0866; observed: 354.0863 .<smiles>CCOP(=O)(Cc1ccc(Cl)c(O)c1)Oc1ccccc1C#N</smiles>

2-cyanophenyl ethyl 4-chloro-3-hydroxybenzylphosphonate (4c):

Yield : 62\% (43 mg)

Appearance: Liquid 
$\mathbf{R}_{\mathbf{f}}: 0.3$ (50\% ethyl acetate/pet-ether mixture)

Isolation: Petroleum ether :ethyl acetate(v/v) $(1: 1)$

${ }^{1}$ H NMR (500 MHz, Chloroform-d) $\delta: 7.60$ (dt, J = 7.7, $\left.1.2 \mathrm{~Hz}, 1 \mathrm{H}\right), 7.52$ (ddd, J = 9.0, 7.6, 1.7 $\mathrm{Hz}, 1 \mathrm{H}), 7.38(\mathrm{dd}, \mathrm{J}=8.4,1.2 \mathrm{~Hz}, 1 \mathrm{H}), 7.25-7.20(\mathrm{~m}, 2 \mathrm{H}), 7.07$ (t, J = 2.5 Hz, 1H), 6.83 (dt, J $=8.1,2.5 \mathrm{~Hz}, 1 \mathrm{H}), 4.18(\mathrm{dqd}, \mathrm{J}=11.1,7.1,2.8 \mathrm{~Hz}, 2 \mathrm{H}), 3.38(\mathrm{~d}, \mathrm{~J}=21.9 \mathrm{~Hz}, 2 \mathrm{H}), 1.26(\mathrm{t}, \mathrm{J}=$ $7.0 \mathrm{~Hz}, 3 \mathrm{H})$.

${ }^{13} \mathrm{C}$ NMR (126 MHz, $\left.\mathrm{CDCl}_{3}\right) \delta: 152.40,152.12,134.64,133.73,130.27,130.19,129.76$, $129.73,125.29,122.86,122.80,121.28,121.26,119.71,119.68,118.13,118.08,115.69,105.68$, $64.39,64.33,34.29,33.18,16.40,16.36$.

HRMS: $\left[(\mathrm{M}+\mathrm{K})^{+}\right]$Calcd.: 390.0059; observed: 390.0058 .<smiles>CCOP(=O)(Cc1ccc(Br)c(O)c1)Oc1ccccc1C#N</smiles>

\section{2-cyanophenyl ethyl 4-bromo-3-hydroxybenzylphosphonate (4d):}

Yield : 67\% (53 mg)

Appearance: Liquid

$\mathbf{R}_{\mathbf{f}}: 0.3$ (50\% ethyl acetate/pet-ether mixture)

Isolation: Petroleum ether :ethyl acetate(v/v) $(1: 1)$

${ }^{1}$ H NMR (400 MHz, Chloroform-d) $\delta: 7.60$ (dt, J = 7.8, 1.2 Hz, 1H), 7.52 (ddd, J = 9.1, 7.6, 1.7 $\mathrm{Hz}, 1 \mathrm{H}), 7.37$ (ddd, J = 9.3, 7.9, $1.1 \mathrm{~Hz}, 2 \mathrm{H}), 7.23$ (t, J = 7.6 Hz, 1H), 7.07 (t, J = 2.5 Hz, 1H), $6.76(\mathrm{dt}, \mathrm{J}=8.2,2.4 \mathrm{~Hz}, 1 \mathrm{H}), 4.18(\mathrm{dqd}, \mathrm{J}=8.2,7.1,1.3 \mathrm{~Hz}, 2 \mathrm{H}), 3.37$ (d, J = 22.1 Hz, 2H), 1.25 (t, J $=7.1 \mathrm{~Hz}, 3 \mathrm{H})$.

${ }^{13} \mathbf{C}$ NMR (101 MHz, $\left.\mathrm{CDCl}_{3}\right) \delta: 153.22,153.19,152.27,134.66,133.72,132.85,132.82$, $130.96,130.86,125.32,123.26,123.18,121.27,121.25,117.96,117.89,115.70,109.52,109.47$, $105.64,64.45,64.37,34.42,33.03,16.41,16.35$.

HRMS: $\left[(\mathrm{M}+\mathrm{Na})^{+}\right]$Calcd.: 417.9814; observed: 417.9811.<smiles>CCOP(=O)(Cc1cc(F)cc(O)c1F)Oc1ccccc1C#N</smiles>

2-cyanophenyl ethyl 2,5-difluoro-3-hydroxybenzylphosphonate (4e):

Yield : $62 \%(44 \mathrm{mg})$

Appearance: Liquid 
$\mathbf{R}_{\mathbf{f}}: 0.3$ (50\% ethyl acetate/pet-ether mixture)

Isolation: Petroleum ether :ethyl acetate(v/v) $(1: 1)$

${ }^{1}$ H NMR (400 MHz, Chloroform-d) $\delta: 7.63$ (dq, J = 7.7, 1.6, 1.0 Hz, 1H), 7.55 (ddd, J = 9.0, 7.5, $1.7 \mathrm{~Hz}, 1 \mathrm{H}), 7.46-7.42(\mathrm{~m}, 1 \mathrm{H}), 7.28-7.22(\mathrm{~m}, 1 \mathrm{H}), 6.60(\mathrm{dtd}, \mathrm{J}=9.3,4.8,4.1,2.9 \mathrm{~Hz}, 2 \mathrm{H})$, $4.27-4.15(\mathrm{~m}, 2 \mathrm{H}), 3.43(\mathrm{dd}, \mathrm{J}=22.0,1.3 \mathrm{~Hz}, 2 \mathrm{H}), 1.29(\mathrm{t}, \mathrm{J}=7.1 \mathrm{~Hz}, 3 \mathrm{H})$.

${ }^{13} \mathrm{C}$ NMR (126 MHz, $\left.\mathrm{CDCl}_{3}\right) \delta: 152.16,152.09,145.48,134.69,133.85,125.46,121.18$, $121.12,121.10,115.38,108.47,108.31,108.28,105.75,104.90,104.69,64.63,64.57,27.75$, $26.61,16.38,16.33$.

HRMS: $\left[(\mathrm{M}+\mathrm{Na})^{+}\right]$Calcd.: 376.0521; observed: 376.0529 .

IR (thin film): 2925, 2237, 1601, 1489, 1235, 1037, 926, $764 \mathrm{~cm}^{-1}$<smiles>CCOP(=O)(Cc1cc(O)cc(Br)c1)Oc1ccccc1C#N</smiles>

\section{2-cyanophenyl ethyl 3-bromo-5-hydroxybenzylphosphonate (4f):}

Yield : 61\% (48 mg)

Appearance: Liquid

$\mathbf{R}_{\mathbf{f}}: 0.3$ (50\% ethyl acetate/pet-ether mixture)

Isolation: Petroleum ether :ethyl acetate(v/v) $(1: 1)$

${ }^{1}$ H NMR (500 MHz, Chloroform- $d$ ) $\delta: 7.64(\mathrm{~d}, J=7.9 \mathrm{~Hz}, 1 \mathrm{H}), 7.55$ (q, $\left.J=8.8,8.3 \mathrm{~Hz}, 1 \mathrm{H}\right)$, $7.51-7.36(\mathrm{~m}, 2 \mathrm{H}), 7.31-7.17(\mathrm{~m}, 2 \mathrm{H}), 6.92(\mathrm{~d}, J=8.3 \mathrm{~Hz}, 1 \mathrm{H}), 4.23(\mathrm{dh}, J=16.2,8.8,8.0$ $\mathrm{Hz}, 2 \mathrm{H}), 3.37$ (d, $J=21.3 \mathrm{~Hz}, 2 \mathrm{H}), 1.30(\mathrm{t}, J=7.3 \mathrm{~Hz}, 3 \mathrm{H})$.

${ }^{13} \mathbf{C}$ NMR (126 MHz, $\left.\mathrm{CDCl}_{3}\right) \delta: 152.41,152.34,152.21,134.75,134.59,133.80,133.73$, $133.55,133.49,130.89,130.84,125.41,125.31,121.31,121.29,116.57,115.58,110.46,105.85$, $64.30,64.24,33.44,32.33,16.43,16.38$.

HRMS: $\left[(\mathrm{M}+\mathrm{Na})^{+}\right]$Calcd.: 417.9814; observed: 417.9819 .<smiles>CCOP(=O)(Cc1cc(O)c(F)c(Br)c1)Oc1ccccc1C#N</smiles>

2-cyanophenyl ethyl 3-bromo-4-fluoro-5-hydroxybenzylphosphonate (4g): 
Yield : 65\% (53 mg)

Appearance: Liquid

$\mathbf{R}_{\mathbf{f}}: 0.3$ (50\% ethyl acetate/pet-ether mixture)

Isolation: Petroleum ether :ethyl acetate(v/v) $(1: 1)$

${ }^{1}$ H NMR (500 MHz, Chloroform- $d$ ) $\delta: 7.65(\mathrm{~d}, J=7.7 \mathrm{~Hz}, 1 \mathrm{H}), 7.57(\mathrm{t}, J=8.0 \mathrm{~Hz}, 1 \mathrm{H}), 7.41(\mathrm{~d}$, $J=8.4 \mathrm{~Hz}, 1 \mathrm{H}), 7.32-7.23(\mathrm{~m}, 1 \mathrm{H}), 7.06(\mathrm{~d}, J=7.1 \mathrm{~Hz}, 1 \mathrm{H}), 7.00(\mathrm{dd}, J=5.6,2.7 \mathrm{~Hz}, 1 \mathrm{H})$, $4.28-4.17(\mathrm{~m}, 2 \mathrm{H}), 3.37(\mathrm{~d}, J=21.6 \mathrm{~Hz}, 2 \mathrm{H}), 1.30(\mathrm{t}, J=7.7 \mathrm{~Hz}, 3 \mathrm{H})$.

${ }^{13} \mathrm{C}$ NMR (126 MHz, $\left.\mathrm{CDCl}_{3}\right) \delta: 152.17,152.10,134.74,133.78,126.83,126.75,125.56$, $125.22,125.16,121.26,121.24,118.57,118.53,115.61,114.23,109.64,109.47,105.75,64.75$, $64.69,33.74,32.62,16.38,16.33$.

HRMS: $\left[(\mathrm{M}+\mathrm{Na})^{+}\right]$Calcd.: 435.9720; observed: 435.9722 .

\section{Meta-Acetoxylation:}<smiles>CCOP(=O)(Cc1cccc(OC(C)=O)c1)Oc1ccccc1C#N</smiles>

3-(((2-cyanophenoxy)(ethoxy)phosphoryl)methyl)phenyl acetate (5a):

Yield : 76\% (55 mg)

Appearance: Liquid

$\mathbf{R}_{\mathbf{f}}: 0.3$ (50\% ethyl acetate/pet-ether mixture) 
Isolation: Petroleum ether :ethyl acetate(v/v) $(1: 1)$

${ }^{1}$ H NMR (500 MHz, Chloroform-d) $\delta: 7.58(\mathrm{dd}, \mathrm{J}=7.8,1.7 \mathrm{~Hz}, 1 \mathrm{H}), 7.51-7.45$ (m, 1H), 7.31 $(\mathrm{q}, \mathrm{J}=8.0 \mathrm{~Hz}, 2 \mathrm{H}), 7.25-7.16(\mathrm{~m}, 2 \mathrm{H}), 7.13(\mathrm{q}, \mathrm{J}=2.3 \mathrm{~Hz}, 1 \mathrm{H}), 7.00(\mathrm{dt}, \mathrm{J}=8.2,2.5 \mathrm{~Hz}, 1 \mathrm{H})$, $4.24-4.11(\mathrm{~m}, 2 \mathrm{H}), 3.43(\mathrm{~d}, \mathrm{~J}=22.0 \mathrm{~Hz}, 2 \mathrm{H}), 2.27(\mathrm{~s}, 3 \mathrm{H}), 1.25(\mathrm{t}, \mathrm{J}=7.1 \mathrm{~Hz}, 3 \mathrm{H})$.

${ }^{13} \mathrm{C}$ NMR (126 MHz, $\left.\mathrm{CDCl}_{3}\right) \delta: 169.48,152.34,151.00,150.97,134.53,133.62,131.72$, $131.64,129.87,129.84,127.71,127.65,125.12,123.46,123.40,121.30,121.28,120.91,120.88$, $115.63,105.73,64.14,64.08,34.55,33.45,21.24,16.37,16.32$.

HRMS: $\left[(\mathrm{M}+\mathrm{Na})^{+}\right]$Calcd.: 382.0817; observed: 382.0822 .<smiles>CCOP(=O)(Cc1ccc(C)c(OC(C)=O)c1)Oc1ccccc1C#N</smiles>

5-(((2-cyanophenoxy)(ethoxy)phosphoryl)methyl)-2-methylphenyl acetate (5b):

Yield : $73 \%(54 \mathrm{mg})$

Appearance: Liquid

$\mathbf{R}_{\mathbf{f}}: 0.3$ (50\% ethyl acetate/pet-ether mixture)

Isolation: Petroleum ether :ethyl acetate(v/v) (1:1)

${ }^{1}$ H NMR (400 MHz, Chloroform-d) $\delta: 7.58$ (dt, J = 7.7, $\left.1.2 \mathrm{~Hz}, 1 \mathrm{H}\right), 7.49$ (ddd, J = 8.5, 7.5, 1.8 $\mathrm{Hz}, 1 \mathrm{H}), 7.30$ (dt, J = 8.5, 1.2 Hz, 1H), 7.20 (dt, J = 7.6, $0.9 \mathrm{~Hz}, 1 \mathrm{H}), 7.18-7.15$ (m, 1H), 7.13 $(\mathrm{dt}, \mathrm{J}=7.8,2.1 \mathrm{~Hz}, 1 \mathrm{H}), 7.05(\mathrm{t}, \mathrm{J}=2.2 \mathrm{~Hz}, 1 \mathrm{H}), 4.17(\mathrm{dqd}, \mathrm{J}=8.3,7.1,1.4 \mathrm{~Hz}, 2 \mathrm{H}), 3.39(\mathrm{~d}, \mathrm{~J}=$ $21.7 \mathrm{~Hz}, 2 \mathrm{H}), 2.29$ (s, 3H), $2.14(\mathrm{~d}, \mathrm{~J}=2.5 \mathrm{~Hz}, 3 \mathrm{H}), 1.24$ (t, J = 7.1 Hz, 3H).

${ }^{13} \mathrm{C}$ NMR (101 MHz, $\left.\mathrm{CDCl}_{3}\right) \delta: 169.23,152.47,152.39,149.53,134.53,133.59,131.55$, $131.52,129.56,129.52,128.91,128.81,127.86,127.79,125.03,123.68,123.61,121.34,121.31$, $115.67,105.65,64.09,64.02,34.28,32.89,20.91,16.38,16.32,15.99$.

HRMS: $\left[(\mathrm{M}+\mathrm{Na})^{+}\right]$Calcd.: 396.0971; observed: 396.0977.<smiles>CCOP(=O)(Cc1ccc(OC)c(OC(C)=O)c1)Oc1ccccc1C#N</smiles>

\section{5-(((2-cyanophenoxy)(ethoxy)phosphoryl)methyl)-2-methoxyphenyl acetate (5c):}

Yield : 74\% (58 mg)

Appearance: Liquid

$\mathbf{R}_{\mathbf{f}}: 0.3$ (50\% ethyl acetate/pet-ether mixture) 
Isolation: Petroleum ether : ethyl acetate(v/v) $(1: 1)$

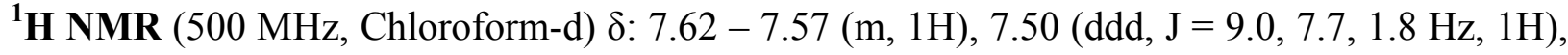
$7.29(\mathrm{~d}, \mathrm{~J}=8.5 \mathrm{~Hz}, 1 \mathrm{H}), 7.23-7.18(\mathrm{~m}, 2 \mathrm{H}), 7.08(\mathrm{t}, \mathrm{J}=2.4 \mathrm{~Hz}, 1 \mathrm{H}), 6.91(\mathrm{~d}, \mathrm{~J}=8.4 \mathrm{~Hz}, 1 \mathrm{H})$, 4.18 (qdt, J = 7.2, 4.7, 2.6 Hz, 2H), $3.81(\mathrm{~s}, 3 \mathrm{H}), 3.37$ (d, J = 21.4 Hz, 2H), 2.29 (s, 3H), $1.26(\mathrm{t}, \mathrm{J}$ $=7.0 \mathrm{~Hz}, 3 \mathrm{H})$.

${ }^{13} \mathrm{C}$ NMR (126 MHz, $\left.\mathrm{CDCl}_{3}\right) \delta: 169.10,152.54,152.47,150.73,139.94,139.91,134.61$, $133.64,128.67,128.61,125.10,124.69,124.64,122.33,122.25,121.38,121.36,115.72,112.83$, $112.80,105.75,64.14,64.08,56.12,33.76,32.64,20.82,16.44,16.39$.

HRMS: $\left[(\mathrm{M}+\mathrm{Na})^{+}\right]$Calcd.: 412.0919; observed: 412.0922.<smiles>CCOP(=O)(Cc1ccc(Br)c(OC(C)=O)c1)Oc1ccccc1C#N</smiles>

\section{2-bromo-5-(((2-cyanophenoxy)(ethoxy)phosphoryl)methyl)phenyl acetate (5d):}

Yield : 64\% (55 mg)

Appearance: Liquid

$\mathbf{R}_{\mathbf{f}}: 0.3$ (50\% ethyl acetate/pet-ether mixture)

Isolation: Petroleum ether :ethyl acetate(v/v) $(1: 1)$

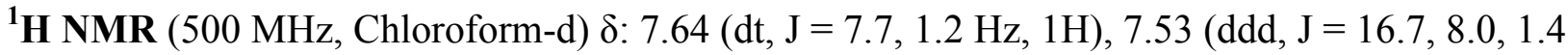
$\mathrm{Hz}, 2 \mathrm{H}), 7.34(\mathrm{dd}, \mathrm{J}=8.7,1.3 \mathrm{~Hz}, 1 \mathrm{H}), 7.22$ (t, J = $7.7 \mathrm{~Hz}, 1 \mathrm{H}), 7.19$ (t, J = 2.5 Hz, 1H), 7.14 $(\mathrm{dt}, \mathrm{J}=8.3,2.5 \mathrm{~Hz}, 1 \mathrm{H}), 4.25-4.18(\mathrm{~m}, 2 \mathrm{H}), 3.40(\mathrm{~d}, \mathrm{~J}=22.0 \mathrm{~Hz}, 2 \mathrm{H}), 2.36(\mathrm{~s}, 3 \mathrm{H}), 1.27(\mathrm{t}, \mathrm{J}=$ $7.1 \mathrm{~Hz}, 3 \mathrm{H})$.

${ }^{13} \mathbf{C}$ NMR (126 MHz, $\left.\mathrm{CDCl}_{3}\right) \delta: 168.56,152.35,152.28,148.59,134.65,133.71,133.67$, $131.20,131.13,129.19,129.14,125.58,125.53,125.29,121.36,121.34,115.66,105.79,64.34$, $64.28,34.24,33.12,20.94,16.42,16.38$.

HRMS: $\left[(\mathrm{M}+\mathrm{Na})^{+}\right]$Calcd.: 459.9921; observed: 459.9917.<smiles>CCOP(=O)(Cc1ccc(Cl)c(OC(C)=O)c1)Oc1ccccc1C#N</smiles>

2-chloro-5-(((2-cyanophenoxy)(ethoxy)phosphoryl)methyl)phenyl acetate (5e):

Yield : 67\% (53 mg)

Appearance: Liquid

$\mathbf{R}_{\mathbf{f}}: 0.3$ (50\% ethyl acetate/pet-ether mixture) 
Isolation: Petroleum ether :ethyl acetate(v/v) (1:1)

${ }^{1}$ H NMR (500 MHz, Chloroform-d) $\delta: 7.61$ (dt, J = 7.7, $1.2 \mathrm{~Hz}, 1 \mathrm{H}$ ), 7.52 (ddd, J = 8.5, 7.5, 1.7 $\mathrm{Hz}, 1 \mathrm{H}), 7.40-7.37(\mathrm{~m}, 1 \mathrm{H}), 7.33(\mathrm{dt}, \mathrm{J}=8.4,1.2 \mathrm{~Hz}, 1 \mathrm{H}), 7.23(\mathrm{dt}, \mathrm{J}=7.7,0.9 \mathrm{~Hz}, 1 \mathrm{H}), 7.20$ $(\mathrm{dt}, \mathrm{J}=4.7,2.2 \mathrm{~Hz}, 2 \mathrm{H}), 4.19(\mathrm{dqd}, \mathrm{J}=8.3,7.1,4.3 \mathrm{~Hz}, 2 \mathrm{H}), 3.41(\mathrm{dd}, \mathrm{J}=22.1,1.0 \mathrm{~Hz}, 2 \mathrm{H})$, $2.34(\mathrm{~s}, 3 \mathrm{H}), 1.26(\mathrm{t}, \mathrm{J}=7.0 \mathrm{~Hz}, 3 \mathrm{H})$.

${ }^{13}$ C NMR $\left(126 \mathrm{MHz}, \mathrm{CDCl}_{3}\right) \delta: 168.52,152.28,147.27,134.66,134.52,133.72,130.67$, $130.64,130.40,130.32,128.86,128.81,125.54,125.49,125.31,121.37,121.34,120.64,116.57$, $105.81,64.35,64.29,34.20,33.09,20.77,16.43,16.38$.

HRMS: $\left[(\mathrm{M}+\mathrm{Na})^{+}\right]$Calcd.: 416.0425 ; observed: 416.0429 .<smiles>CCOP(=O)(Cc1cc(Br)c(F)c(OC(C)=O)c1)Oc1ccccc1C#N</smiles>

3-bromo-5-(((2-cyanophenoxy)(ethoxy)phosphoryl)methyl)-2-fluorophenyl acetate (5f):

Yield : 59\% (53 mg)

Appearance: Liquid

$\mathbf{R}_{\mathbf{f}}: 0.3$ (50\% ethyl acetate/pet-ether mixture)

Isolation: Petroleum ether :ethyl acetate(v/v) (1:1)

${ }^{1}$ H NMR (500 MHz, Chloroform- $d$ ) $\delta: 7.61$ (dt, $J=7.7,1.3 \mathrm{~Hz}, 1 \mathrm{H}$ ), 7.53 (dddd, $J=9.4,5.2$, $3.5,1.7 \mathrm{~Hz}, 1 \mathrm{H}), 7.41(\mathrm{qd}, J=4.8,4.3,2.0 \mathrm{~Hz}, 1 \mathrm{H}), 7.37(\mathrm{dt}, J=8.5,1.2 \mathrm{~Hz}, 1 \mathrm{H}), 7.23(\mathrm{tt}, J=$ 7.6, $0.9 \mathrm{~Hz}, 1 \mathrm{H}), 7.16$ (dt, $J=6.4,2.5 \mathrm{~Hz}, 1 \mathrm{H}), 4.24-4.16(\mathrm{~m}, 2 \mathrm{H}), 3.36$ (d, $J=21.9 \mathrm{~Hz}, 2 \mathrm{H})$, $2.32(\mathrm{~s}, 3 \mathrm{H}), 1.27(\mathrm{t}, \mathrm{J}=7.3 \mathrm{~Hz}, 3 \mathrm{H})$.

${ }^{13} \mathbf{C}$ NMR $\left(126 \mathrm{MHz}, \mathrm{CDCl}_{3}\right) \delta: 168.00,152.27,152.21,150.02,149.98,138.97,138.83$, $134.64,133.72,132.04,131.99,127.66,127.62,127.58,127.54,125.38,124.85,124.80,121.25$, $115.58,110.35,110.16,105.73,64.35,64.29,33.74,32.62,20.59,16.42,16.37$.

${ }^{31} \mathbf{P}$ NMR $\left(202 \mathrm{MHz}, \mathrm{CDCl}_{3}\right) \delta: 22.52$

HRMS: $\left[(\mathrm{M}+\mathrm{Na})^{+}\right]$Calcd.: 477.9829; observed: 477.9834 .

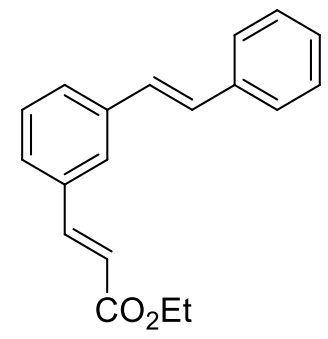

(E)-ethyl 3-(3-styrylphenyl)39crylates (6a): 
Yield: 75\% (41 mg, $0.2 \mathrm{mmol} \mathrm{scale).}$

Appearance: Solid

$\mathbf{R}_{\mathbf{f}}: 0.5$ (5\% ethyl acetate/pet-ether mixture)

Isolation: Petroleum ether: ethyl acetate (v/v) (99:1)

${ }^{1}$ H NMR (500 MHz, Chloroform- $d$ ) $\delta: 7.74(\mathrm{dd}, J=16.0,1.8 \mathrm{~Hz}, 1 \mathrm{H}), 7.68(\mathrm{t}, J=1.8 \mathrm{~Hz}, 1 \mathrm{H})$, $7.58-7.53(\mathrm{~m}, 3 \mathrm{H}), 7.45$ (dt, $J=7.7,1.6 \mathrm{~Hz}, 1 \mathrm{H}), 7.40(\mathrm{dd}, J=8.6,6.8 \mathrm{~Hz}, 3 \mathrm{H}), 7.34-7.27$

$(\mathrm{m}, 1 \mathrm{H}), 7.15(\mathrm{dd}, J=8.9,1.8 \mathrm{~Hz}, 2 \mathrm{H}), 6.52(\mathrm{dd}, J=16.0,1.7 \mathrm{~Hz}, 1 \mathrm{H}), 4.31$ (qd, $J=7.1,1.8 \mathrm{~Hz}$, $2 \mathrm{H}), 1.38(\mathrm{td}, J=7.1,1.8 \mathrm{~Hz}, 3 \mathrm{H})$.

${ }^{13}$ C NMR (126 MHz, $\left.\mathrm{CDCl}_{3}\right) \delta: 14.4,60.6,118.6,126.1,126.6,127.1,127.8,127.9,128.2$, $128.8,129.2,129.7,134.9,137.0,138.0,144.5,167.0$.

HRMS: [(M+Na) $\left.{ }^{+}\right]$calcd: 301.1199 ; observed: 301.1199.<smiles>CC(=O)Oc1cccc(/C=C/c2ccccc2)c1</smiles>

(E)-3-styrylphenyl acetate (6b):

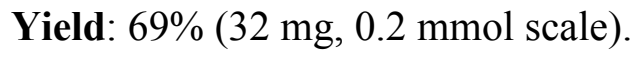

Appearance: Solid

$\mathbf{R}_{\mathbf{f}}: 0.5$ (10\% ethyl acetate/pet-ether mixture)

Isolation: Petroleum ether: ethyl acetate (v/v) (95:5)

${ }^{1}$ H NMR (400 MHz, Chloroform-d) $\delta: ~ 7.56-7.51$ (m, 2H), 7.42 - 7.37 (m, 4H), 7.33 - 7.27 (m, $2 \mathrm{H}), 7.12(\mathrm{~d}, \mathrm{~J}=3.0 \mathrm{~Hz}, 2 \mathrm{H}), 7.05-7.00(\mathrm{~m}, 1 \mathrm{H}), 2.34(\mathrm{~s}, 3 \mathrm{H})$.

${ }^{13} \mathbf{C}$ NMR (101 MHz, $\left.\mathrm{CDCl}_{3}\right) \delta: 169.58,151.17,139.15,137.06,129.86,129.70,128.82$, $127.99,127.77,126.72,124.30,120.75,119.35,21.27$.

HRMS: [(M+Na) $\left.{ }^{+}\right]$Calcd.: 261.0888; observed: 261.0893 .<smiles>Oc1cccc(/C=C/c2ccccc2)c1</smiles>

\section{(E)-3-styrylphenol (6c):}

Yield: 64\% (26 mg, $0.2 \mathrm{mmol}$ scale).

Appearance: Solid

$\mathbf{R}_{\mathbf{f}}: 0.5$ (10\% ethyl acetate/pet-ether mixture)

Isolation: Petroleum ether: ethyl acetate (v/v) (95:5) 
${ }^{1}$ H NMR (400 MHz, Chloroform-d) $\delta: 7.54-7.48(\mathrm{~m}, 2 \mathrm{H}), 7.41-7.33(\mathrm{~m}, 2 \mathrm{H}), 7.31-7.21$ (m, 2H), $7.13-7.05(\mathrm{~m}, 3 \mathrm{H}), 7.00(\mathrm{t}, \mathrm{J}=1.9 \mathrm{~Hz}, 1 \mathrm{H}), 6.75(\mathrm{ddd}, \mathrm{J}=8.0,2.5,1.0 \mathrm{~Hz}, 1 \mathrm{H})$.

${ }^{13} \mathbf{C}$ NMR (101 $\left.\mathrm{MHz}, \mathrm{CDCl}_{3}\right) \delta: 155.94,139.29,137.32,130.06,129.39,128.87,128.40$, 127.93, 126.74, 119.67, 114.85, 113.13 .

HRMS: Calcd.: 219.0783; observed: 219.0783.

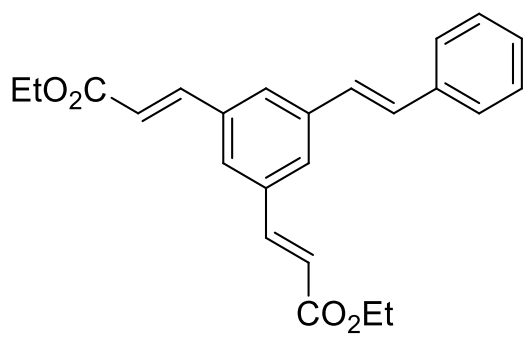

(2E,2'E)-diethyl 3,3'-(5-styryl-1,3-phenylene)diacrylate (6d):

Yield: $68 \%$ (50 mg, $0.2 \mathrm{mmol}$ scale).

Appearance: Solid

$\mathbf{R}_{\mathbf{f}}: 0.5$ (10\% ethyl acetate/pet-ether mixture)

Isolation: Petroleum ether: ethyl acetate (v/v) (96:4)

${ }^{1}$ H NMR (400 MHz, Chloroform- $d$ ) $\delta: 7.70(\mathrm{~d}, J=16.0 \mathrm{~Hz}, 2 \mathrm{H}), 7.64(\mathrm{~d}, J=1.5 \mathrm{~Hz}, 2 \mathrm{H}), 7.56$ - $7.50(\mathrm{~m}, 3 \mathrm{H}), 7.39(\mathrm{dd}, J=8.4,6.8 \mathrm{~Hz}, 2 \mathrm{H}), 7.33-7.27(\mathrm{~m}, 1 \mathrm{H}), 7.17(\mathrm{~d}, J=16.4 \mathrm{~Hz}, 1 \mathrm{H})$, $7.08(\mathrm{~d}, J=16.3 \mathrm{~Hz}, 1 \mathrm{H}), 6.51(\mathrm{~d}, J=16.0 \mathrm{~Hz}, 2 \mathrm{H}), 4.29$ (q, $J=7.1 \mathrm{~Hz}, 4 \mathrm{H}), 1.36(\mathrm{t}, J=7.1 \mathrm{~Hz}$, $6 \mathrm{H})$.

${ }^{13}$ C NMR (101 MHz, $\left.\mathrm{CDCl}_{3}\right) \delta: 14.4,60.8,119.6,126.5,126.8,127.1,127.5,128.3,129.0$, 130.6, 135.6, 136.7, 138.8, 143.7, 166.8.

HRMS: $\left[(\mathrm{M}+\mathrm{Na})^{+}\right]$calcd: 399.1570; observed: 399.1568.

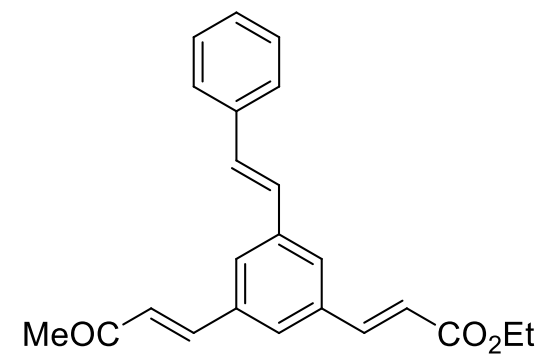

(E)-ethyl 3-(3-((E)-3-oxobut-1-enyl)-5-styrylphenyl)41crylates (6e):

Yield: $71 \%$ (49 mg, $0.2 \mathrm{mmol} \mathrm{scale})$.

Appearance: Solid

$\mathbf{R}_{\mathbf{f}}: 0.5$ (5\% ethyl acetate/pet-ether mixture)

Isolation: Petroleum ether: ethyl acetate (v/v) $(9: 1)$

${ }^{1}$ H NMR (500 MHz, Chloroform-d) $\delta: 7.70(\mathrm{~d}, J=16.1 \mathrm{~Hz}, 1 \mathrm{H}), 7.67(\mathrm{~d}, J=1.7 \mathrm{~Hz}, 2 \mathrm{H}), 7.57$ $-7.50(\mathrm{~m}, 4 \mathrm{H}), 7.39$ (t, J=7.5 Hz, 2H), $7.34-7.28(\mathrm{~m}, 1 \mathrm{H}), 7.18(\mathrm{~d}, J=16.3 \mathrm{~Hz}, 1 \mathrm{H}), 7.09$ (d, 
$J=16.3 \mathrm{~Hz}, 1 \mathrm{H}), 6.79(\mathrm{~d}, J=16.2 \mathrm{~Hz}, 1 \mathrm{H}), 6.52(\mathrm{~d}, J=16.0 \mathrm{~Hz}, 1 \mathrm{H}), 4.29$ (q, $J=7.1 \mathrm{~Hz}, 2 \mathrm{H})$, $2.41(\mathrm{~s}, 3 \mathrm{H}), 1.36(\mathrm{t}, J=7.1 \mathrm{~Hz}, 3 \mathrm{H})$.

${ }^{13}$ C NMR $\left(126 \mathrm{MHz}, \mathrm{CDCl}_{3}\right) \delta: 14.3,27.8,60.7,119.6,126.6,126.7,126.9,127.5,127.6$, 128.0, 128.3, 128.8, 130.7, 135.5, 135.6, 136.6, 138.8, 142.3, 143.5, 166.7, 198.1 .

HRMS: [(M+Na) $\left.{ }^{+}\right]$calcd: 369.1466 ; observed: 369.1468

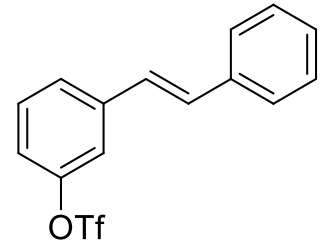

(E)-3-styrylphenyl trifluoromethanesulfonate (7):

Yield: $85 \%$ (279 mg, $1 \mathrm{mmol} \mathrm{scale})$.

Appearance: liquid

$\mathbf{R}_{\mathbf{f}}: 0.5$ (5\% ethyl acetate/pet-ether mixture)

Isolation: Petroleum ether: ethyl acetate (v/v) (97:3)

${ }^{1}$ H NMR (400 MHz, Chloroform-d) $\delta: 7.52$ (ddt, J = 7.1, 6.0, $\left.1.4 \mathrm{~Hz}, 3 \mathrm{H}\right), 7.46-7.35$ (m, 4H), $7.35-7.28(\mathrm{~m}, 1 \mathrm{H}), 7.18-7.10(\mathrm{~m}, 2 \mathrm{H}), 7.07$ (d, J = $16.4 \mathrm{~Hz}, 1 \mathrm{H})$.

${ }^{13} \mathbf{C}$ NMR $\left(101 \mathrm{MHz}, \mathrm{CDCl}_{3}\right) \delta: 151.09,140.38,136.57,131.44,130.55,128.99,128.56$, $126.96,126.64,126.51,120.05,119.07$.

HRMS: Calcd.: 351.0277; observed: 351.0275 .

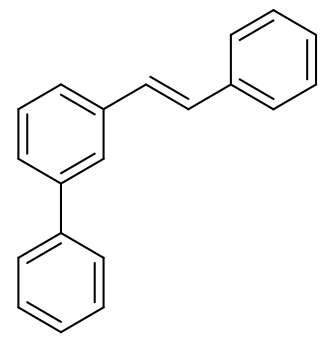

(E)-3-styryl-1,1'-biphenyl (7a):

Yield: $75 \%$ ( $38 \mathrm{mg}, 0.2 \mathrm{mmol}$ scale).

Appearance: Solid

$\mathbf{R}_{\mathbf{f}}: 0.5$ (pet-ether)

Isolation: Petroleum ether

${ }^{1} \mathbf{H}$ NMR $(500 \mathrm{MHz}$, Chloroform-d) $\delta 7.74(\mathrm{t}, \mathrm{J}=1.8 \mathrm{~Hz}, 1 \mathrm{H}), 7.68-7.62(\mathrm{~m}, 2 \mathrm{H}), 7.59-7.54$ $(\mathrm{m}, 2 \mathrm{H}), 7.54-7.43(\mathrm{~m}, 5 \mathrm{H}), 7.39(\mathrm{t}, \mathrm{J}=7.6 \mathrm{~Hz}, 3 \mathrm{H}), 7.32-7.26(\mathrm{~m}, 1 \mathrm{H}), 7.20(\mathrm{~s}, 2 \mathrm{H})$.

${ }^{13} \mathbf{C}$ NMR $\left(126 \mathrm{MHz}, \mathrm{CDCl}_{3}\right) \delta: 141.89,141.28,137.96,137.43,129.27,129.22,128.96$, $128.89,128.76,127.88,127.58,127.39,126.73,126.70,125.60,125.55$. 
HRMS: Calcd.: 279.1148; observed: 279.1151.<smiles>C(=C/c1ccccc1)\c1ccccc1</smiles>

(E)-N-phenyl-3-styrylaniline (7b):

Yield: $83 \%$ (45mg, $0.2 \mathrm{mmol}$ scale).

Appearance: Solid

$\mathbf{R}_{\mathbf{f}}: 0.5$ (10\% ethyl acetate/pet-ether mixture)

Isolation: Petroleum ether: ethyl acetate (v/v) (95:5)

${ }^{1}$ H NMR (400 MHz, Chloroform-d) $\delta 7.55-7.47$ (m, 3H), 7.37 (t, J = 7.6 Hz, 3H), $7.31-7.26$ $(\mathrm{m}, 1 \mathrm{H}), 7.26-7.20(\mathrm{~m}, 2 \mathrm{H}), 7.18(\mathrm{~d}, \mathrm{~J}=7.9 \mathrm{~Hz}, 1 \mathrm{H}), 7.13-7.03(\mathrm{~m}, 4 \mathrm{H}), 6.99(\mathrm{t}, \mathrm{J}=2.0 \mathrm{~Hz}$, $1 \mathrm{H}), 6.74(\mathrm{dt}, \mathrm{J}=8.1,2.6 \mathrm{~Hz}, 3 \mathrm{H})$.

${ }^{13} \mathrm{C}$ NMR (101 MHz, $\left.\mathrm{CDCl}_{3}\right) \delta: 156.02,139.23,137.32,130.03,129.51,129.32,128.86$, $128.43,127.90,126.73,119.57,119.24,115.67,114.88,113.14$.

HRMS: Calcd.: 294.1256; observed: 294.1258.

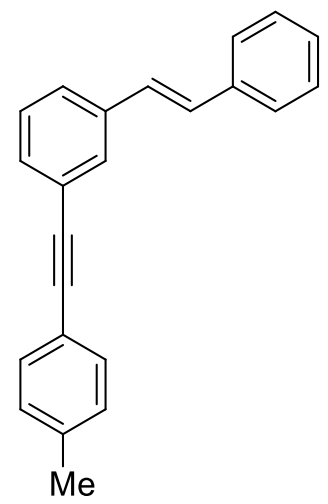

(E)-1-styryl-3-(p-tolylethynyl)benzene (7c):

Yield: 94\% (55mg, $0.2 \mathrm{mmol}$ scale).

Appearance: Solid

$\mathbf{R}_{\mathbf{f}}: 0.5$ (pet-ether)

Isolation: Petroleum ether

${ }^{1}$ H NMR (500 MHz, Chloroform-d) $\delta 7.71(\mathrm{~d}, \mathrm{~J}=1.8 \mathrm{~Hz}, 1 \mathrm{H}), 7.57-7.51(\mathrm{~m}, 2 \mathrm{H}), 7.47$ (dd, J $=8.3,2.0 \mathrm{~Hz}, 3 \mathrm{H}), 7.43(\mathrm{dt}, \mathrm{J}=7.7,1.4 \mathrm{~Hz}, 1 \mathrm{H}), 7.37(\mathrm{dt}, \mathrm{J}=19.5,7.6 \mathrm{~Hz}, 3 \mathrm{H}), 7.32-7.25(\mathrm{~m}$, $1 \mathrm{H}), 7.22-7.06(\mathrm{~m}, 4 \mathrm{H}), 2.39(\mathrm{~s}, 3 \mathrm{H})$. 
${ }^{13} \mathbf{C}$ NMR $\left(126 \mathrm{MHz}, \mathrm{CDCl}_{3}\right) \delta: 138.65,137.66,137.25,131.70,130.75,129.63,129.62$, $129.32,128.89,128.85,126.76,126.50,124.02,89.85,88.79,21.71$.

HRMS: Calcd.: 317.1304; observed: 317.1308 .

\section{${ }^{1}$ H NMR Study:}

$\mathrm{Pd}(\mathrm{Oac})_{2}(0.05 \mathrm{mmol}, 11 \mathrm{mg})$, Ac-Phe-OH $(0.1 \mathrm{mmol}, 21 \mathrm{mg})$, substrate $1 \mathrm{a}(0.5 \mathrm{mmol}, 15 \mathrm{mg})$, HFIP $(25 \mu l)$ were taken in a NMR tube and then recorded the ${ }^{1} \mathrm{H}$ NMR spectrum.

Out of two different modes (linear and side-on) of nitrile bindings, linear coordination was confirmed from downfield shift of ortho and para proton of 2-cyano phenol core in ${ }^{1} \mathrm{H}$ spectra of substrate 1a.

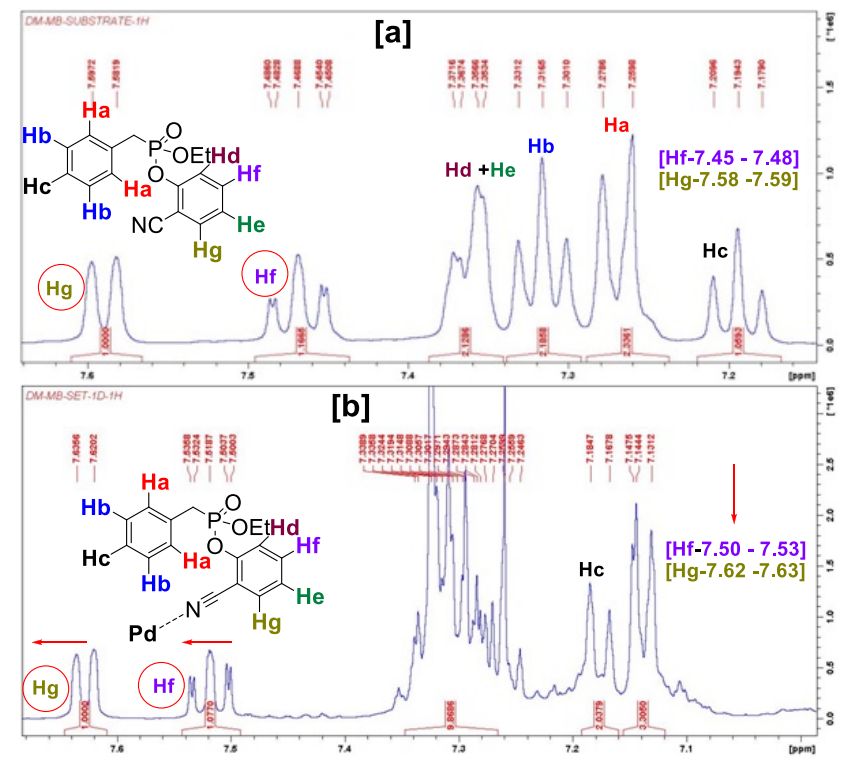

Figure S2: ${ }^{1} \mathrm{H}$ NMR Study

\section{ESI-MS study:}

$\mathrm{Pd}(\mathrm{Oac})_{2}(0.05 \mathrm{mmol}, 11 \mathrm{mg})$, Ac-Phe-OH $(0.1 \mathrm{mmol}, 21 \mathrm{mg})$, substrate $1 \mathrm{a}(0.5 \mathrm{mmol}, 15 \mathrm{mg})$, HFIP $(25 \mu \mathrm{l})$ were mixed within a sample vial then stirred for $1 \mathrm{~h}$. After that ESI-MS spectrum was recorded.

In mass spectrum, a metal-ligand-substrate adduct such as [(Ac-Phe-OH) $\left.\mathrm{Pd}^{\mathrm{II}}\left(\mathbf{1} \mathbf{a}-\mathrm{H}^{+}\right)\right]$was appeared as a possible intermediate species with desired isotopic pattern. 


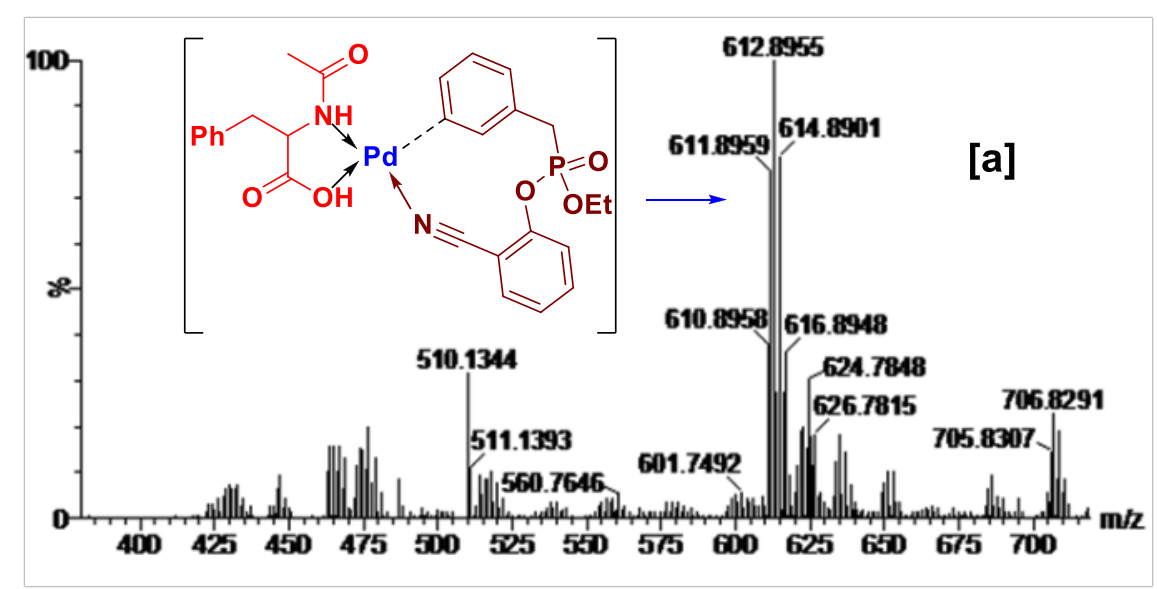

Figure S3: ESI-MS study

In order to further confirm the formation of macrocyclic intermediate, 1a was replaced with 4methyl substituted phosphonic ester (1b), that also exhibits ligand free intermediate formation such as $\left[\mathrm{Pd}^{\mathrm{II}}\left(\mathbf{1} \mathbf{b}-\mathrm{H}^{+}\right)\right]$.

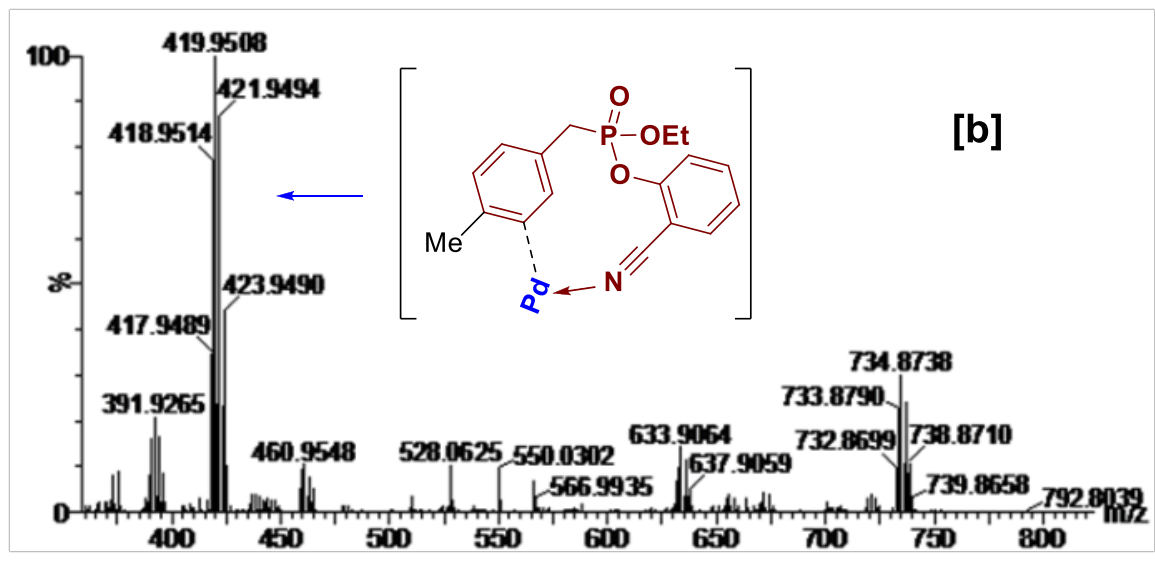

Figure S4: ESI-MS study

\section{Intermolecular competition experiment:}

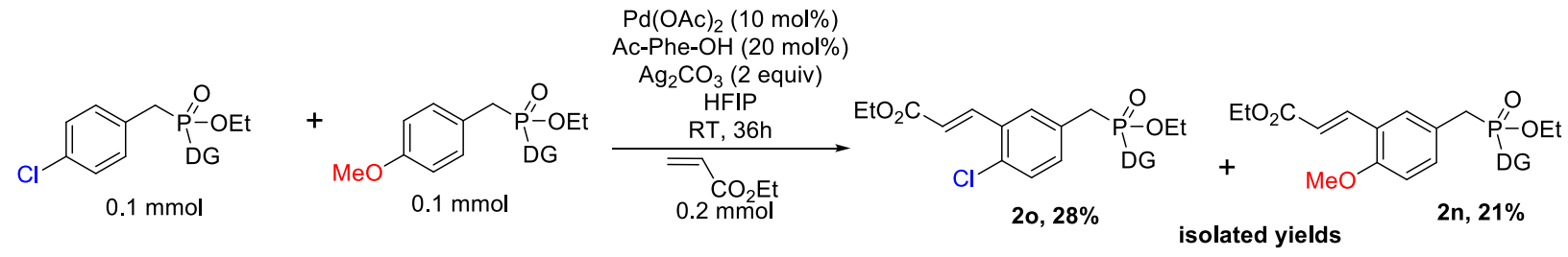

Scheme S14: Intermolecular competition experiment

An oven-dried screw cap reaction tube was charged with a magnetic stir-bar, $\mathrm{Pd}(\mathrm{OAc})_{2}(10$ mol\%, $5 \mathrm{mg}$ ), Ac-Phe-OH (20 mol\%, $8 \mathrm{mg}), \mathrm{Ag}_{2} \mathrm{CO}_{3}$ (2 equiv.; $110 \mathrm{mg}$ ), 4-chloro benzylphosphonate ester $(0.1 \mathrm{mmol}, 34 \mathrm{mg})$ and 4-methoxy benzylphosphonate $(0.1 \mathrm{mmol}, 33$ 
$\mathrm{mg})$ and ethyl acrylate $(0.2 \mathrm{mmol})$. Solid reagents were weighed first followed by liquid reagents. $1.5 \mathrm{~mL}$ of 1,1,1,3,3,3-Hexafluoro-2-propanol (HFIP) were added. The reaction mixture was stirred vigorously at room temperature for $36 \mathrm{~h}$. The reaction was taken out after 36 $\mathrm{h}$ and the reaction mixture was diluted with EtOAc and filtered through a celite pad. After filtration and evaporation of the solvent, the crude mixture was purified by column chromatography using silica gel (100-200 mesh size) and petroleum ether/ ethyl acetate as the eluent (13:7). Compound 2o was isolated in $12 \mathrm{mg}$, and compound $2 \mathrm{n}$ was isolated in $9 \mathrm{mg}$.

\section{Intermolecular KIE experiment:}

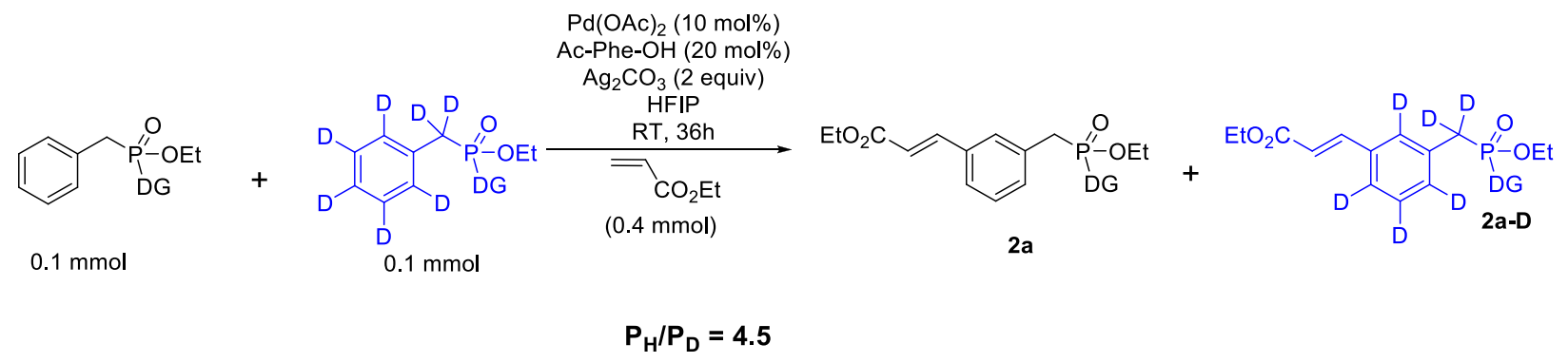

Scheme S15: $\mathrm{P}_{\mathrm{H}} / \mathrm{P}_{\mathrm{D}}$ experiment

An oven-dried screw cap reaction tube was charged with a magnetic stir-bar, $\mathrm{Pd}(\mathrm{OAc})_{2}(10$ mol\%, $5 \mathrm{mg}$ ), Ac-Phe-OH (20 mol\%, $8 \mathrm{mg}$ ), $\mathrm{Ag}_{2} \mathrm{CO}_{3}$ (2 equiv.; $110 \mathrm{mg}$ ), benzylphosphonate ester 1a $(0.1 \mathrm{mmol}, 30 \mathrm{mg})$ and $\left[\mathrm{D}_{7}\right]$ benzylphosphonate $(0.1 \mathrm{mmol}, 31 \mathrm{mg})$ and ethyl acrylate $(0.4 \mathrm{mmol})$. Solid reagents were weighed first followed by liquid reagents. $1.5 \mathrm{~mL}$ of 1,1,1,3,3,3-Hexafluoro-2-propanol (HFIP) were added. The reaction mixture was stirred vigorously at room temperature for $36 \mathrm{~h}$. The reaction was taken out after $36 \mathrm{~h}$ and the reaction mixture was diluted with EtOAc and filtered through a celite pad. After filtration and evaporation of the solvent, the crude mixture was purified by column chromatography using silica gel (100-200 mesh size) and petroleum ether/ ethyl acetate as the eluent (13:7). After isolation, $\mathrm{P}_{\mathrm{H}} / \mathrm{P}_{\mathrm{D}}$ was calculated from $1 \mathrm{H}$ NMR spectrum of the isolated product. From NMR spectrum product distribution $\mathrm{P}_{\mathrm{H}} / \mathrm{P}_{\mathrm{D}}$ was found 4.5 .<smiles>[2H]c1c([2H])c([2H])c(C([2H])([2H])P(=O)(OCC)Oc2ccccc2C#N)c([2H])c1[2H]</smiles>

\section{$\left[\mathrm{D}_{7}\right]$ Benzylphosphonate ester (1a-D):}

${ }^{1} \mathbf{H}$ NMR $\left(400 \mathrm{MHz}, \mathrm{CDCl}_{3}\right) \delta 7.63(\mathrm{~d}, J=7.8 \mathrm{~Hz}, 1 \mathrm{H}), 7.50-7.43(\mathrm{~m}, 1 \mathrm{H}), 7.24(\mathrm{~d}, J=7.8 \mathrm{~Hz}$, $1 \mathrm{H}), 7.19(\mathrm{t}, J=7.6 \mathrm{~Hz}, 1 \mathrm{H}), 4.21-4.11(\mathrm{~m}, 2 \mathrm{H}), 1.24(\mathrm{t}, J=7.1 \mathrm{~Hz}, 3 \mathrm{H})$.

${ }^{13}$ C NMR (126 MHz, $\left.\mathrm{CDCl}_{3}\right) \delta 152.27,134.56,134.40,133.65,133.55,129.68(\mathrm{~m}), 128.33(\mathrm{~m})$, 126.97 (t), 125.31, 125.01, 121.18, 121.16, 120.73, 120.71, 115.27, 105.67, 65.63, 65.58, 34.00 (m), $32.95(\mathrm{~m}), 16.13,16.08$. 
HRMS: [M+ $\mathrm{Na}^{+}$] Calcd.: 331.1199; observed: 331.1193.<smiles>CCOCC=Cc1cc(CP(=O)(OCC)Oc2ccccc2C#N)ccc1C(C)(C)C</smiles>

Isolated yield: $15 \%(10 \mathrm{mg}, 0.15 \mathrm{mmol}$ scale $)$

Appearance: Liquid

$\mathbf{R}_{\mathbf{f}}: 0.3$ (30\% ethyl acetate/pet-ether mixture)

Isolation: Petroleum ether :ethyl acetate(v/v) (13:7)

(E)-ethyl3-(2-tert-butyl-5-(((2-cyanophenoxy)(ethoxy)phosphoryl)methyl)phenyl)acrylates (20):

${ }^{1}$ H NMR $\left(400 \mathrm{MHz}, \mathrm{CDCl}_{3}\right) \delta 8.04(\mathrm{~d}, J=15.7 \mathrm{~Hz}, 1 \mathrm{H}), 7.61-7.55(\mathrm{~m}, 3 \mathrm{H}), 7.45(\mathrm{ddd}, J=$ 8.5, 7.6, $1.7 \mathrm{~Hz}, 1 \mathrm{H}), 7.37$ (dd, $J=4.1,2.1 \mathrm{~Hz}, 2 \mathrm{H}), 7.19(\mathrm{dd}, J=11.1,4.1 \mathrm{~Hz}, 1 \mathrm{H}), 6.34(\mathrm{~d}, J=$ $15.7 \mathrm{~Hz}, 1 \mathrm{H}), 4.35-4.16(\mathrm{~m}, 4 \mathrm{H}), 3.56(\mathrm{~d}, J=22.1 \mathrm{~Hz}, 2 \mathrm{H}), 1.39$ (td, $J=7.1,1.1 \mathrm{~Hz}, 3 \mathrm{H}), 1.34$ (t, $J=7.1 \mathrm{~Hz}, 3 \mathrm{H}), 1.31(\mathrm{~s}, 9 \mathrm{H})$.

${ }^{13}$ C NMR (101 MHz, $\left.\mathrm{CDCl}_{3}\right) \delta 166.86,152.59,152.47,142.34,142.31,134.63,134.41,133.99$, $133.92,133.74,133.67,131.84,131.78,129.32$, 127.84, 127.80, 126.75, 126.65, 125.34, 124.99, $124.14,124.10,120.99,120.96,120.65,115.57,105.75,64.04,63.96,60.78,31.75,31.35$, $30.37,16.37,16.30,14.51$. 


\section{Substrate NMR:}

2-cyanophenyl ethyl benzylphosphonate (1a):

2-cyanophenyl ethyl benzylphosphonate

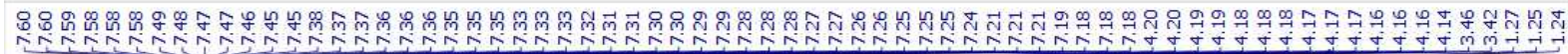
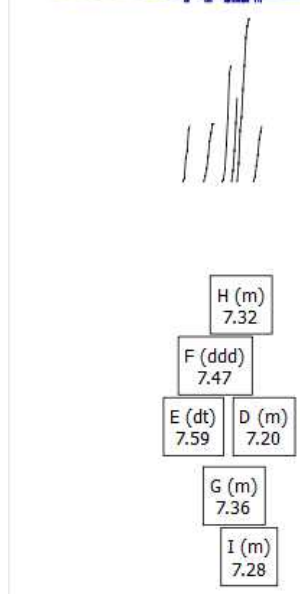<smiles>CCOP(=O)(Cc1ccccc1)Oc1ccccc1C#N</smiles>
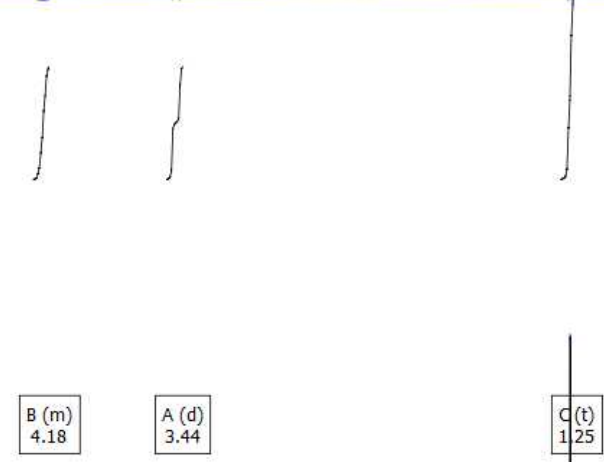

.44
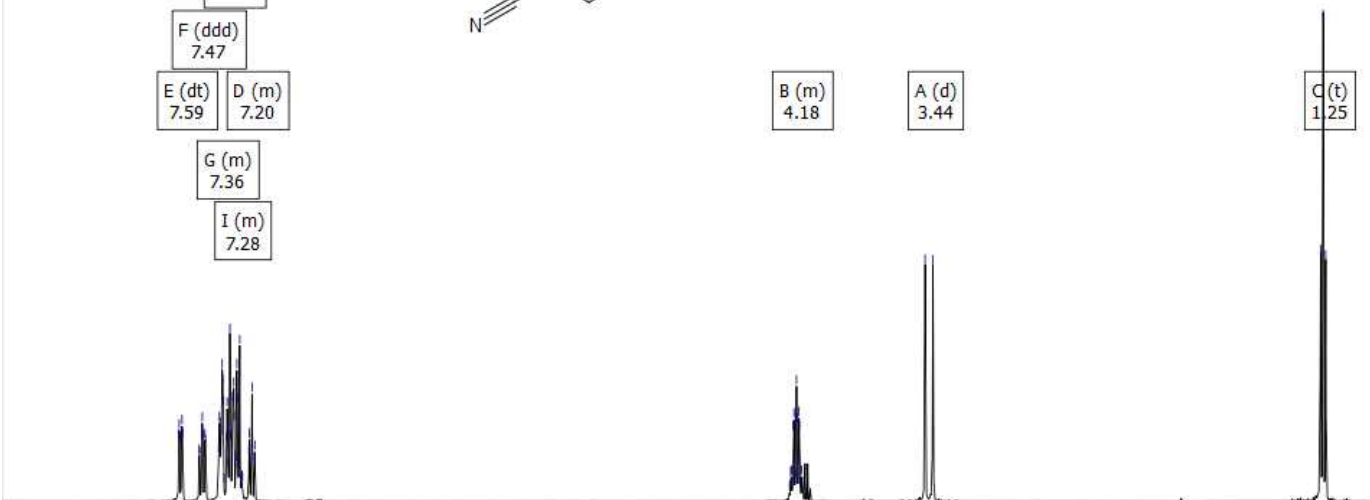

\begin{tabular}{|c|c|c|c|c|c|c|c|c|c|c|c|c|c|c|c|c|}
\hline & & 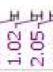 & & & & & & $\begin{array}{l}T \\
8 \\
8 \\
\text { Ni }\end{array}$ & $\begin{array}{l}\text { T' } \\
\stackrel{\text { d }}{\mathrm{N}}\end{array}$ & & & & & & & \\
\hline 8.5 & 8.0 & 7.5 & 7.0 & 6.5 & 6.0 & 5.5 & 5.0 & ${ }^{4.5} \mathrm{f1}(\mathrm{ppm})^{4.0}$ & 3.5 & 3.0 & 2.5 & 2.0 & 1.5 & 1.0 & 0.5 & 0.0 \\
\hline
\end{tabular}

2-cyanophenyl ethyl benzylphosphonate

\begin{tabular}{|c|c|c|c|}
\hline 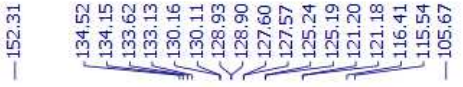 & 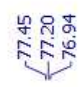 & 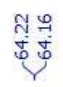 & 虽 \\
\hline
\end{tabular}
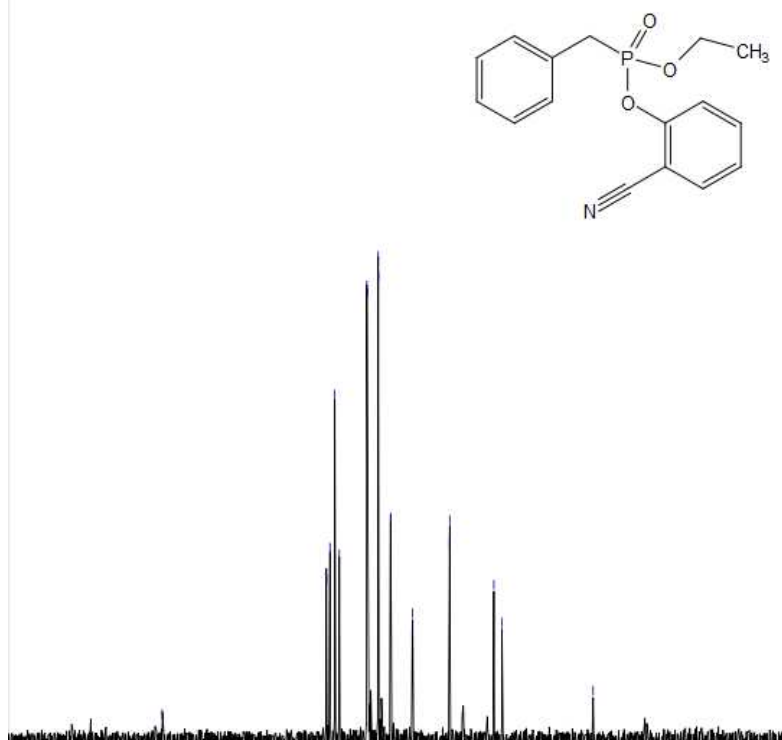

minguming

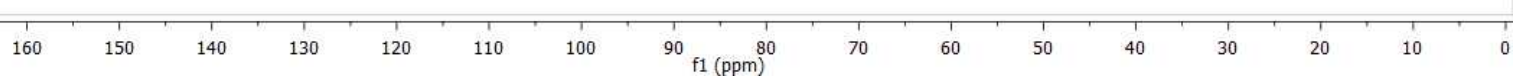


2-cyano-4-methoxyphenyl ethyl benzylphosphonate (1a'):
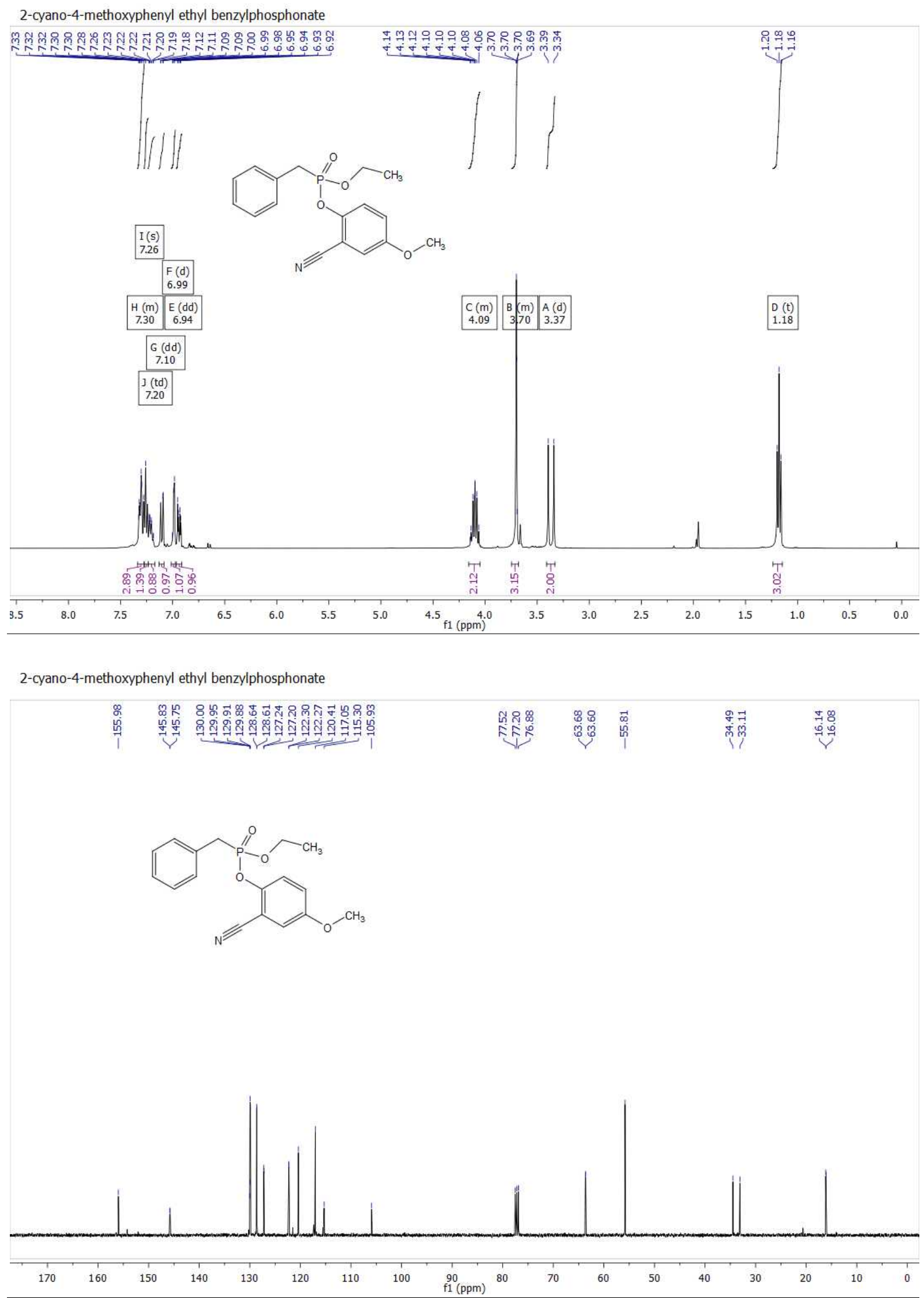

49 
2-cyanophenyl ethyl 4-methylbenzylphosphonate (1b):
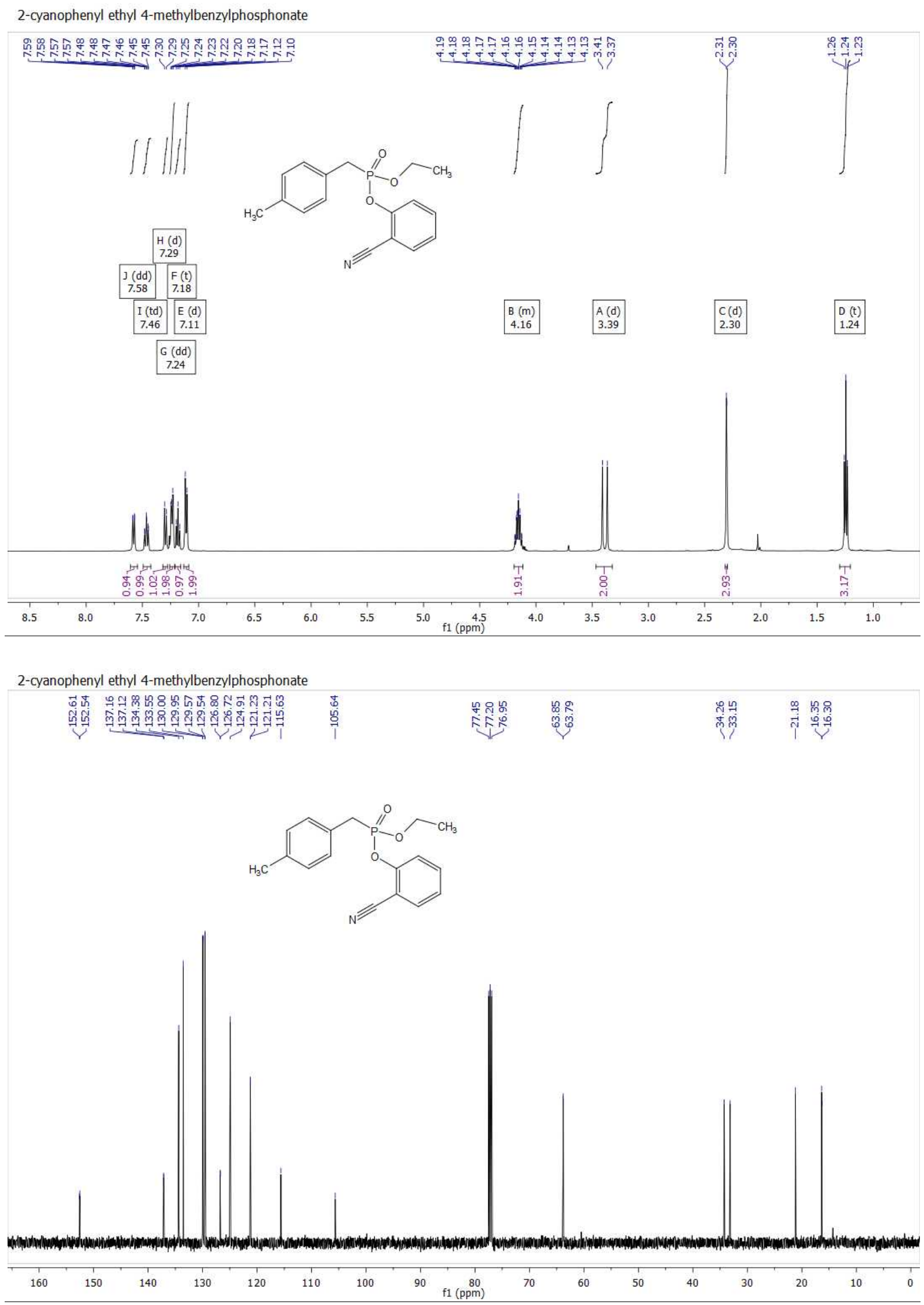


\section{2-cyanophenyl ethyl 4-methoxybenzylphosphonate (1c):}

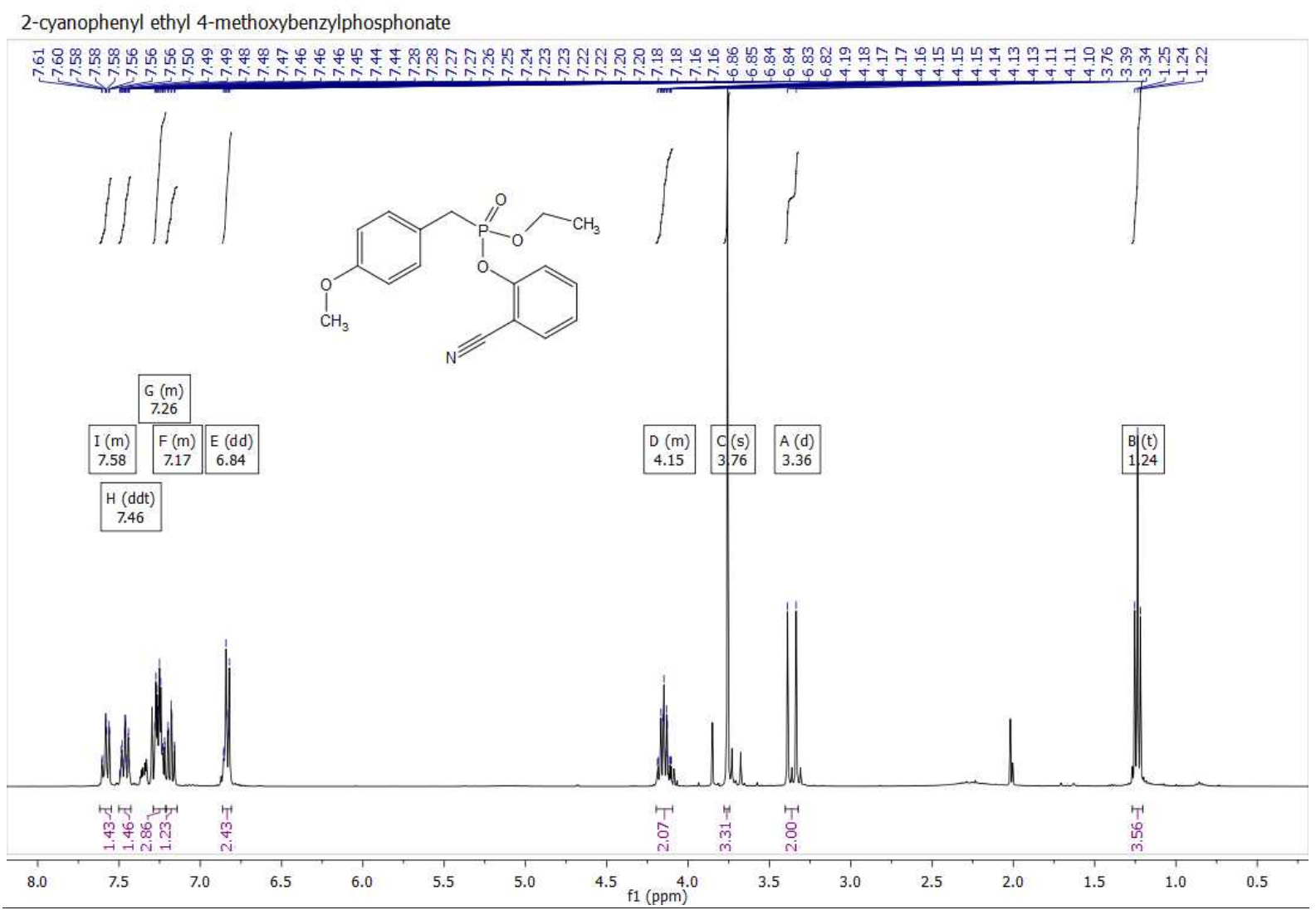

2-cyanophenyl ethyl 4-methoxybenzylphosphonate

\begin{tabular}{|c|c|c|c|c|c|c|}
\hline 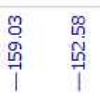 & 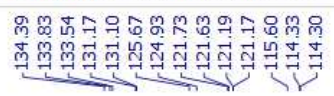 & 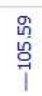 & 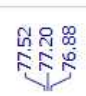 & 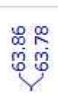 & 崔 & 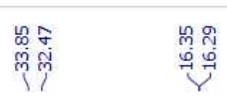 \\
\hline
\end{tabular}<smiles>CCOP(=O)(Cc1ccc(OC)cc1)Oc1ccccc1C#N</smiles>

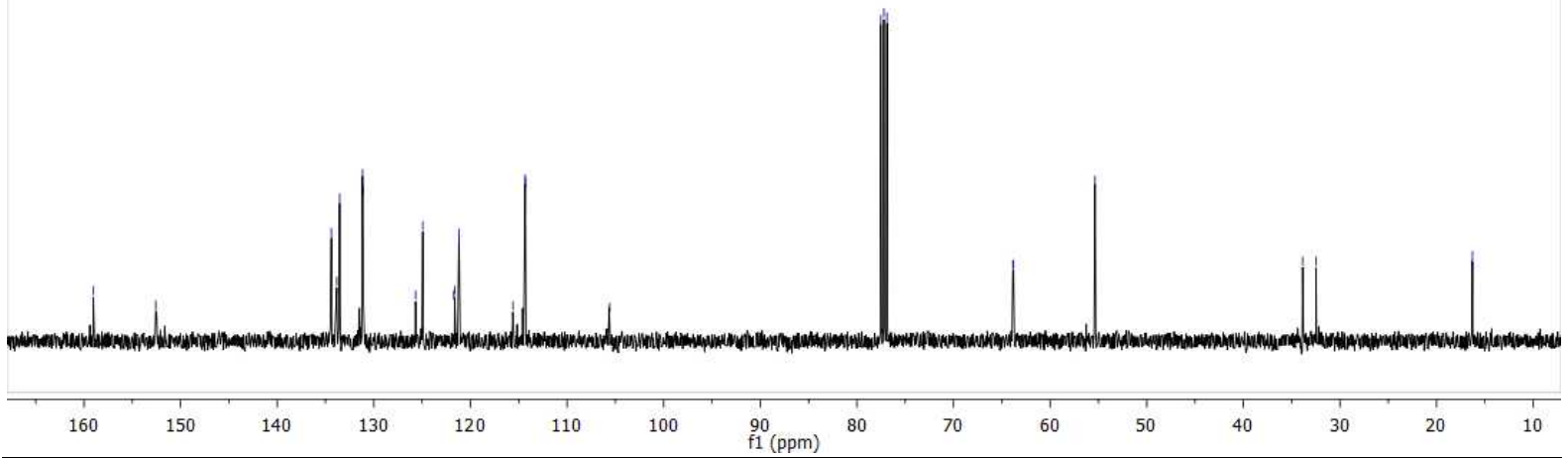


2-cyanophenyl ethyl 4-bromobenzylphosphonate (1d):

2-cyanophenyl ethyl 4-bromobenzylphosphonate

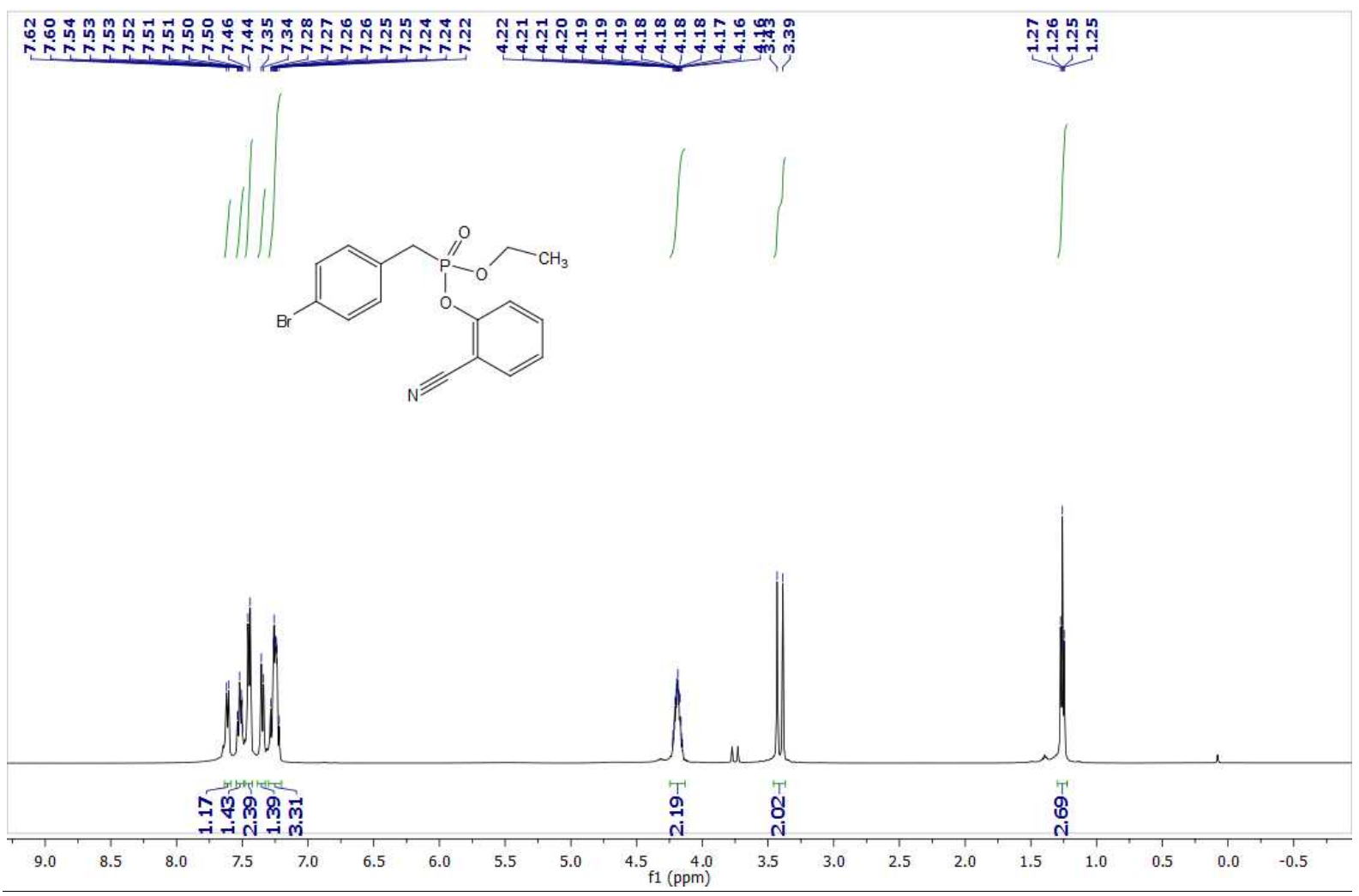

2-cyanophenyl ethyl 4-bromobenzylphosphonate

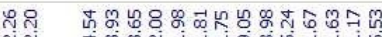

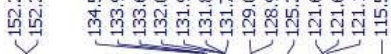

占

mृ

ma<smiles>CCOP(=O)(Cc1ccc(Br)cc1)Oc1ccccc1C#N</smiles>

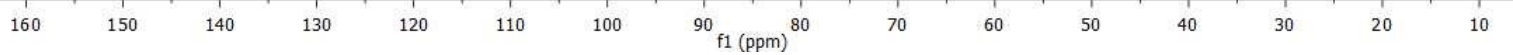


2-cyanophenyl ethyl 2-bromobenzylphosphonate (1e):

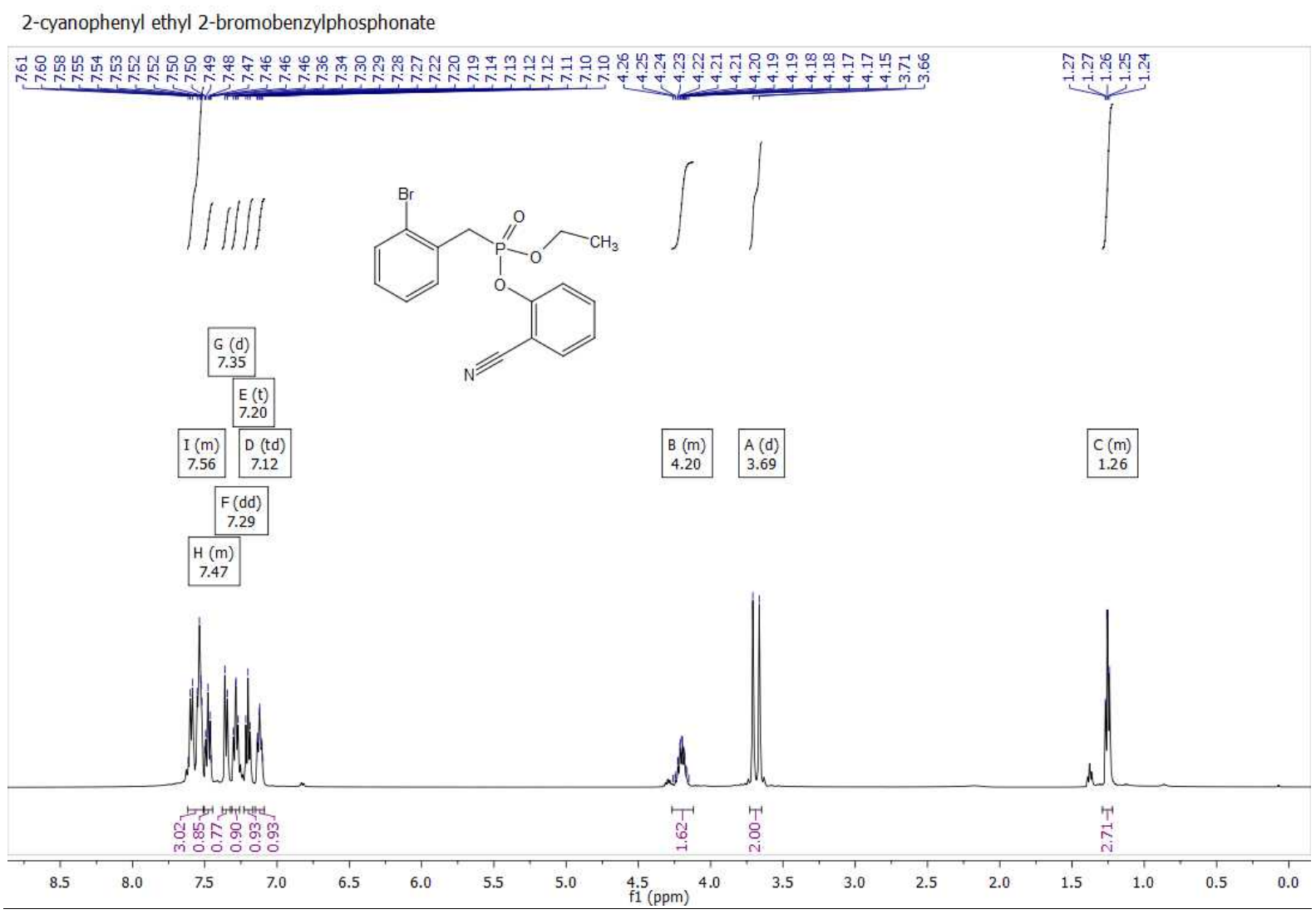

2-cyanophenyl ethyl 2-bromobenzylphosphonate

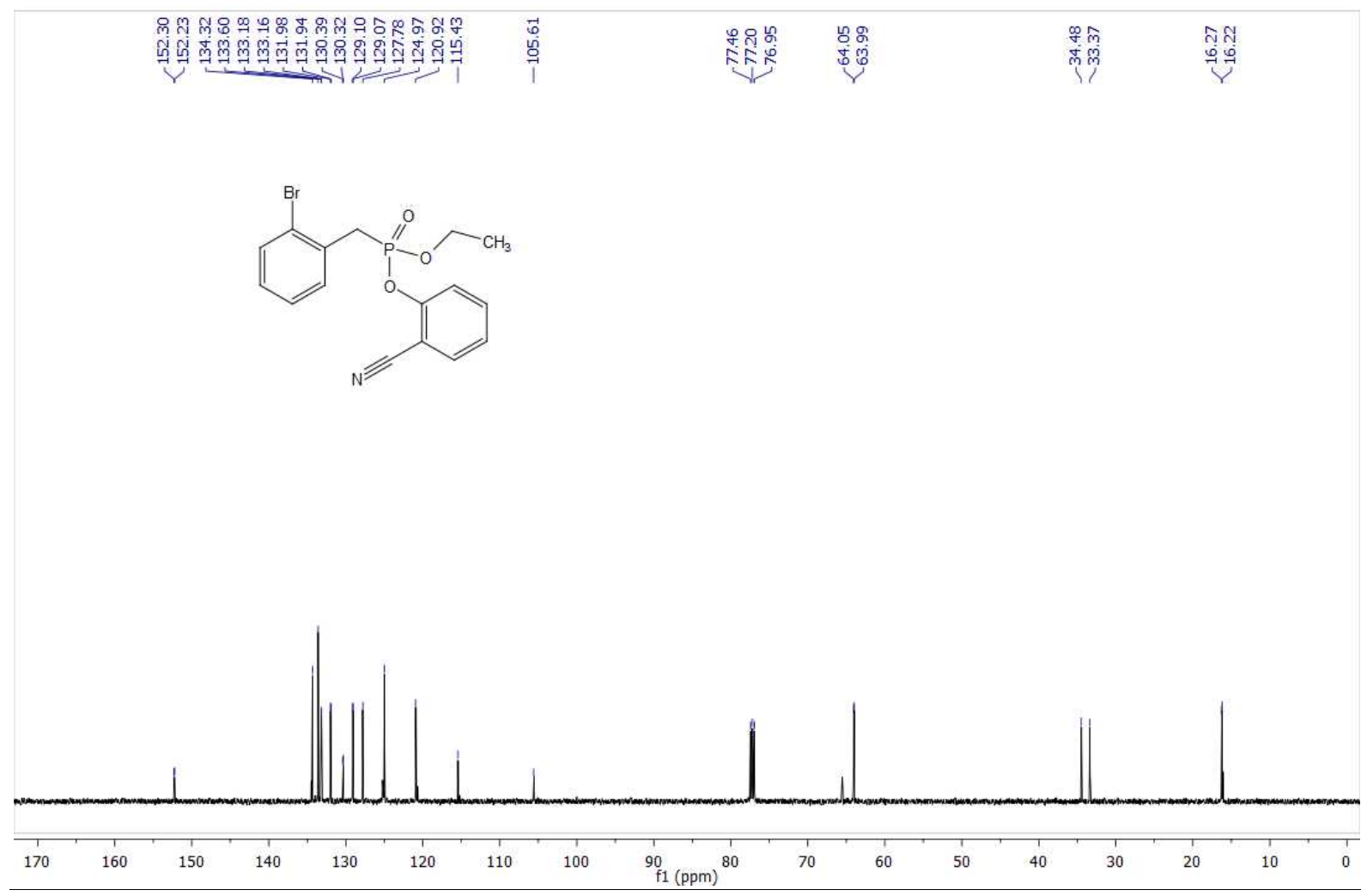

53 
2-cyanophenyl ethyl 2,5-difluorobenzylphosphonate (1f):

2-cyanophenyl ethyl 2,5-difluorobenzylphosphonate

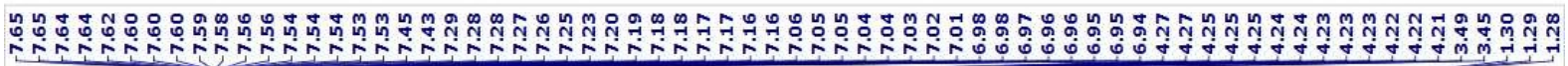

\|\|\|\|
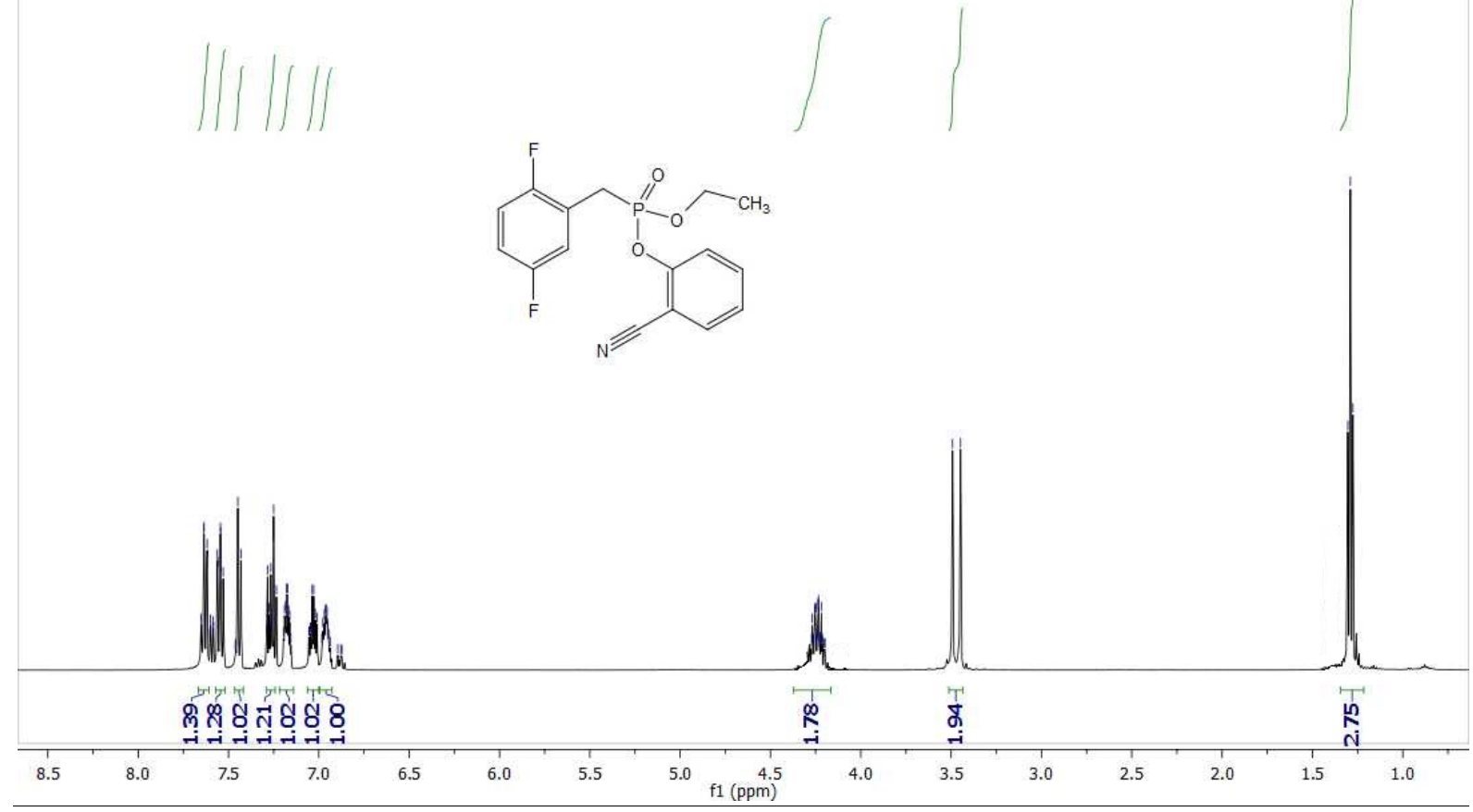

2-cyanophenyl ethyl 2,5-difluorobenzylphosphonate

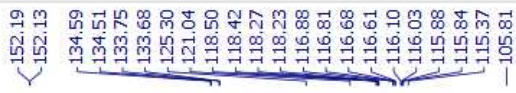

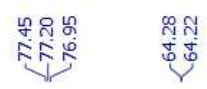

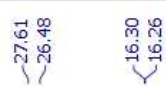
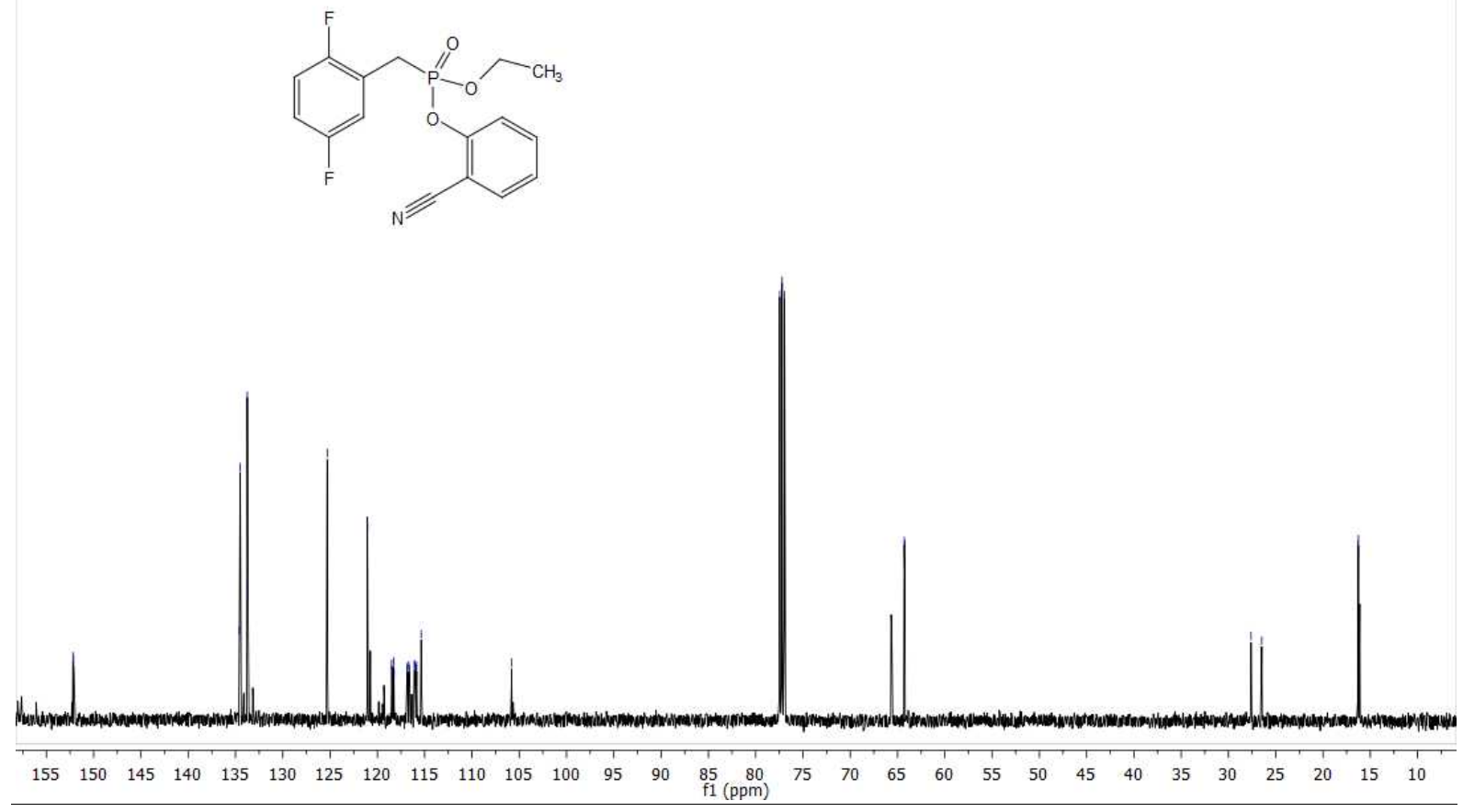

54 
2-cyanophenyl ethyl 3-bromobenzylphosphonate (1g):

2-cyanophenyl ethyl 3-bromobenzylphosphonate

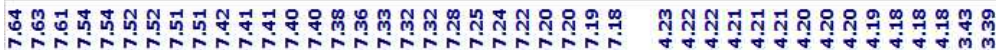

ริจำำำำ
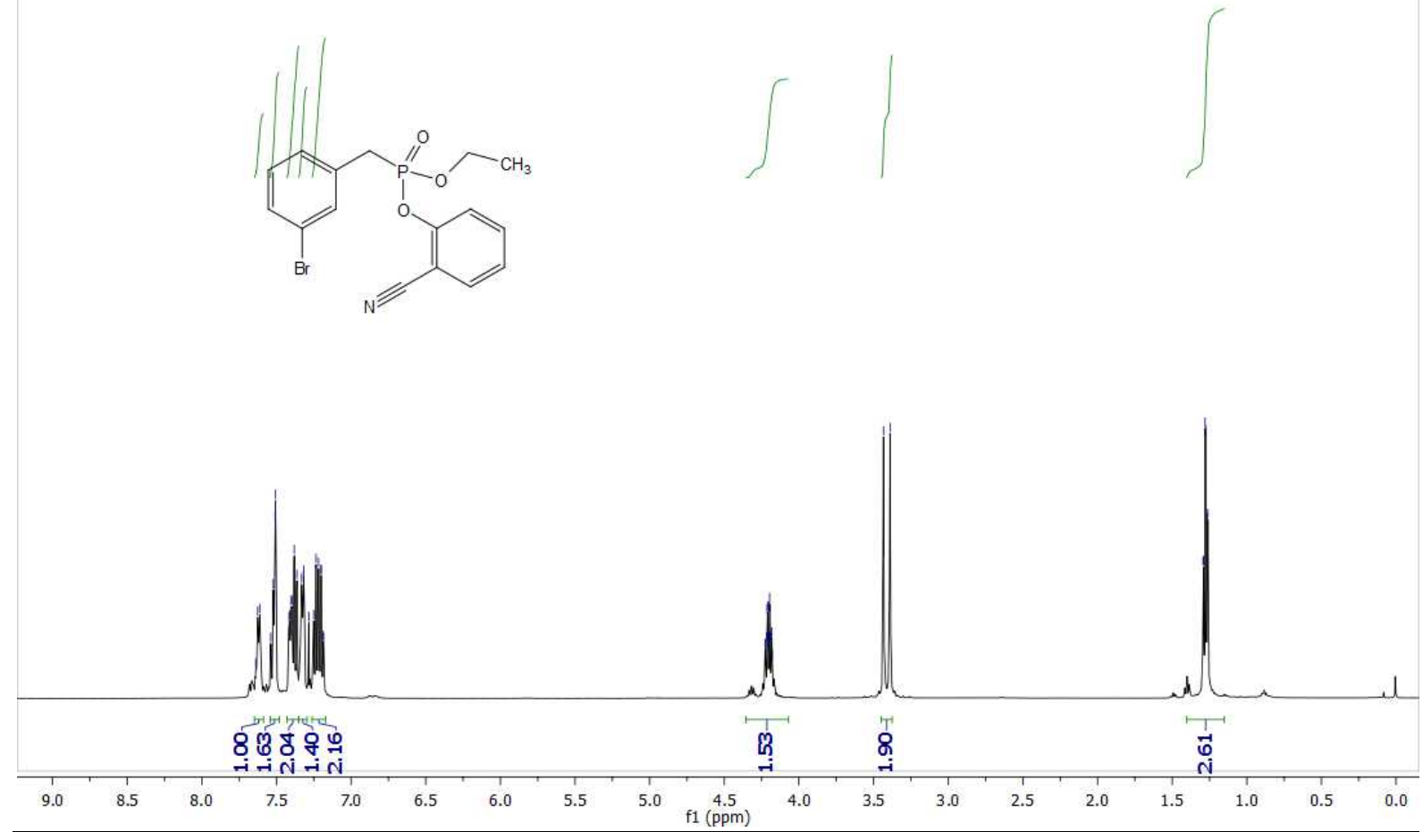

2-cyanophenyl ethyl 3-bromobenzylphosphonate

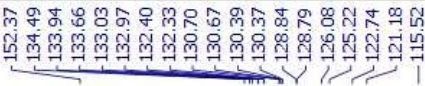

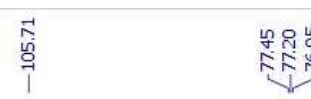

等金

焦

帒
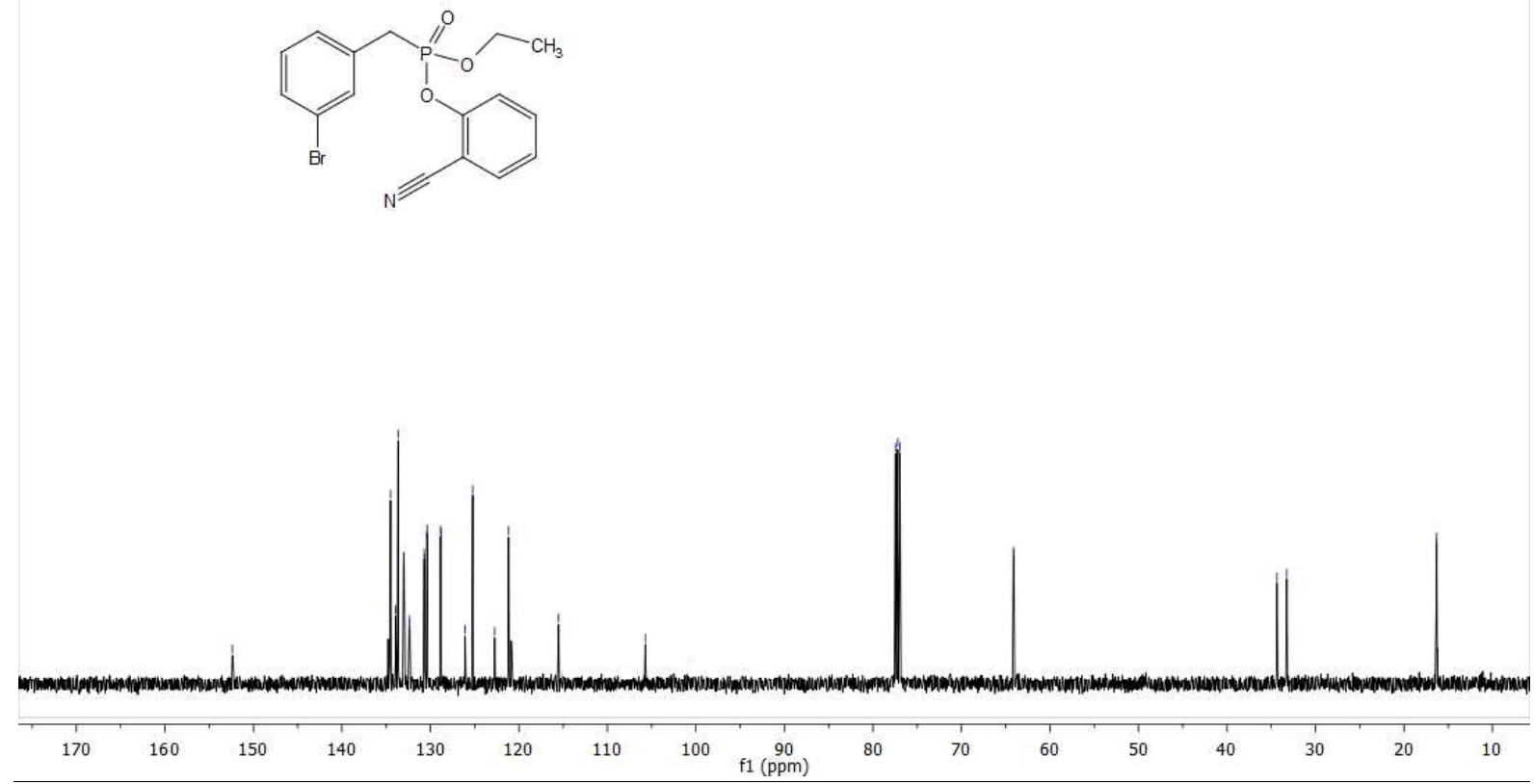

55 
2-cyanophenyl ethyl 3-bromo-4-fluorobenzylphosphonate (1h):

2-cyanophenyl ethyl 3-bromo-4-fluorobenzylphosphonate

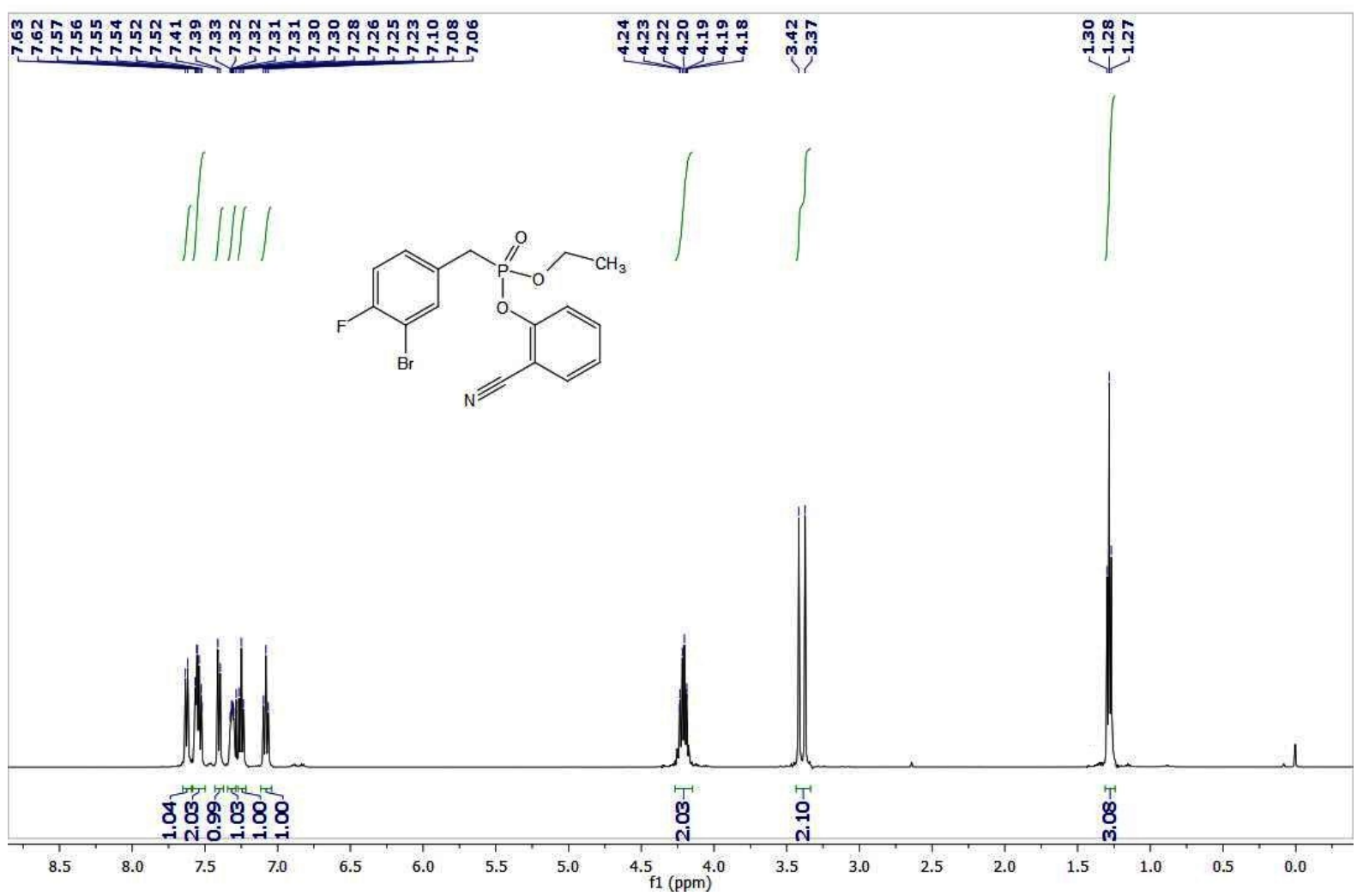

2-cyanophenyl ethyl 3-bromo-4-fluorobenzylphosphonate

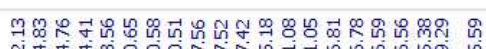

ํํำ
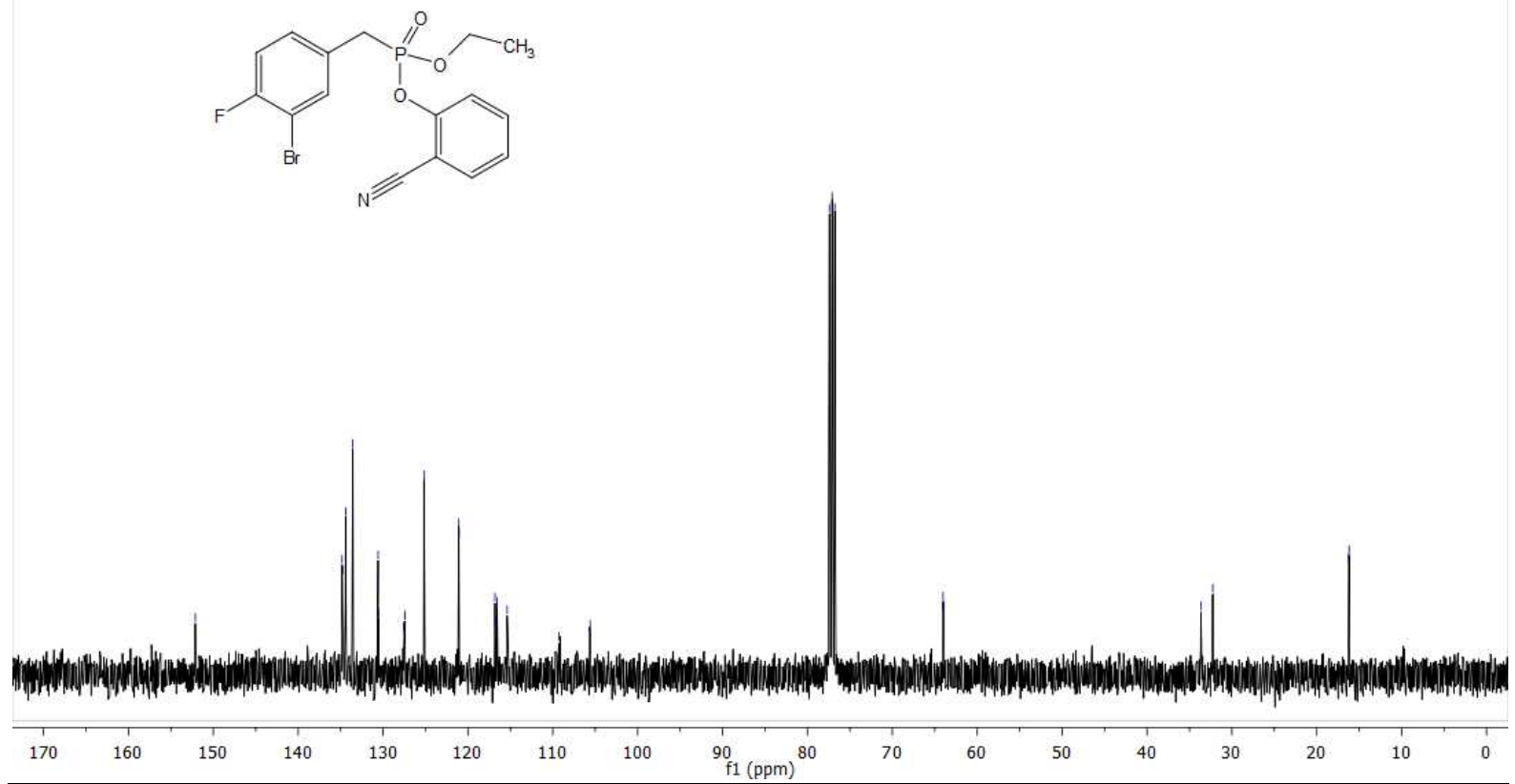


\section{2-cyanophenyl ethyl 2-methylbenzylphosphonate (1i):}

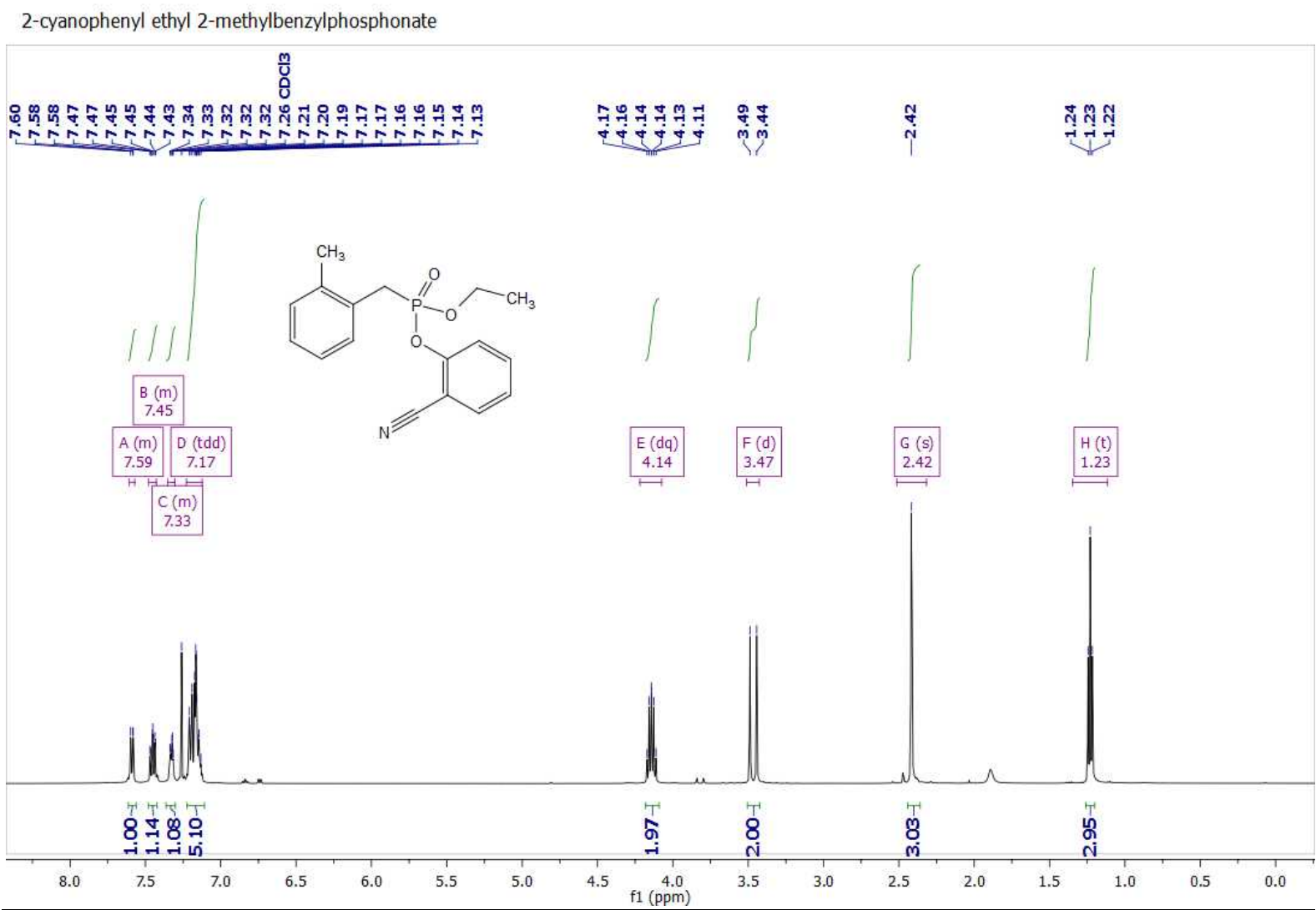

2-cyanophenyl ethyl 2-methylbenzylphosphonate

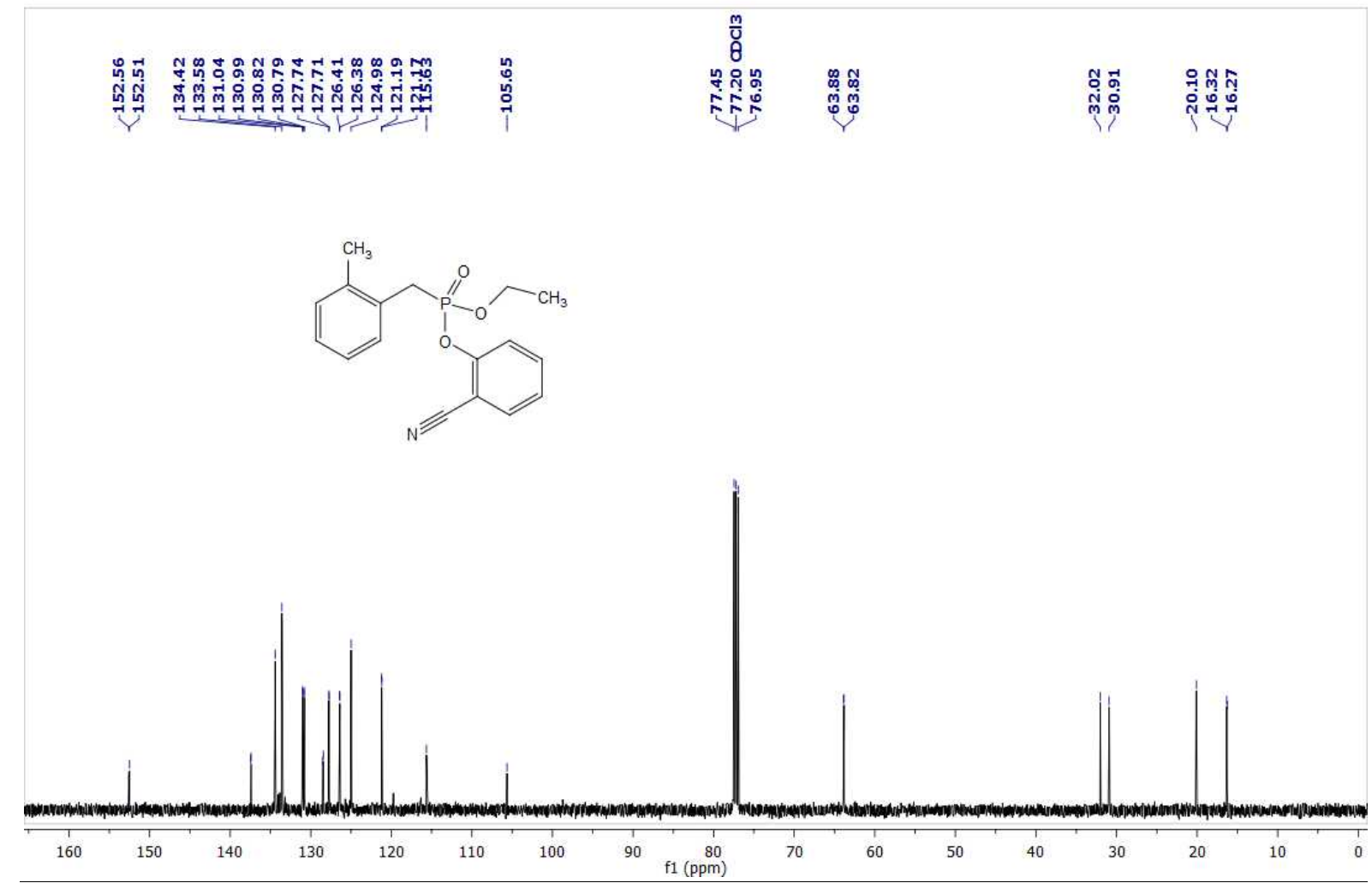


2-cyanophenyl ethyl 3-methylbenzylphosphonate (1j):

2-cyanophenyl ethyl 3-methylbenzylphosphonate

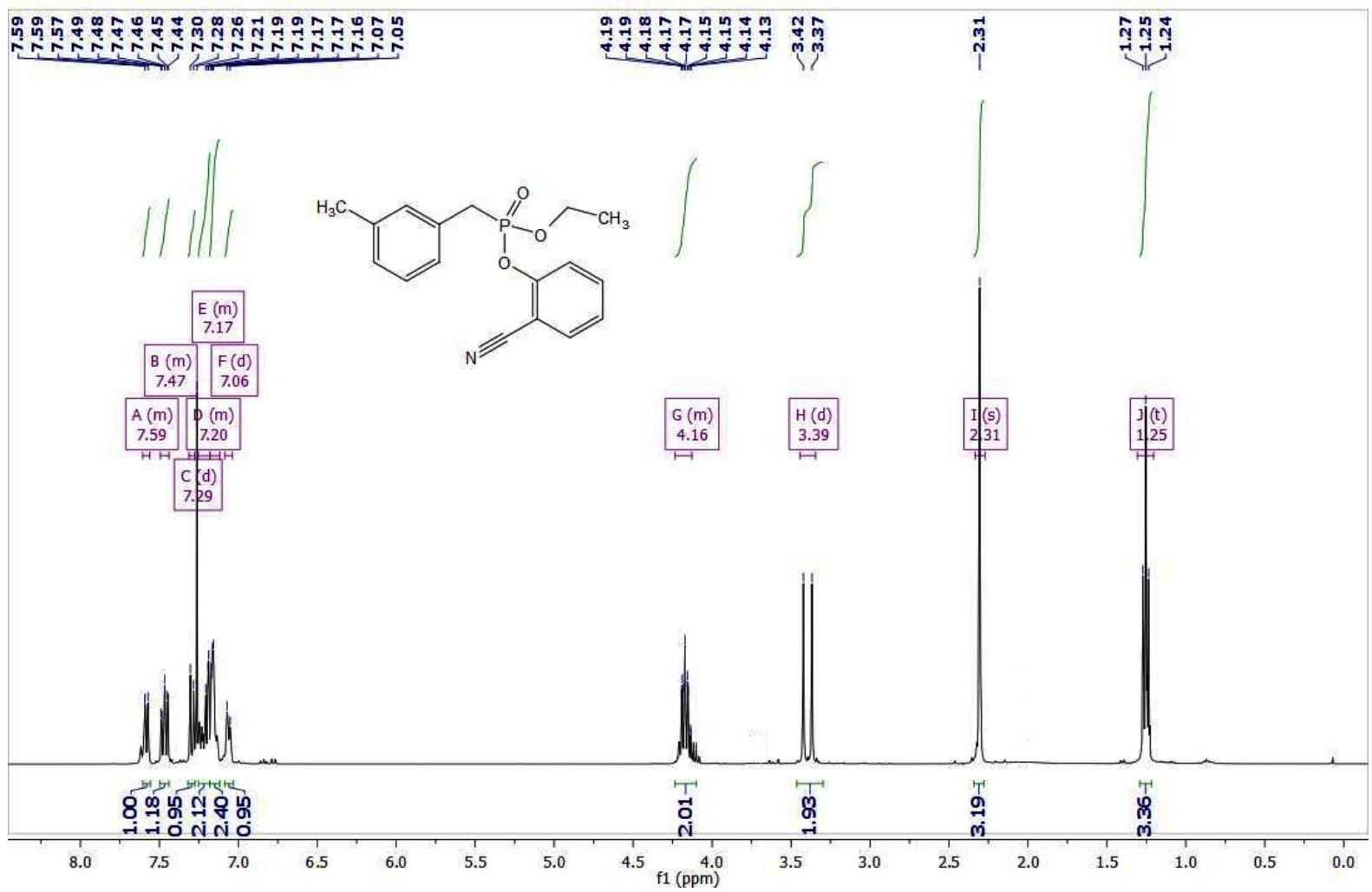

2-cyanophenyl ethyl 3-methylbenzylphosphonate

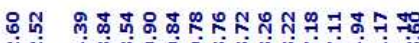

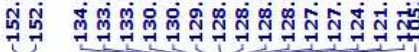

$\frac{\mathrm{m}}{8}$

忥

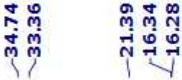<smiles>CCOP(=O)(Cc1cccc(C)c1)Oc1ccccc1C#N</smiles>

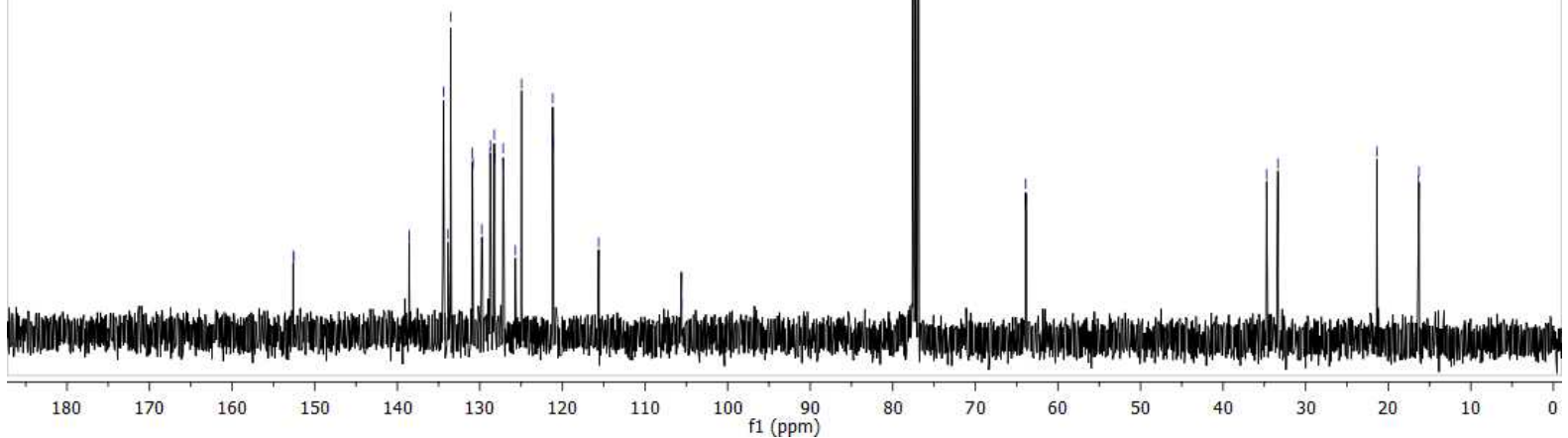


2-cyanophenyl ethyl 4-tert-butylbenzylphosphonate (1k):

2-cyanophenyl ethyl 4-tert-butylbenzylphosphonate

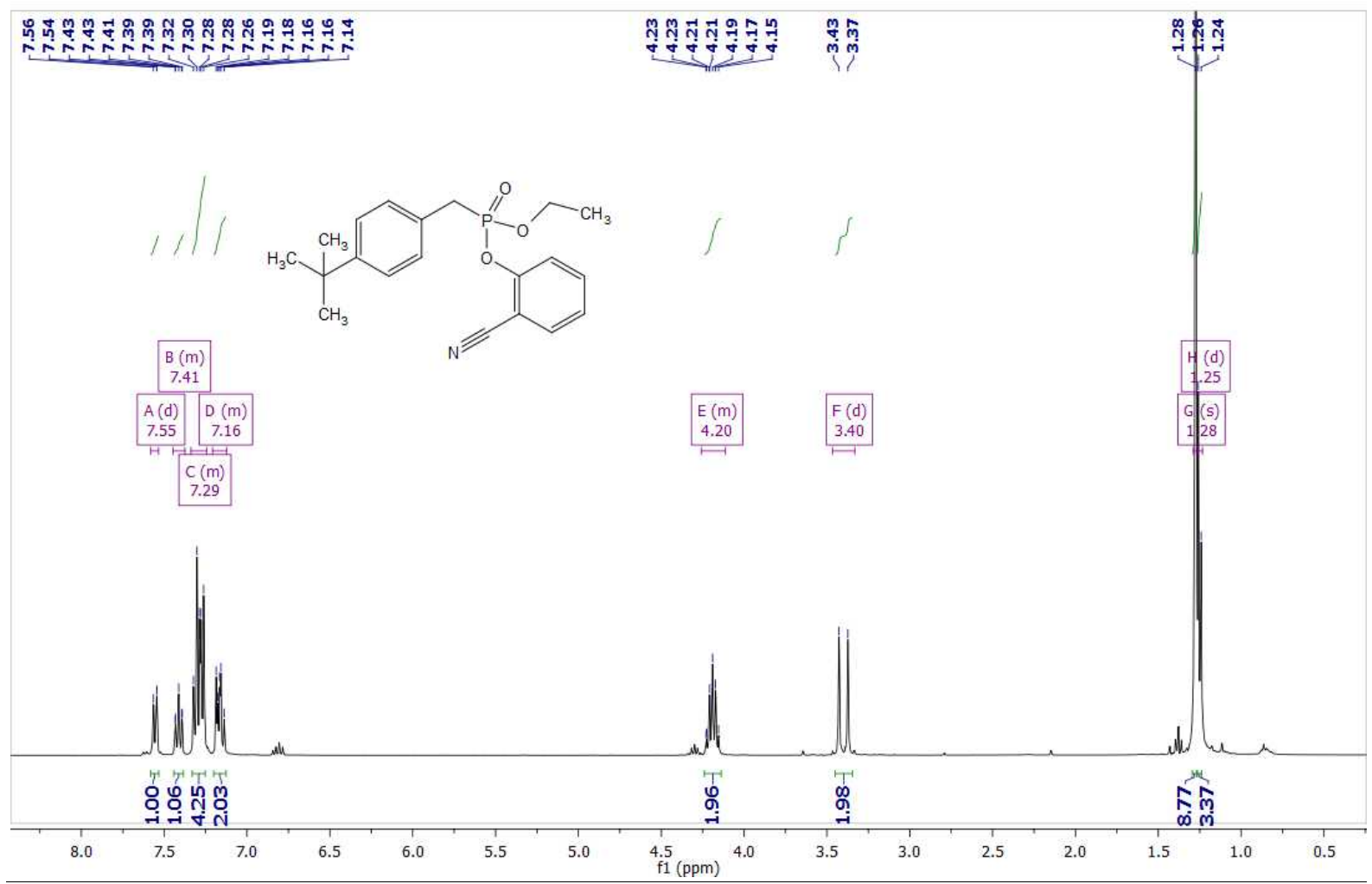

2-cyanophenyl ethyl 4-tert-butylbenzylphosphonate

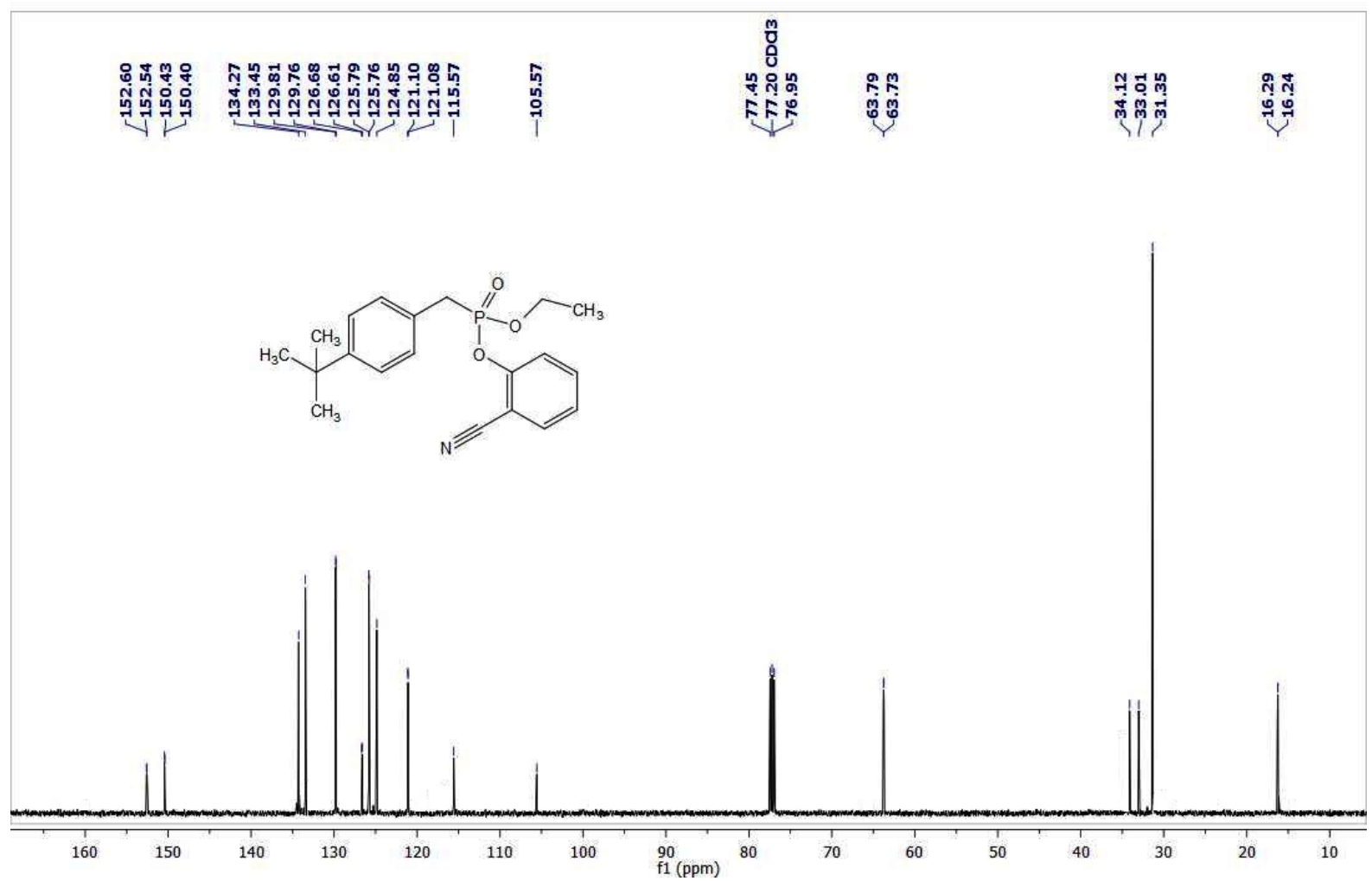




\section{Mono-Olefinated Products:}

(E)-ethyl 3-(3-(((2-cyanophenoxy)(ethoxy)phosphoryl)methyl)phenyl)acrylate (2a):

(E)-ethyl 3-(3-(((2-cyanophenoxy)(ethoxy)phosphoryl)methyl)phenyl)acrylate

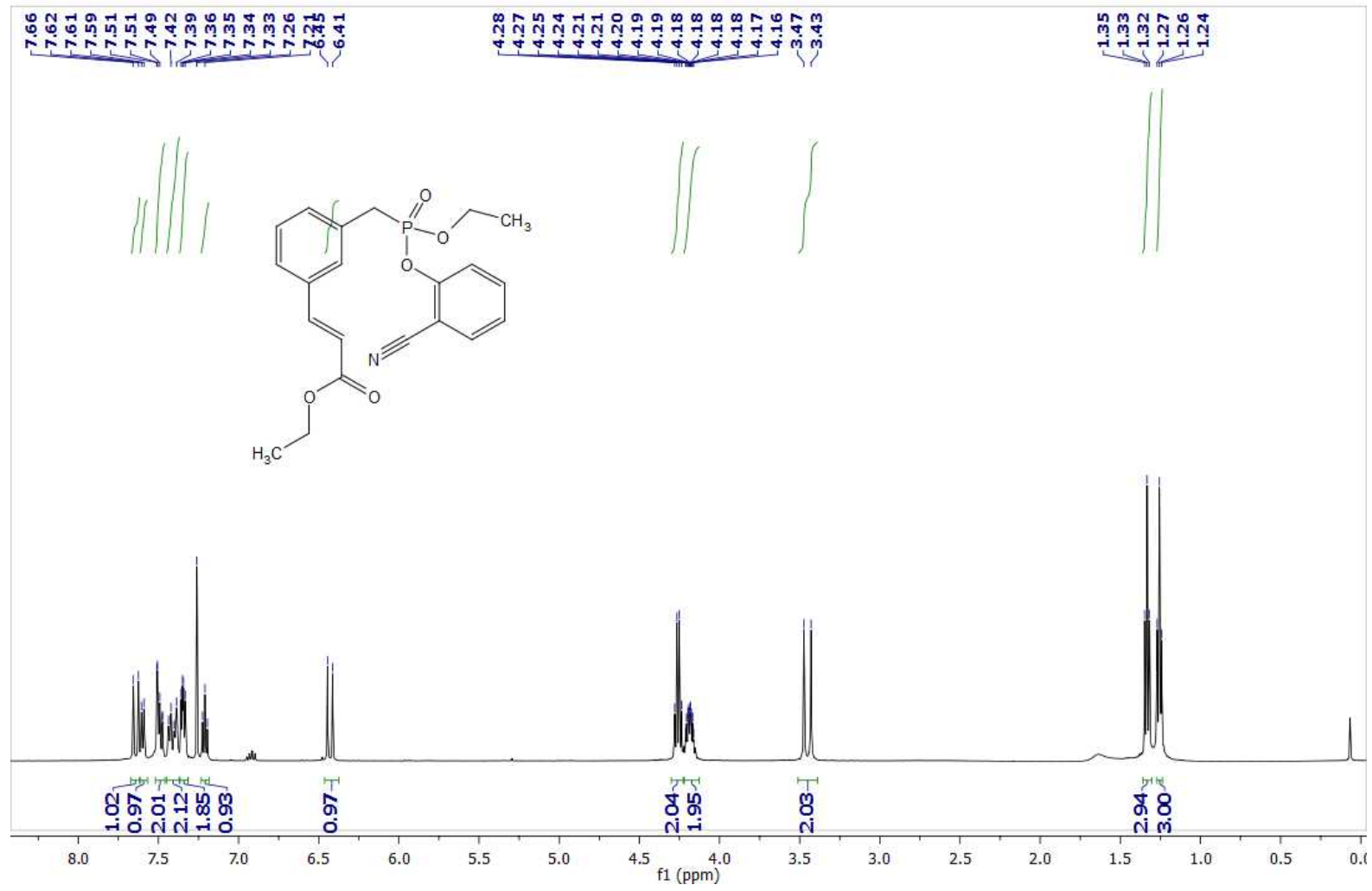

(E)-ethyl 3-(3-(((2-cyanophenoxy)(ethoxy)phosphoryl)methyl)phenyl)acrylate

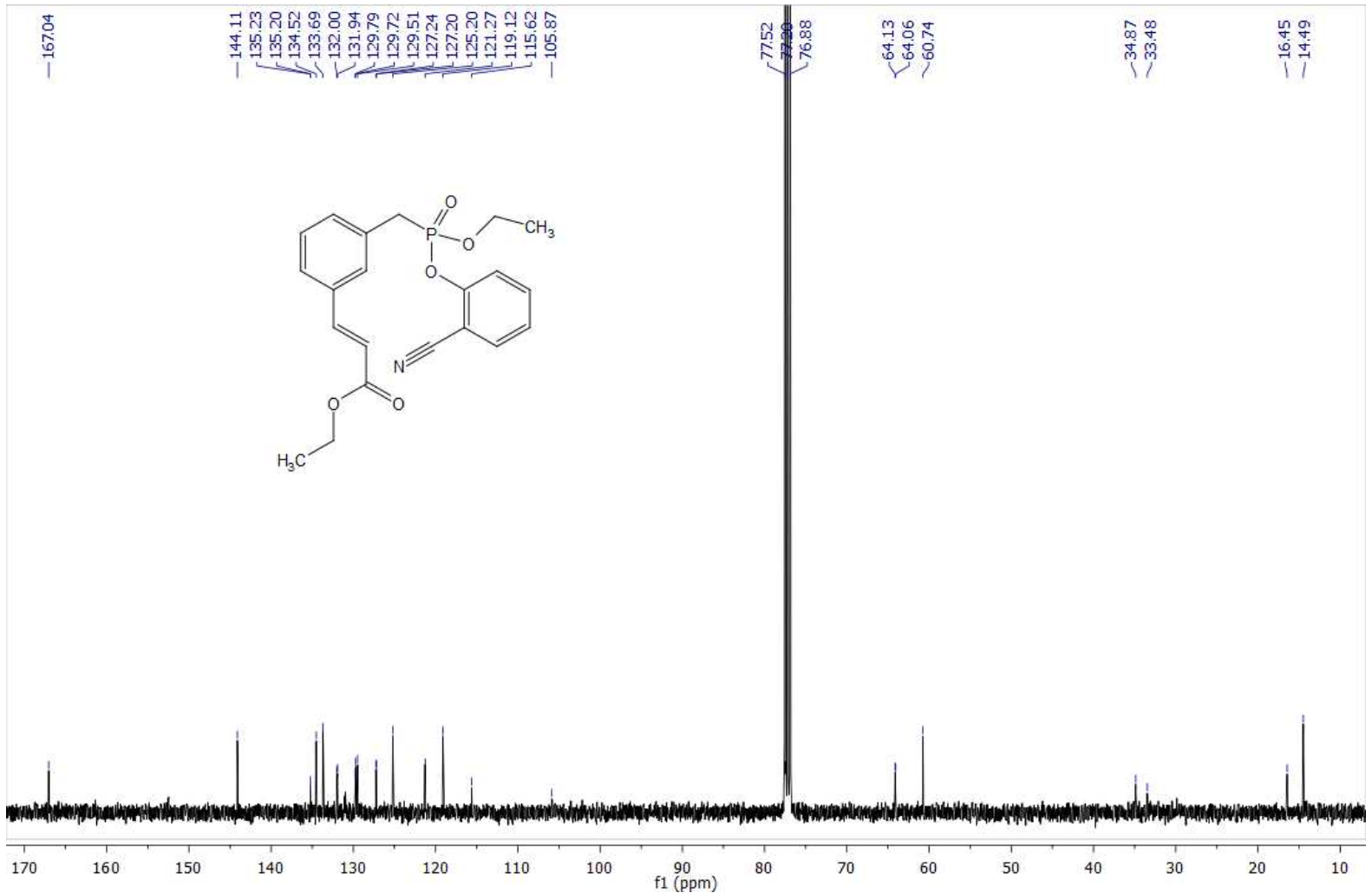


(E)-methyl 3-(3-(((2-cyanophenoxy)(ethoxy)phosphoryl)methyl)phenyl)acrylate(2b):

(E)-methyl 3-(3-(((2-cyanophenoxy)(ethoxy)phosphoryl)methyl)phenyl)acrylate

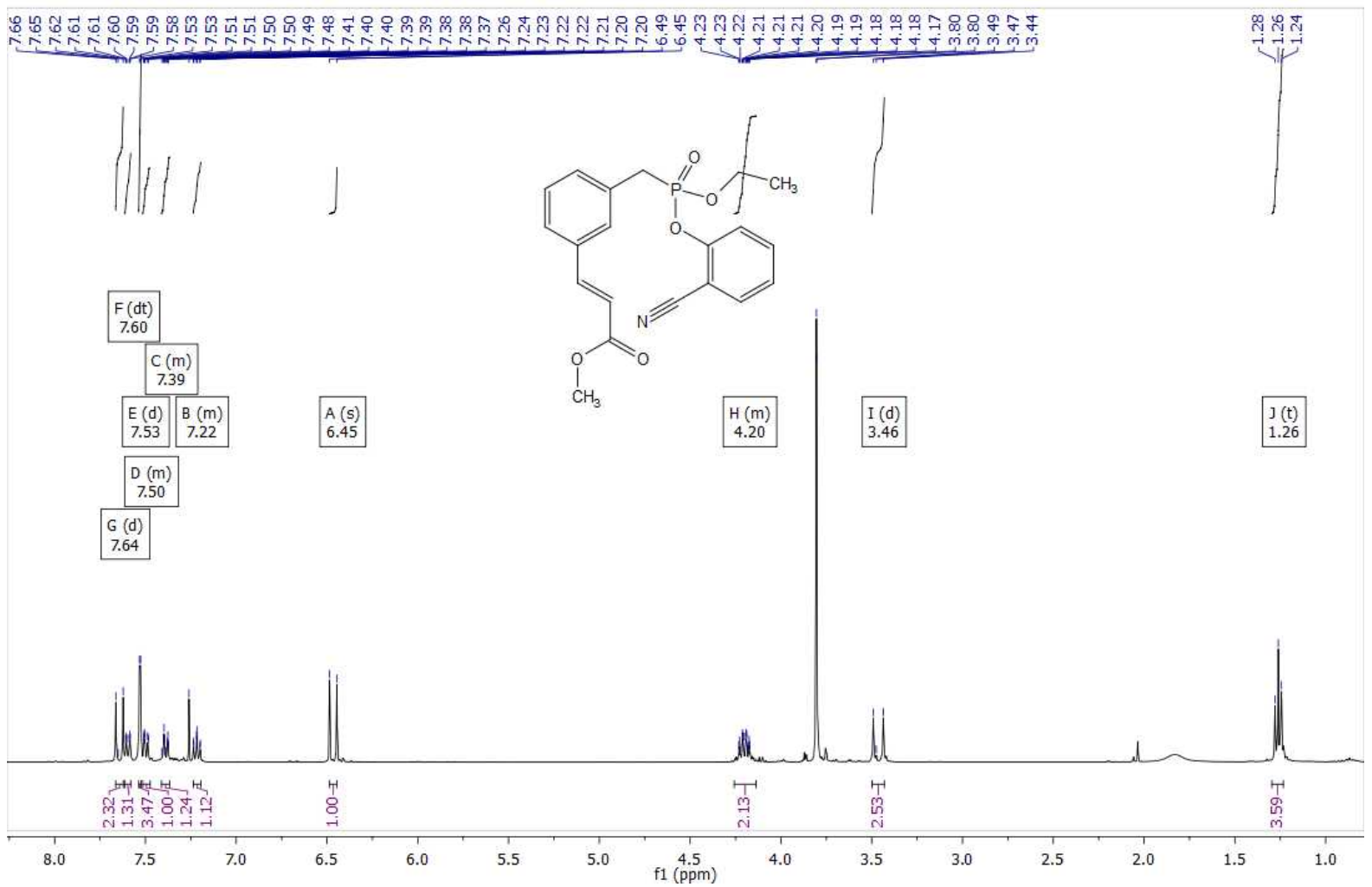

(E)-methyl 3-(3-(((2-cyanophenoxy)(ethoxy)phosphoryl)methyl)phenyl)acrylate

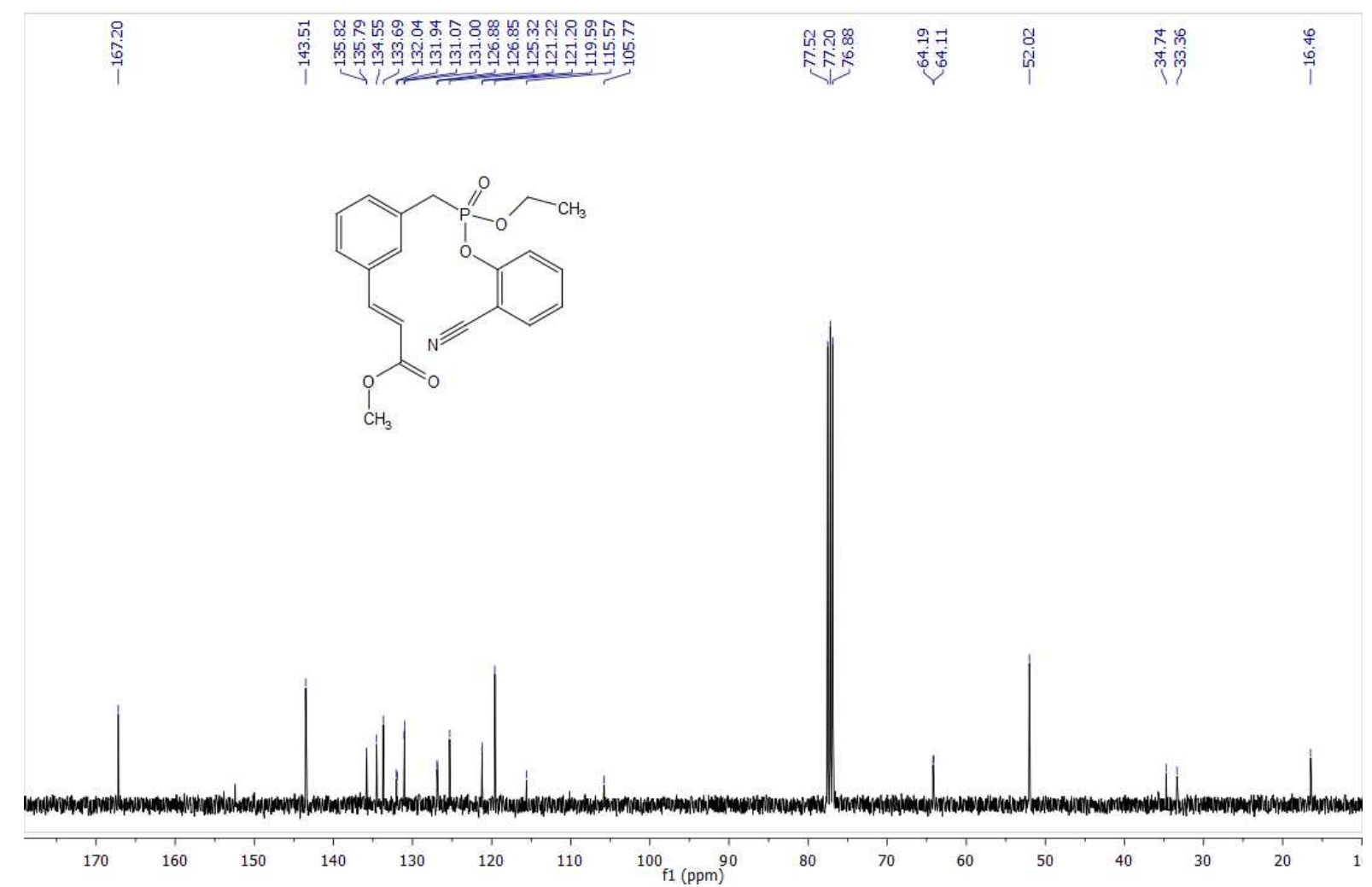


(E)-benzyl 3-(3-(((2-cyanophenoxy)(ethoxy)phosphoryl)methyl)phenyl)acrylate (2c):

(E)-benzyl 3-(3-(((2-cyanophenoxy)(ethoxy)phosphoryl)methyl)phenyl)acrylate

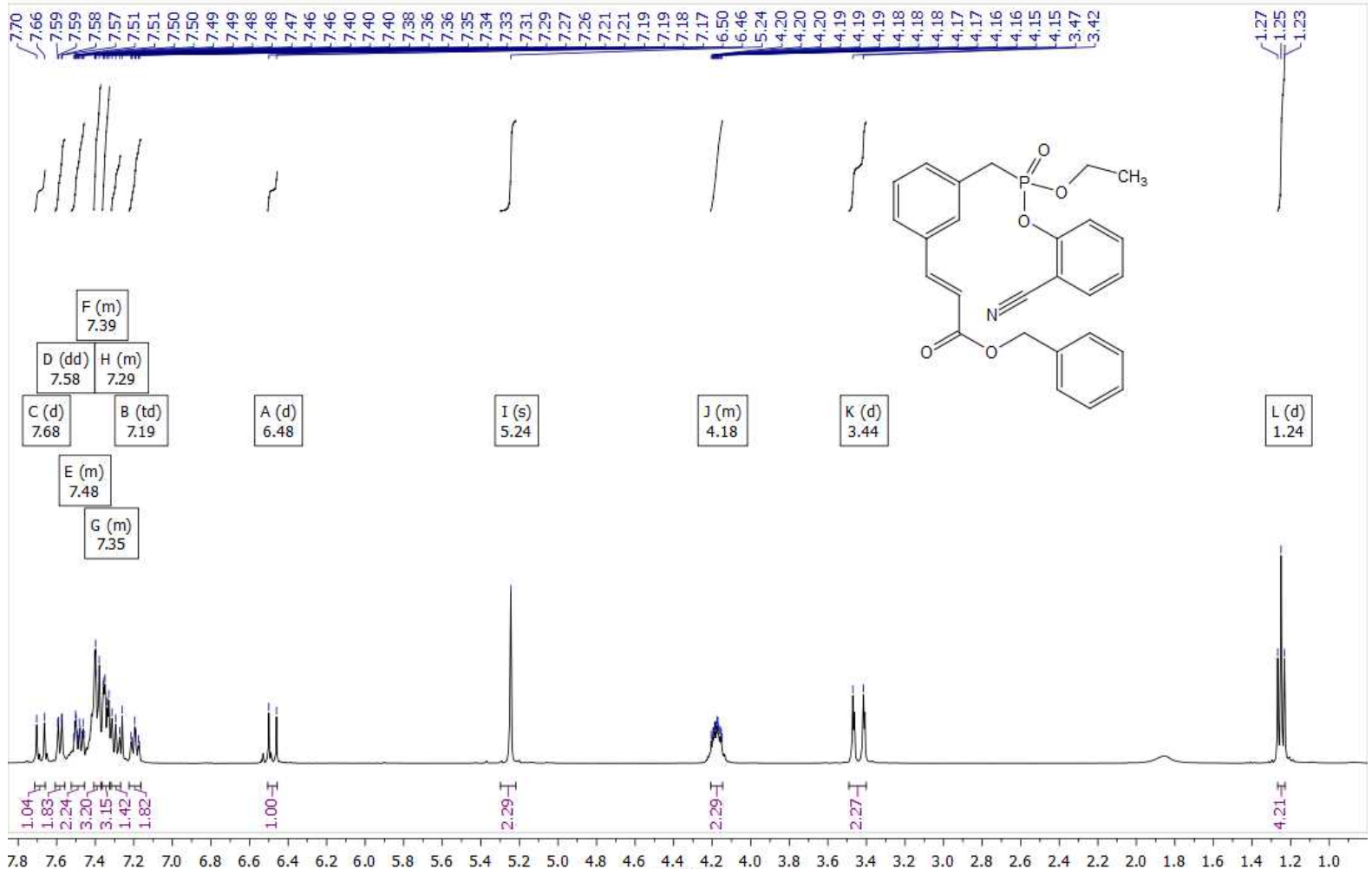

(E)-benzyl 3-(3-(((2-cyanophenoxy)(ethoxy)phosphoryl)methyl)phenyl)acrylate

\begin{tabular}{|c|c|c|}
\hline 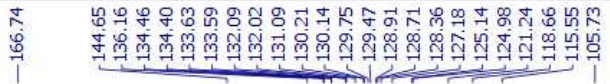 & 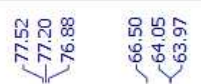 & 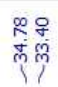 \\
\hline
\end{tabular}<smiles>CCOP(=O)(Cc1cccc(/C=C/C(=O)OCc2ccccc2)c1)Oc1ccccc1C#N</smiles>

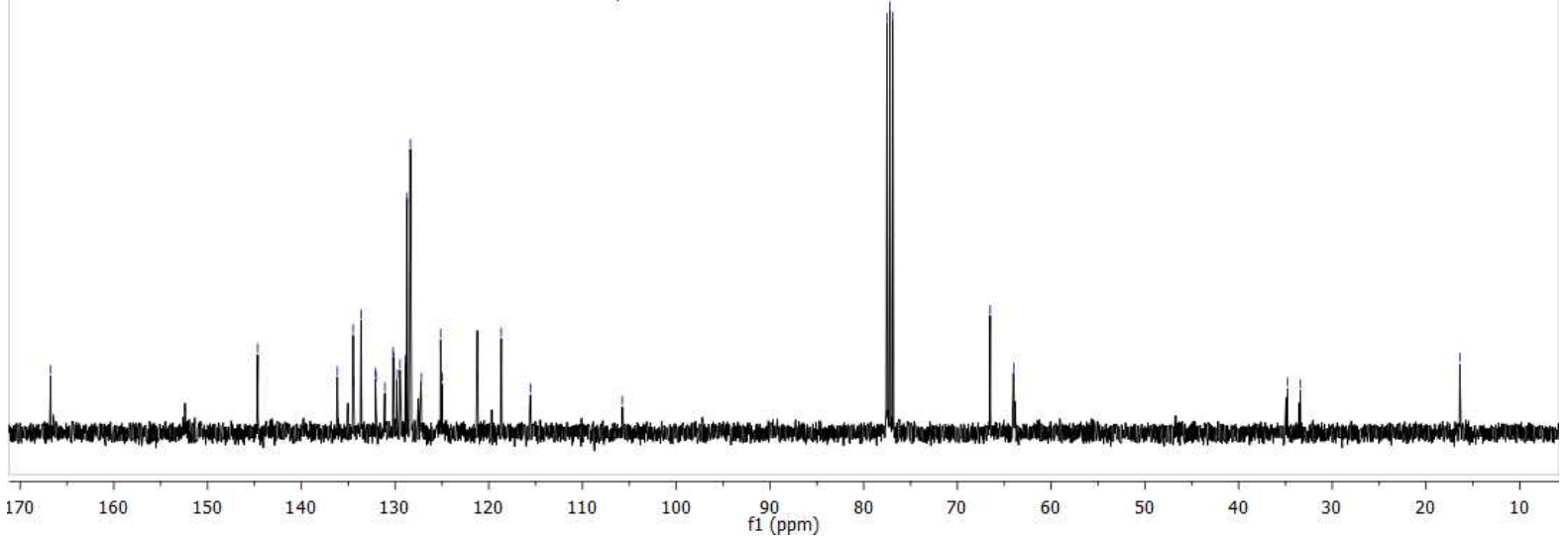


(E)-2-cyanophenyl ethyl 3-(3-oxobut-1-en-1-yl)benzylphosphonate (2d):

(E)-2-cyanophenyl ethyl 3-(3-oxobut-1-en-1-yl)benzylphosphonate

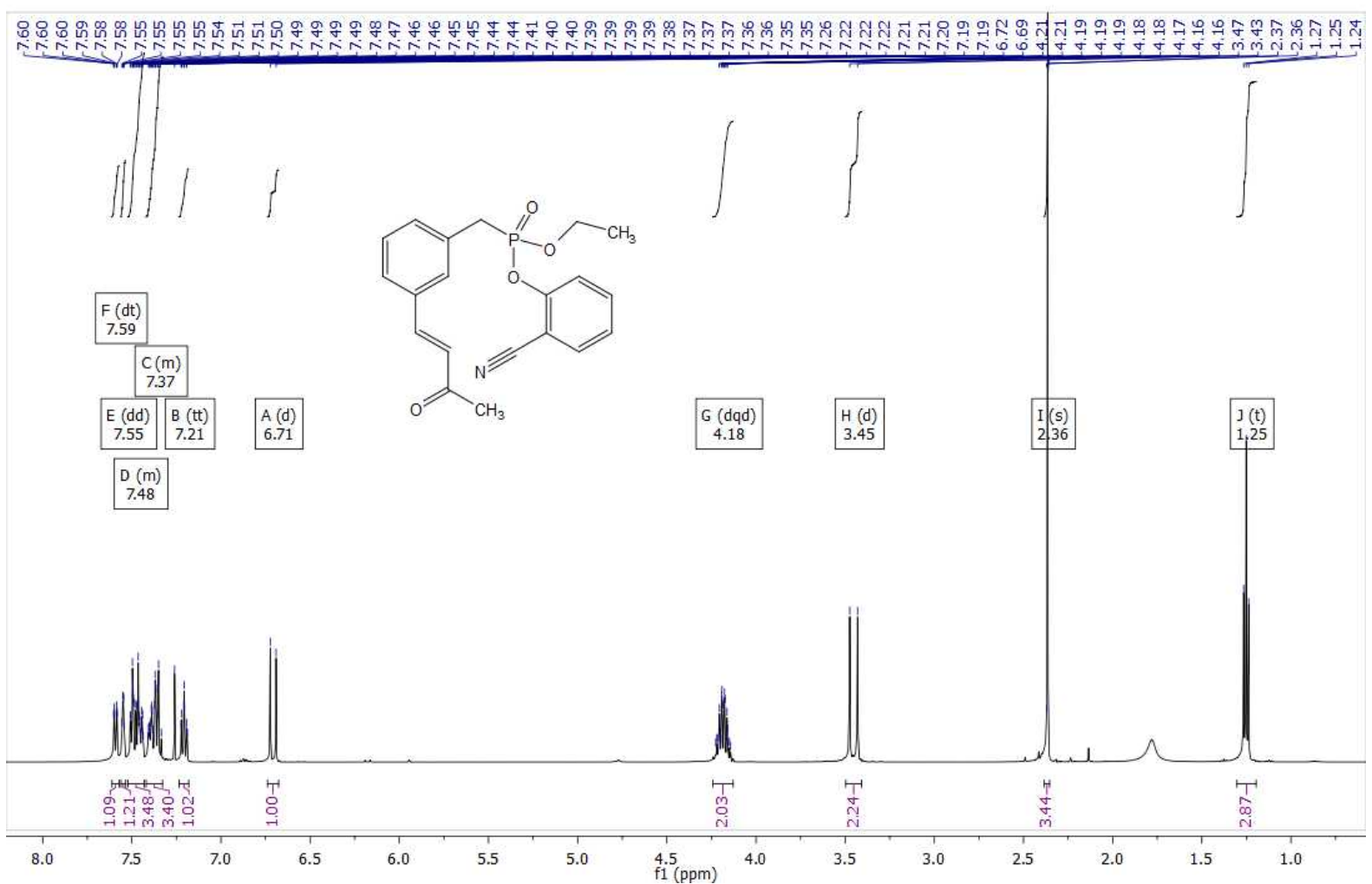

(E)-2-cyanophenyl ethyl 3-(3-oxobut-1-en-1-yl)benzylphosphonate

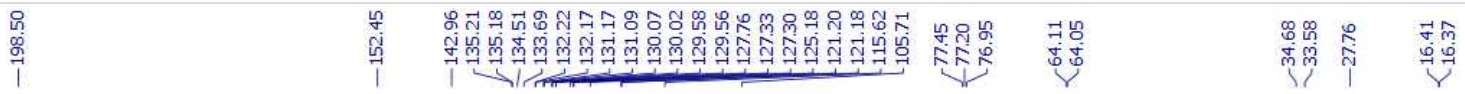<smiles>CCOP(=O)(Cc1cccc(/C=C/C(C)=O)c1)Oc1ccccc1C#N</smiles>

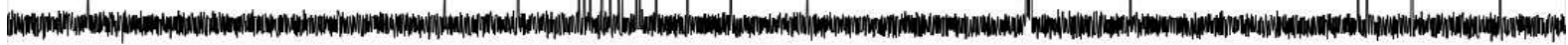

200 110 f1 (ppm) 100 
(E)-2-cyanophenyl ethyl 3-(2-(diethoxyphosphoryl)vinyl)benzylphosphonate (2e):

(E)-2-cyanophenyl ethyl 3-(2-(diethoxyphosphoryl)vinyl)benzylphosphonate

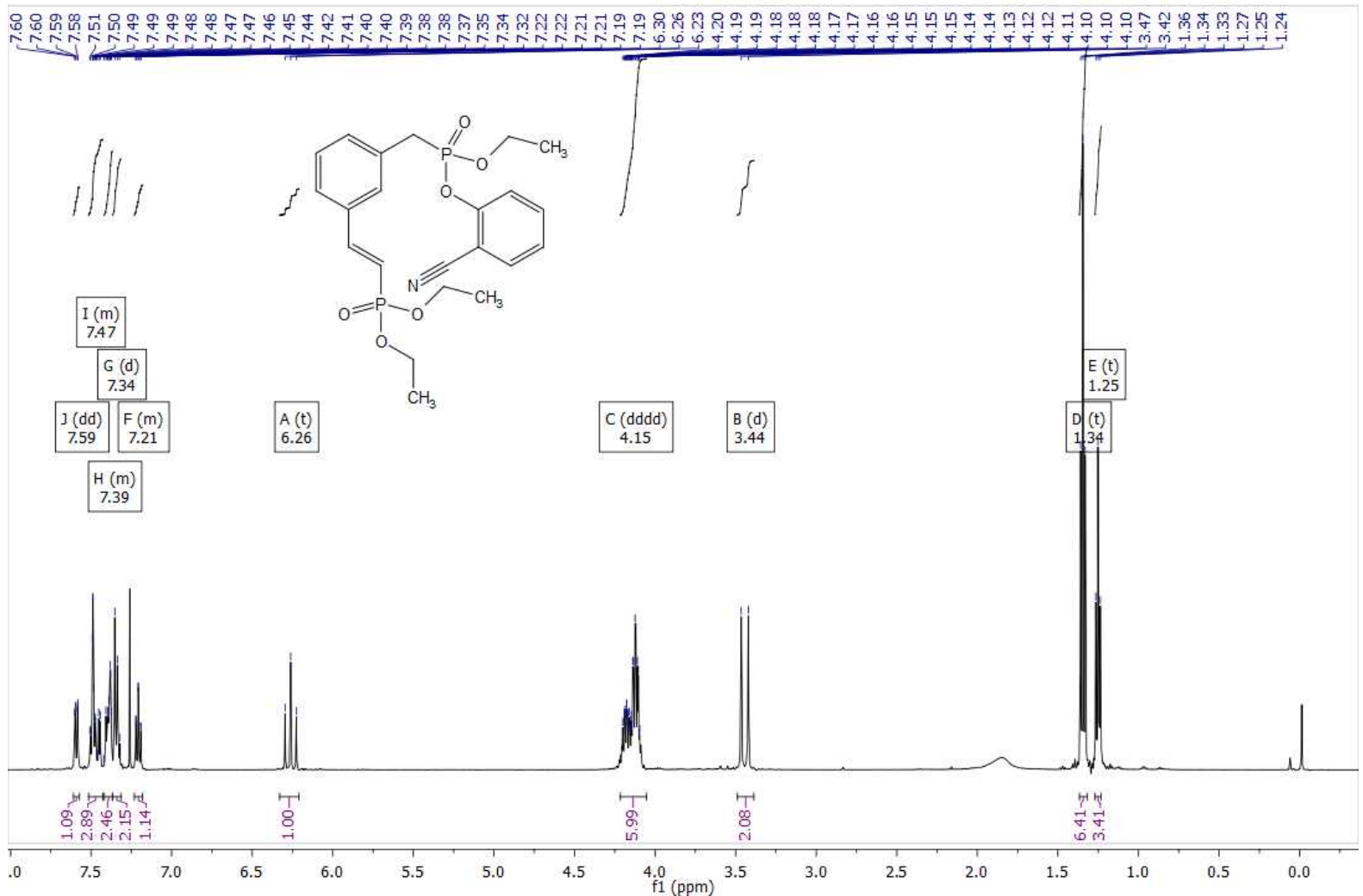

(E)-2-cyanophenyl ethyl 3-(2-(diethoxyphosphoryl)vinyl)benzylphosphonate

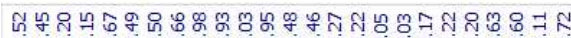

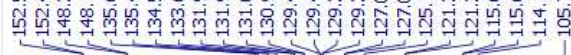<smiles>CCOP(=O)(/C=C/c1cccc(CP(=O)(OCC)OCC)c1)Oc1ccccc1C#N</smiles>

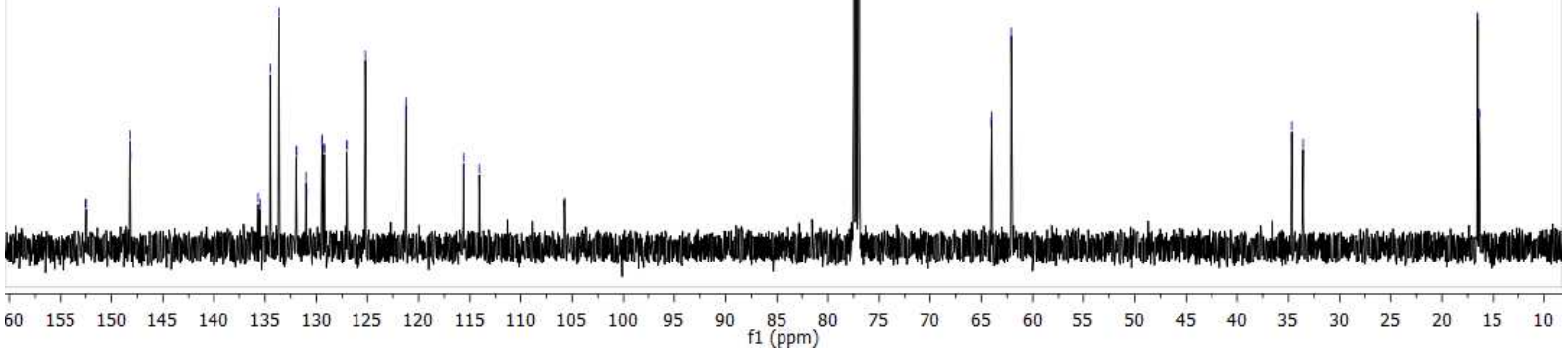


(E)-2-cyanophenyl ethyl 3-(2-(methylsulfonyl)vinyl)benzylphosphonate (2f):

(E)-2-cyanophenyl ethyl 3-(2-(methylsulfonyl)vinyl)benzylphosphonate

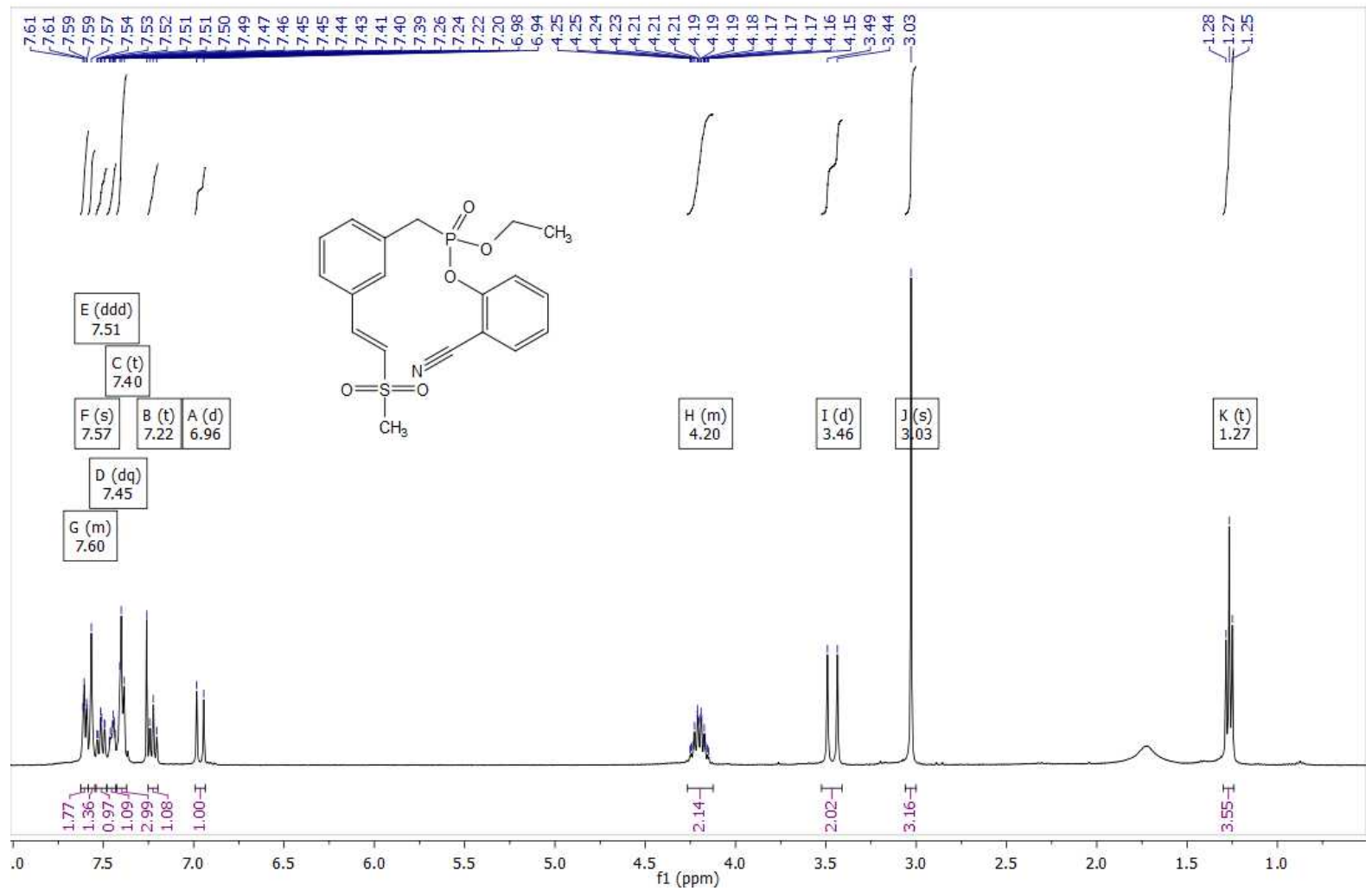

(E)-2-cyanophenyl ethyl 3-(2-(methylsulfonyl)vinyl)benzylphosphonate

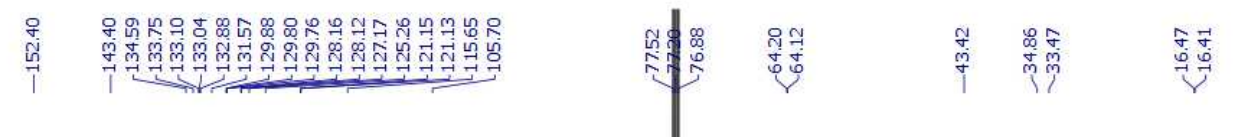<smiles>CCOP(=O)(Cc1cccc(/C=C/S(C)(=O)=O)c1)Oc1ccccc1C#N</smiles>

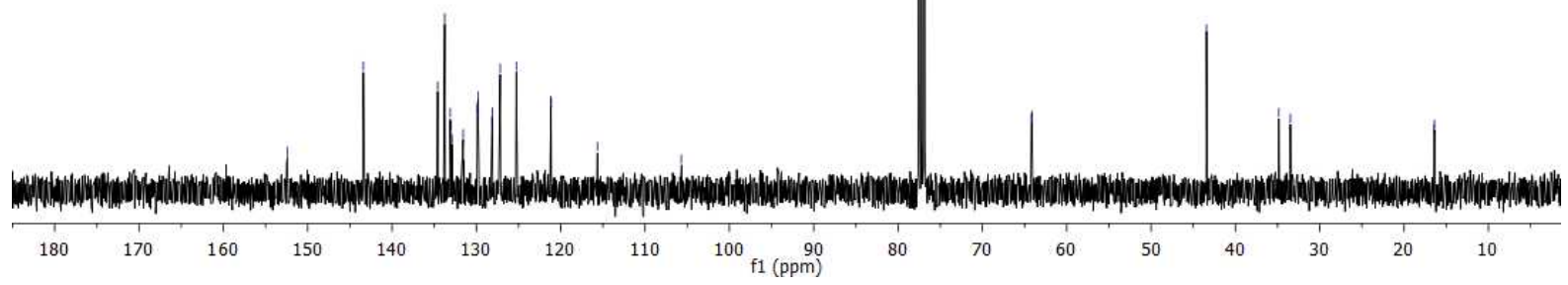


(E)-2-cyanophenyl ethyl 3-(2-(phenylsulfonyl)vinyl)benzylphosphonate (2g):

(E)-2-cyanophenyl ethyl 3-(2-(phenylsulfonyl)vinyl)benzylphosphonate

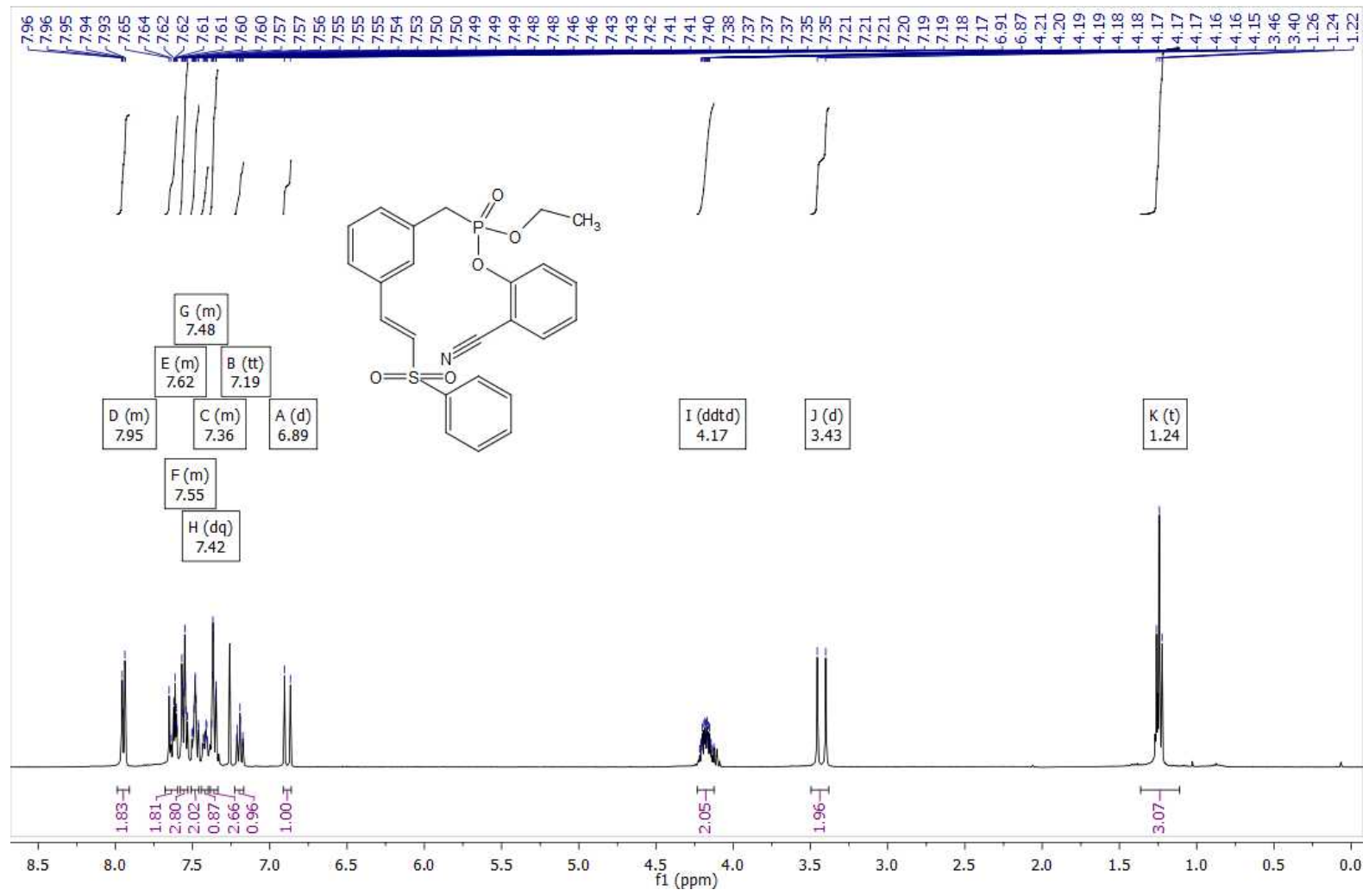

(E)-2-cyanophenyl ethyl 3-(2-(phenylsulfonyl)vinyl)benzylphosphonate
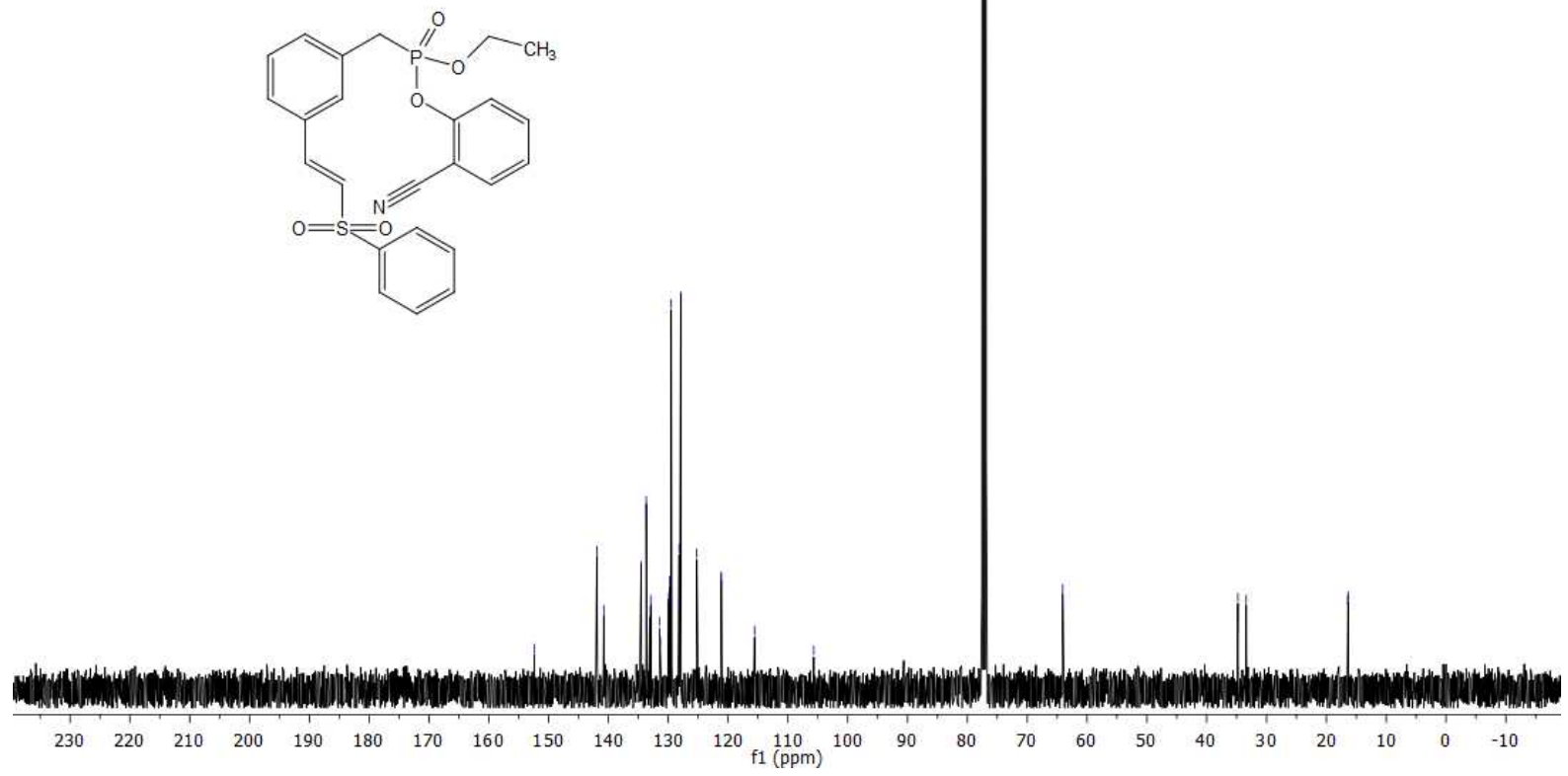
(E)-2-cyanophenyl ethyl 3-(2-(vinylsulfonyl)vinyl)benzylphosphonate (2h):

(E)-2-cyanophenyl ethyl 3-(2-(vinylsulfonyl)vinyl)benzylphosphonate

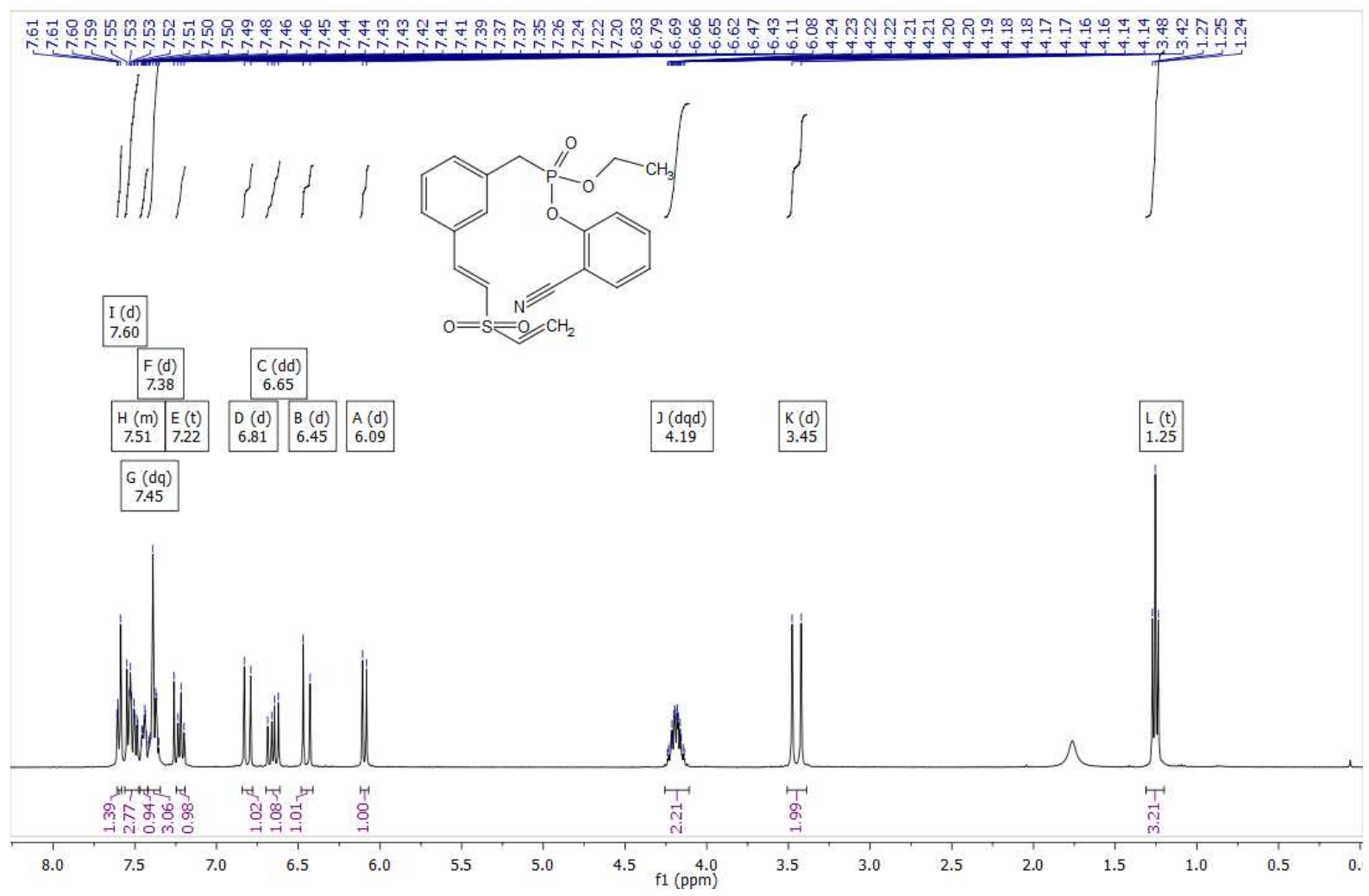

(E)-2-cyanophenyl ethyl 3-(2-(vinylsulfonyl)vinyl)benzylphosphonate

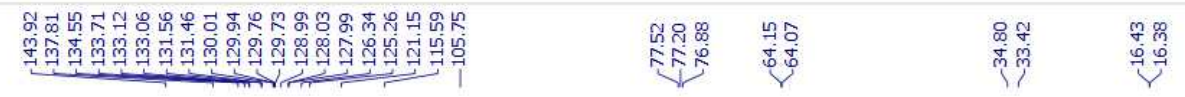
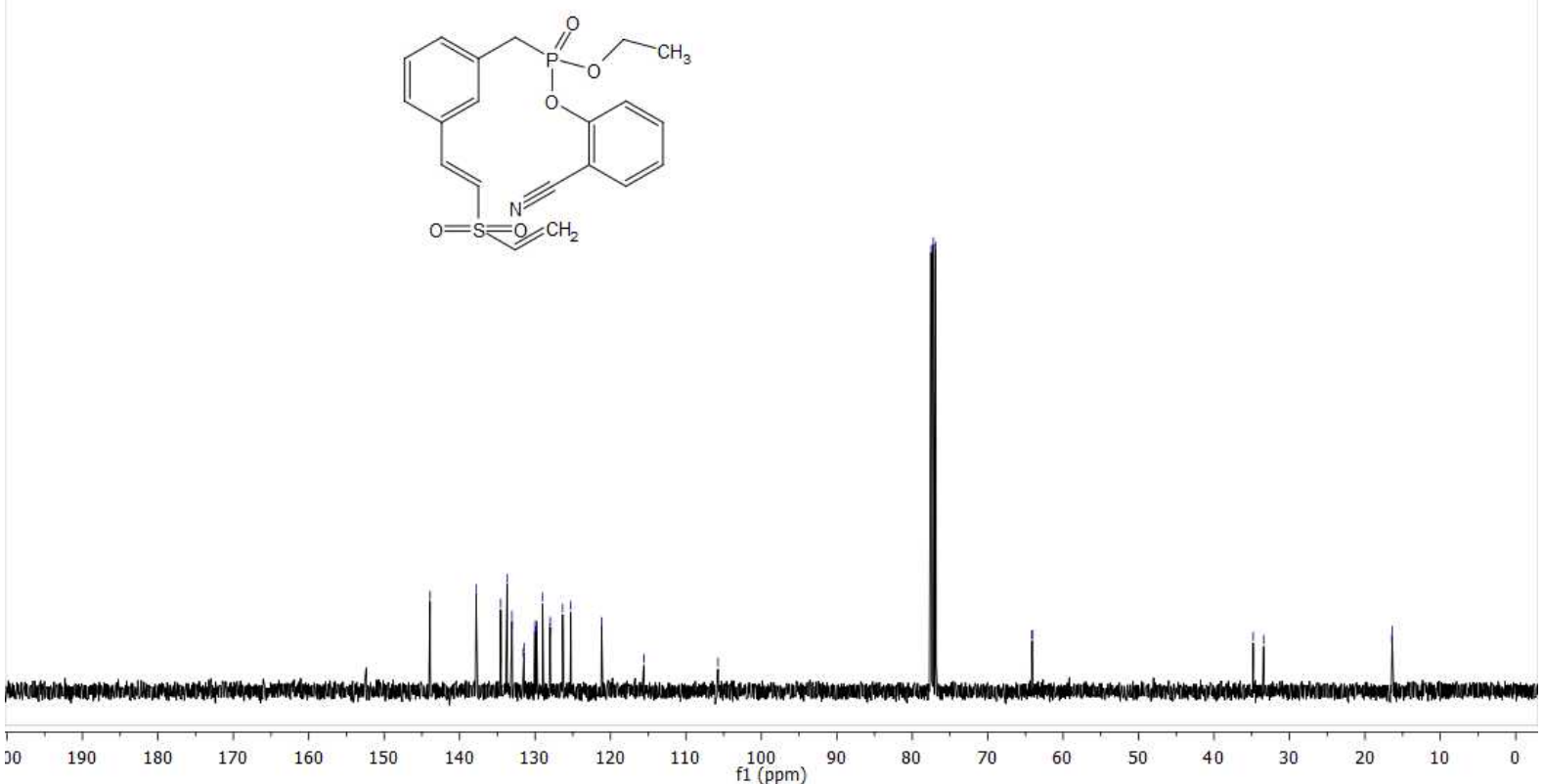
Dimethyl 2-(3-(((2-cyanophenoxy)(ethoxy)phosphoryl)methyl)phenyl)maleate (2i):

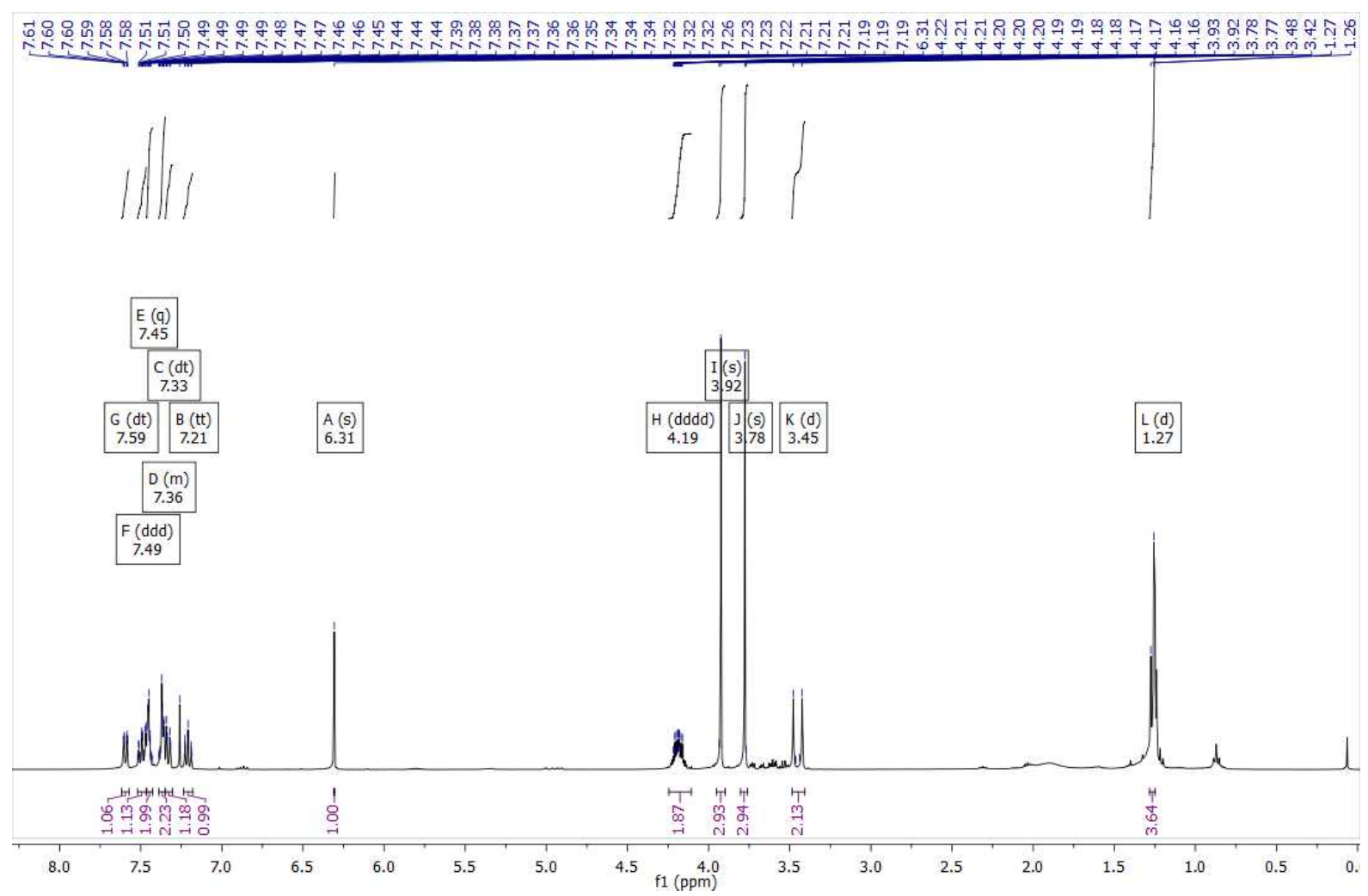

dimethyl 2-(3-(((2-cyanophenoxy)(ethoxy)phosphoryl)methyl)phenyl)maleate

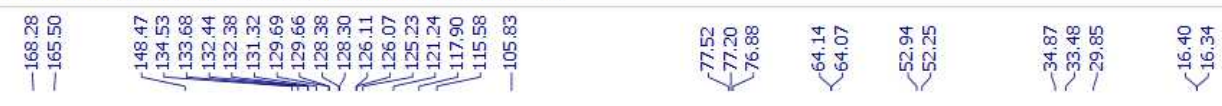<smiles>CCOP(=O)(Cc1cccc(OP(=O)(OCC)OCC)c1)OC(=CC(=O)OC)C(=O)OC</smiles>

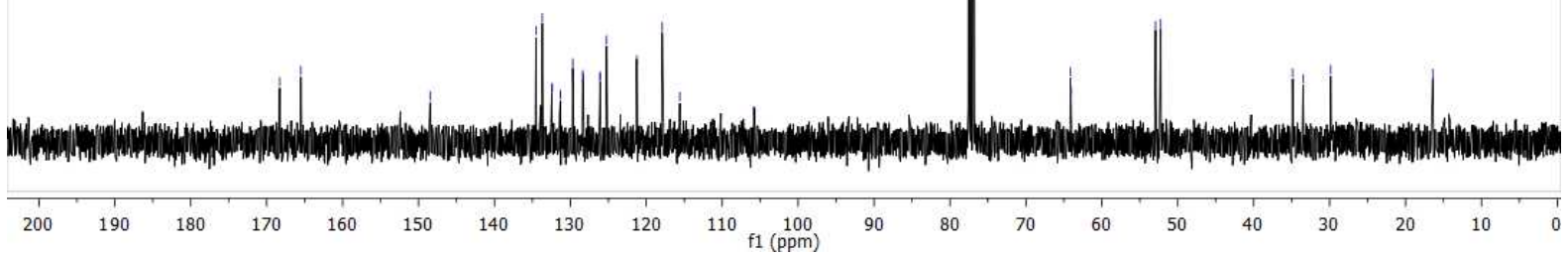


2-Cyanophenyl ethyl ((6'-acetyl-1',2',3',4'-tetrahydro-[1,1'-biphenyl]-3-yl)methyl)phosphonate $(2 \mathrm{j})$ :

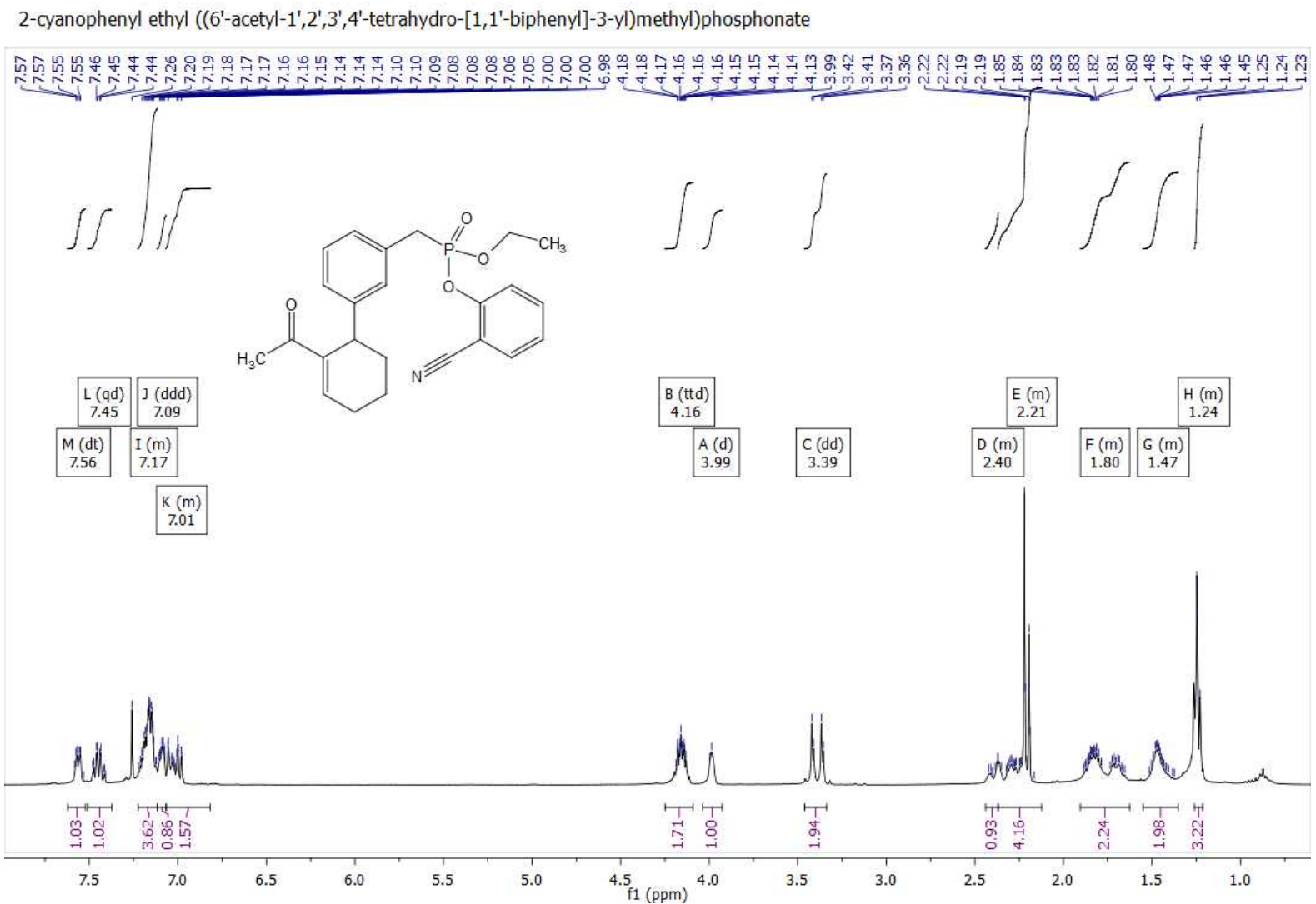

2-cyanophenyl ethyl ((6'-acetyl-1',2',3',4'-tetrahydro-[1, 1'-biphenyl]-3-yl)methyl)phosphonate

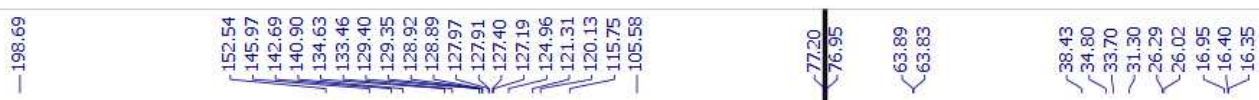<smiles>CCOP(=O)(Cc1cccc(C2CCCC=C2C(C)=O)c1)Oc1ccccc1C#N</smiles>

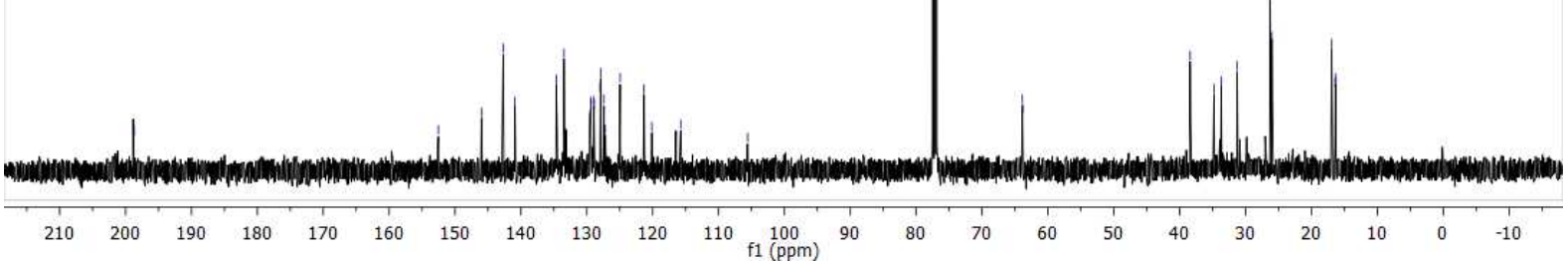


(E)-4-(3-(((2-cyanophenoxy)(ethoxy)phosphoryl)methyl)styryl)phenyl acetate (2k):

(E)-4-(3-(((2-cyanophenoxy)(ethoxy)phosphoryl)methyl)styryl)phenyl acetate

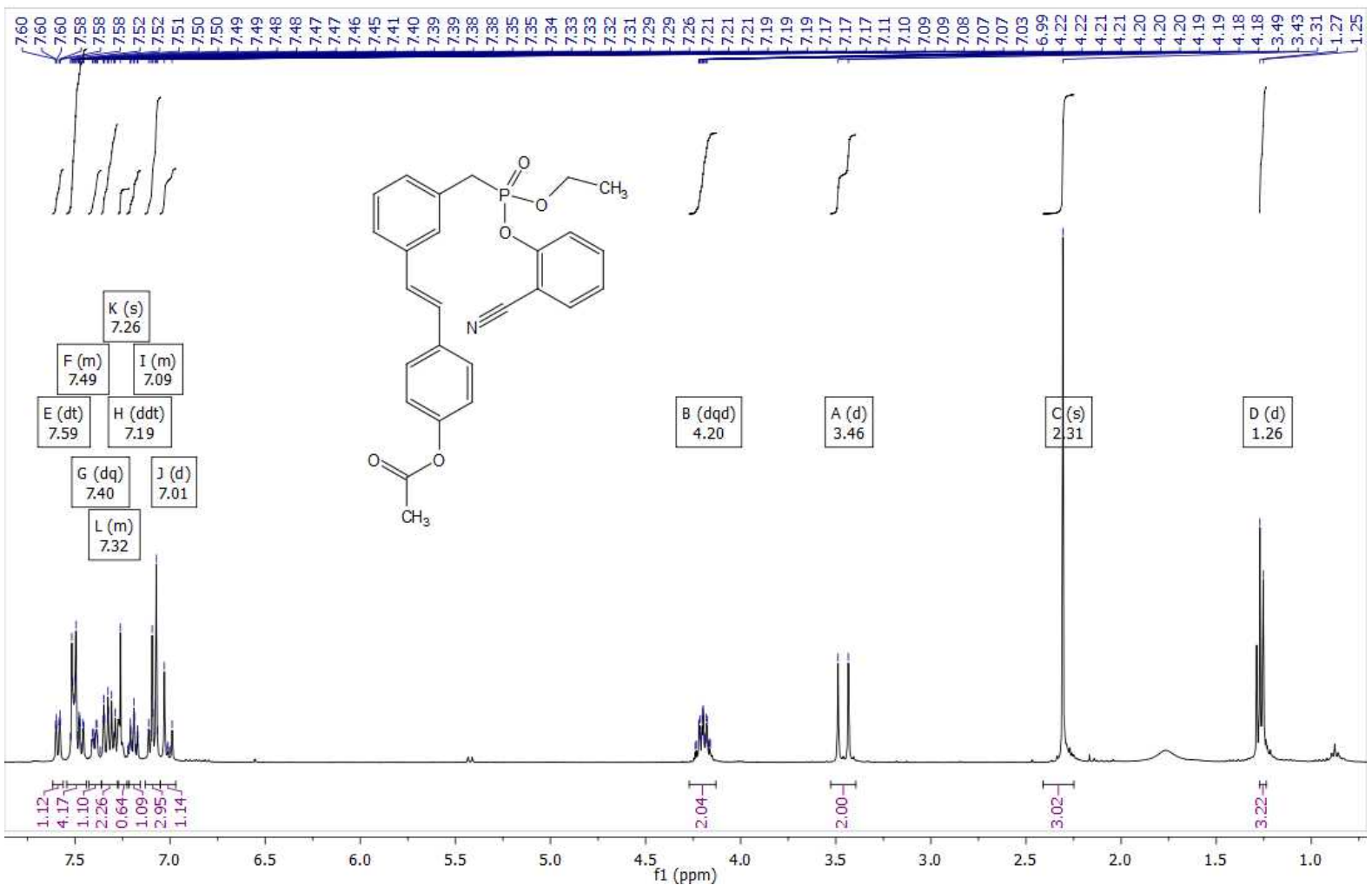

(E)-4-(3-(((2-cyanophenoxy)(ethoxy)phosphoryl)methyl)styryl)phenyl acetate

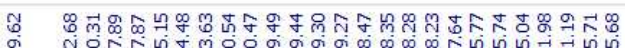

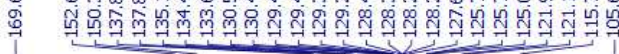

ทำ

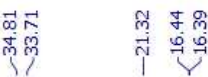

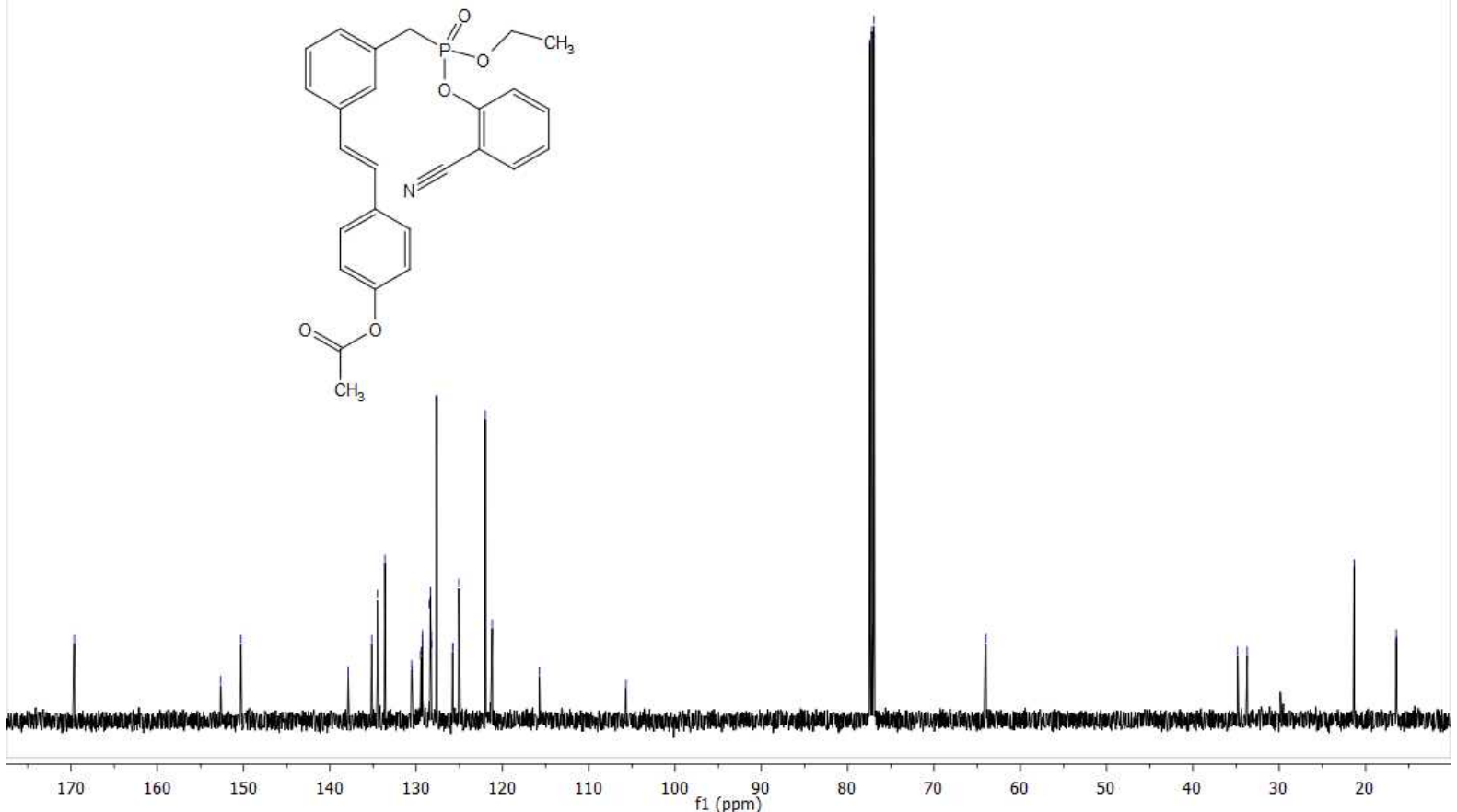


(E)-2-cyanophenyl ethyl 3-(3-nitrostyryl)benzylphosphonate (2l):

(E)-2-cyanophenyl ethyl 3-(3-nitrostyryl)benzylphosphonate

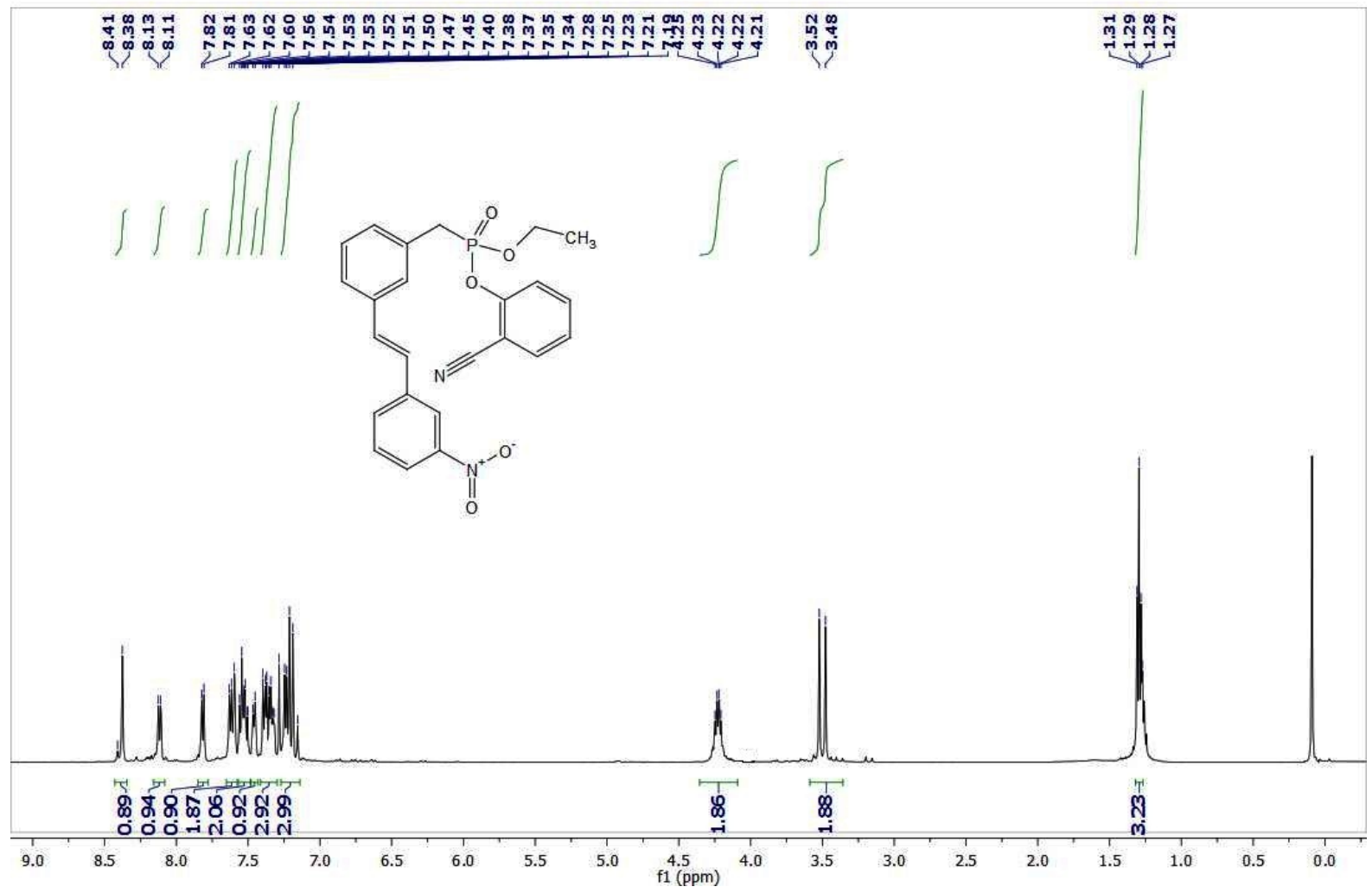

(E)-2-cyanophenyl ethyl 3-(3-nitrostyryl)benzylphosphonate

药

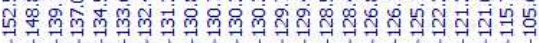

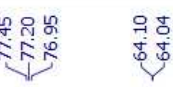

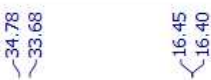
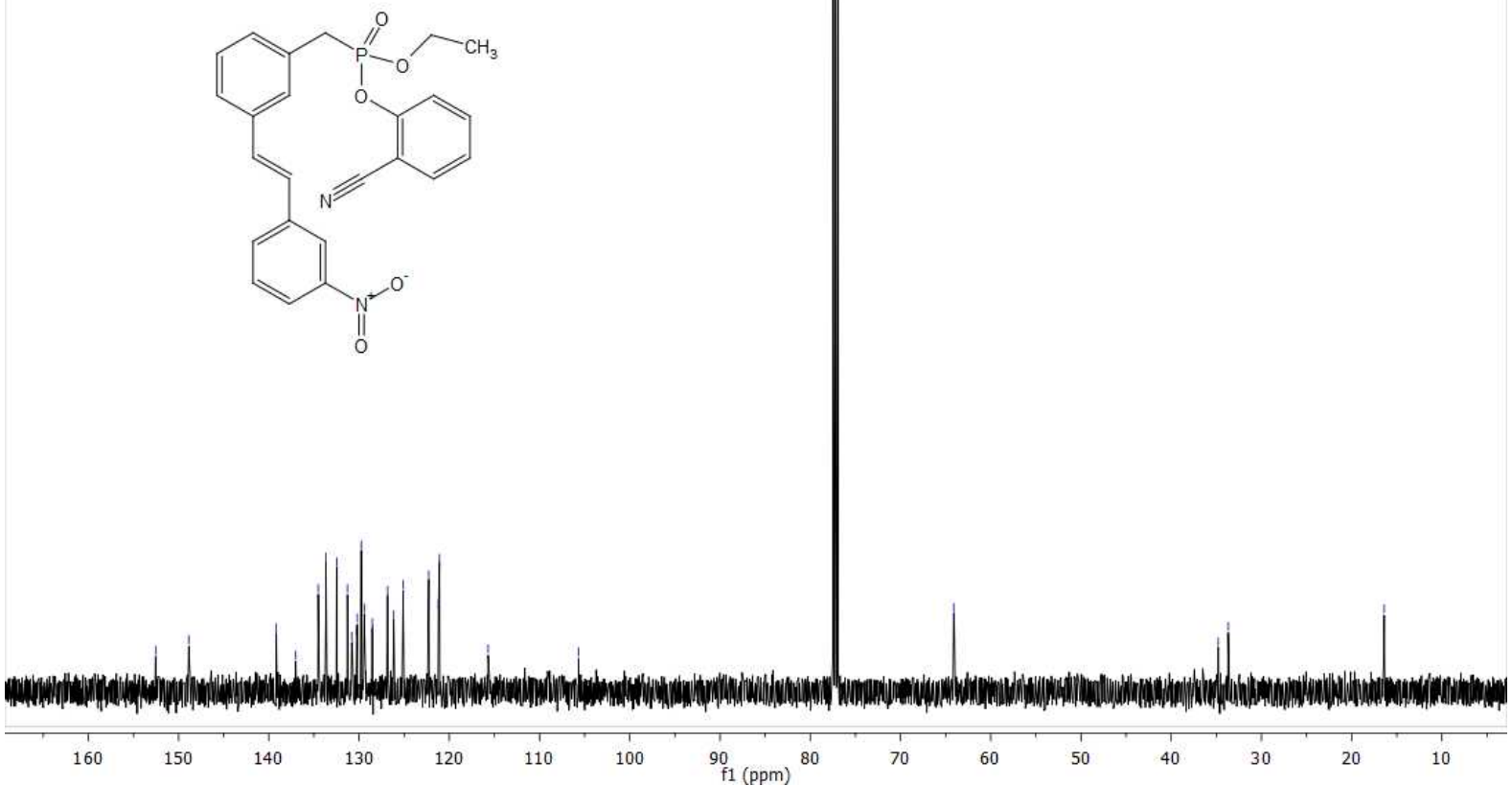
(E)-ethyl 3-(5-(((2-cyanophenoxy)(ethoxy)phosphoryl)methyl)-2-methylphenyl)acrylate (2m):

(E)-ethyl 3-(5-(((2-cyanophenoxy)(ethoxy)phosphoryl)methyl)-2-methylphenyl)acrylate

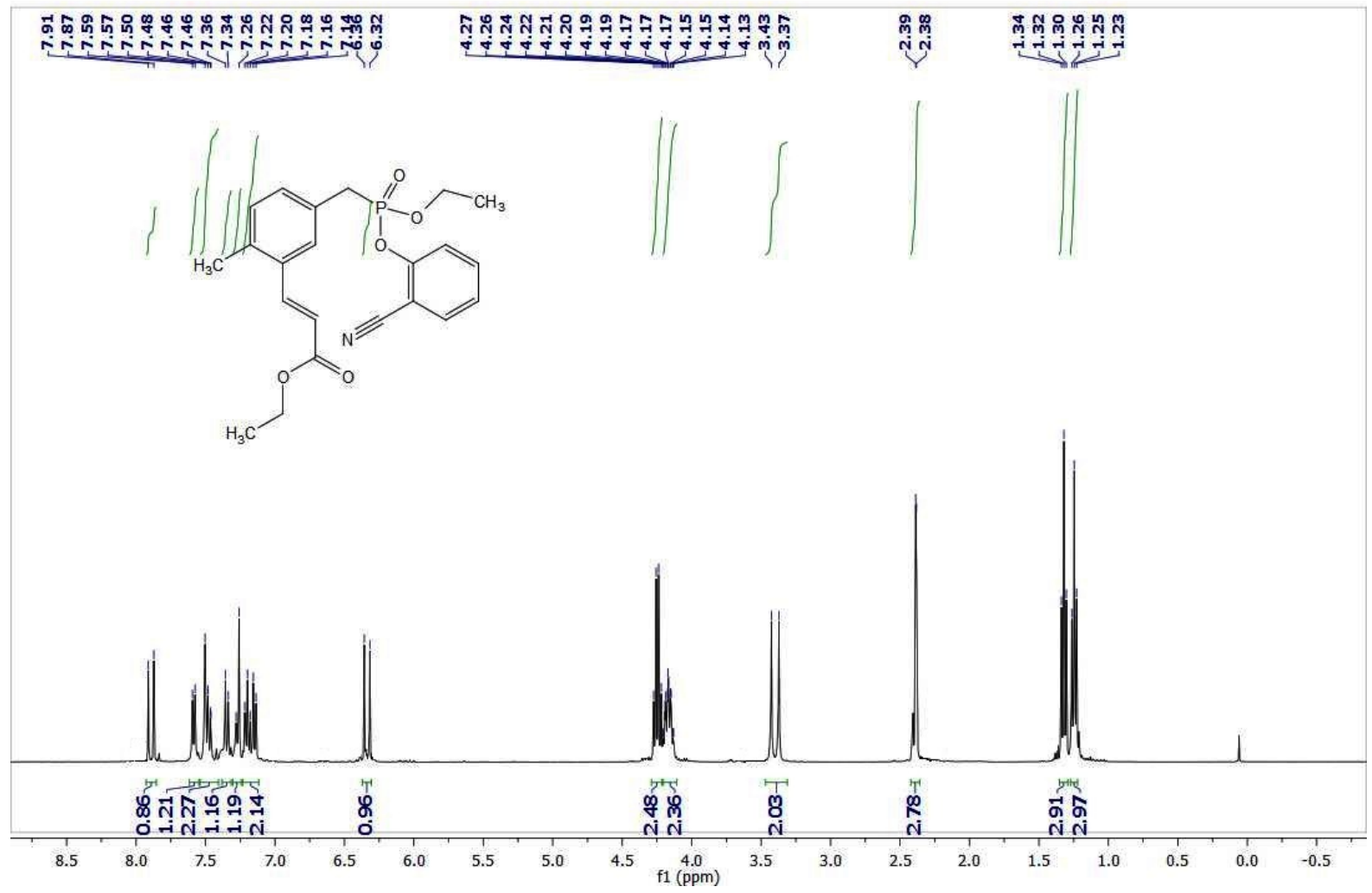

(E)-ethyl 3-(5-(((2-cyanophenoxy)(ethoxy)phosphoryl)methyl)-2-methylphenyl)acrylate

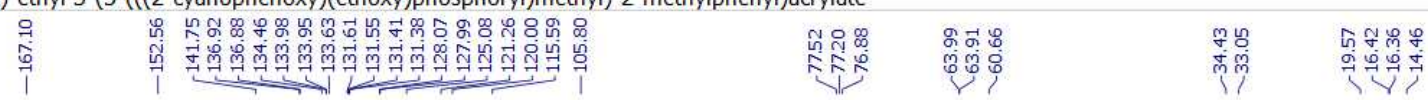
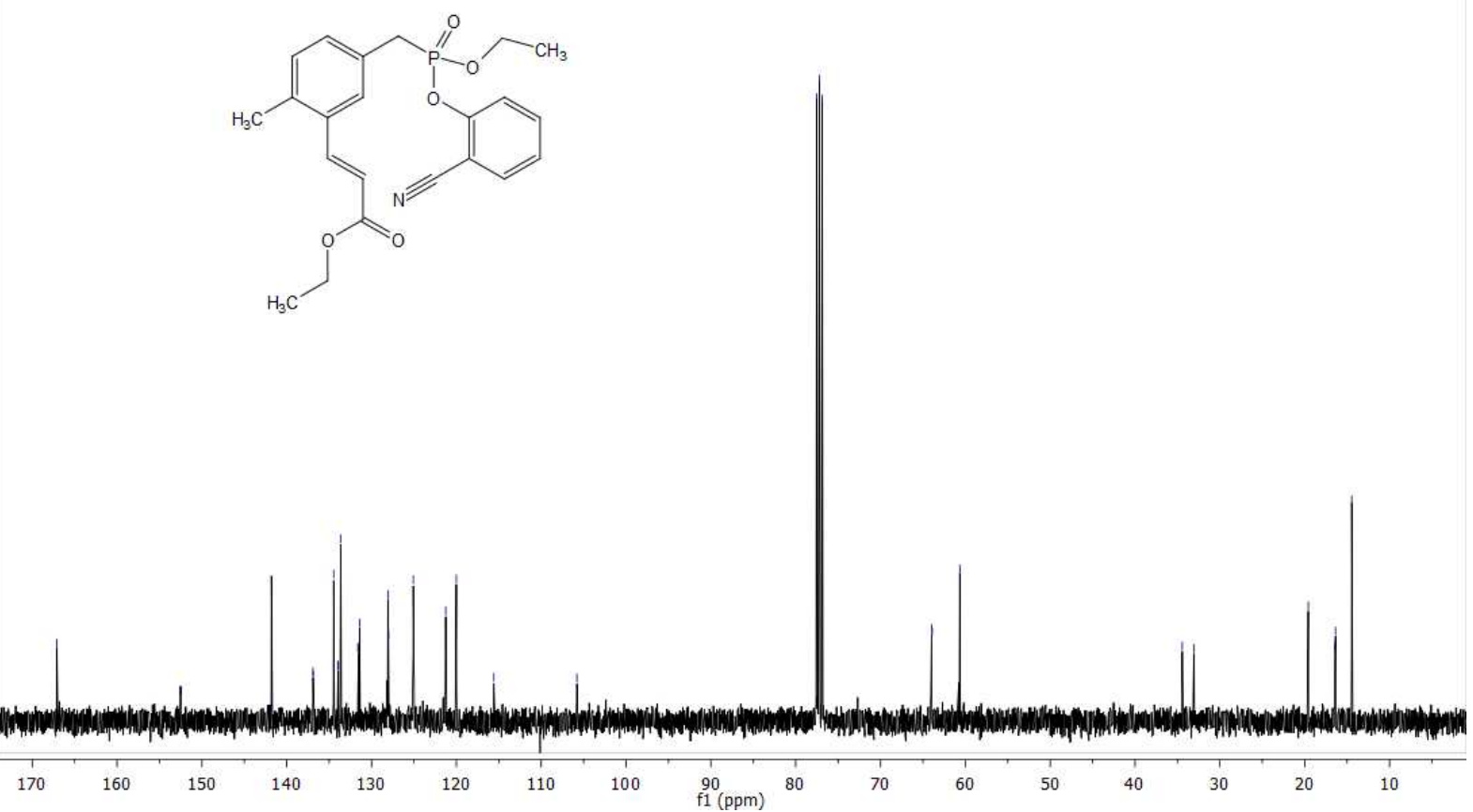
(E)-ethyl 3-(5-(((2-cyanophenoxy)(ethoxy)phosphoryl)methyl)-2-methoxyphenyl)acrylate (2n):

(E)-ethyl 3-(5-(((2-cyanophenoxy)(ethoxy)phosphoryl)methyl)-2-methoxyphenyl)acrylate

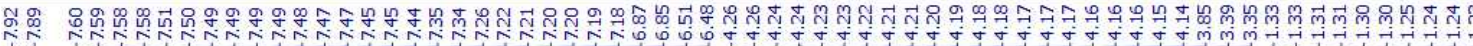

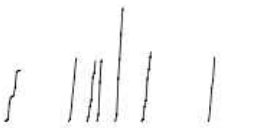<smiles>[O-][I+]O</smiles><smiles>C=CCCCCCCOc1cccc(OC)c1</smiles>
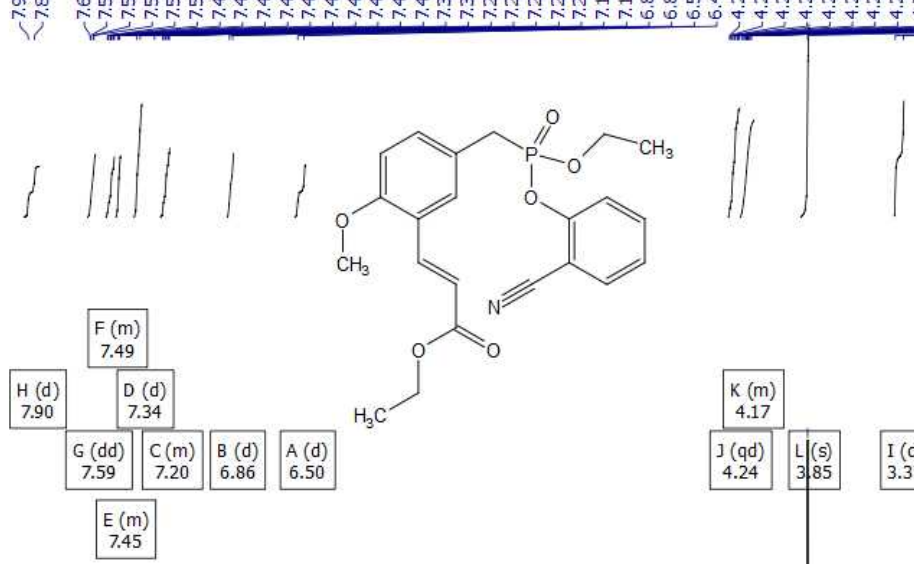

$\mathrm{CH}_{3}$<smiles>C=C(C#N)/C=C\CCC</smiles>
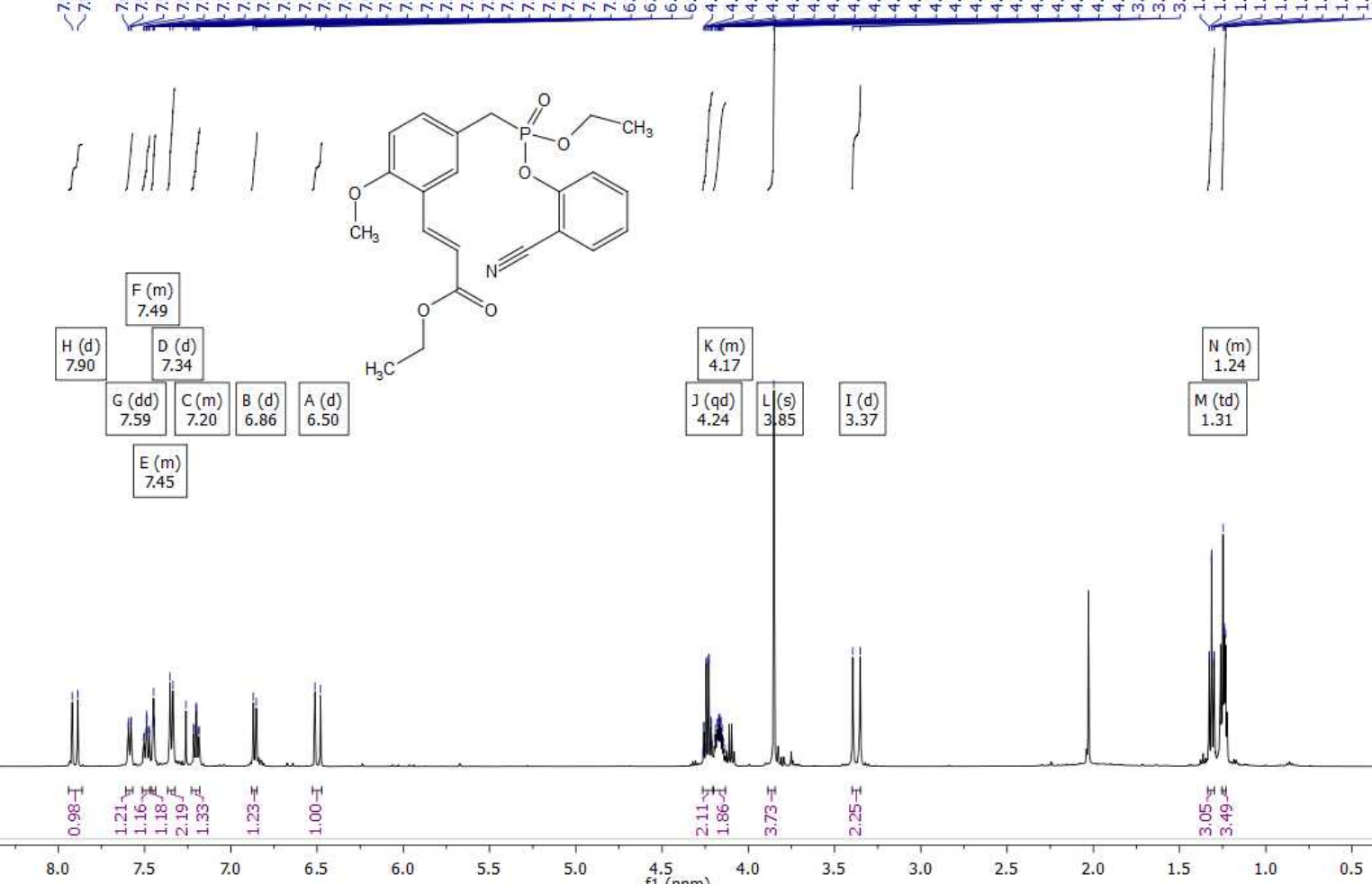

g

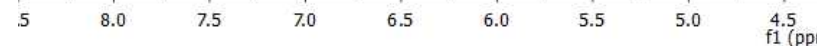

(E)-ethyl 3-(5-(((2-cyanophenoxy)(ethoxy)phosphoryl)methyl)-2-methoxyphenyl)acrylate

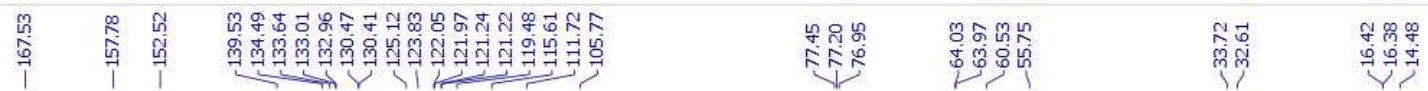<smiles>CCOC(=O)/C=C/c1cc(CP(=O)(OCC)Oc2ccccc2C#N)ccc1OC</smiles>

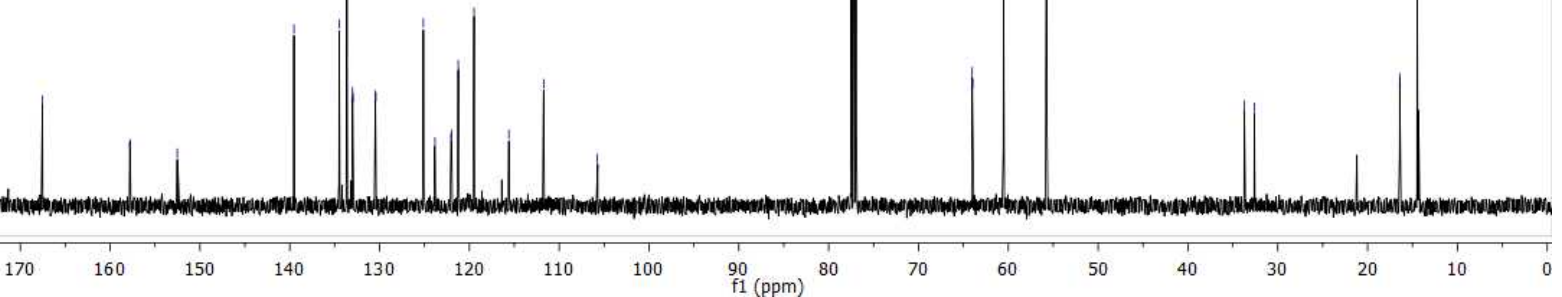


31P NMR:

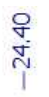

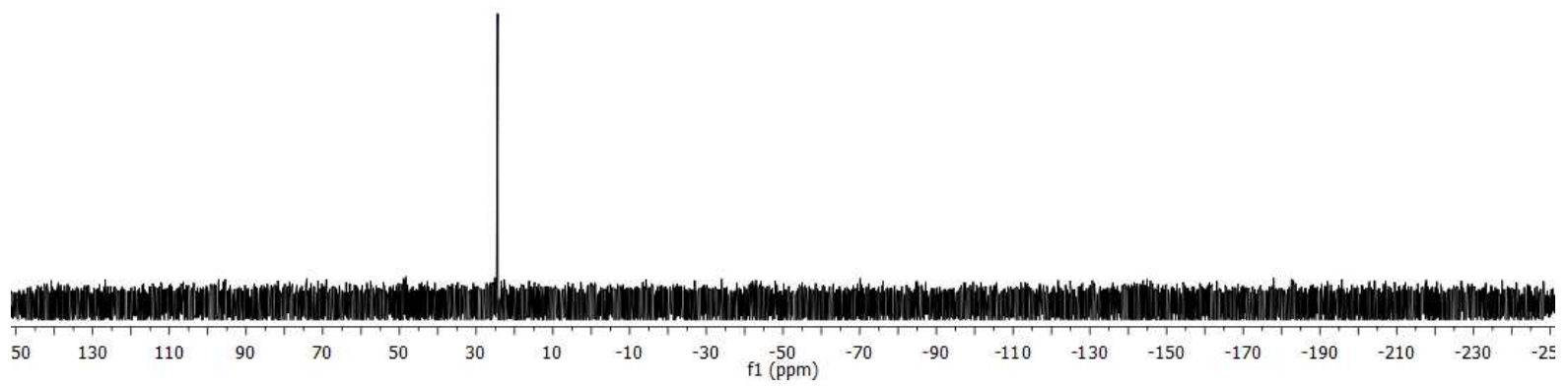


(E)-ethyl 3-(2-chloro-5-(((2-cyanophenoxy)(ethoxy)phosphoryl)methyl)phenyl)acrylate (2p):

(E)-ethyl 3-(2-chloro-5-(((2-cyanophenoxy)(ethoxy)phosphoryl)methyl)phenyl)acrylate

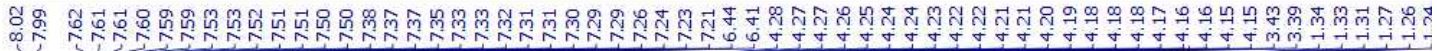
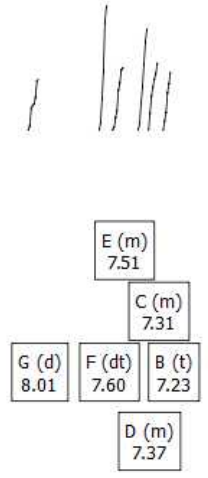<smiles>C1CCCCC1</smiles><smiles></smiles>

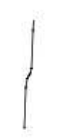

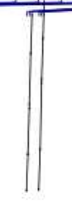

$3(d)$

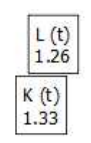

(m)

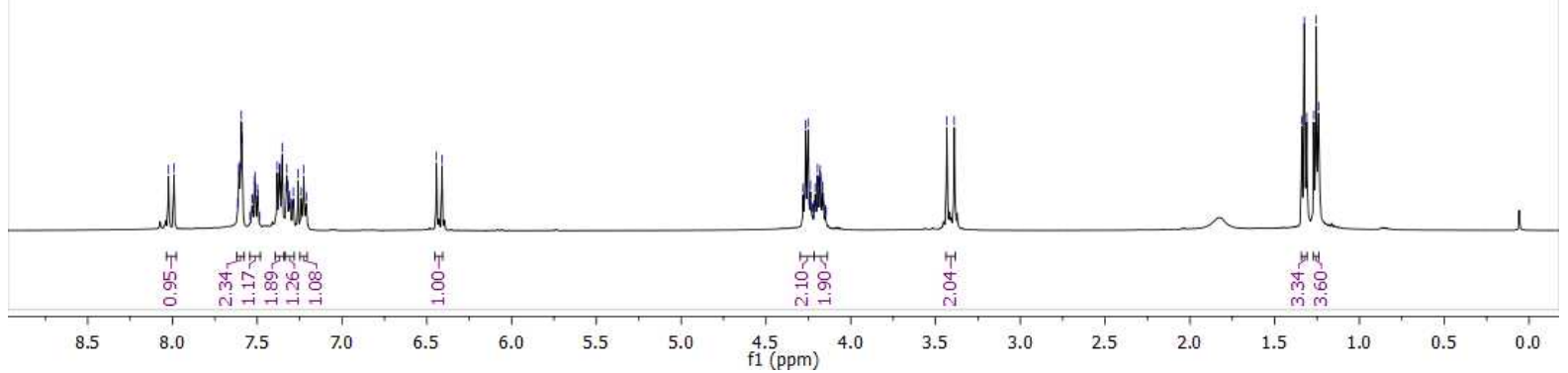

(E)-ethyl 3-(2-chloro-5-(((2-cyanophenoxy)(ethoxy)phosphoryl)methyl)phenyl)acrylate

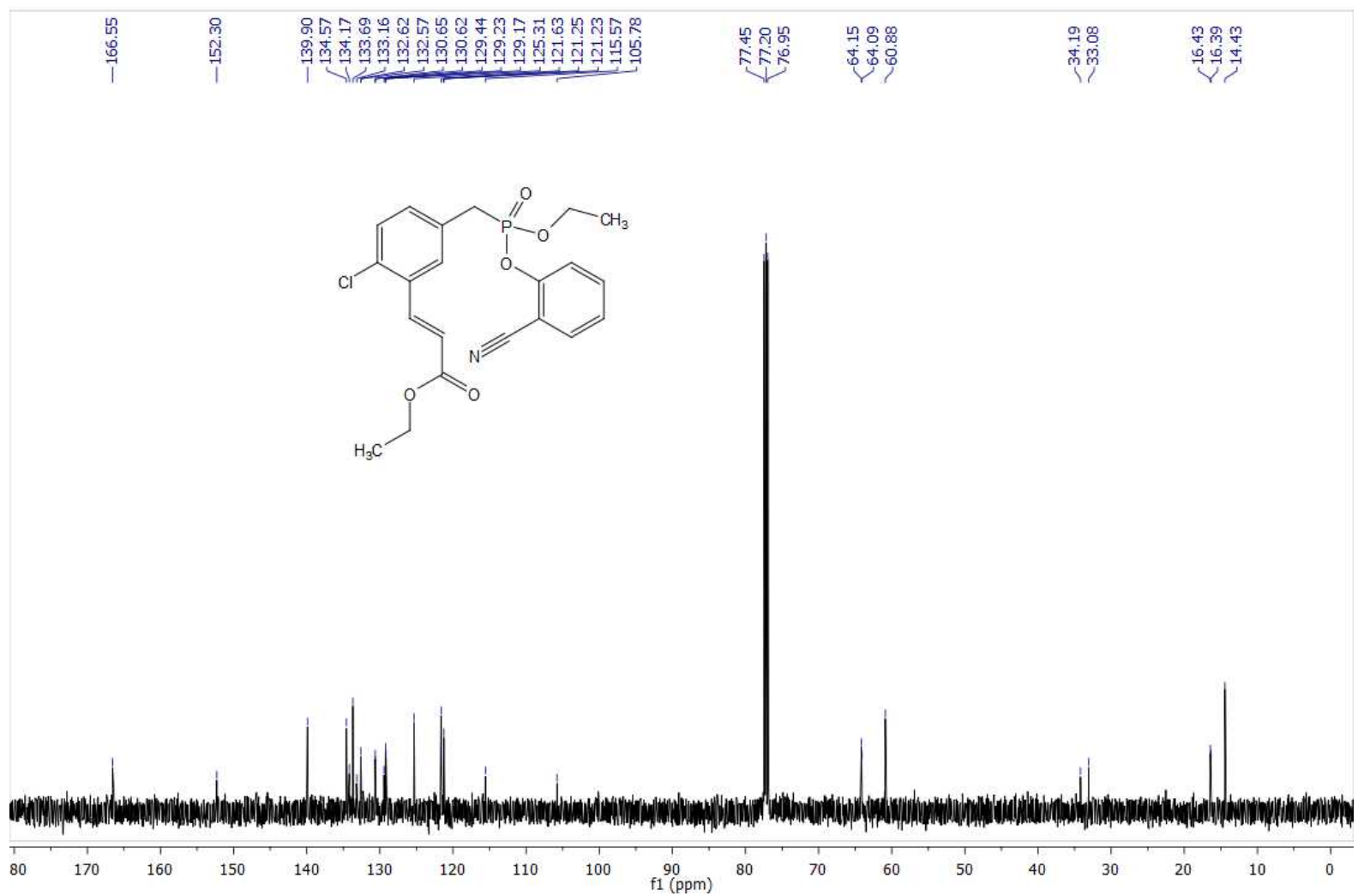


(E)-ethyl 3-(2-bromo-5-(((2-cyanophenoxy)(ethoxy)phosphoryl)methyl)phenyl)acrylate (2q):

(E)-ethyl 3-(2-bromo-5-(((2-cyanophenoxy)(ethoxy)phosphoryl)methyl)phenyl)acrylate

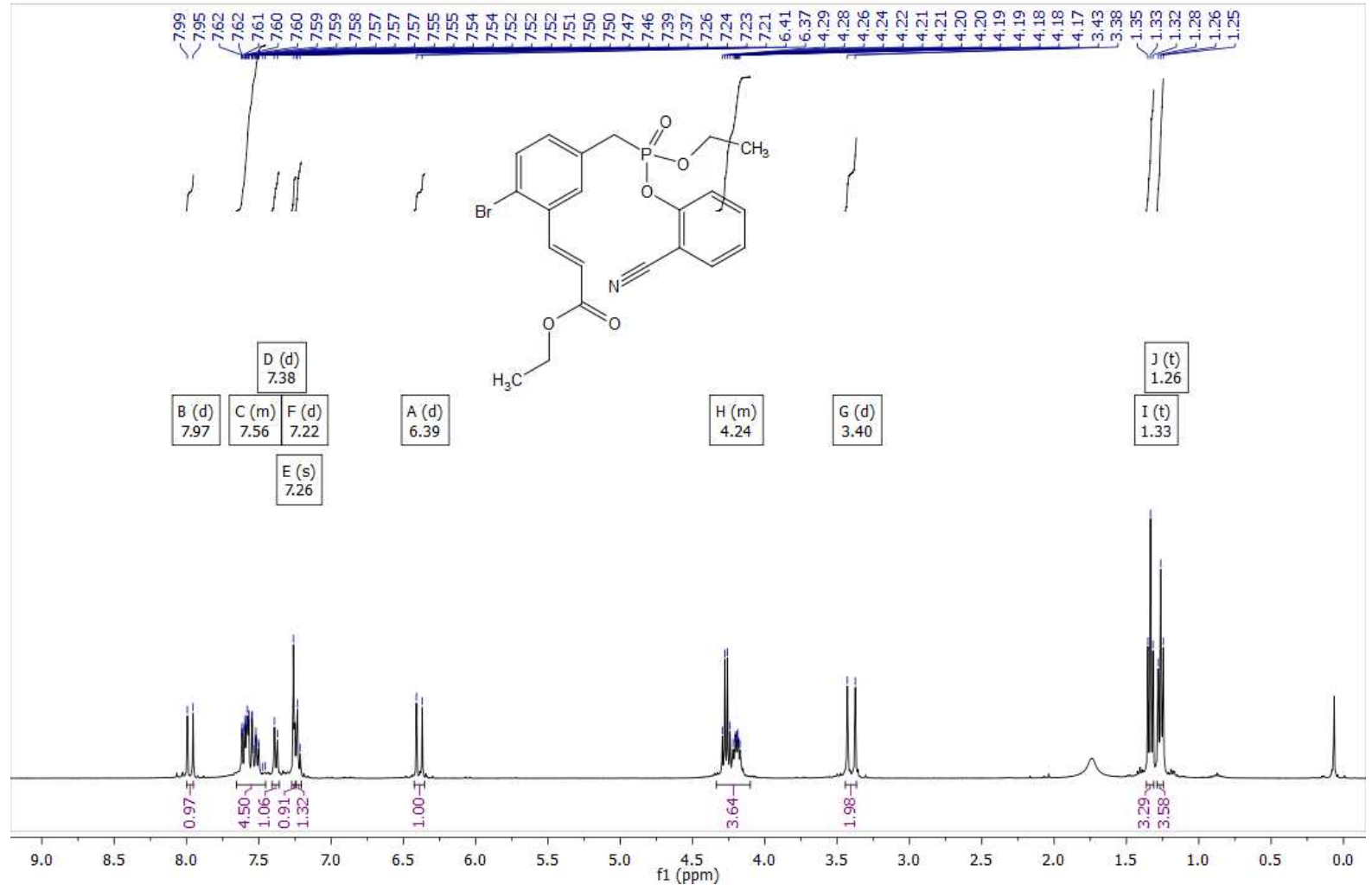

(E)-ethyl 3-(2-bromo-5-(((2-cyanophenoxy)(ethoxy)phosphoryl)methyl)phenyl)acrylate

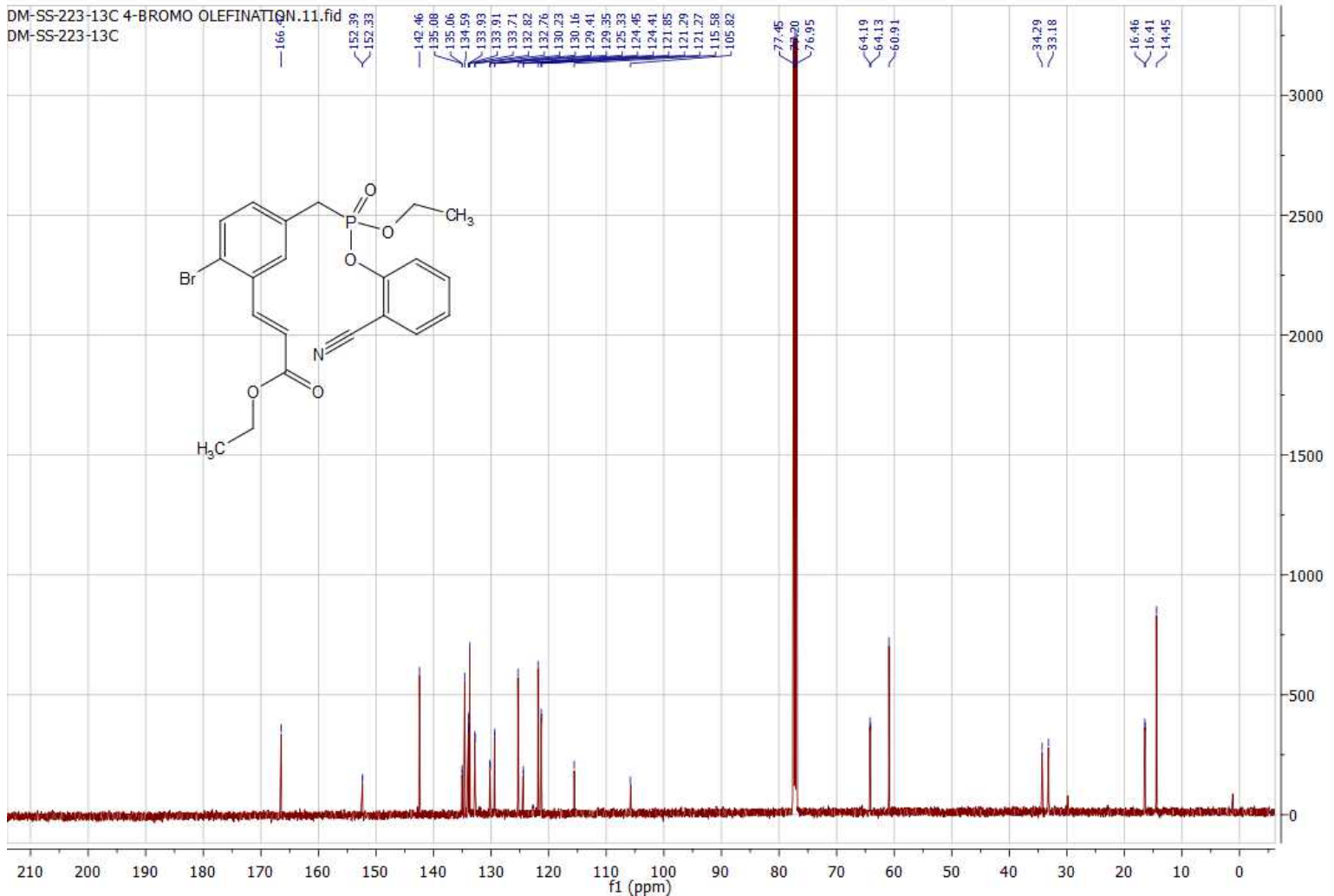


(E)-ethyl 3-(3-(((2-cyanophenoxy)(ethoxy)phosphoryl)methyl)-2,5-difluorophenyl)acrylate (2r):

(E)-ethyl 3-(3-(((2-cyanophenoxy)(ethoxy)phosphoryl)methyl)-2,5-difluorophenyl)acrylate

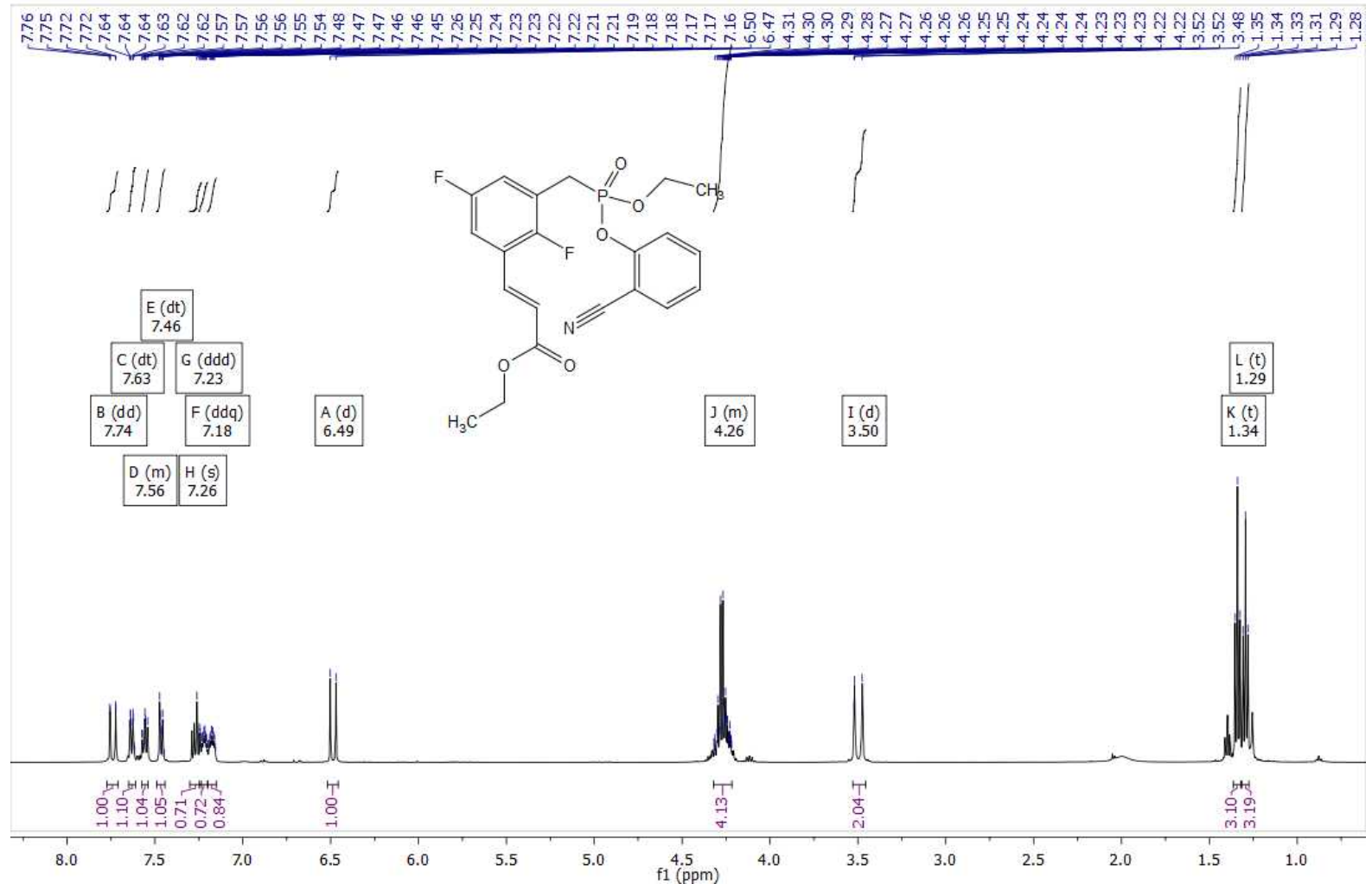

(E)-ethyl 3-(3-(((2-cyanophenoxy)(ethoxy)phosphoryl)methyl)-2,5-difluorophenyl)acrylate

\begin{tabular}{|c|c|c|}
\hline 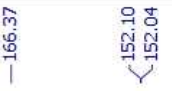 & 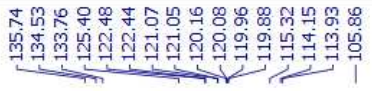 & 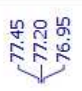 \\
\hline
\end{tabular}<smiles>CCOC(=O)/C=C/c1cc(F)cc(CP(=O)(OCC)Oc2ccccc2C#N)c1F</smiles>

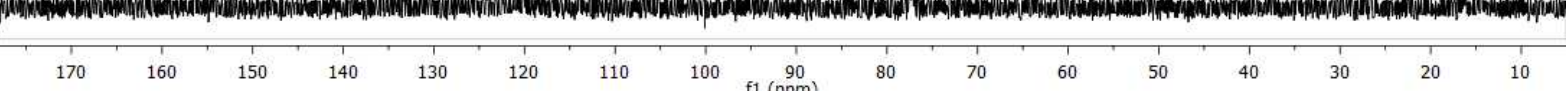


(E)-ethyl 3-(4-bromo-3-(((2-cyanophenoxy)(ethoxy)phosphoryl)methyl)phenyl)acrylate (2s):

(E)-ethyl 3-(4-bromo-3-(((2-cyanophenoxy)(ethoxy)phosphoryl)methyl)phenyl)acrylate

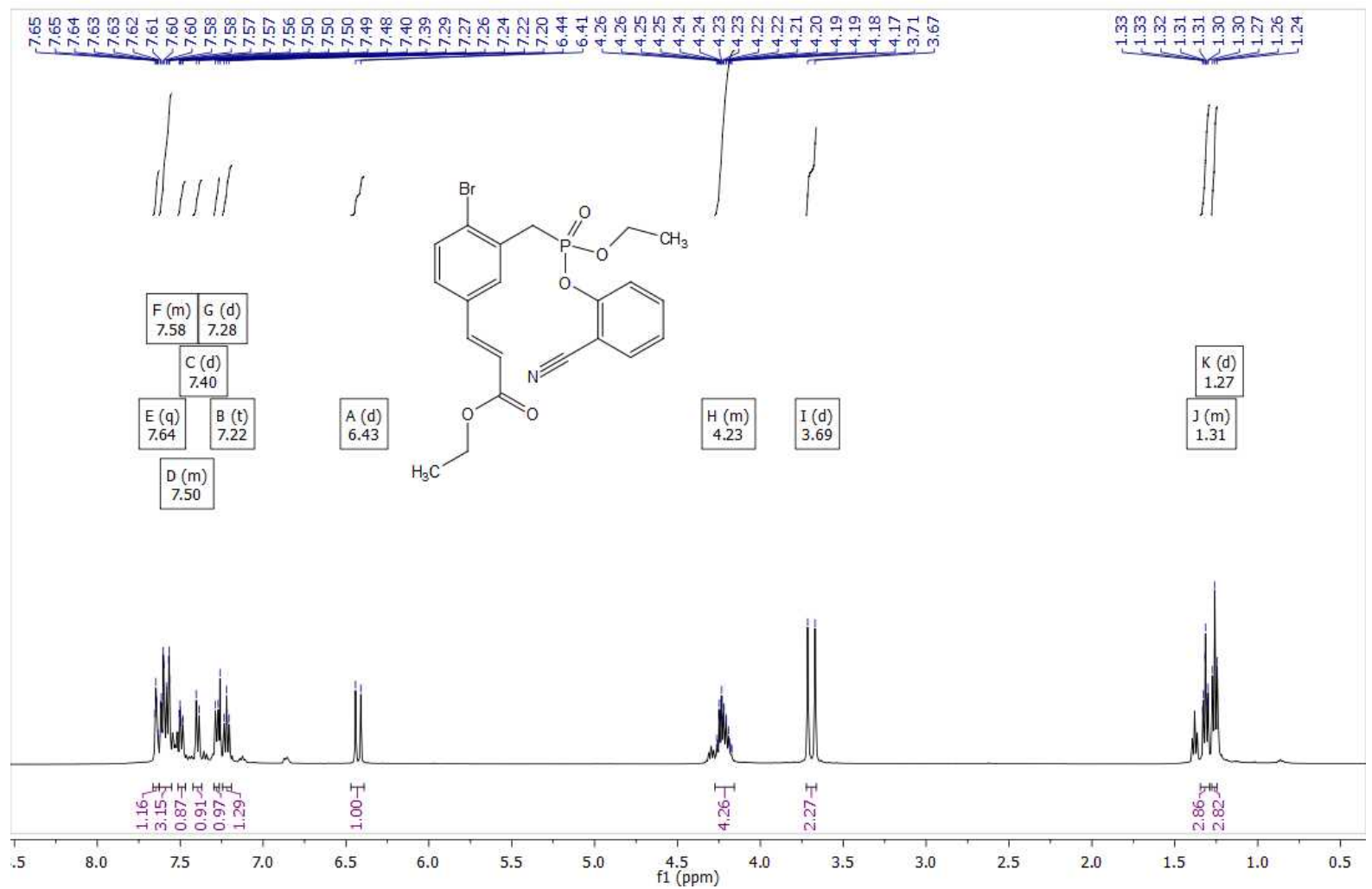

(E)-ethyl 3-(4-bromo-3-(((2-cyanophenoxy)(ethoxy)phosphoryl)methyl)phenyl)acrylate

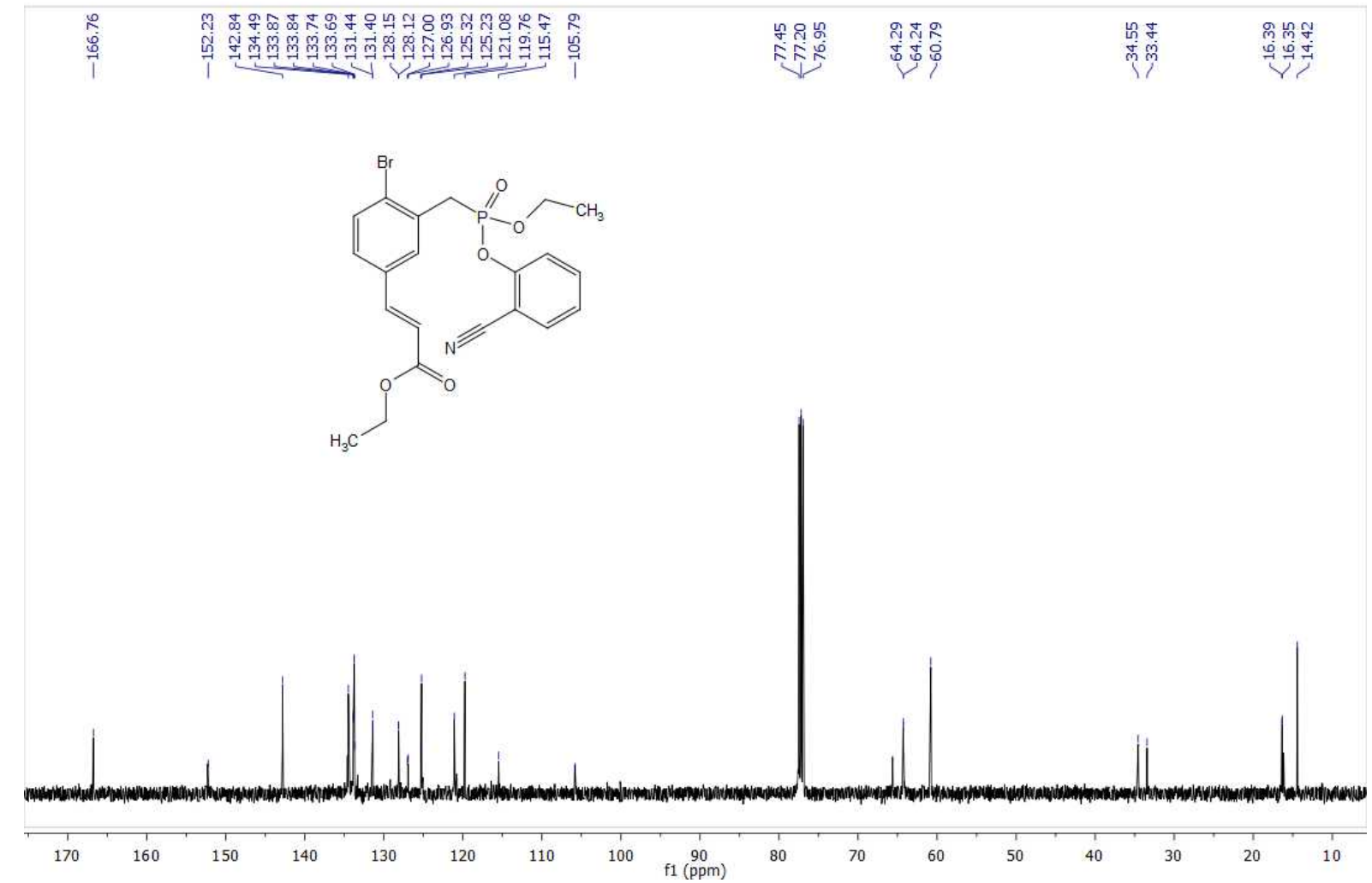


(E)-ethyl 3-(3-(((2-cyanophenoxy)(ethoxy)phosphoryl)methyl)-4-methylphenyl)acrylates (2t):

(E)-ethyl 3-(3-(((2-cyanophenoxy)(ethoxy)phosphoryl)methyl)-4-methylphenyl)acrylate

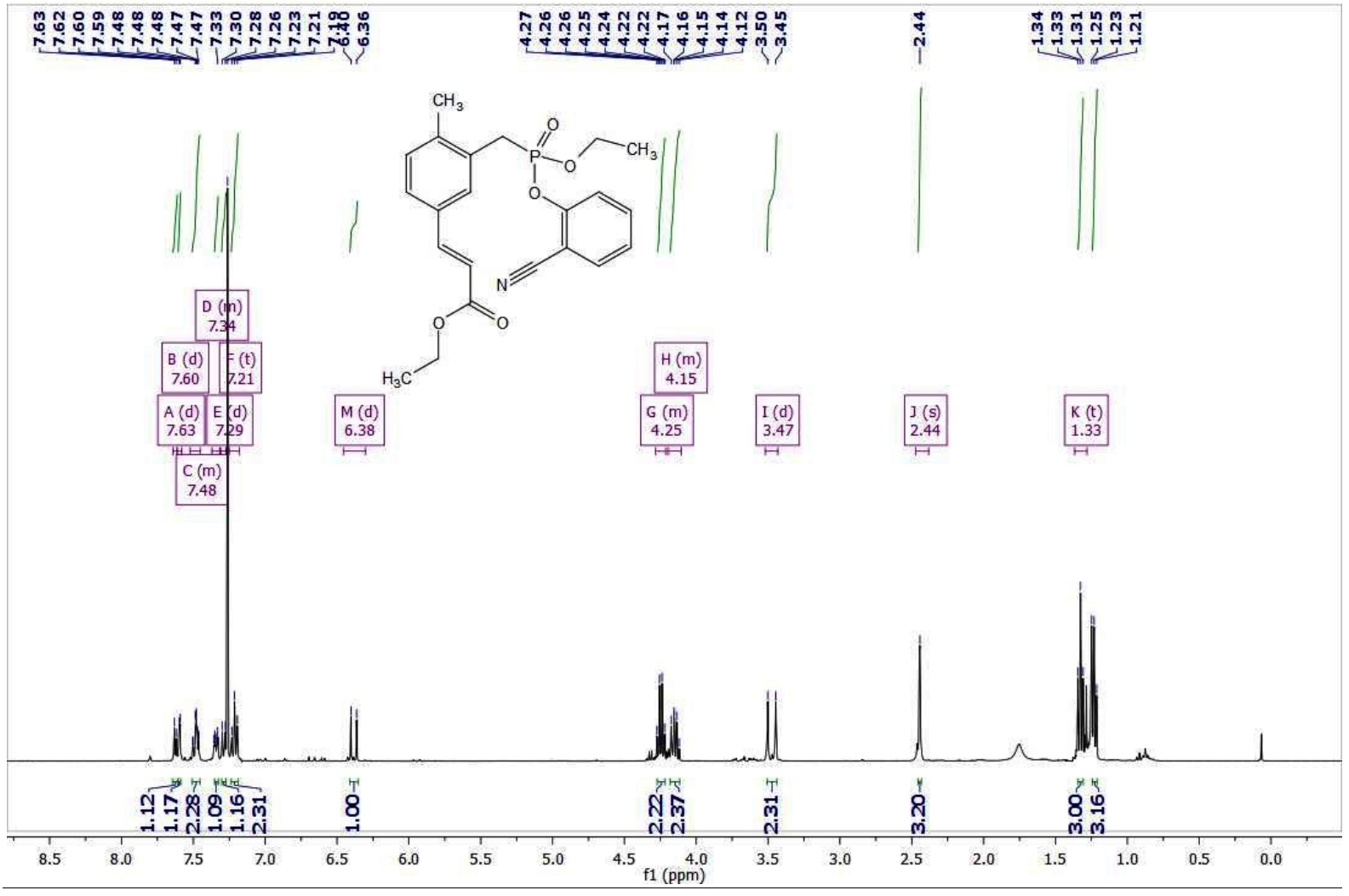

(E)-ethyl 3-(3-(((2-cyanophenoxy)(ethoxy)phosphoryl)methyl)-4-methylphenyl)acrylate

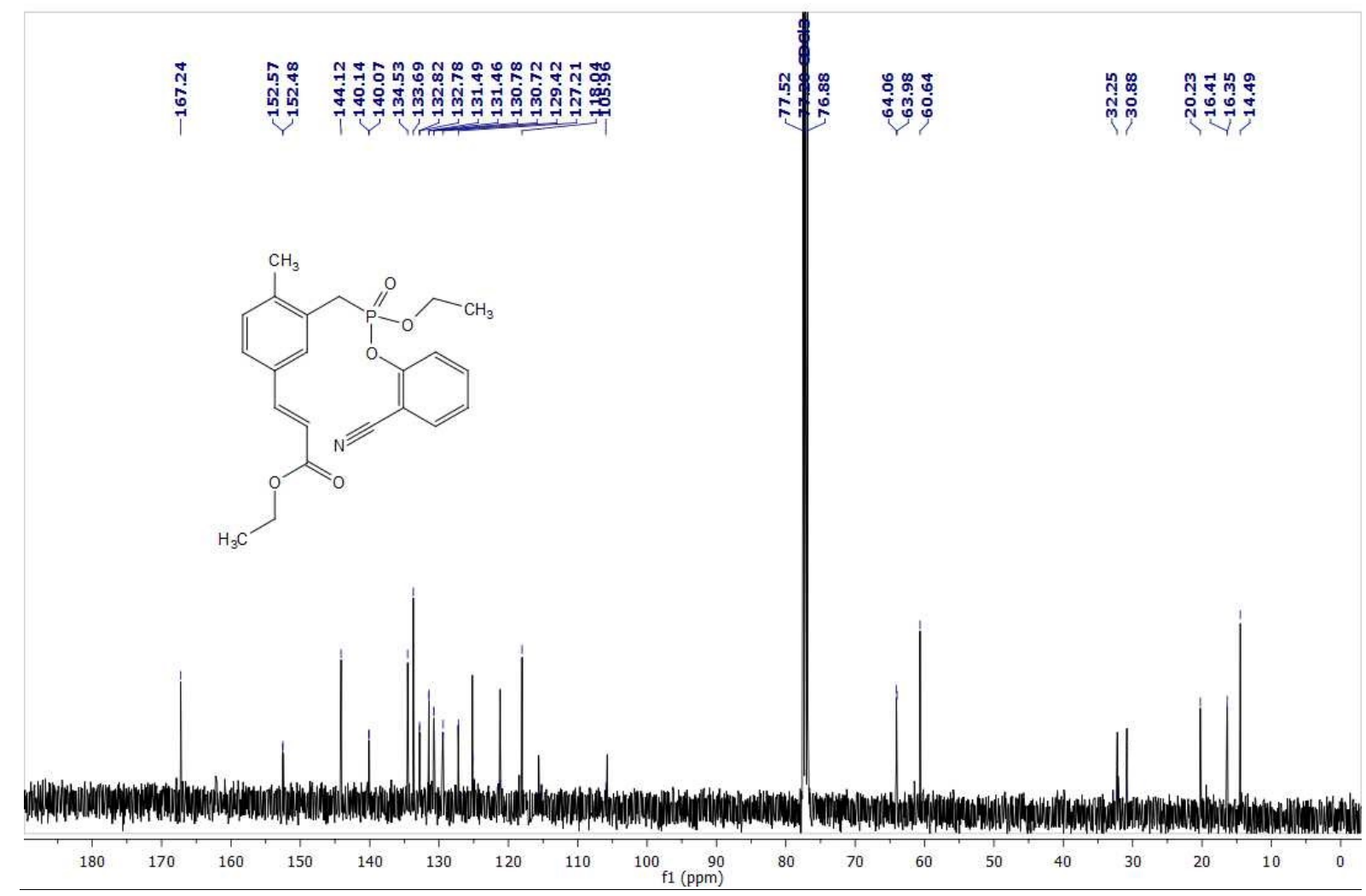


(E)-ethyl 3-(3-bromo-5-(((2-cyanophenoxy)(ethoxy)phosphoryl)methyl)phenyl)acrylate (2u):

(E)-ethyl 3-(3-bromo-5-(((2-cyanophenoxy)(ethoxy)phosphoryl)methyl)phenyl)acrylate

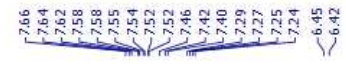

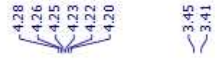
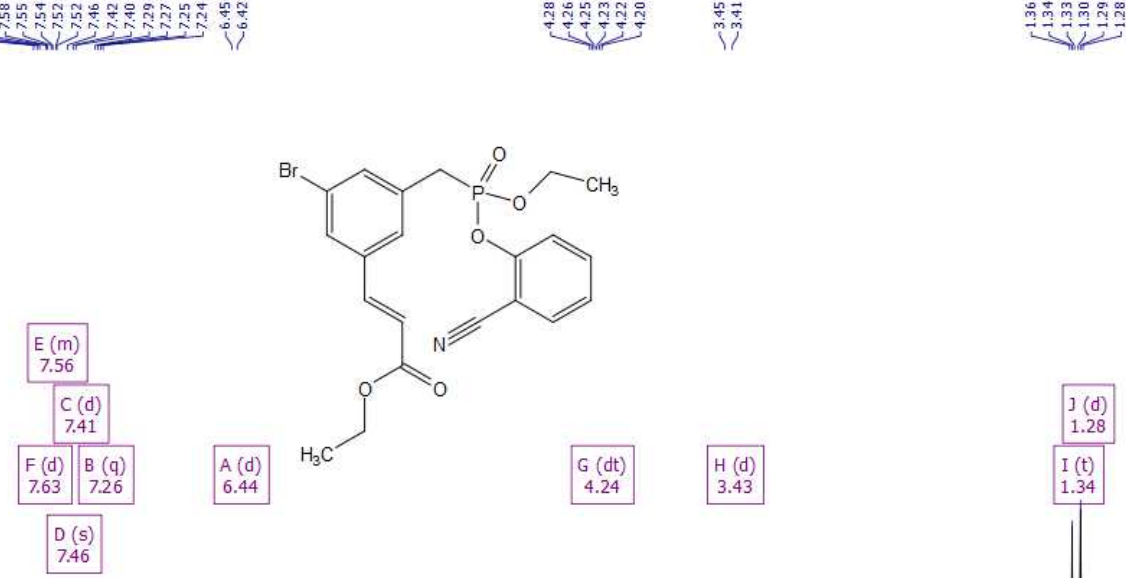

(d)
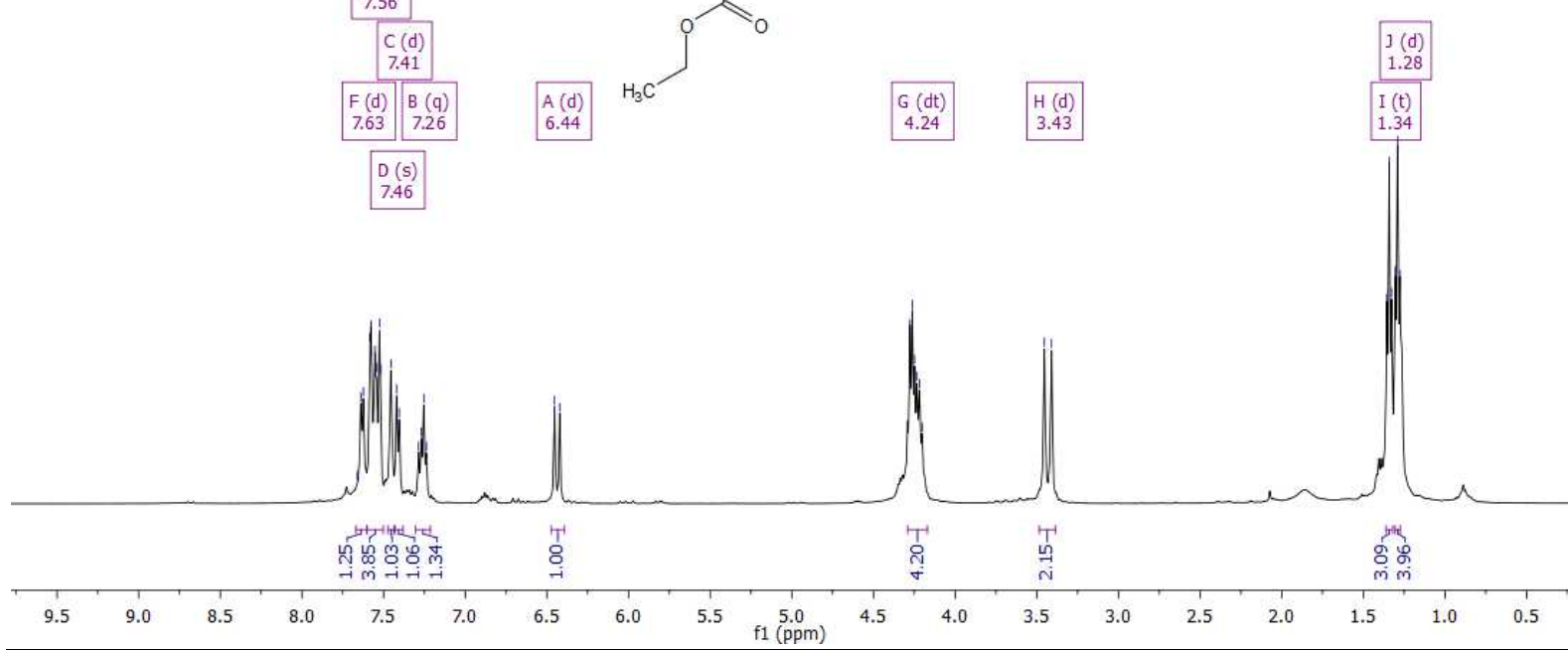

(E)-ethyl 3-(3-bromo-5-(((2-cyanophenoxy)(ethoxy)phosphoryl)methyl)phenyl)acrylate

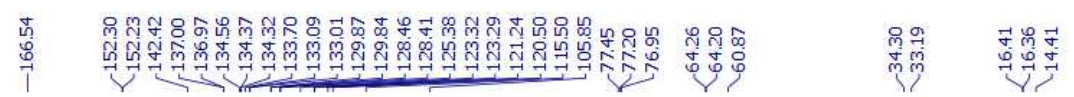<smiles>CCOC(=O)/C=C/c1cc(Br)cc(CP(=O)(OCC)Oc2ccccc2C#N)c1</smiles>

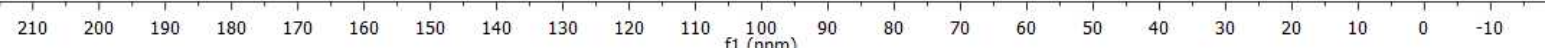


31P NMR:

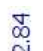

$\stackrel{\text { N }}{1}$

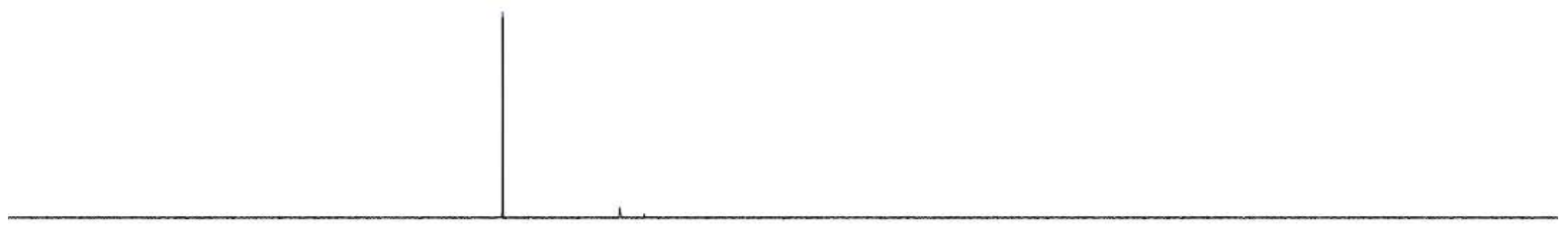

\begin{tabular}{lllllllllllllllllllllllllllllllll}
\hline 50 & 130 & 110 & 90 & 70 & 50 & 30 & 10 & -10 & -30 & $\left.\begin{array}{c}-50 \\
\mathrm{f1}\end{array} \mathrm{ppm}\right)$ & -70 & -90 & -110 & -130 & -150 & -170 & -190 & -210 & -230 & -25 \\
\hline
\end{tabular}


(E)-ethyl 3-(3-(((2-cyanophenoxy)(ethoxy)phosphoryl)methyl)-5-methylphenyl)acrylates (2v):

(E)-ethyl 3-(3-(((2-cyanophenoxy)(ethoxy)phosphoryl)methyl)-5-methylphenyl)acrylate

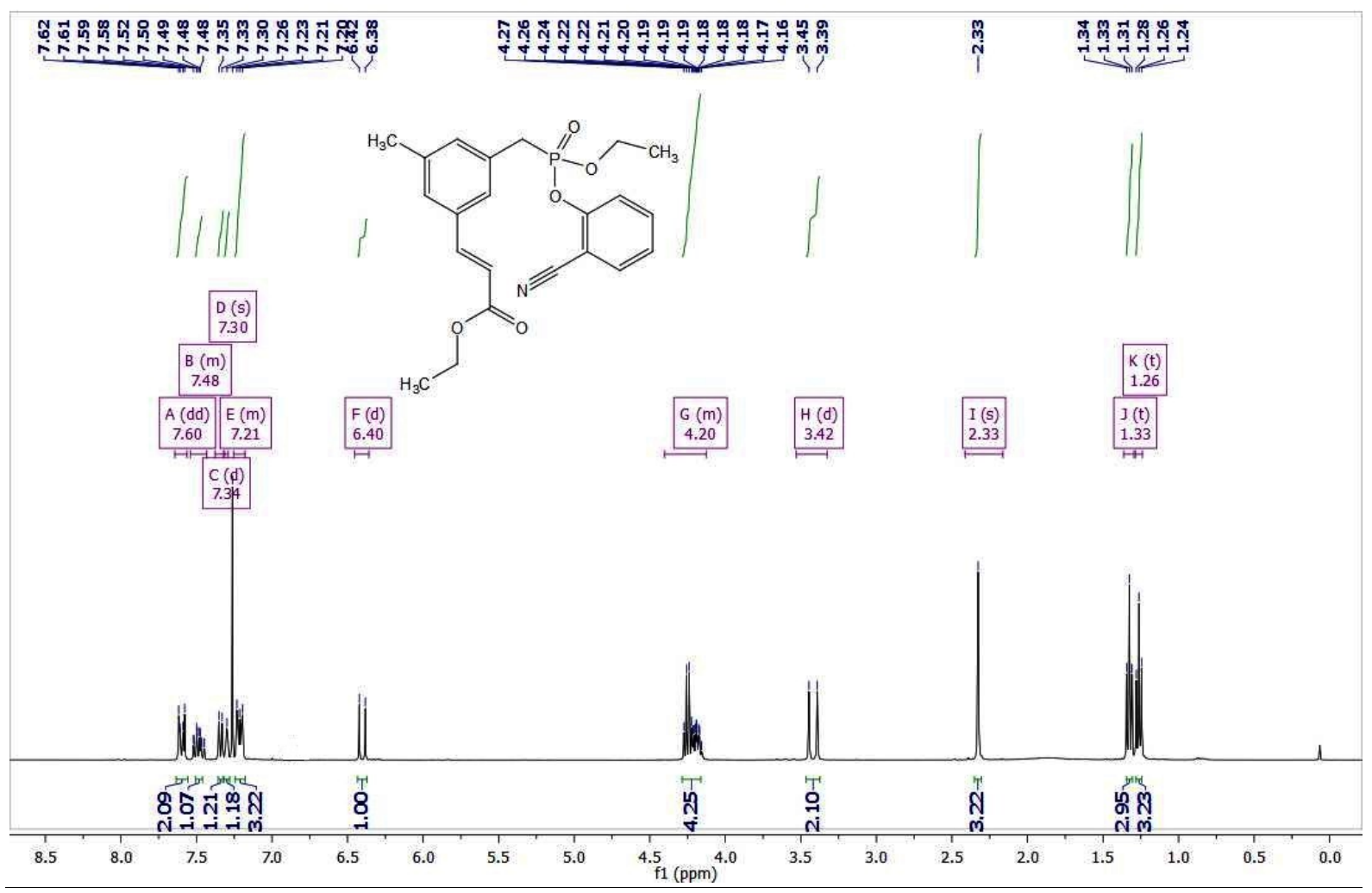

(E)-ethyl 3-(3-(((2-cyanophenoxy)(ethoxy)phosphoryl)methyl)-5-methylphenyl)acrylate

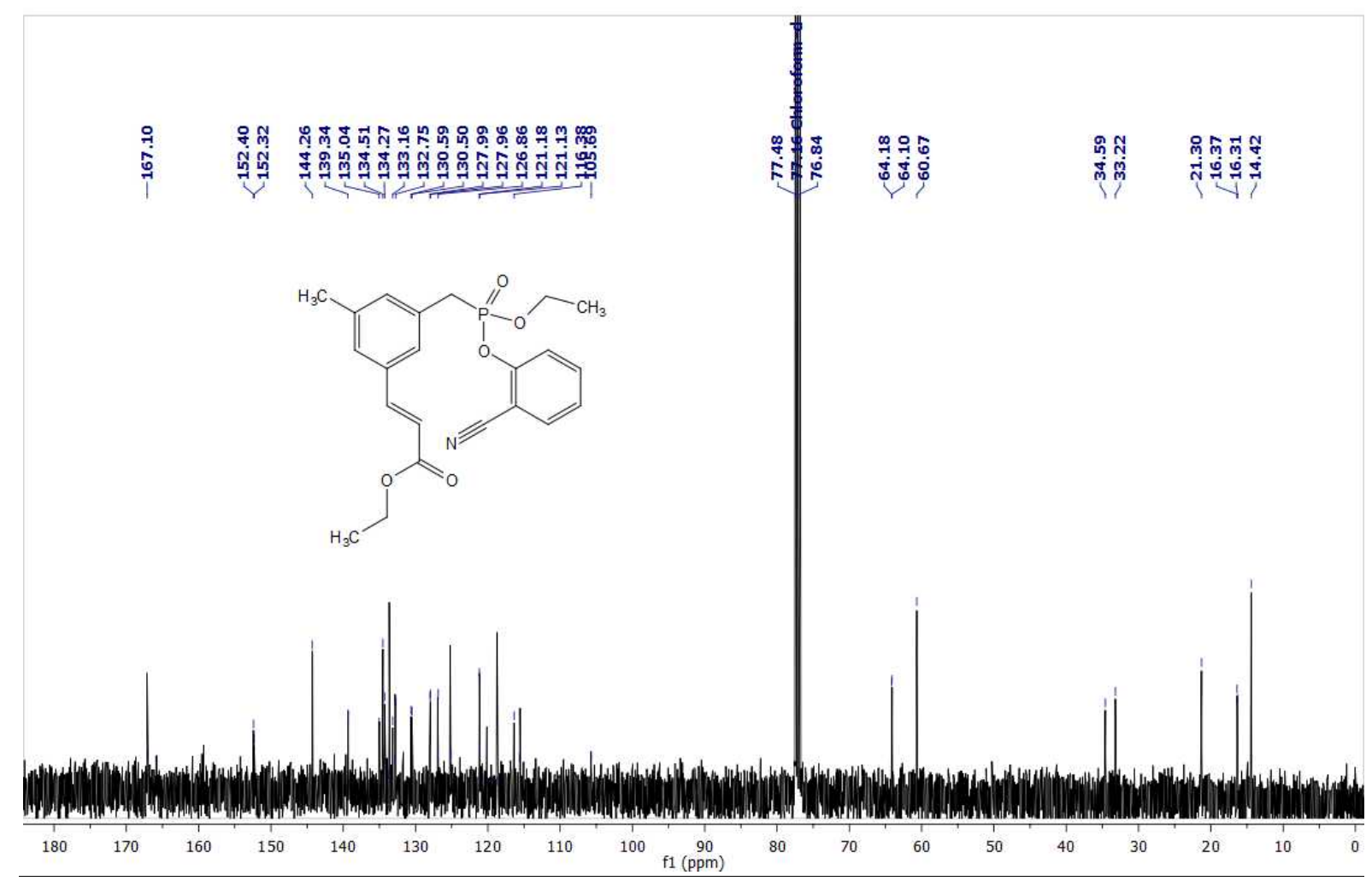


(E)-ethyl 3-(3-bromo-5-(((2-cyanophenoxy)(ethoxy)phosphoryl)methyl)-2-fluorophenyl)acrylate (2w):

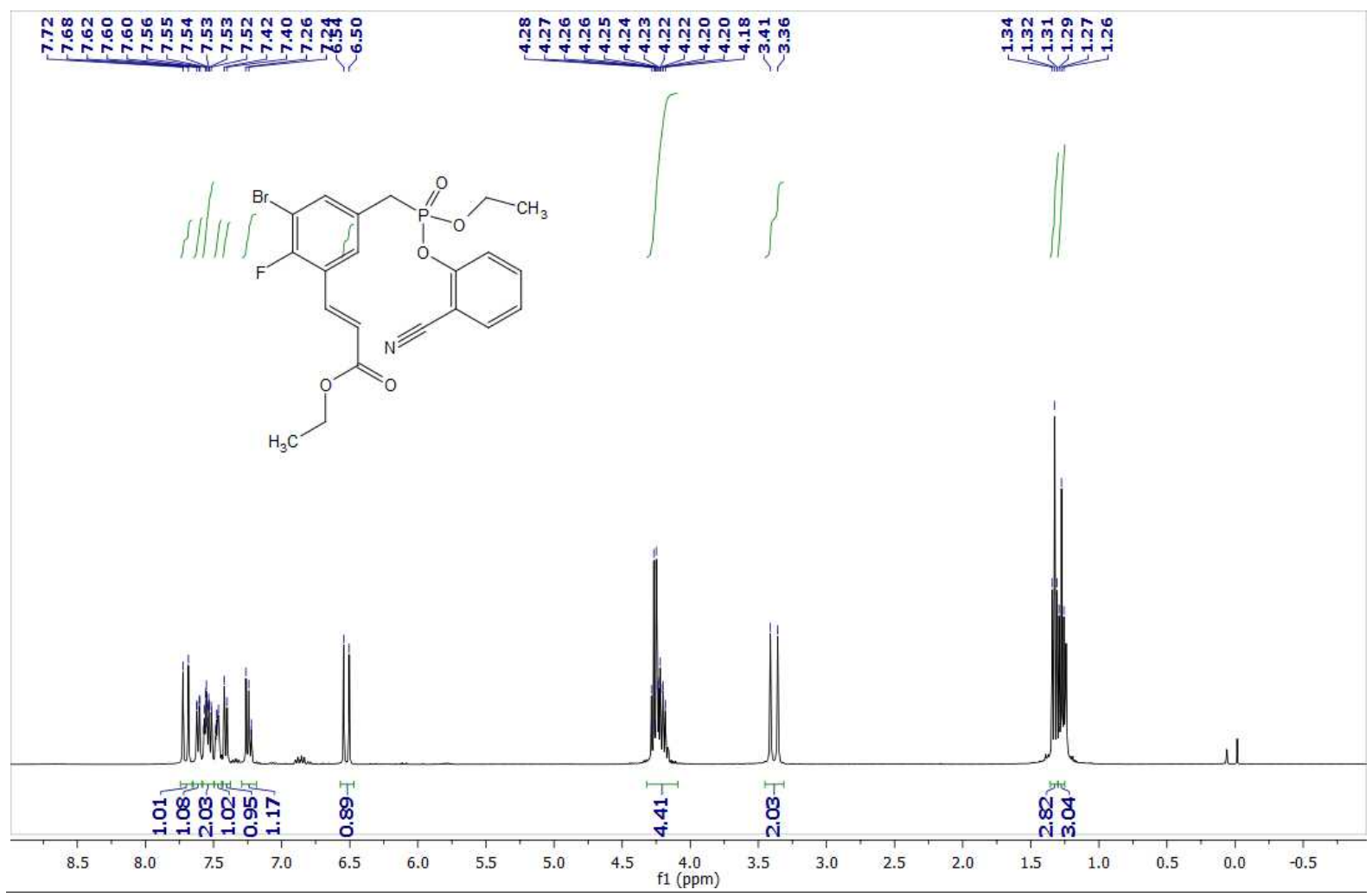

(E)-ethyl 3-(3-bromo-5-(((2-cyanophenoxy)(ethoxy)phosphoryl)methyl)-2-fluorophenyl)acrylate
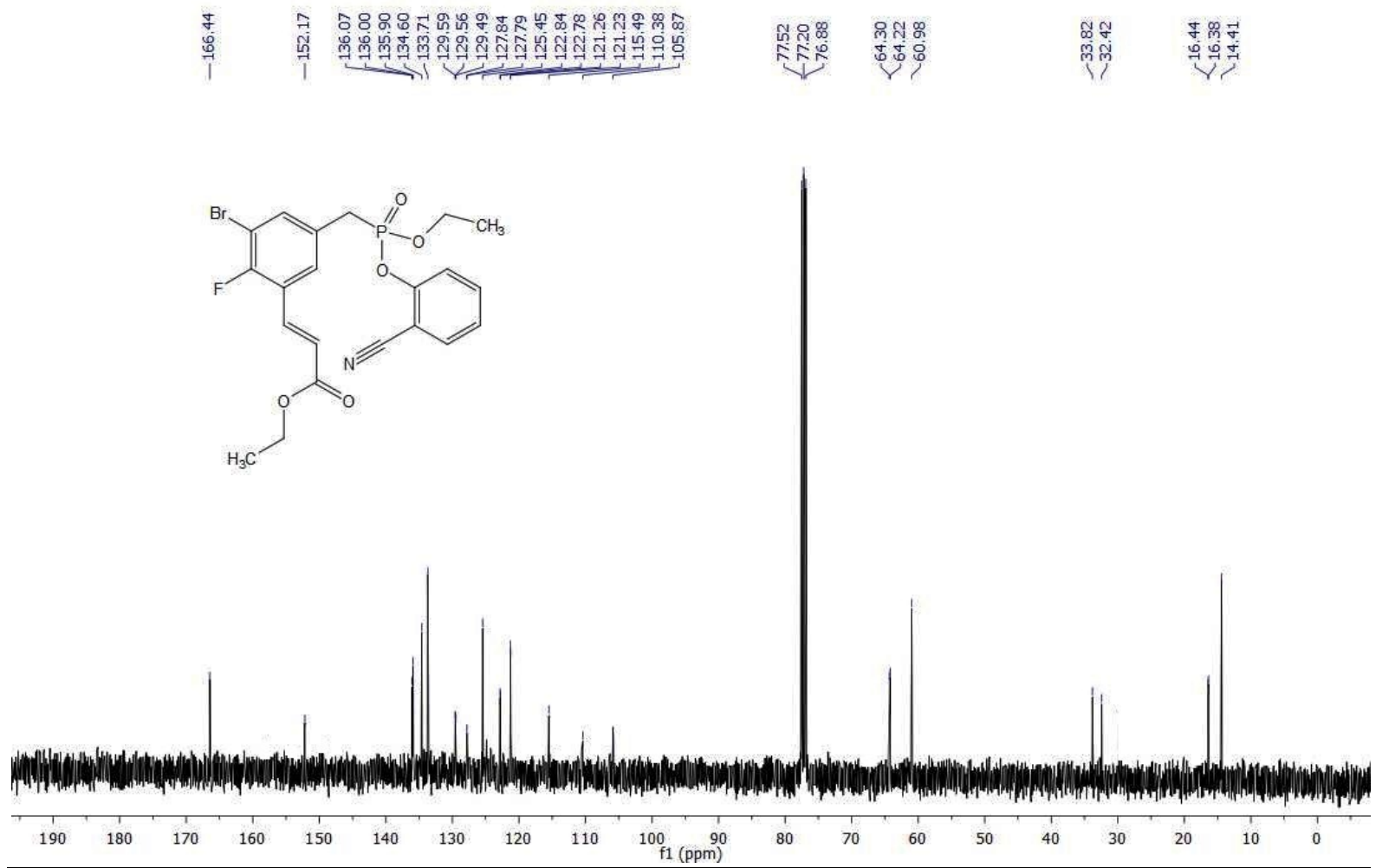
31P NMR:

$\underset{\substack{N \\ \text { N }}}{\stackrel{n}{N}}$

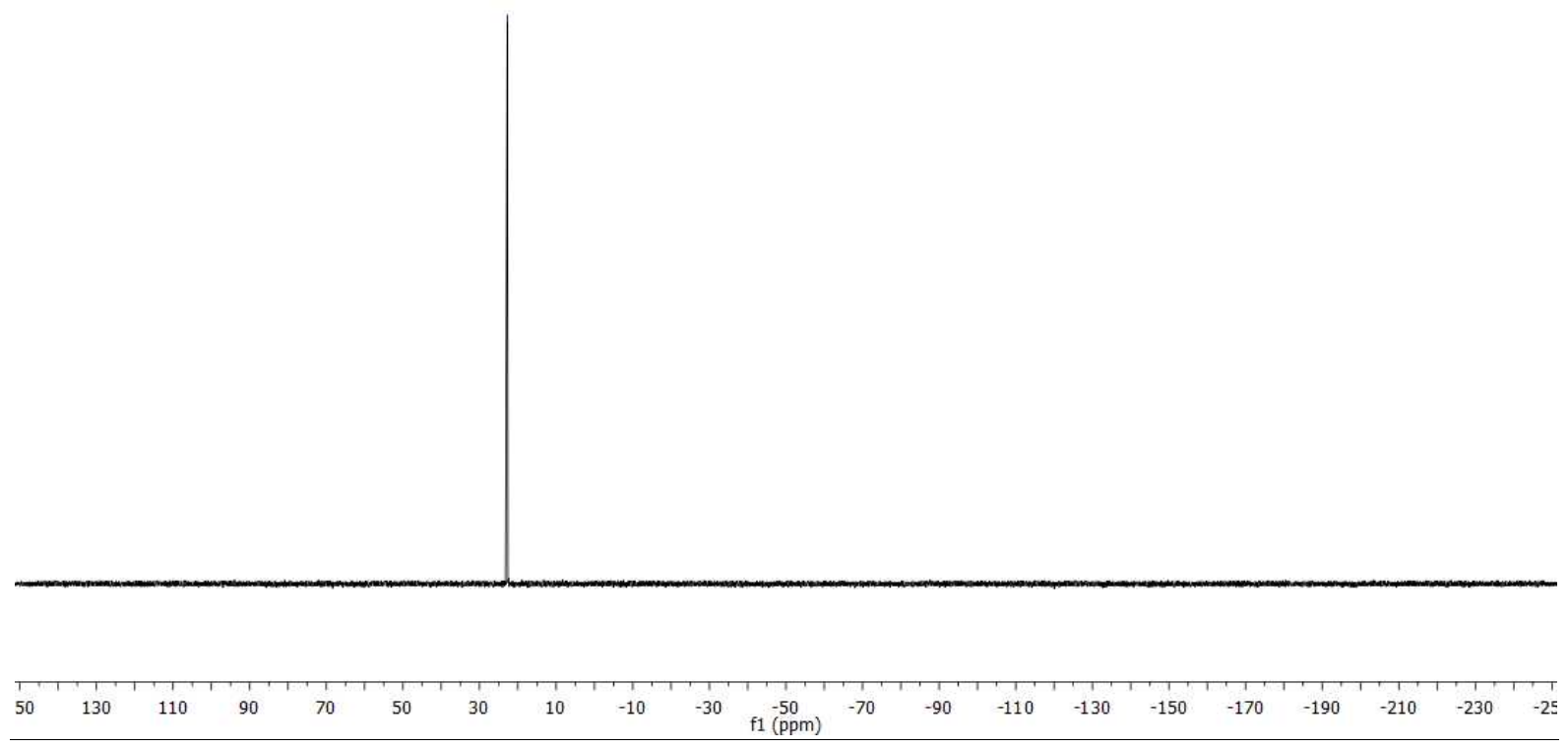




\section{Homo-diolefination and Hetero di-olefination:}

\section{(2E,2'E)-diethyl}

3,3'-(5-(((2-cyanophenoxy)(ethoxy)phosphoryl)methyl)-1,3phenylene)diacrylate (3a):

(2E,2'E)-diethyl 3,3'-(5-(((2-cyanophenoxy)(ethoxy)phosphoryl)methyl)-1,3-phenylene)diacrylate

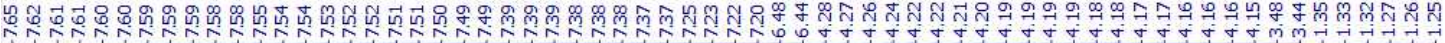

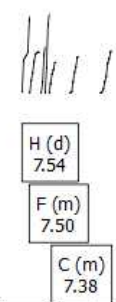

\begin{tabular}{|l|l|}
\hline$D(d)$ & $B(t)$ \\
7.63 & 7.22 \\
\hline
\end{tabular}

E(dt)

$E(d t)$
7.60

G(d)

7.53

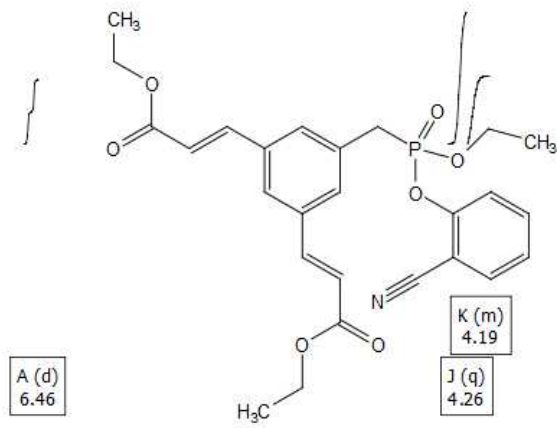<smiles>C1=CCCC1</smiles>
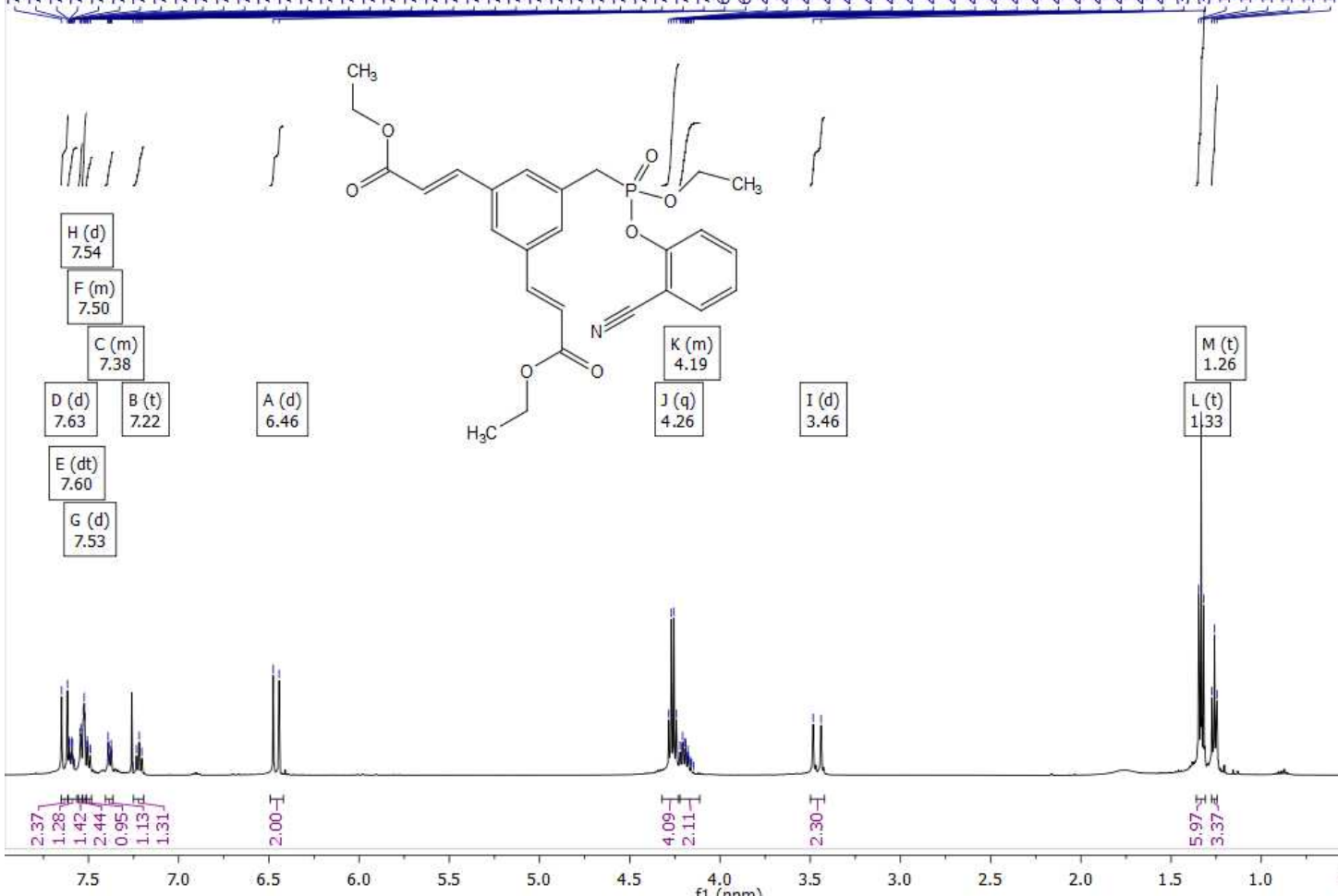

I (d)

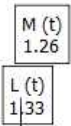

(2E,2'E)-diethyl 3,3'-(5-(((2-cyanophenoxy)(ethoxy)phosphoryl)methyl)-1,3-phenylene)diacrylate

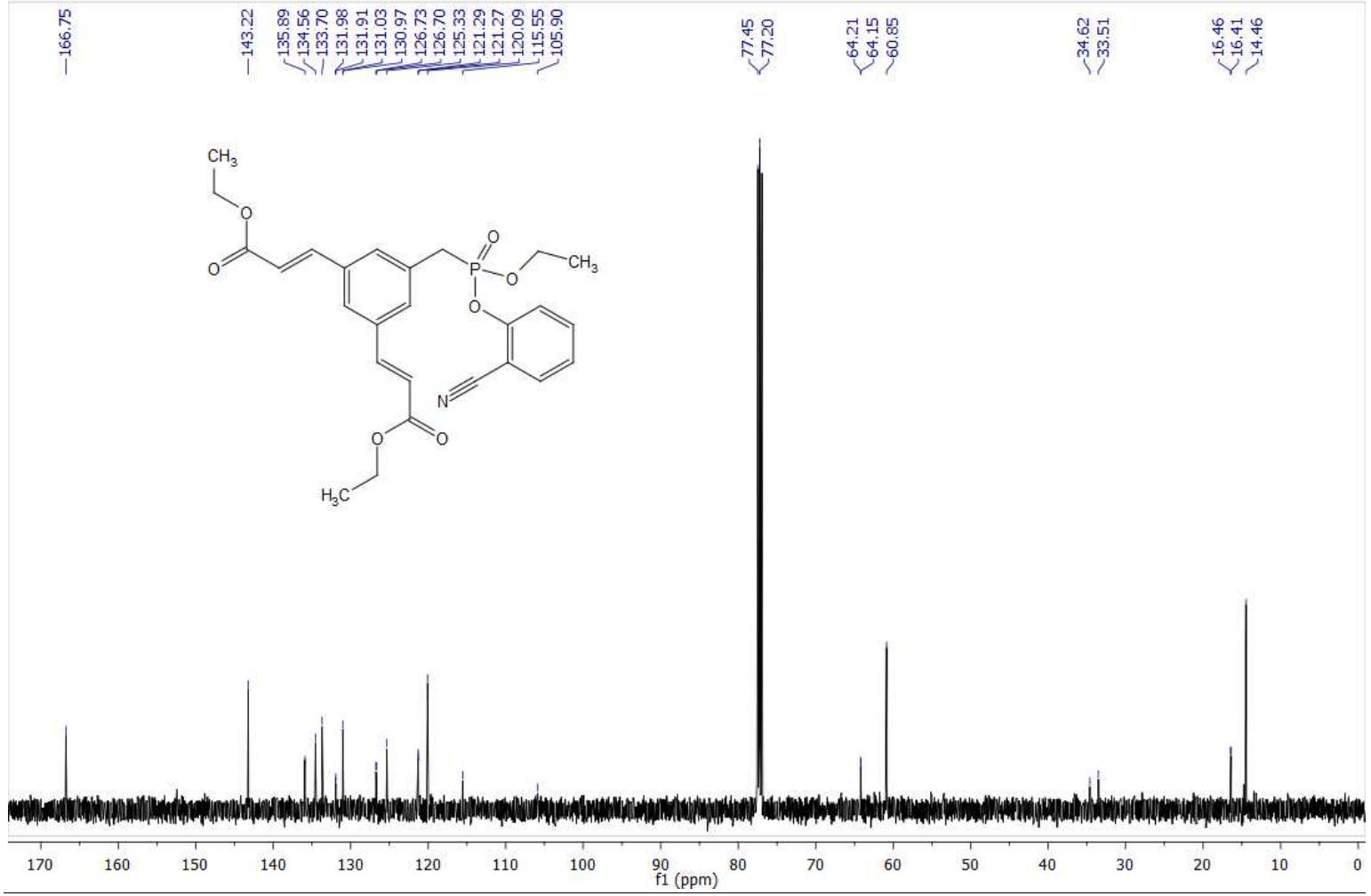




\section{2-cyanophenyl ethyl 3,5-bis((E)-3-oxobut-1-en-1-yl)benzylphosphonate (3b):}

2-cyanophenyl ethyl 3,5-bis((E)-3-oxobut-1-en-1-yl)benzylphosphonate

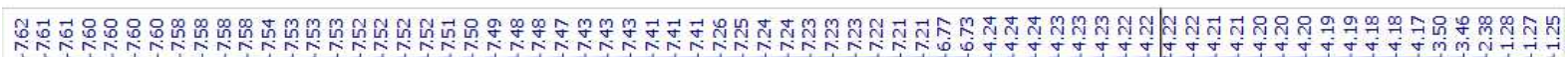<smiles>C#CC(C)C(=O)/C=C/c1cc(/C=C/C(=O)C(C)C)cc(CP(=O)(OCC)Oc2ccccc2C#N)c1</smiles>
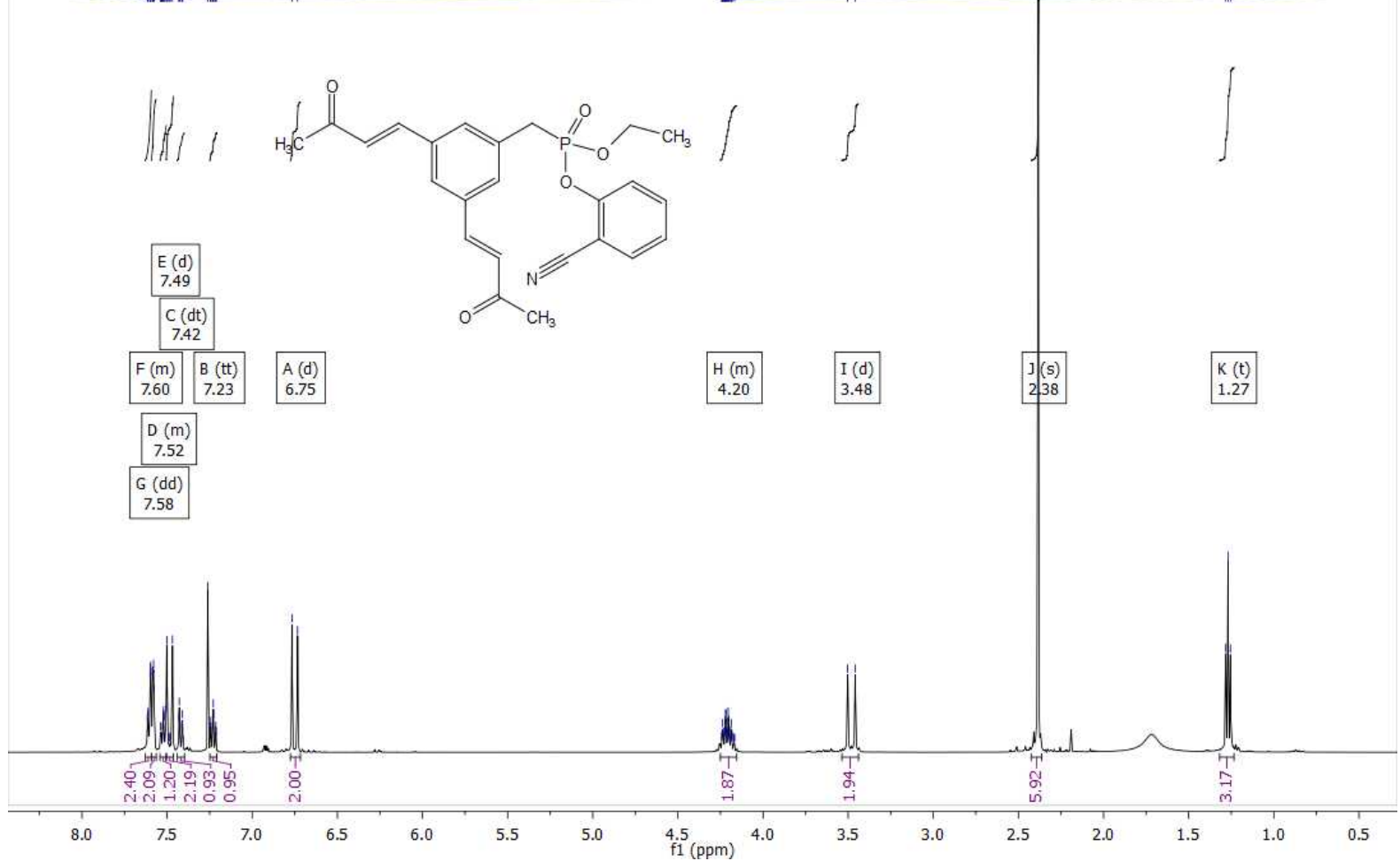

2-cyanophenyl ethyl 3,5-bis((E)-3-oxobut-1-en-1-yl)benzylphosphonate

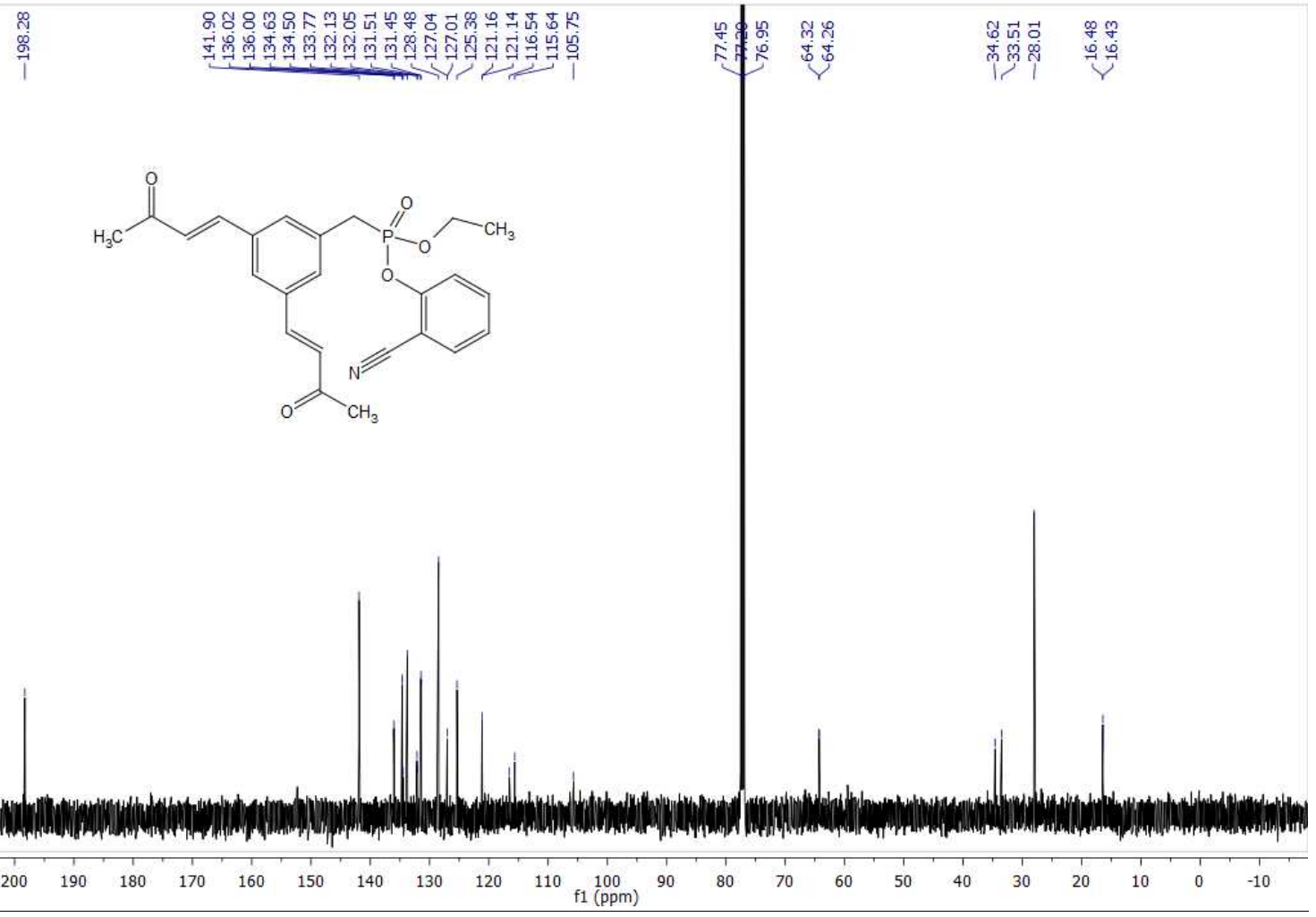


2-cyanophenyl ethyl ((6,6"-diacetyl-1,1",2,2",3,3",4,4"'-octahydro-[1,1':3',1"-terphenyl]-5'yl)methyl)phosphonate (3c):

2-cyanophenyl ethyl ((6,6"-diacetyl-1,1",2,2",3,3",4,4"-octahydro-[1,1':3',1"-terphenyl]-5'-yl)methyl)phosphonate

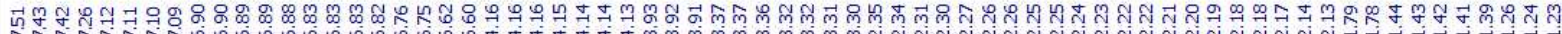

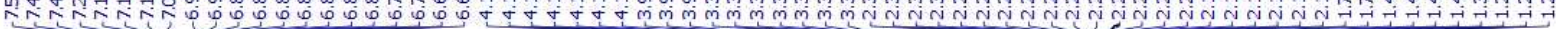

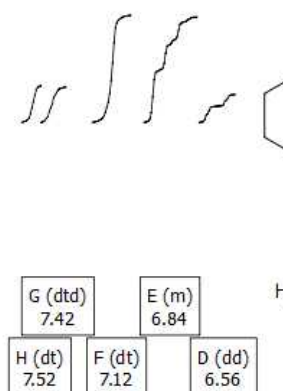<smiles>CC(=O)C1=CCCCC1c1cccc(C2CCCC=C2C(C)=O)c1</smiles><smiles>C=CC1C=CC=C1</smiles><smiles>C1CCC1</smiles><smiles>CC1C2CCC(C2)C1c1ccc(O)c(C#N)c1</smiles><smiles>C1CCCCC1</smiles>

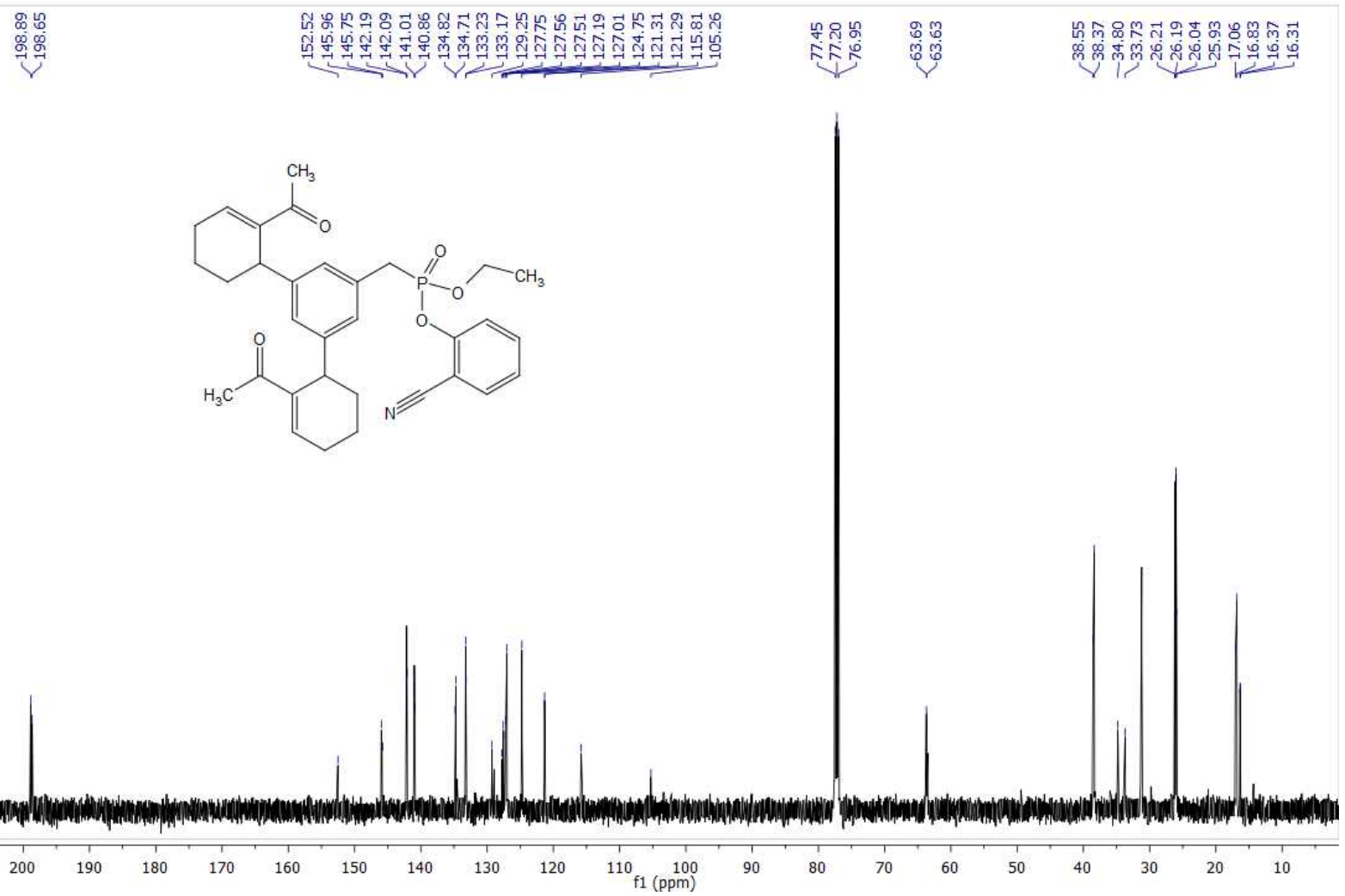


dimethyl 5,5'-(5-(((2-cyanophenoxy)(ethoxy)phosphoryl)methyl)-1,3-phenylene)bis(cyclopent-1enecarboxylate) (3d):

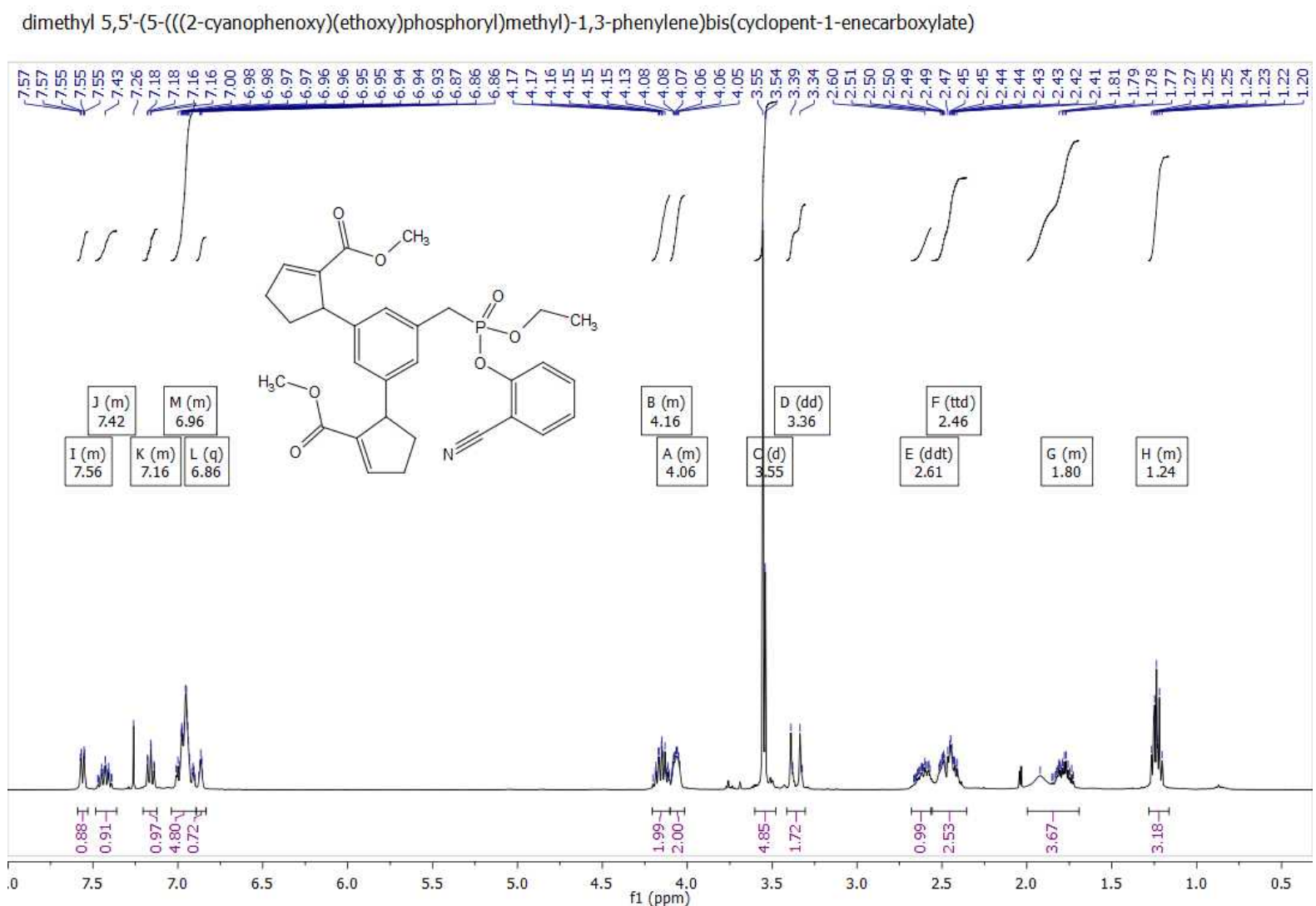

dimethyl 5,5'-(5-(((2-cyanophenoxy)(ethoxy)phosphoryl)methyl)-1,3-phenylene)bis(cyclopent-1-enecarboxylate)

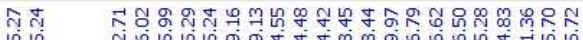

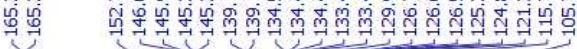

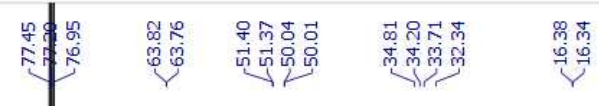<smiles>CCOP(=O)(Cc1cc(C2CCC=C2C(=O)OC)cc(C2CCC=C2C(=O)OC)c1)Oc1ccccc1C#N</smiles>

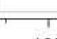

$80 \quad 170$

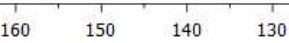

120

${ }^{100}{ }_{\mathrm{f} 1(\mathrm{ppm})}^{90}$

80

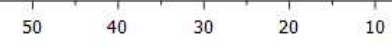


(E)-ethyl yl)phenyl)acrylate (3e):

(E)-ethyl 3-(3-(((2-cyanophenoxy)(ethoxy)phosphoryl)methyl)-5-((E)-3-oxobut-1-en-1-yl)phenyl)acrylate

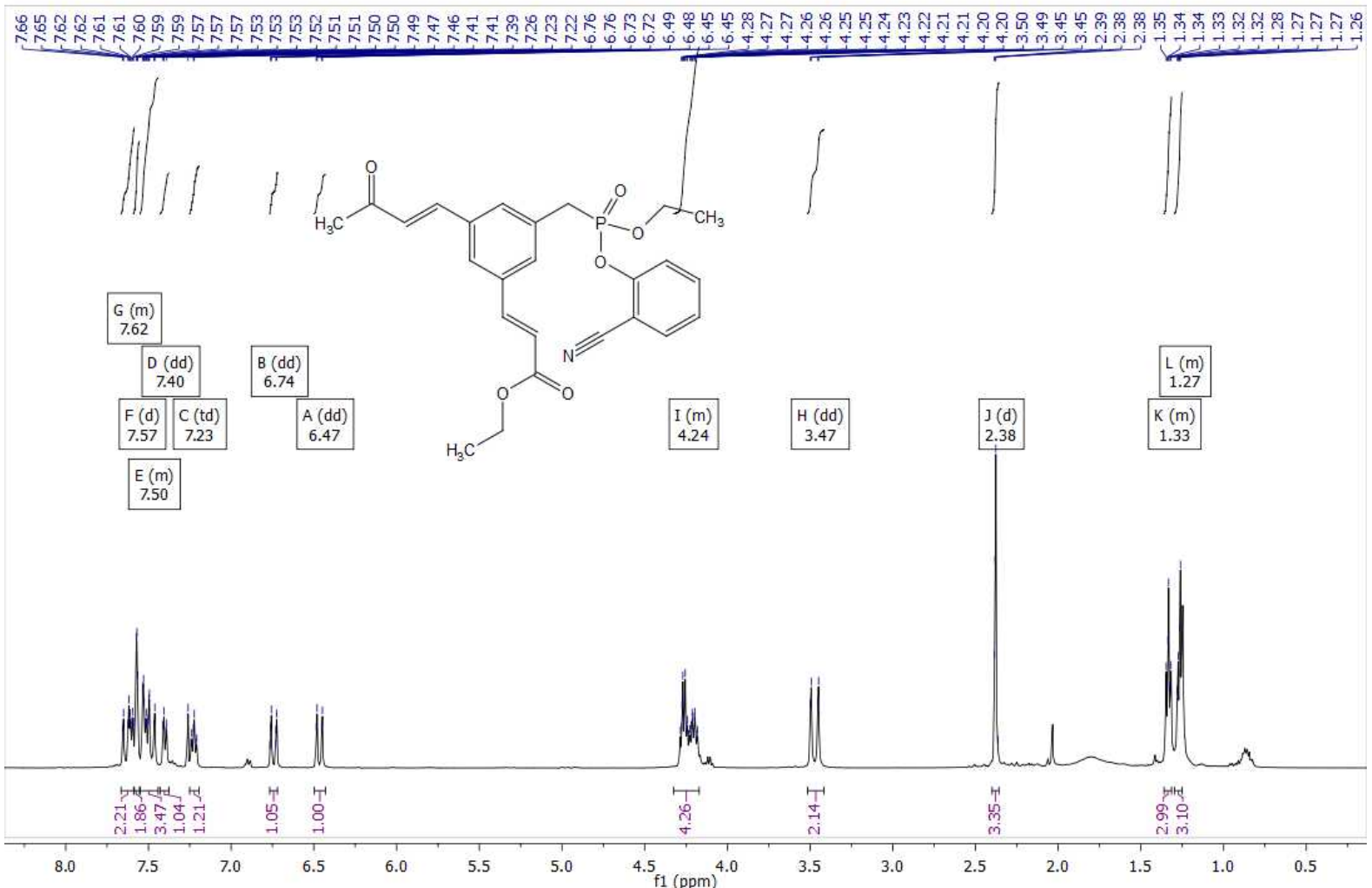

(E)-ethyl 3-(3-(((2-cyanophenoxy)(ethoxy)phosphoryl)methyl)-5-((E)-3-oxobut-1-en-1-yl)phenyl)acrylate

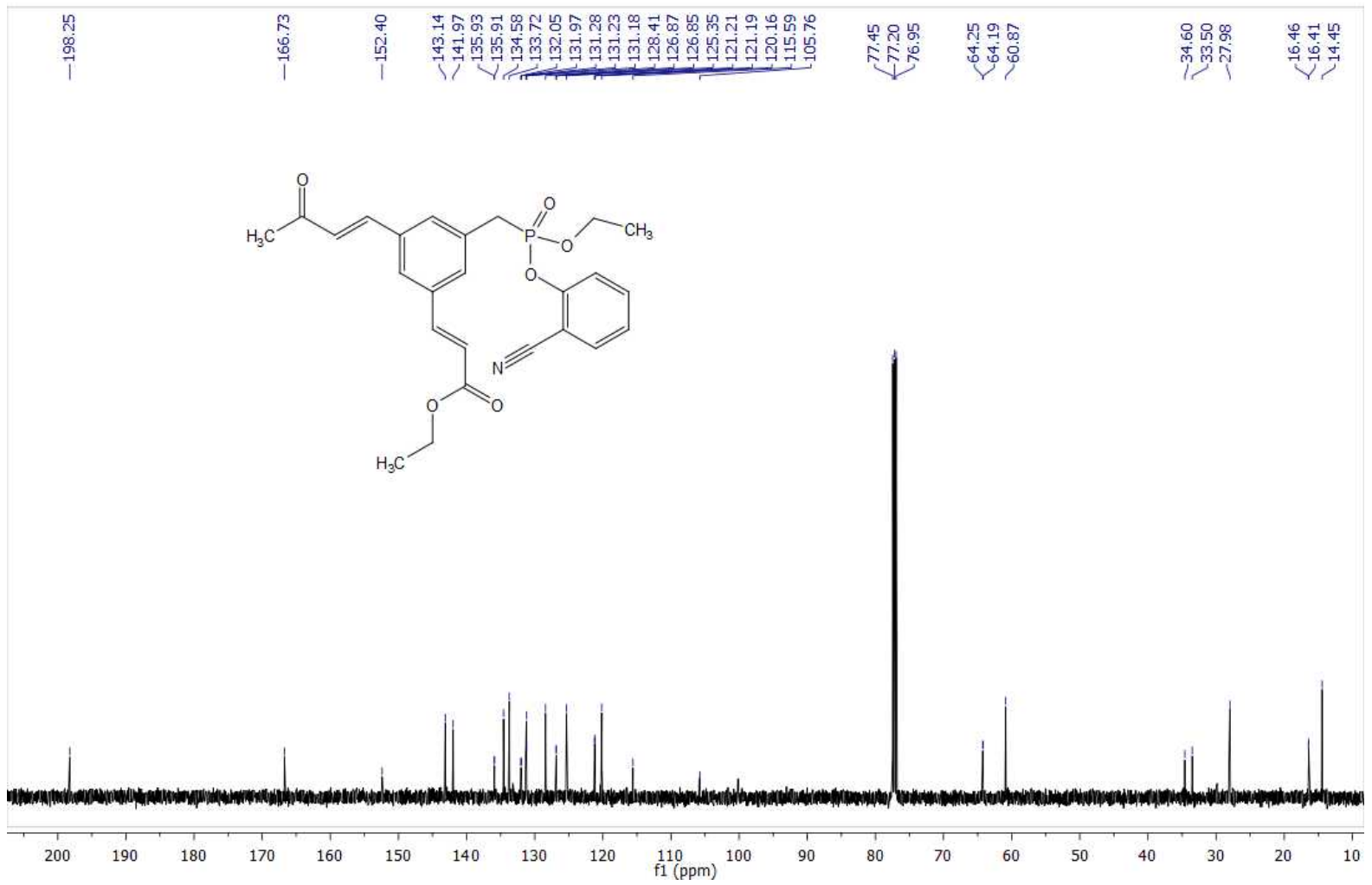


(E)-ethyl 3-(3-(((2-cyanophenoxy)(ethoxy)phosphoryl)methyl)-5-((E)-3-methoxy-3oxoprop-1-en-1-yl)phenyl)acrylate (3f):

(E)-ethyl 3-(3-(((2-cyanophenoxy)(ethoxy)phosphoryl)methyl)-5-((E)-3-methoxy-3-oxoprop-1-en-1-yl)phenyl)acrylate

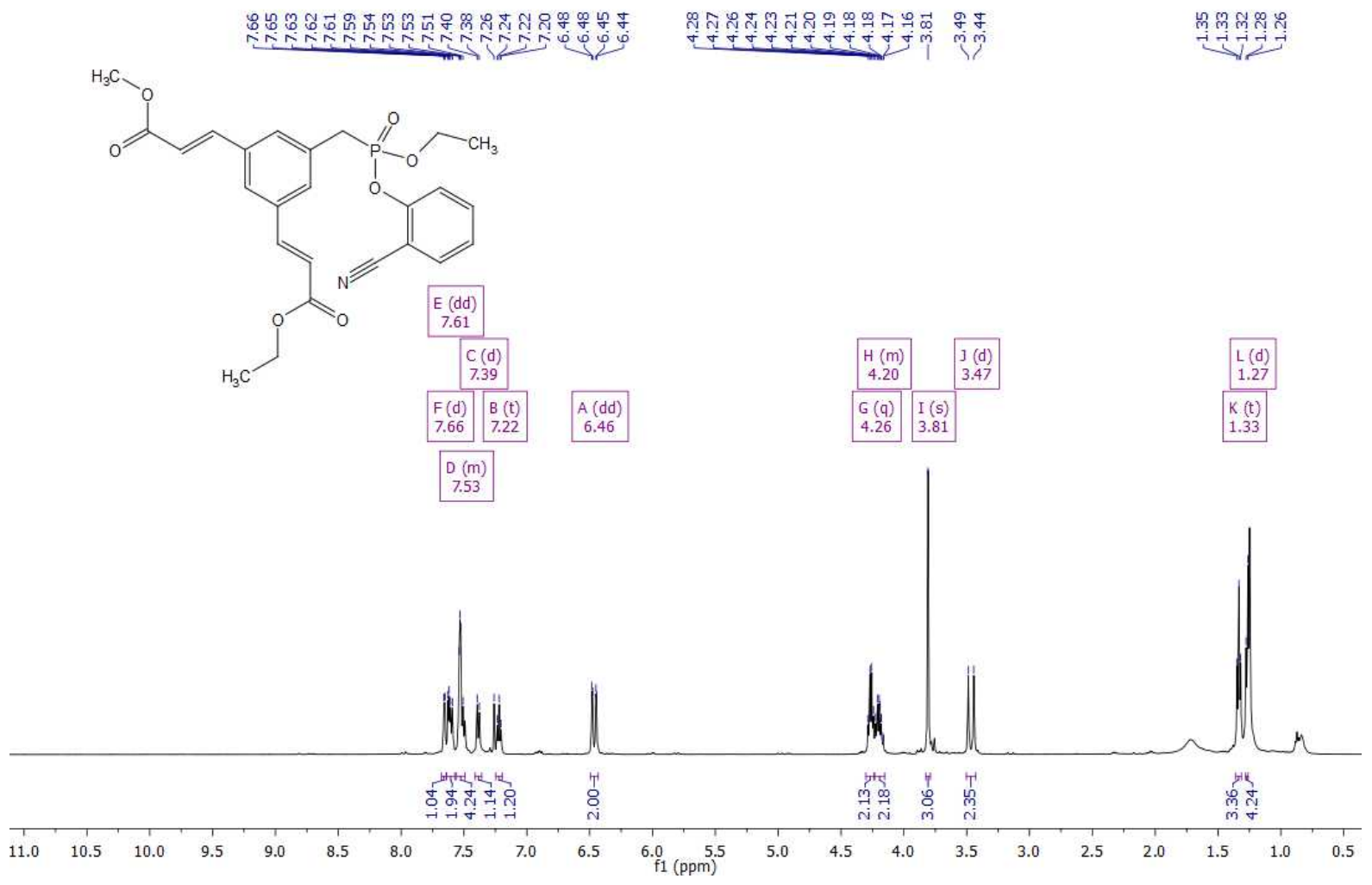

(E)-ethyl 3-(3-(((2-cyanophenoxy)(ethoxy)phosphoryl)methyl)-5-((E)-3-methoxy-3-oxoprop-1-en-1-yl)phenyl)acrylate

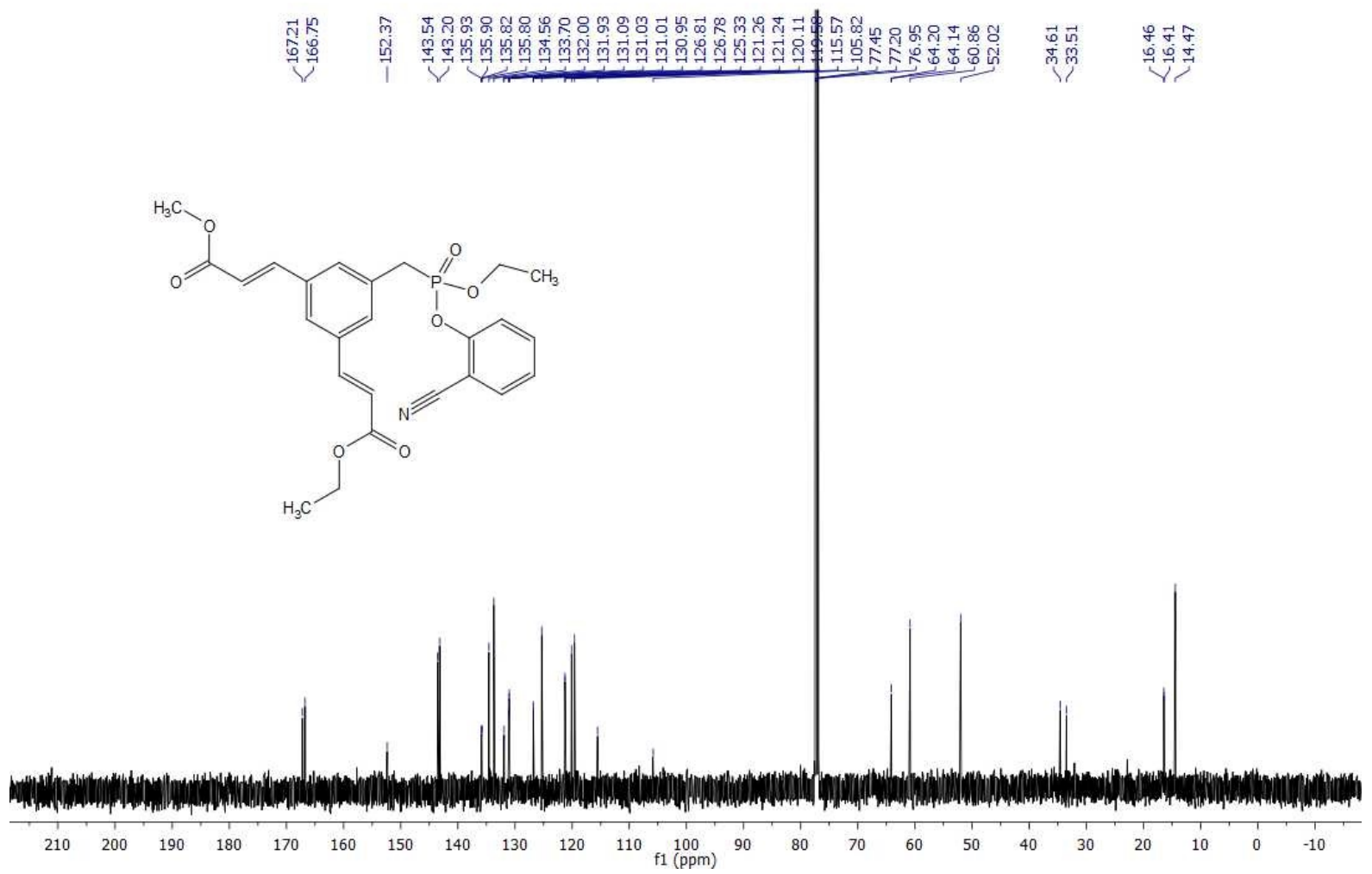


(E)-methyl 3-(3-(((2-cyanophenoxy)(ethoxy)phosphoryl)methyl)-5-((E)-3-oxobut-1-en-1yl)phenyl)acrylate (3g):

(E)-methyl 3-(3-(((2-cyanophenoxy)(ethoxy)phosphoryl)methyl)-5-((E)-3-oxobut-1-en-1-yl)phenyl)acrylate

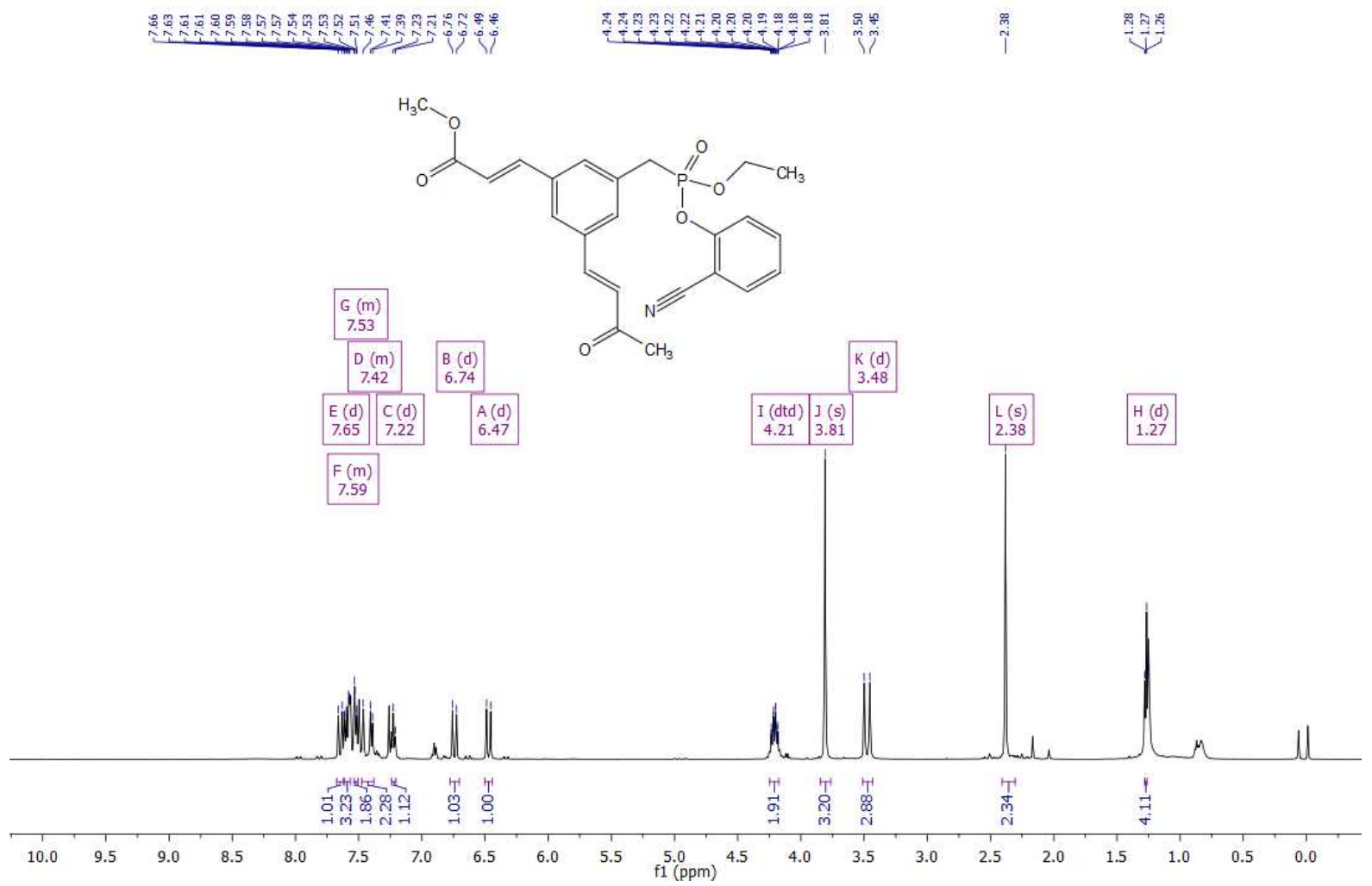

(E)-methyl 3-(3-(((2-cyanophenoxy)(ethoxy)phosphoryl)methyl)-5-((E)-3-oxobut-1-en-1-yl)phenyl)acrylate

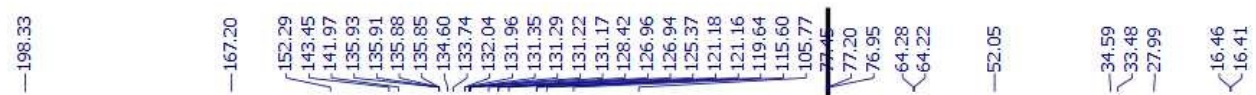<smiles>CCOP(=O)(Cc1cc(/C=C/C(C)=O)cc(/C=C/C(=O)OC)c1)Oc1ccccc1C#N</smiles>

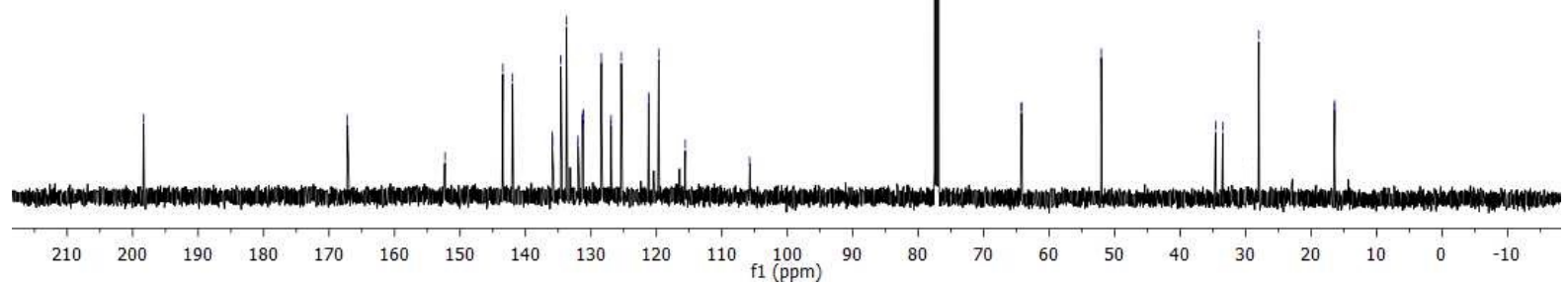


31P NMR:

ขึ

$\stackrel{\mathscr{N}}{i}$

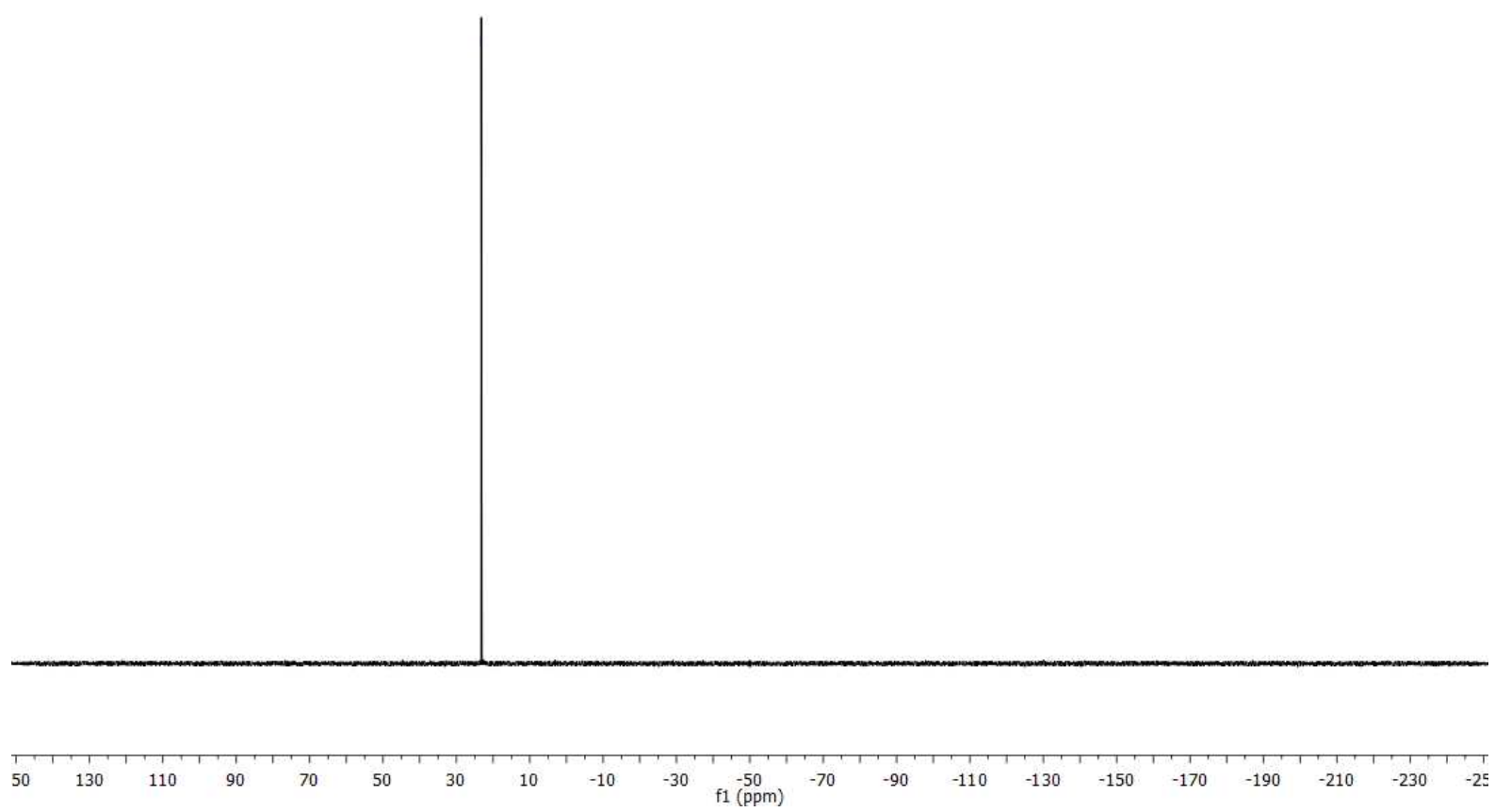


(E)-methyl

3-(3-(((2-cyanophenoxy)(ethoxy)phosphoryl)methyl)-5-((E)-2(methylsulfonyl)vinyl)phenyl)acrylate (3h):

(E)-methyl 3-(3-(((2-cyanophenoxy)(ethoxy)phosphoryl)methyl)-5-((E)-2-(methylsulfonyl)vinyl)phenyl)acrylate

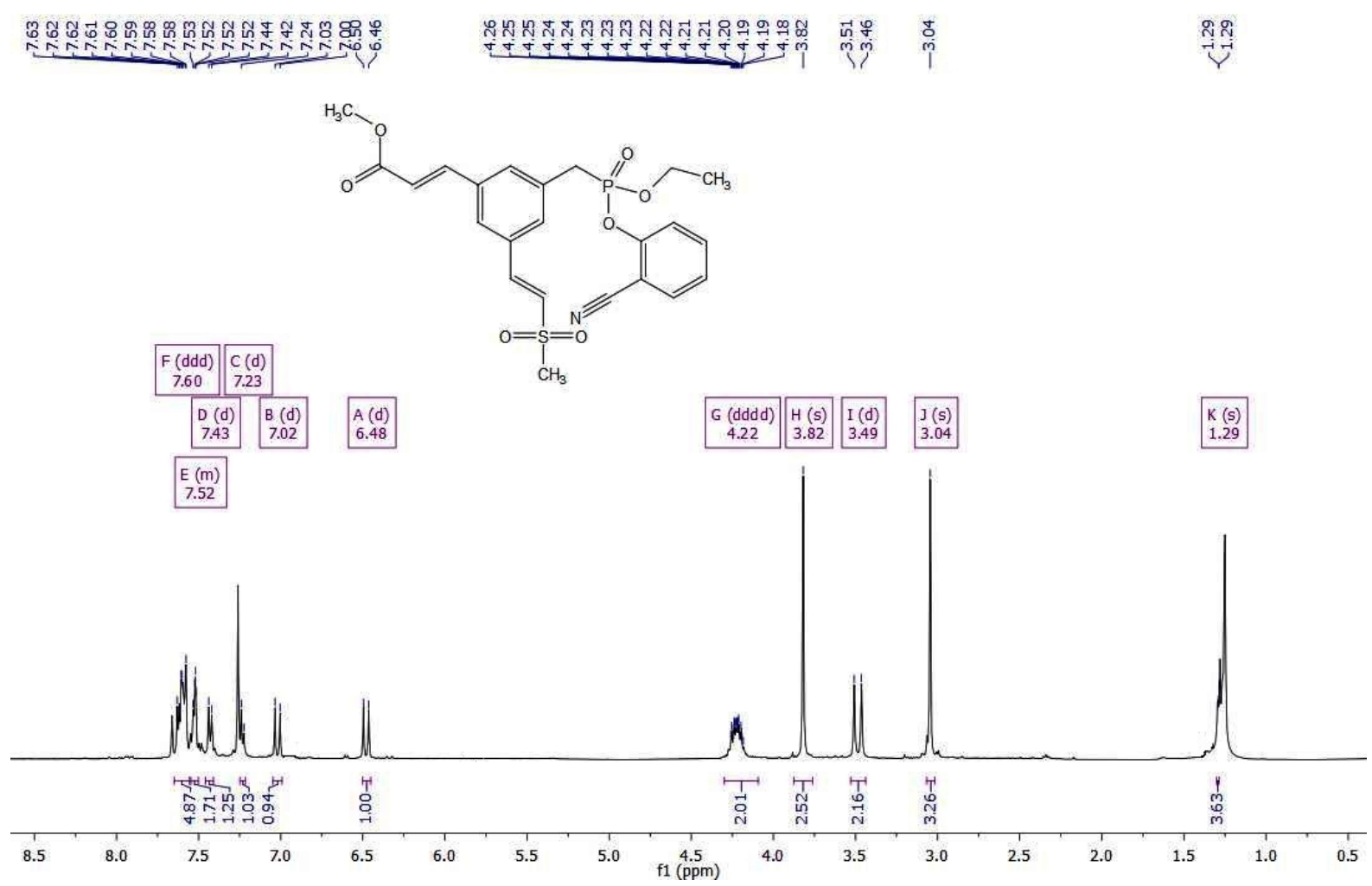

(E)-methyl 3-(3-(((2-cyanophenoxy)(ethoxy)phosphoryl)methyl)-5-((E)-2-(methylsulfonyl)vinyl)phenyl)acrylate

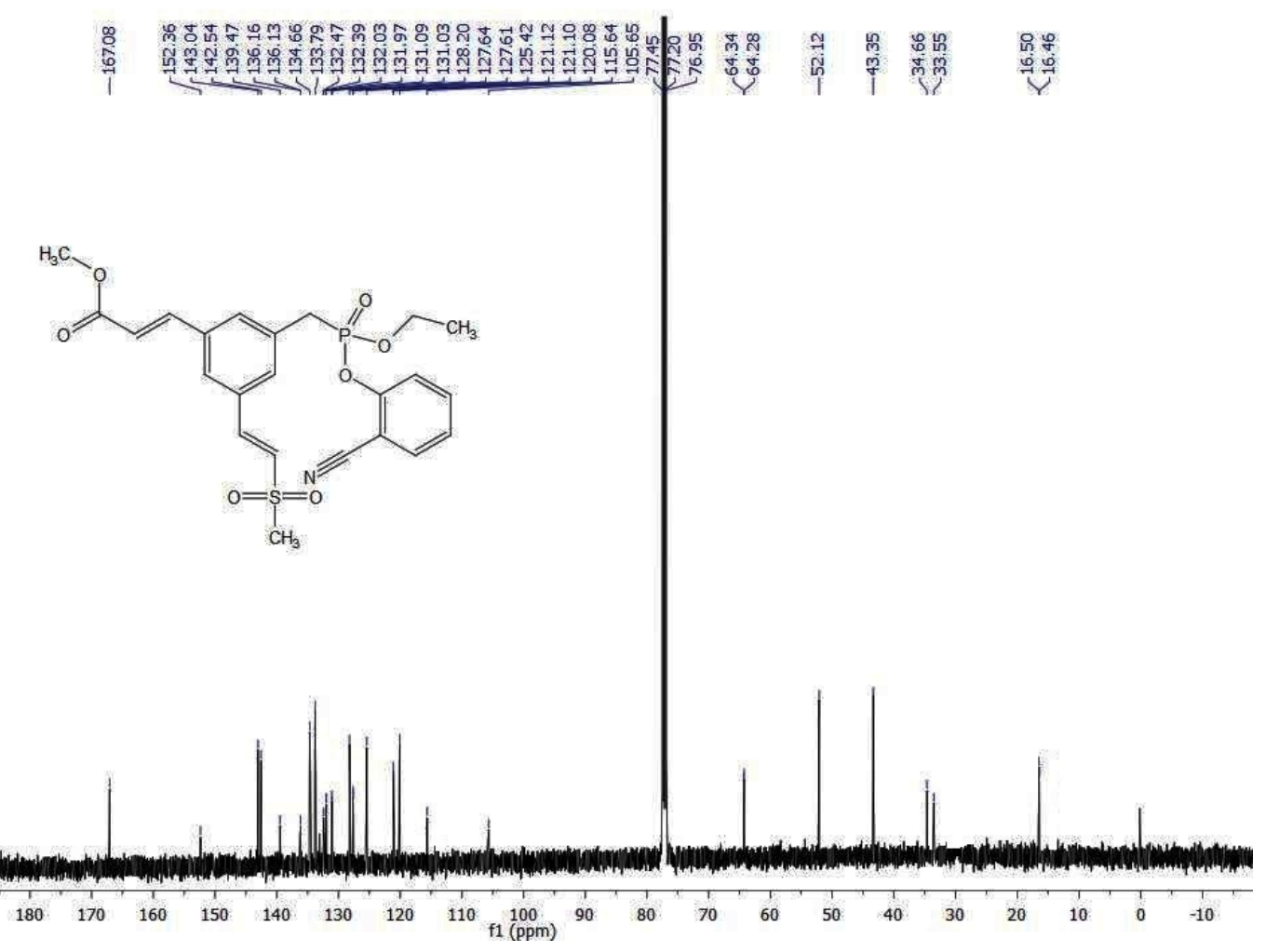


31P NMR:

$\overrightarrow{\mathrm{N}}$

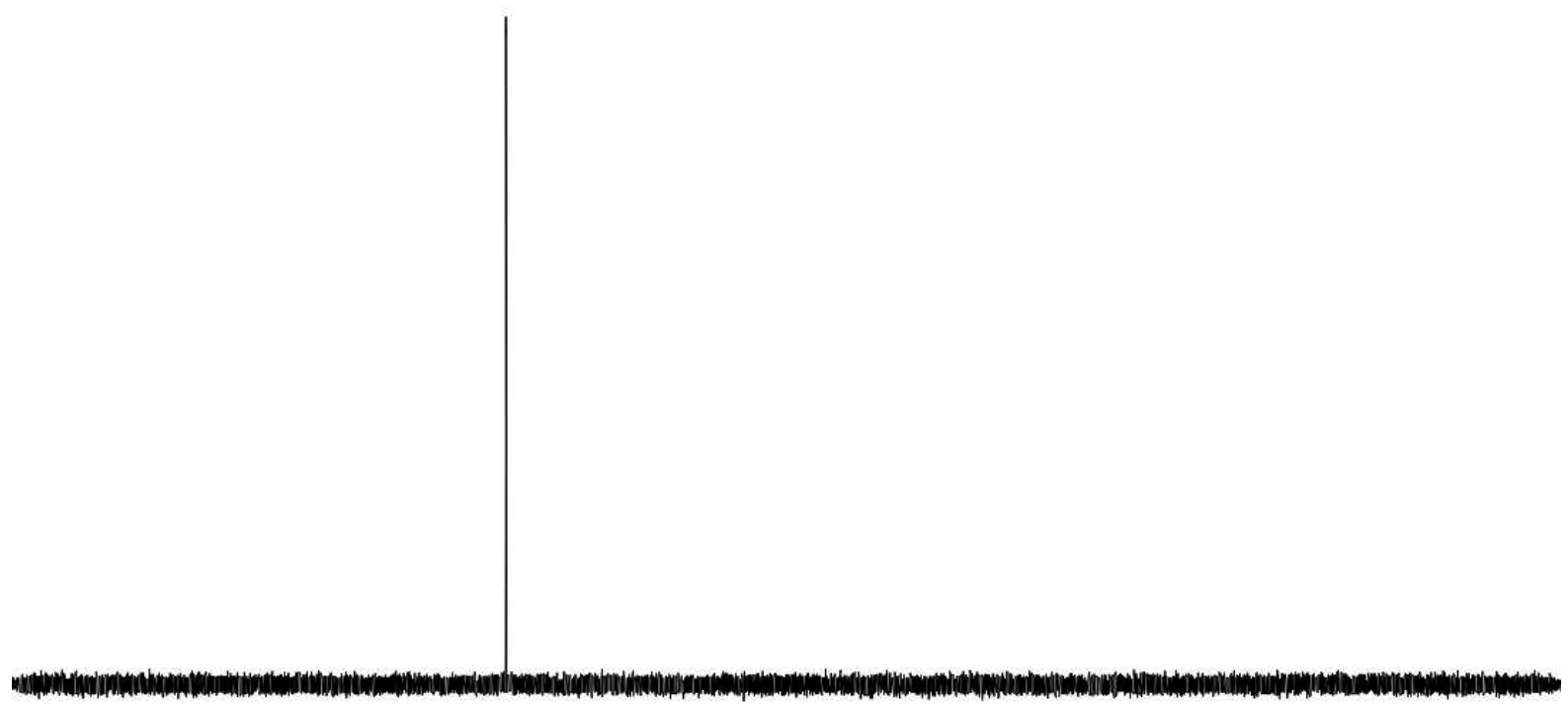

$50 \begin{array}{lllllllllllllllllllllllll} & 130 & 110 & 90 & 70 & 50 & 30 & 10 & -10 & -30 & -50 & -70 & -90 & -110 & -130 & -150 & -170 & -190 & -210 & -230 & -25\end{array}$ 


\section{Meta-Hydroxylation:}

2-cyanophenyl ethyl 3-hydroxybenzylphosphonate (4a):

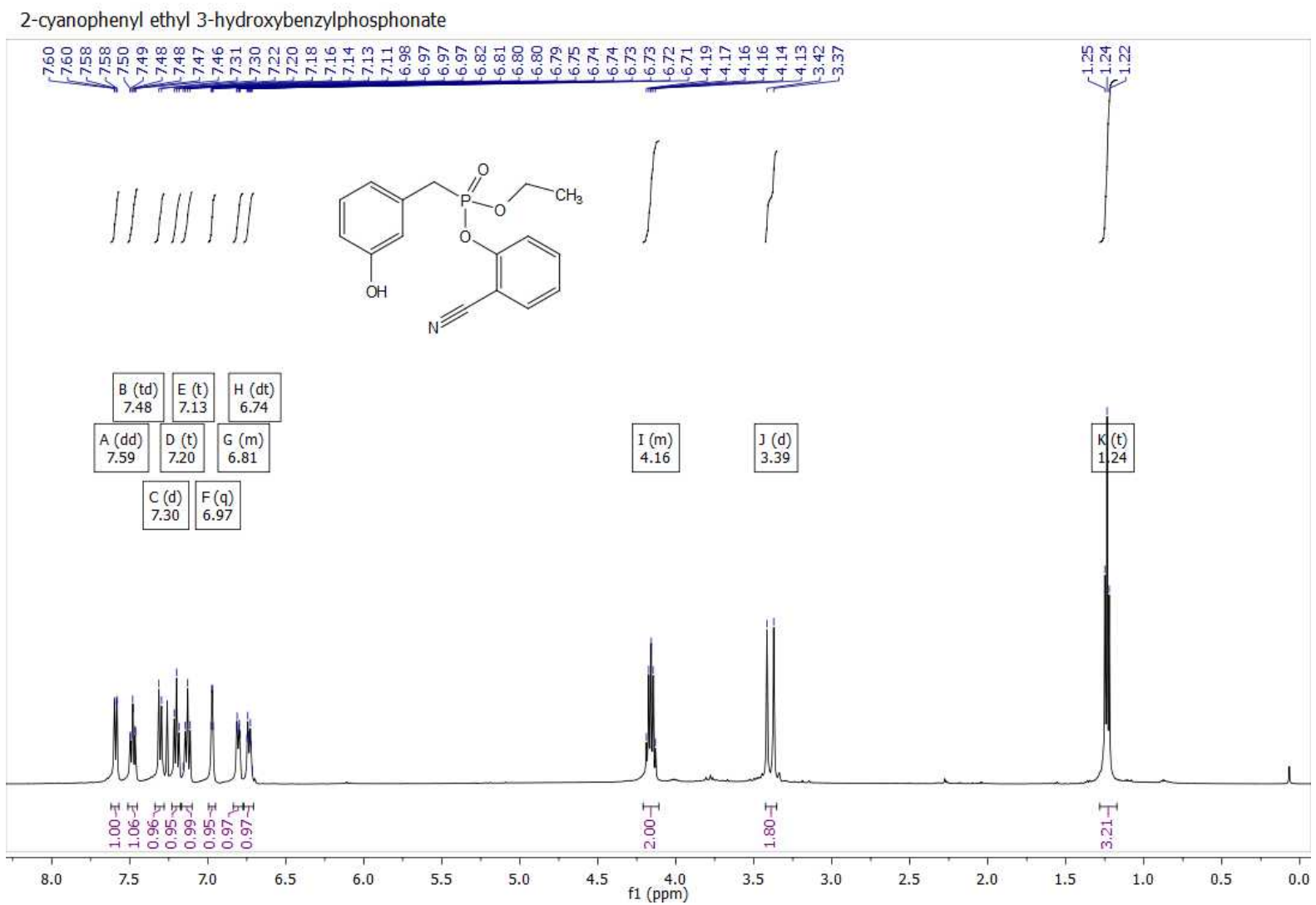

2-cyanophenyl ethyl 3-hydroxybenzylphosphonate

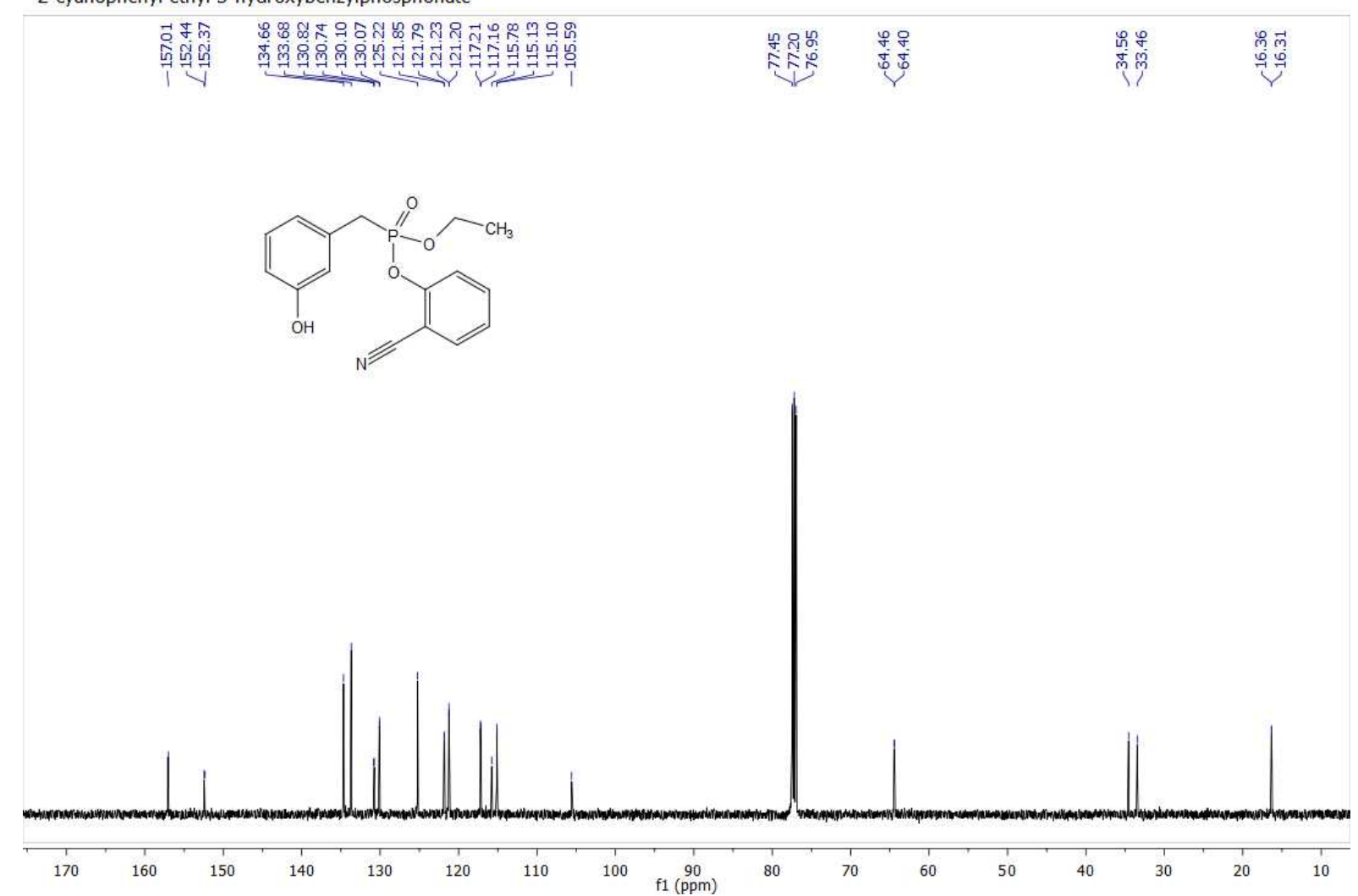


31P NMR:

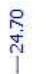

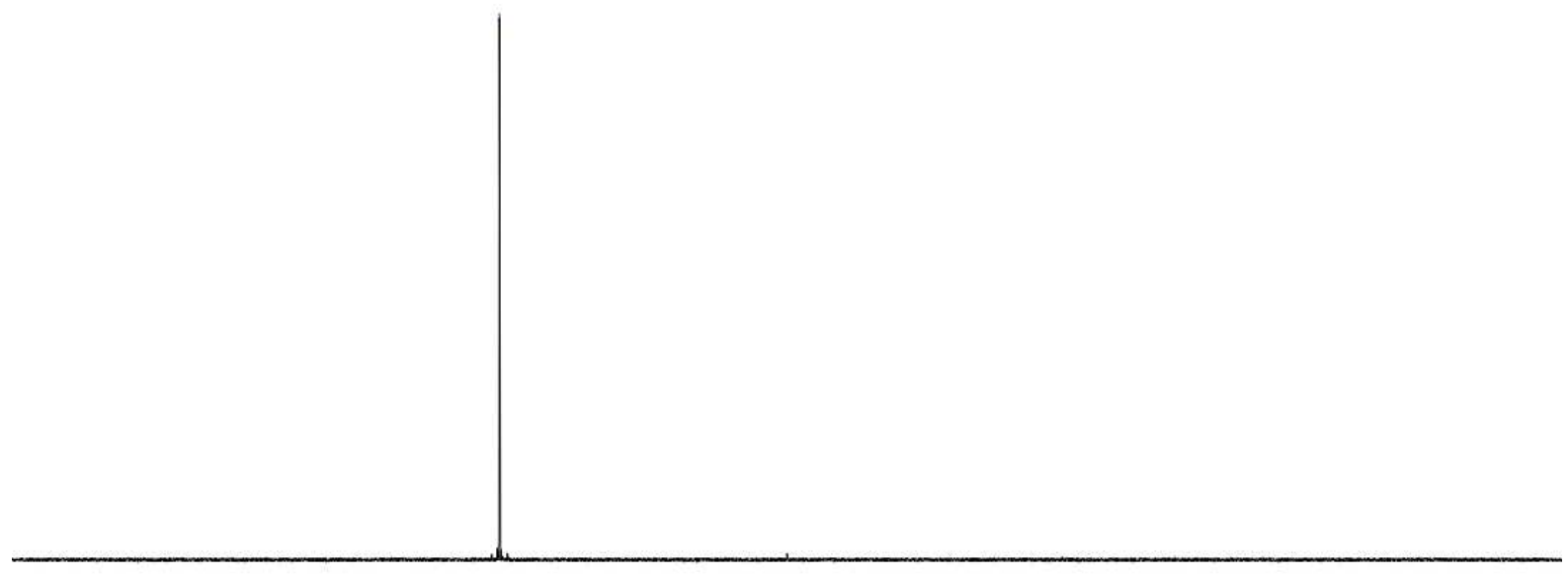

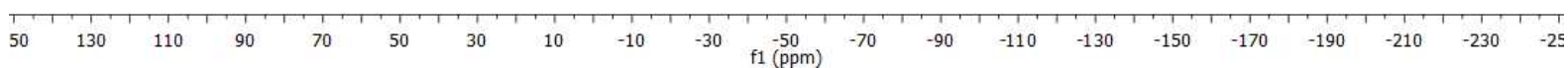




\section{2-cyanophenyl ethyl 3-hydroxy-4-methylbenzylphosphonate (4b):}

2-cyanophenyl ethyl 3-hydroxy-4-methylbenzylphosphonate

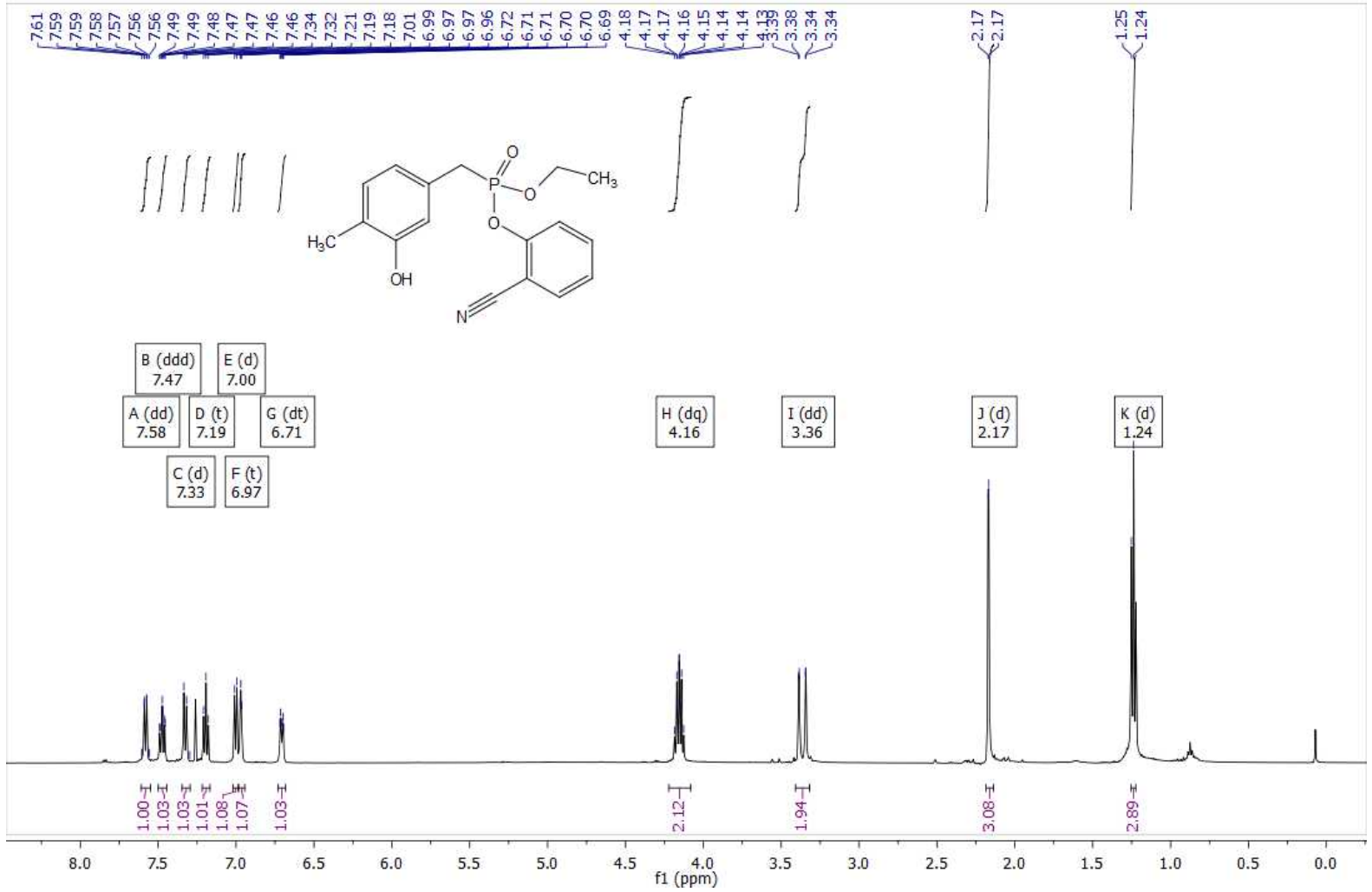

2-cyanophenyl ethyl 3-hydroxy-4-methylbenzylphosphonate

\begin{tabular}{|c|c|c|}
\hline 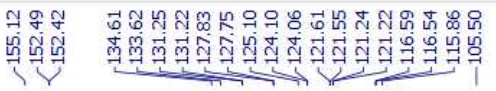 & 号昌 & 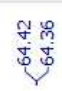 \\
\hline
\end{tabular}<smiles>CCOP(=O)(Cc1ccc(C)c(O)c1)Oc1ccccc1C#N</smiles>

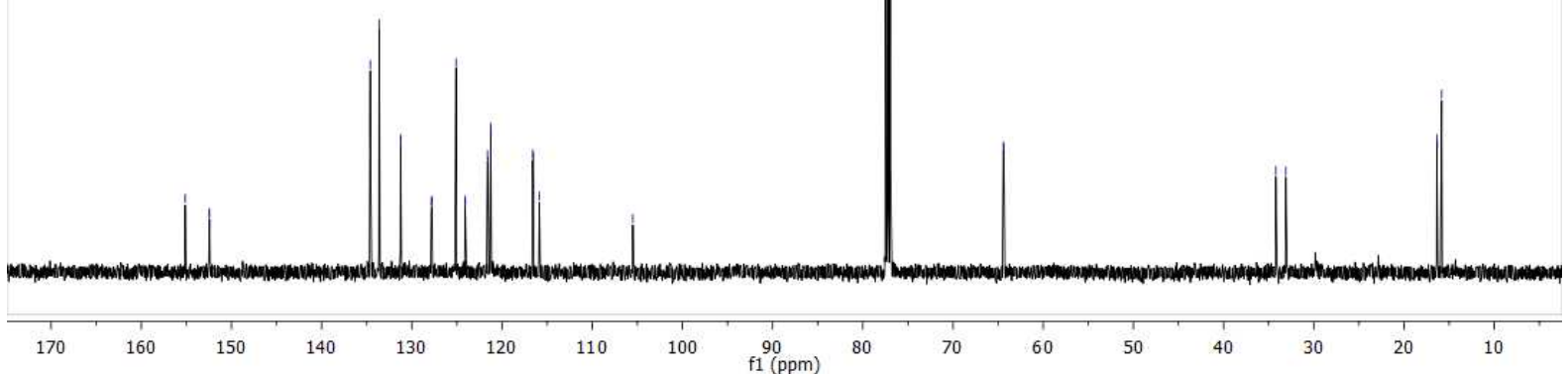


2-cyanophenyl ethyl 4-chloro-3-hydroxybenzylphosphonate (4c):

2-cyanophenyl ethyl 4-chloro-3-hydroxybenzylphosphonate

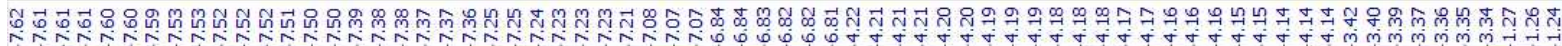<smiles>C=CC=CC</smiles><smiles>CCOP(=O)(Cc1ccc(Cl)c(O)c1)Oc1ccccc1C#N</smiles>

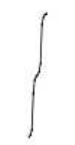

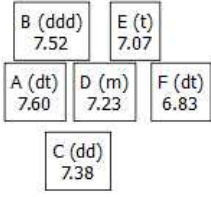
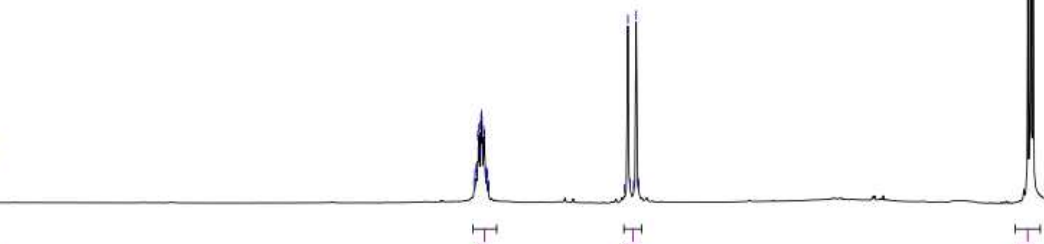

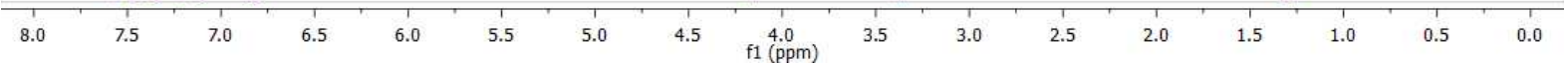

2-cyanophenyl ethyl 4-chloro-3-hydroxybenzylphosphonate

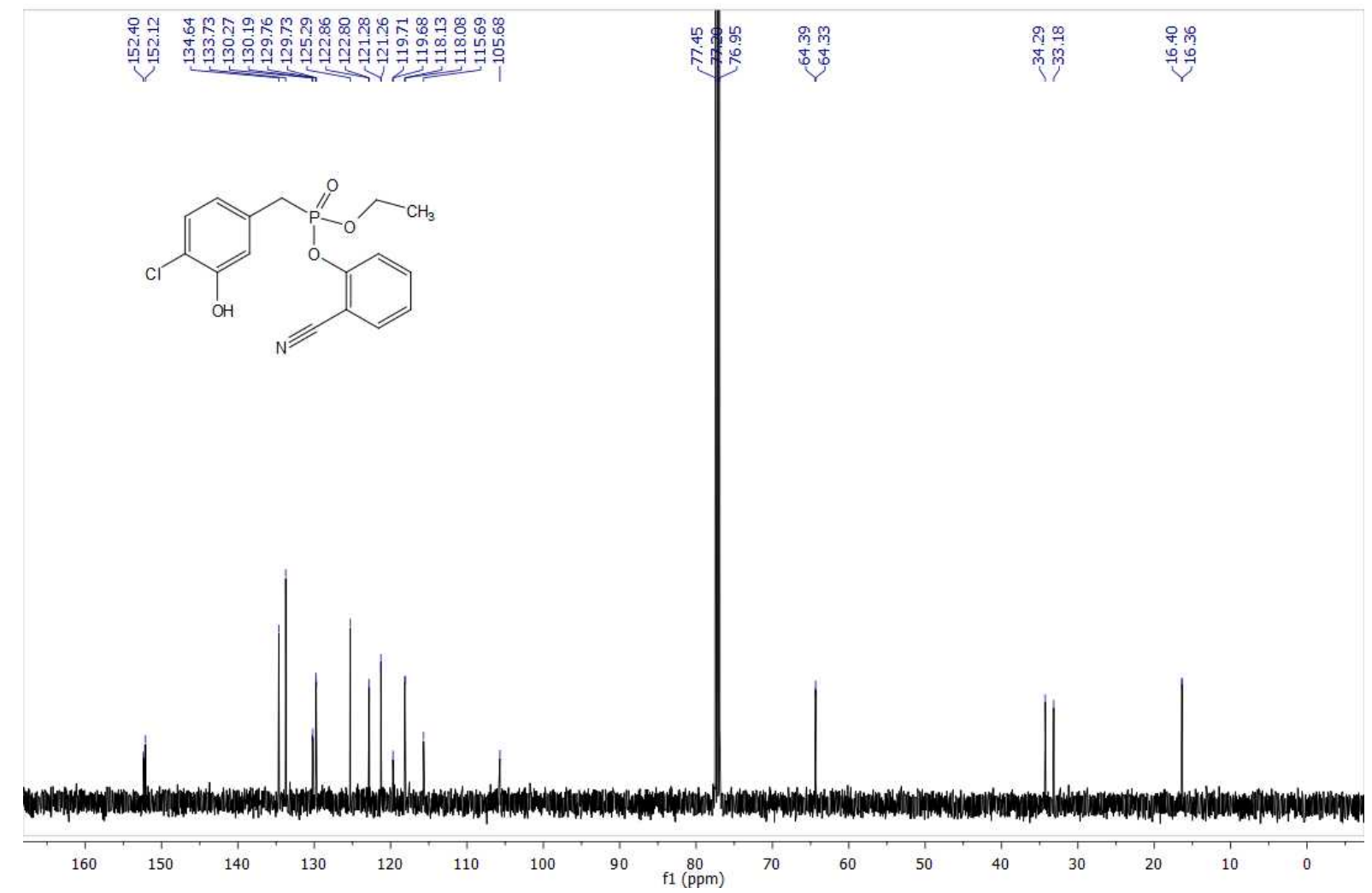


2-cyanophenyl ethyl 4-bromo-3-hydroxybenzylphosphonate (4d):

2-cyanophenyl ethyl 4-bromo-3-hydroxybenzylphosphonate

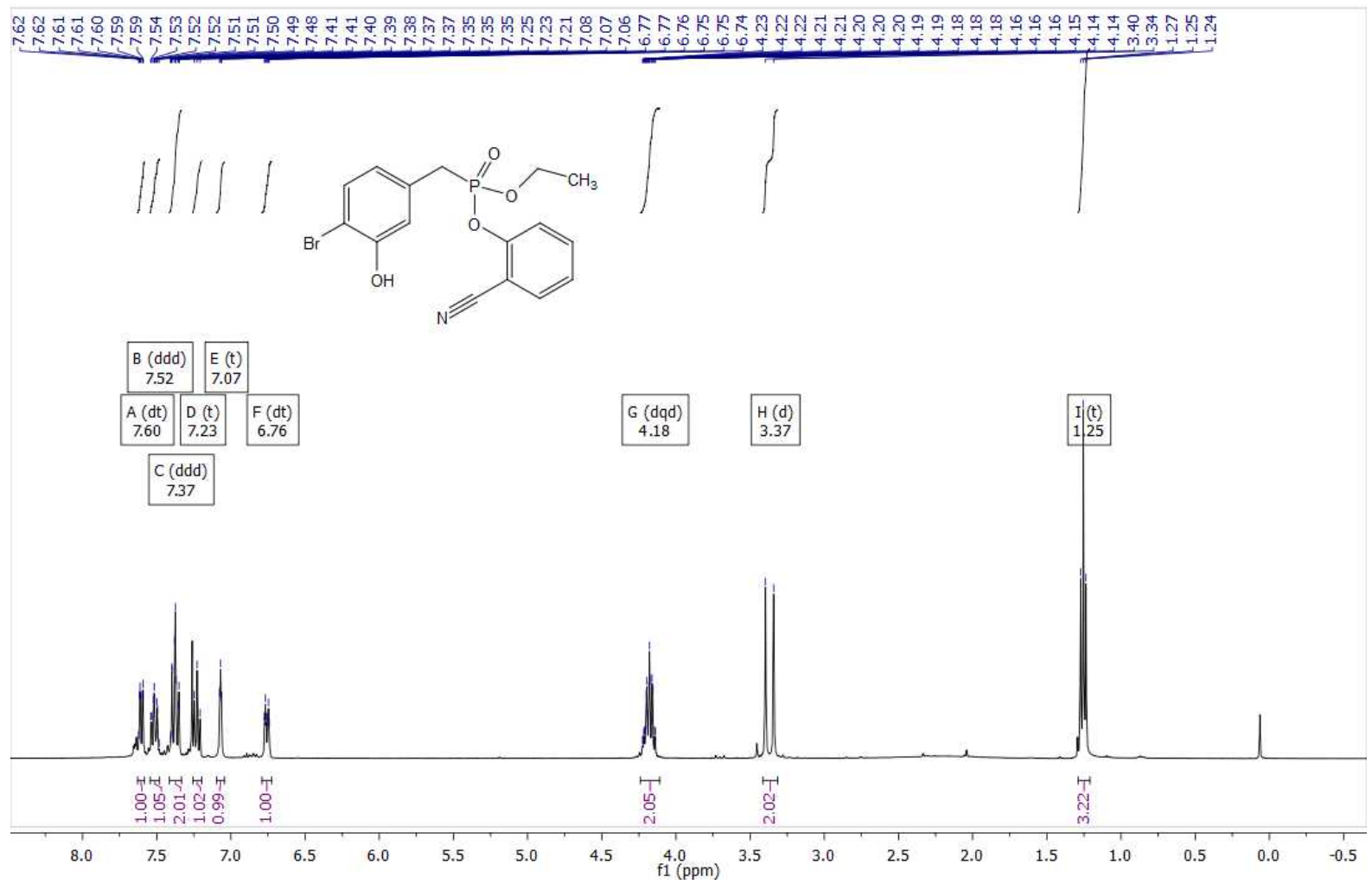

2-cyanophenyl ethyl 4-bromo-3-hydroxybenzylphosphonate

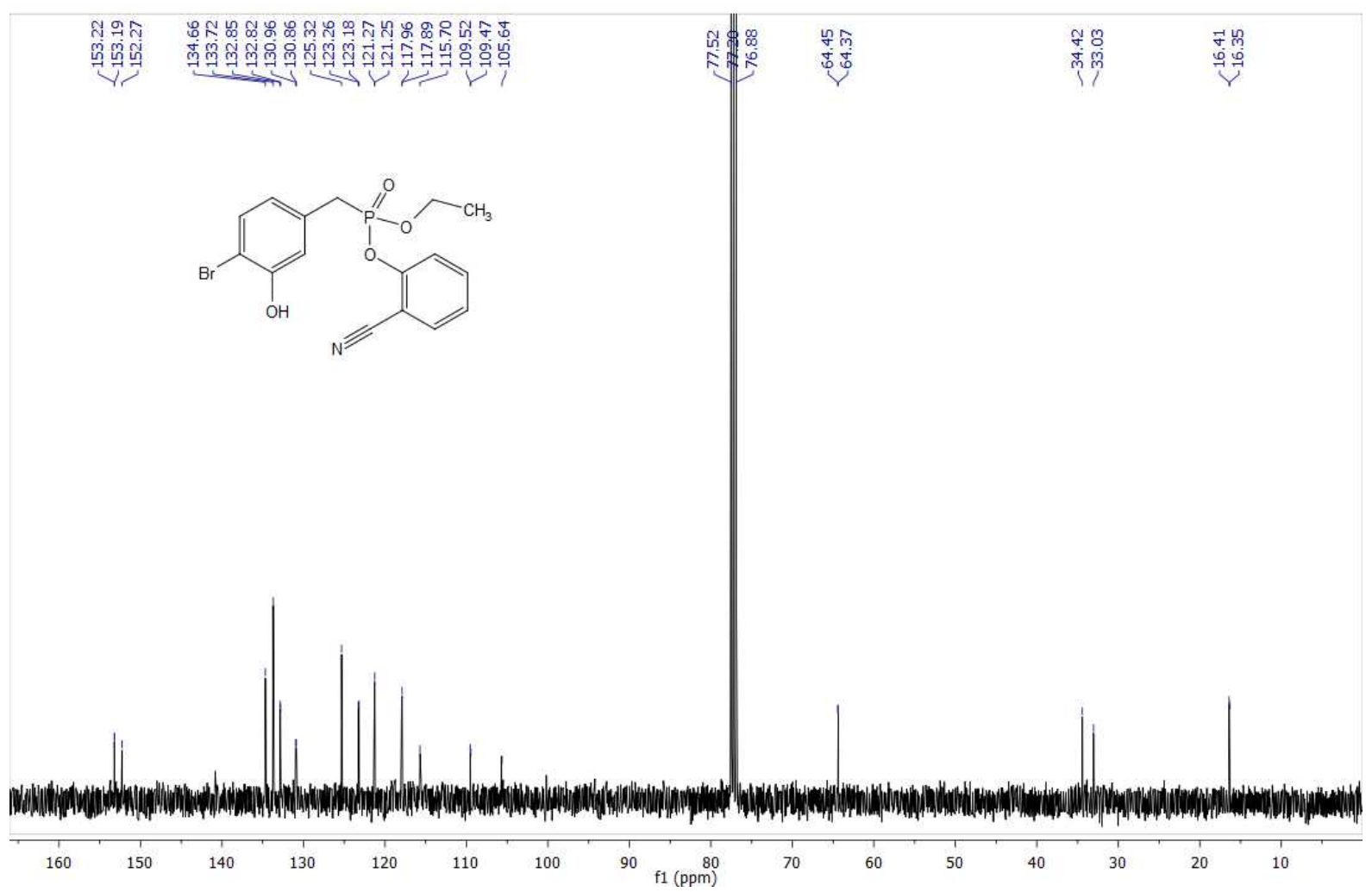


2-cyanophenyl ethyl 2,5-difluoro-3-hydroxybenzylphosphonate (4e):

2-cyanophenyl ethyl 2,5-difluoro-3-hydroxybenzylphosphonate

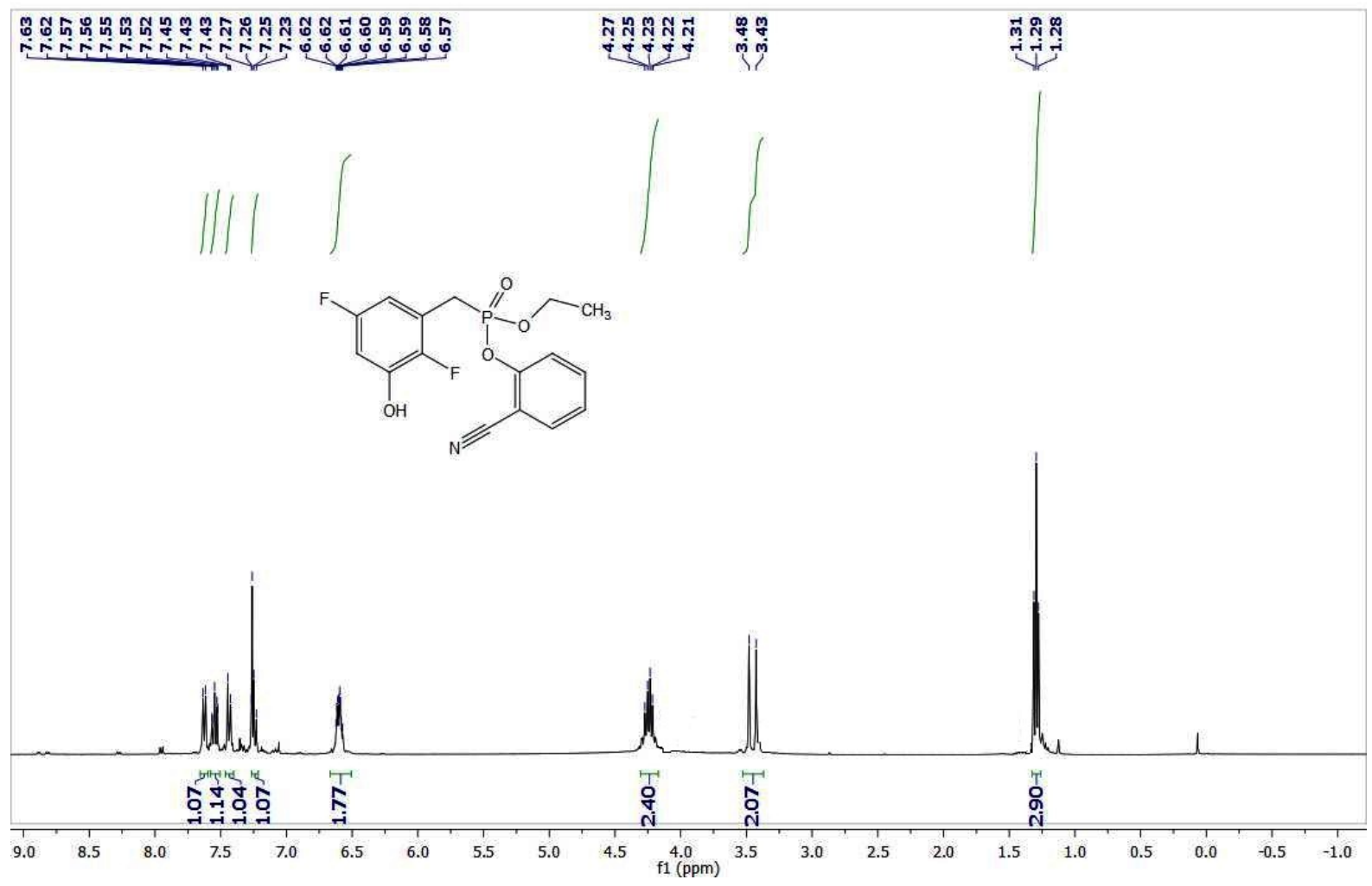

2-cyanophenyl ethyl 2,5-difluoro-3-hydroxybenzylphosphonate

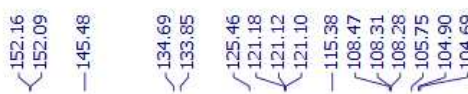<smiles>CCOP(=O)(Cc1cc(F)cc(O)c1F)Oc1ccccc1C#N</smiles>

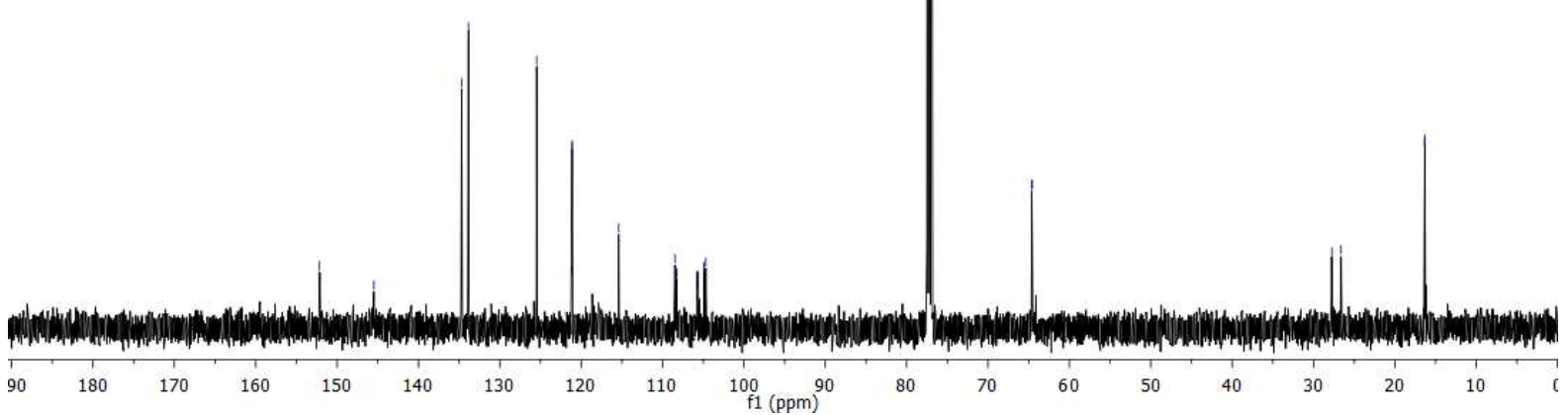


2-cyanophenyl ethyl 3-bromo-5-hydroxybenzylphosphonate (4f):

2-cyanophenyl ethyl 3-bromo-5-hydroxybenzylphosphonate<smiles>CCOP(=O)(Cc1cc(O)cc(Br)c1)Oc1ccccc1C#N</smiles>
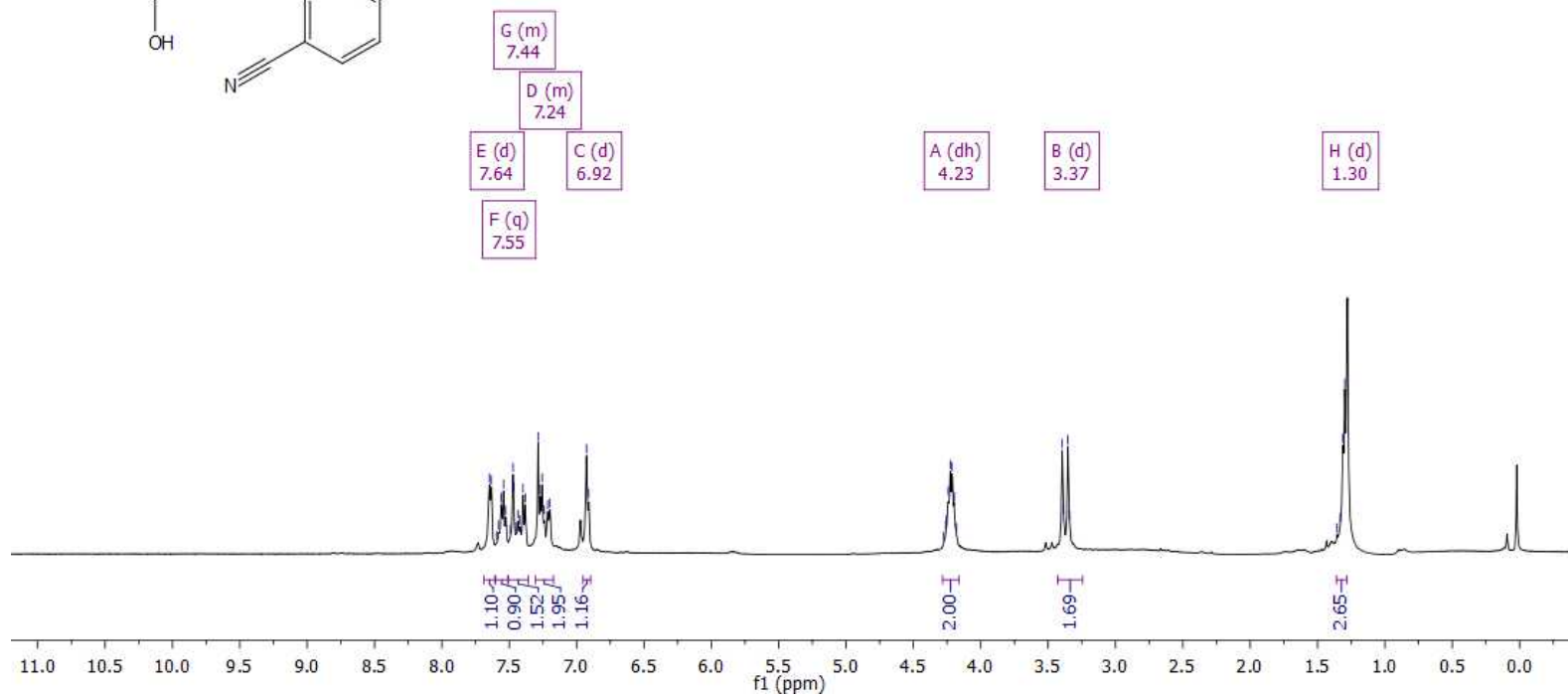

2-cyanophenyl ethyl 3-bromo-5-hydroxybenzylphosphonate

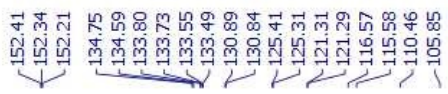<smiles>CCOP(=O)(Cc1cc(O)cc(Br)c1)Oc1ccccc1C#N</smiles>

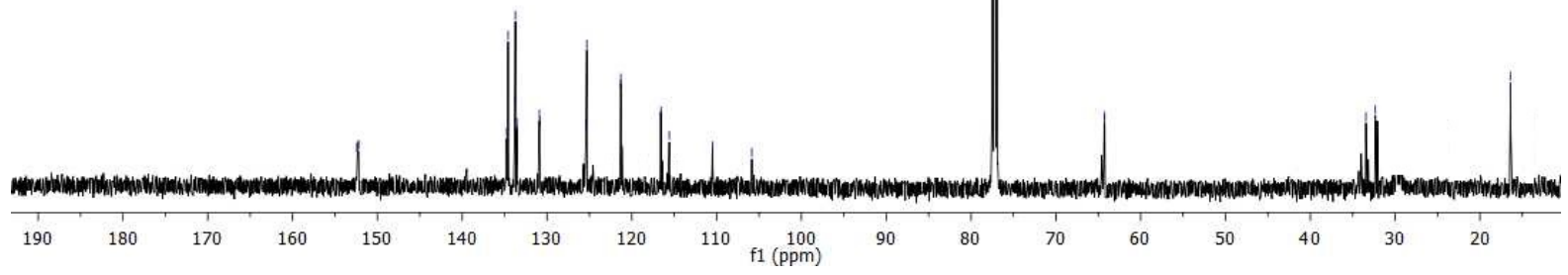


2-cyanophenyl ethyl 3-bromo-4-fluoro-5-hydroxybenzylphosphonate (4g):

2-cyanophenyl ethyl 3-bromo-4-fluoro-5-hydroxybenzylphosphonate

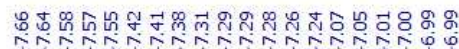

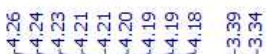

$\sqrt{-7}$<smiles>CCOP(=O)(Cc1cc(O)c(F)c(Br)c1)Oc1ccccc1C#N</smiles>

\begin{tabular}{|c|c|c|}
\hline $\begin{array}{l}D(t) \\
7.57\end{array}$ & & $\begin{array}{l}G \text { (d) } \\
7.06\end{array}$ \\
\hline $\begin{array}{l}C(d) \\
7.65\end{array}$ & $\begin{array}{l}F(\mathrm{~m}) \\
7.28\end{array}$ & \\
\hline & $\begin{array}{l}\text { (d) } \\
7.41\end{array}$ & $\begin{array}{c}H(d d) \\
7.00\end{array}$ \\
\hline
\end{tabular}

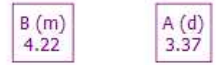

\begin{tabular}{l} 
I (d) \\
1.30 \\
\hline
\end{tabular}

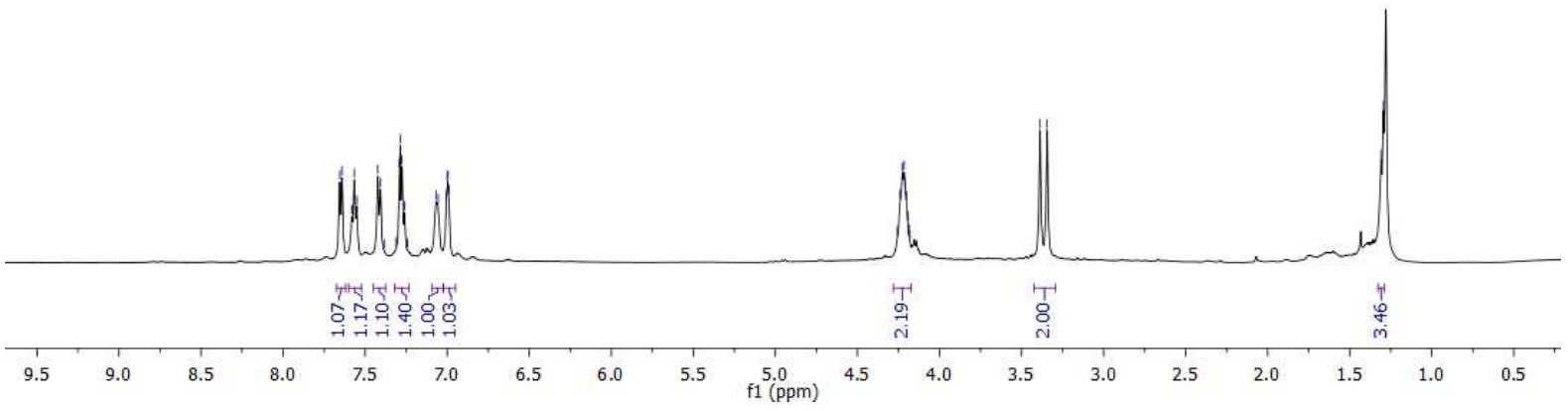

2-cyanophenyl ethyl 3-bromo-4-fluoro-5-hydroxybenzylphosphonate

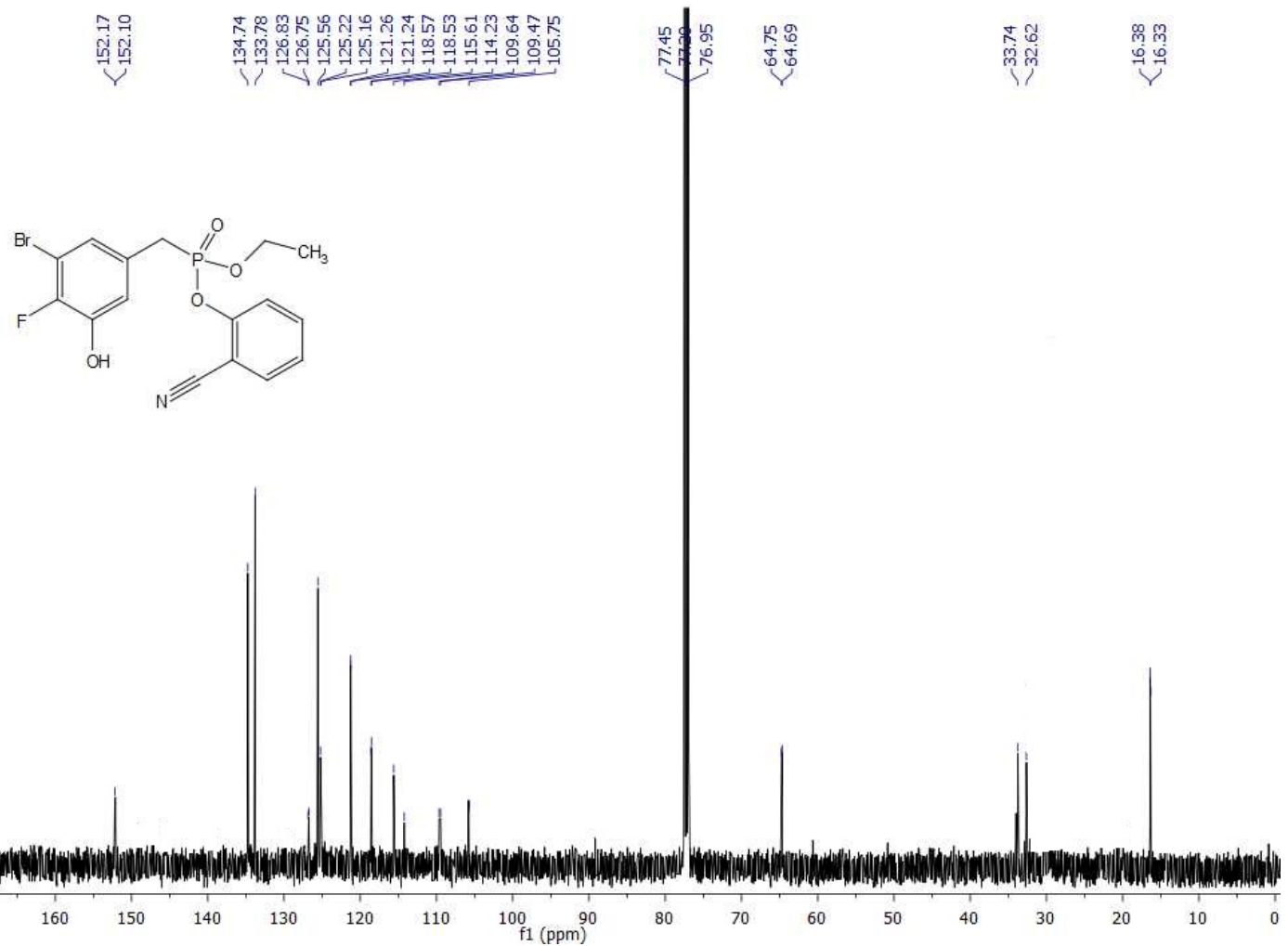




\section{Meta-Acetoxylation:}

3-(((2-cyanophenoxy)(ethoxy)phosphoryl)methyl)phenyl acetate (5a):

3-(((2-cyanophenoxy)(ethoxy)phosphoryl)methyl)phenyl acetate

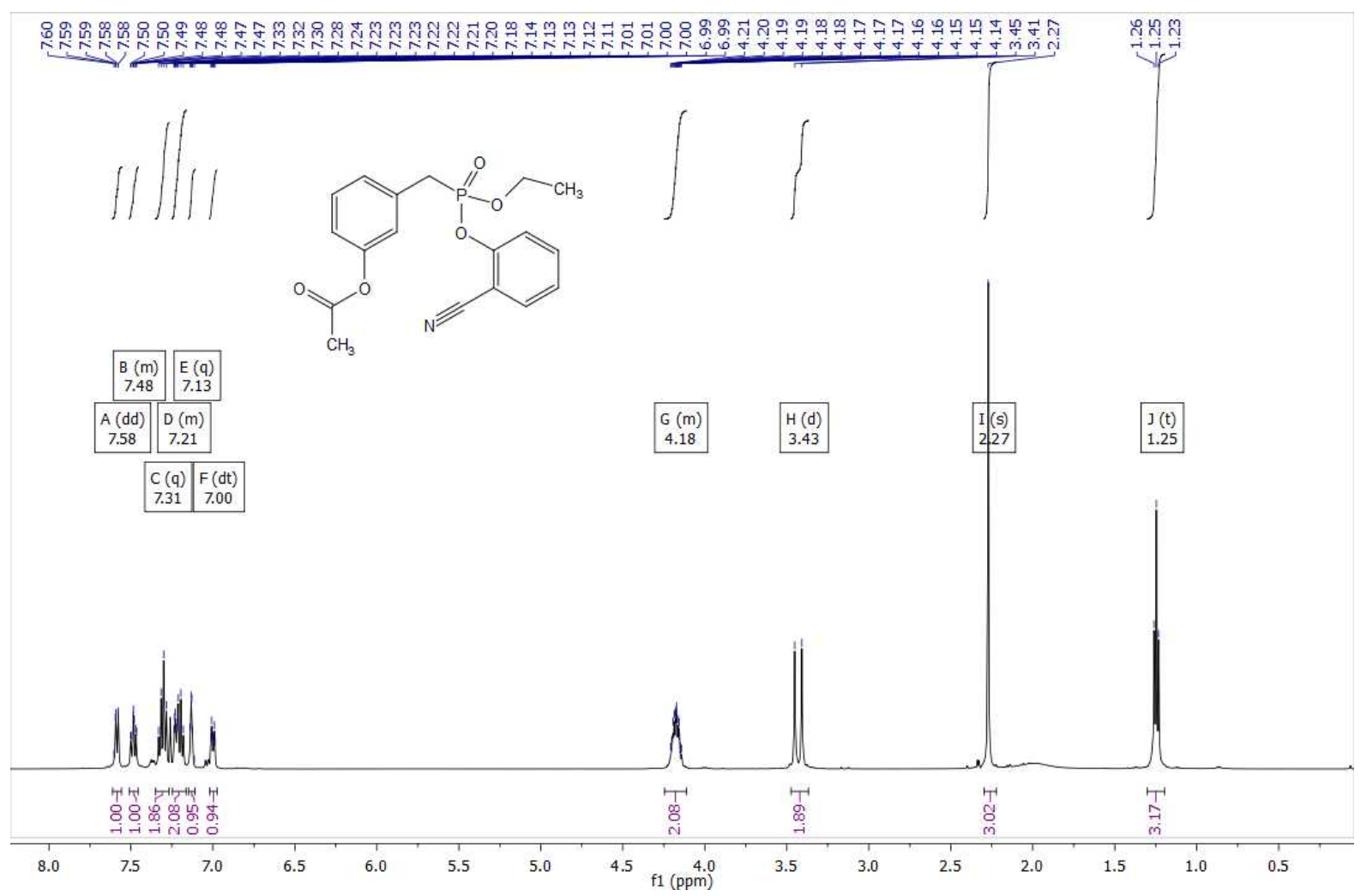

3-(((2-cyanophenoxy)(ethoxy)phosphoryl)methyl)phenyl acetate

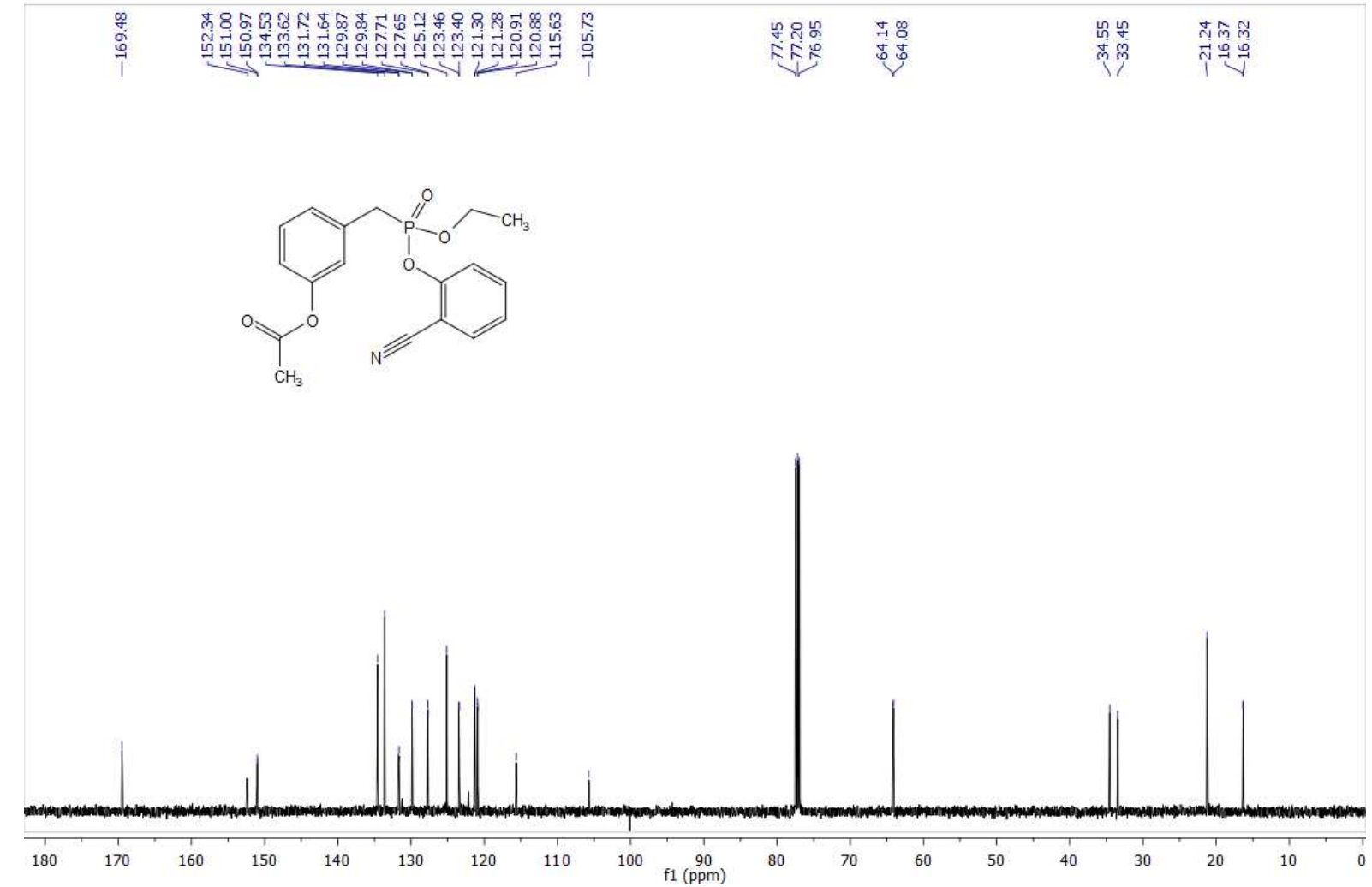


5-(((2-cyanophenoxy)(ethoxy)phosphoryl)methyl)-2-methylphenyl acetate (5b):

5-(((2-cyanophenoxy)(ethoxy)phosphoryl)methyl)-2-methylphenyl acetate

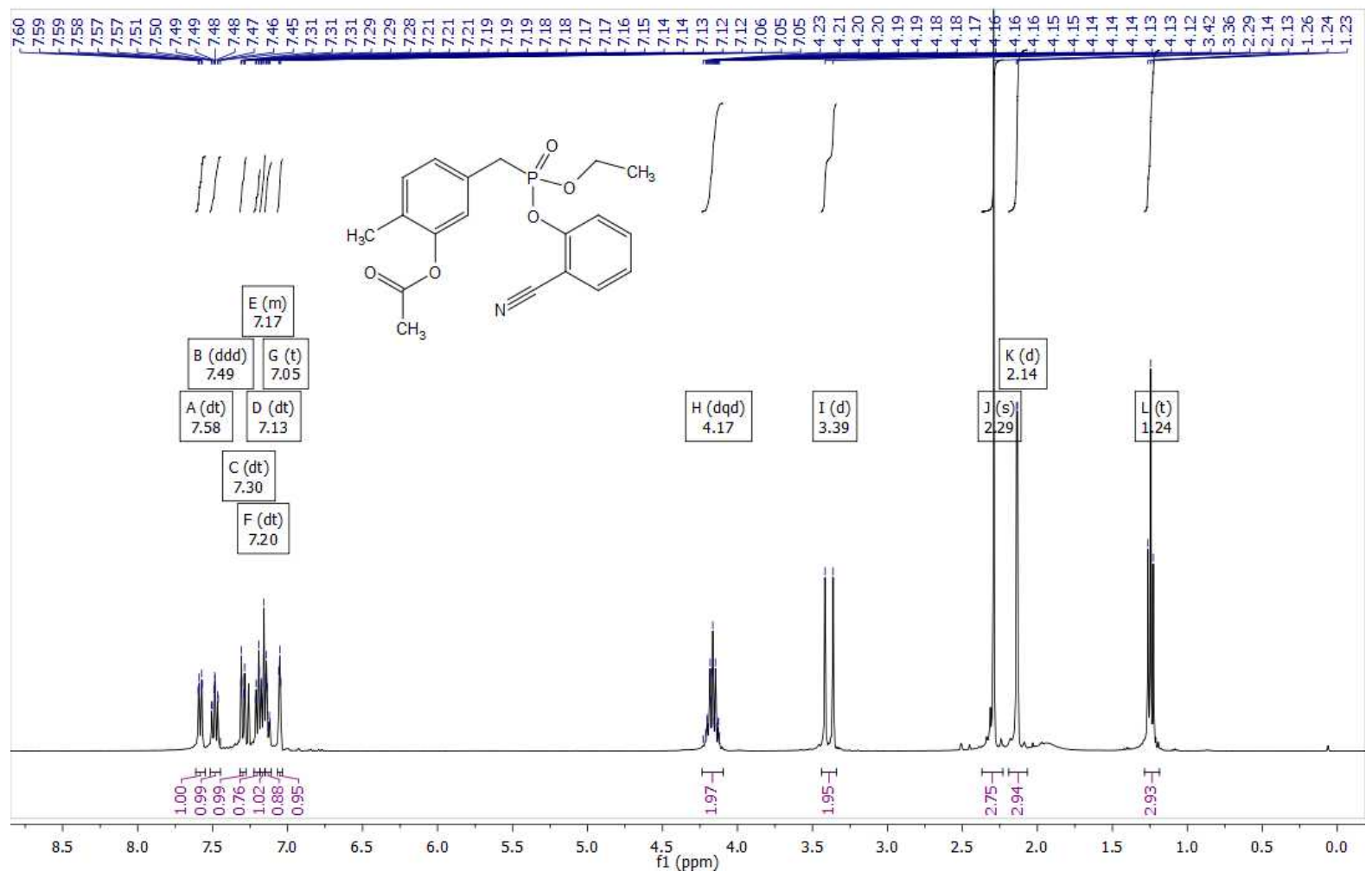

5-(((2-cyanophenoxy)(ethoxy)phosphoryl)methyl)-2-methylphenyl acetate

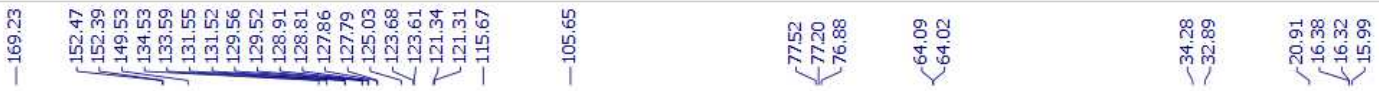<smiles>CCOP(=O)(Cc1ccc(C)c(OC(C)=O)c1)Oc1ccccc1C#N</smiles>

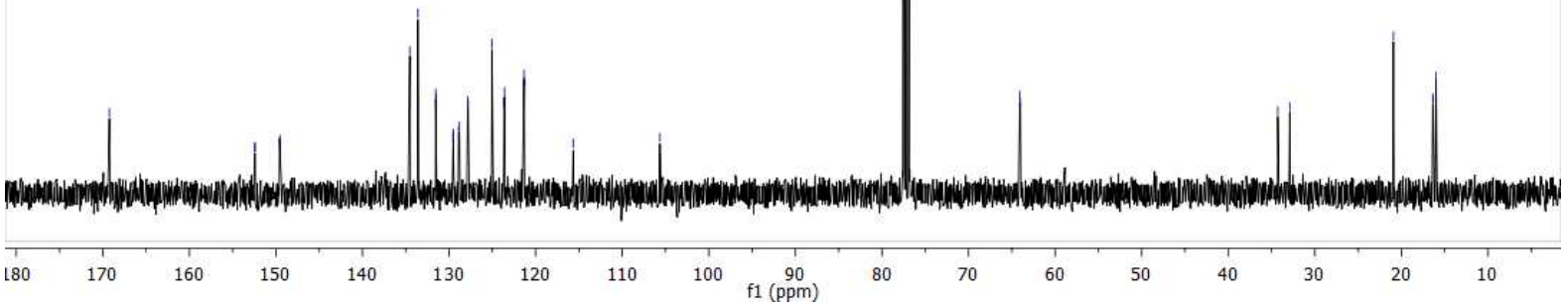


5-(((2-cyanophenoxy)(ethoxy)phosphoryl)methyl)-2-methoxyphenyl acetate (5c):

5-(((2-cyanophenoxy)(ethoxy)phosphoryl)methyl)-2-methoxyphenyl acetate
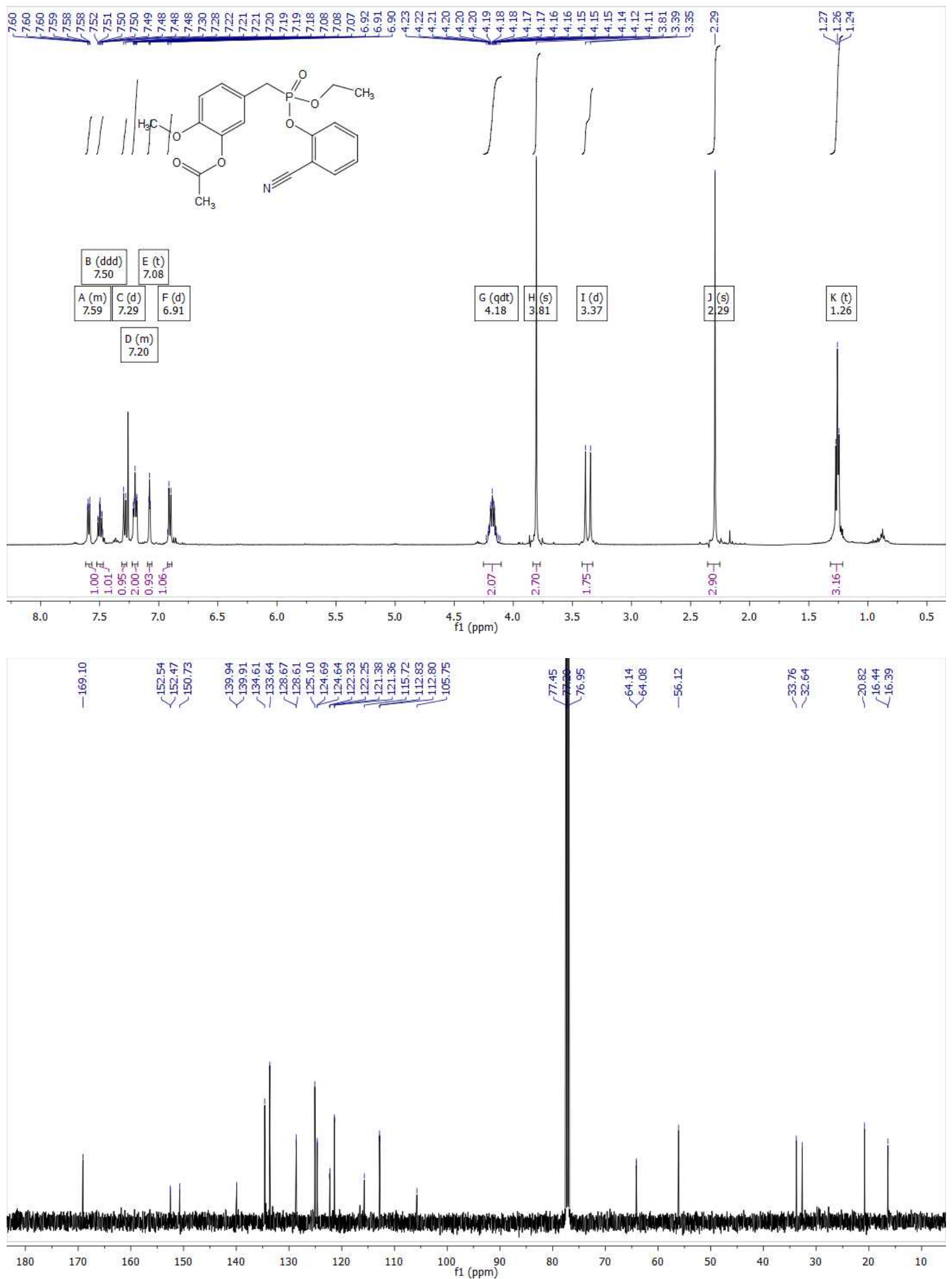
2-bromo-5-(((2-cyanophenoxy)(ethoxy)phosphoryl)methyl)phenyl acetate (5d):

2-bromo-5-(((2-cyanophenoxy)(ethoxy)phosphoryl)methyl)phenyl acetate

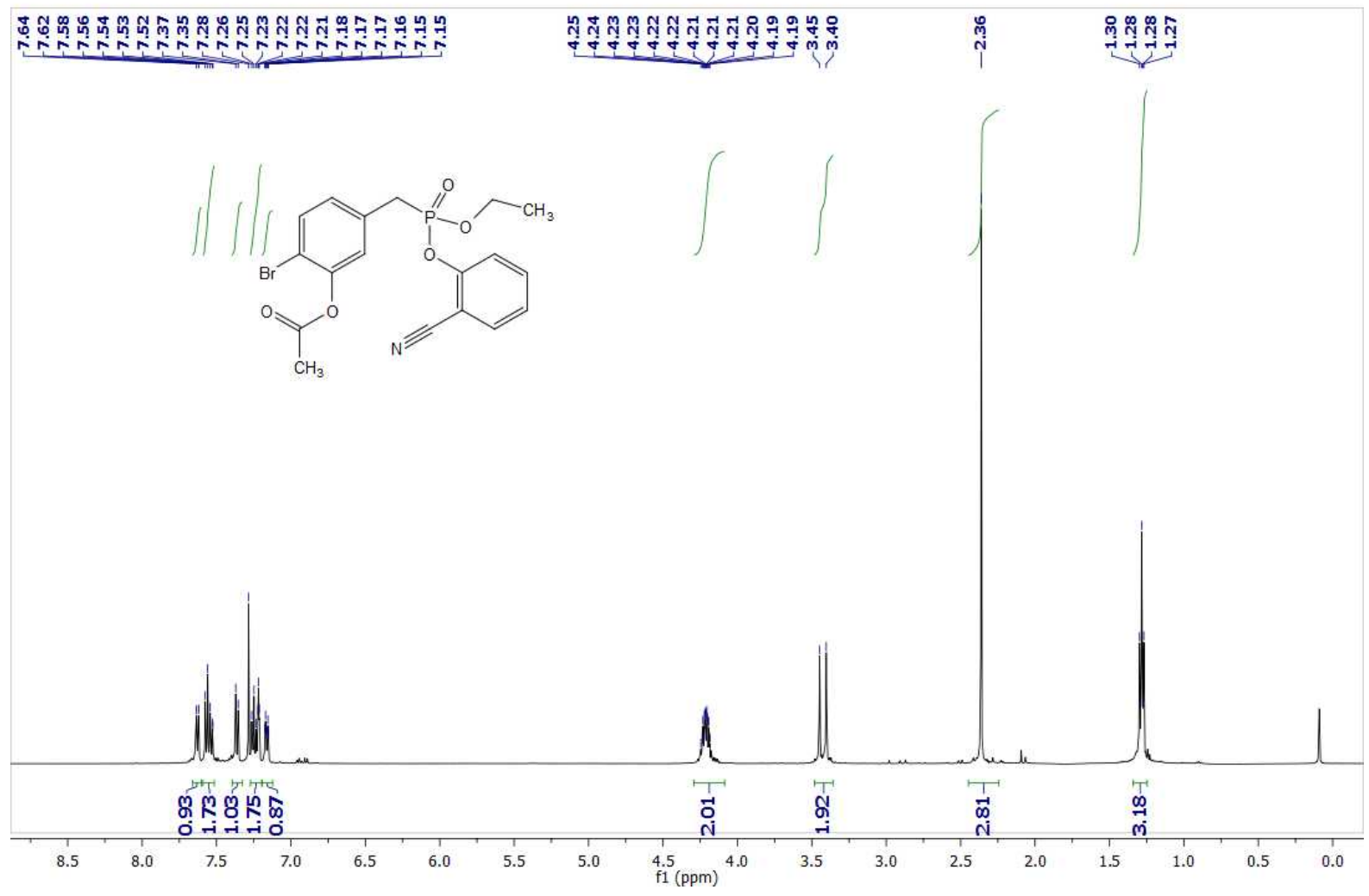

2-bromo-5-(((2-cyanophenoxy)(ethoxy)phosphoryl)methyl)phenyl acetate

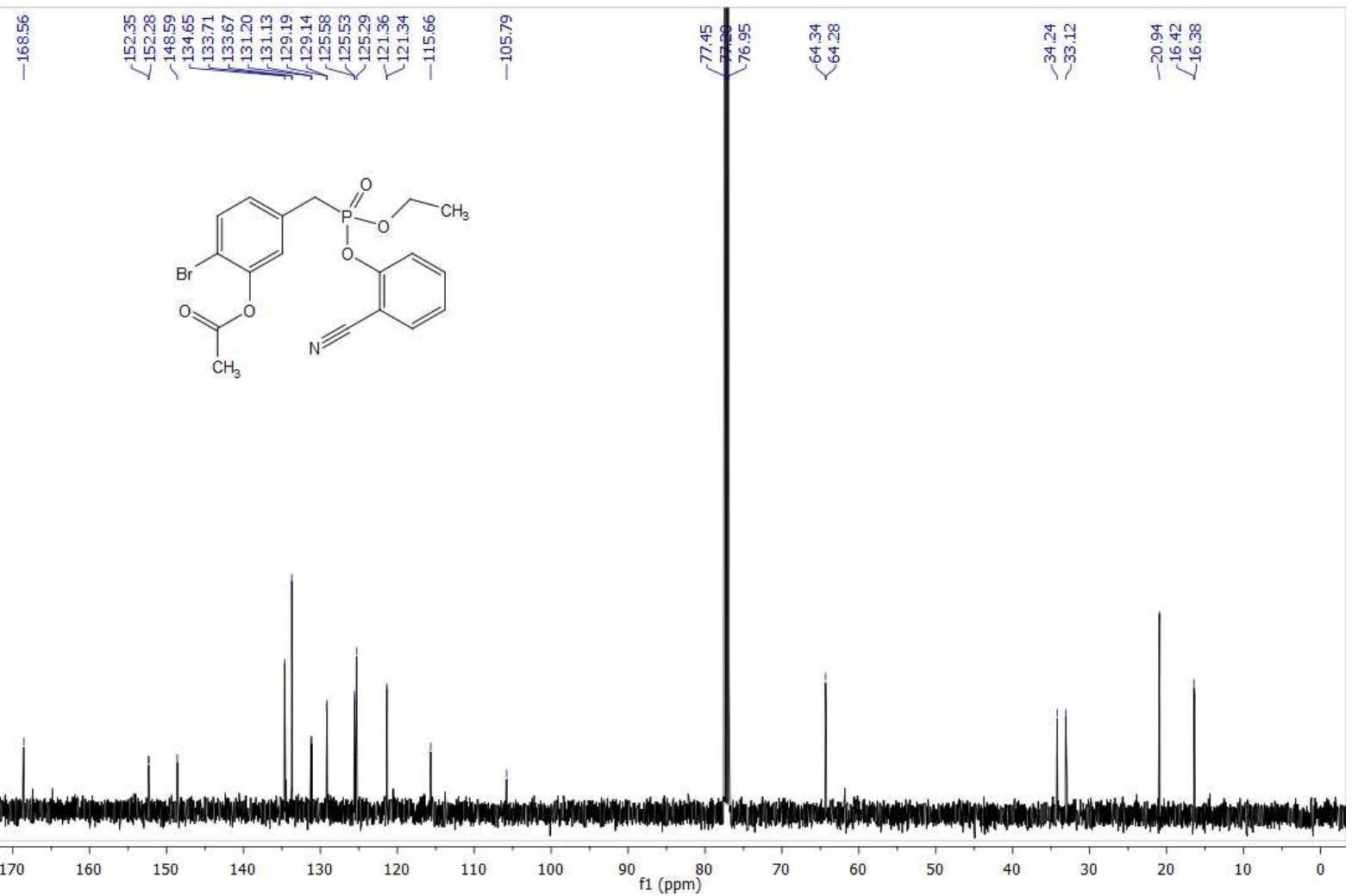


2-chloro-5-(((2-cyanophenoxy)(ethoxy)phosphoryl)methyl)phenyl acetate (5e):

2-chloro-5-(((2-cyanophenoxy)(ethoxy)phosphoryl)methyl)phenyl acetate

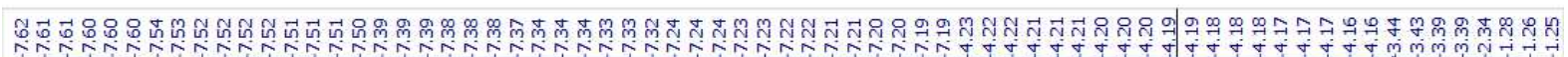

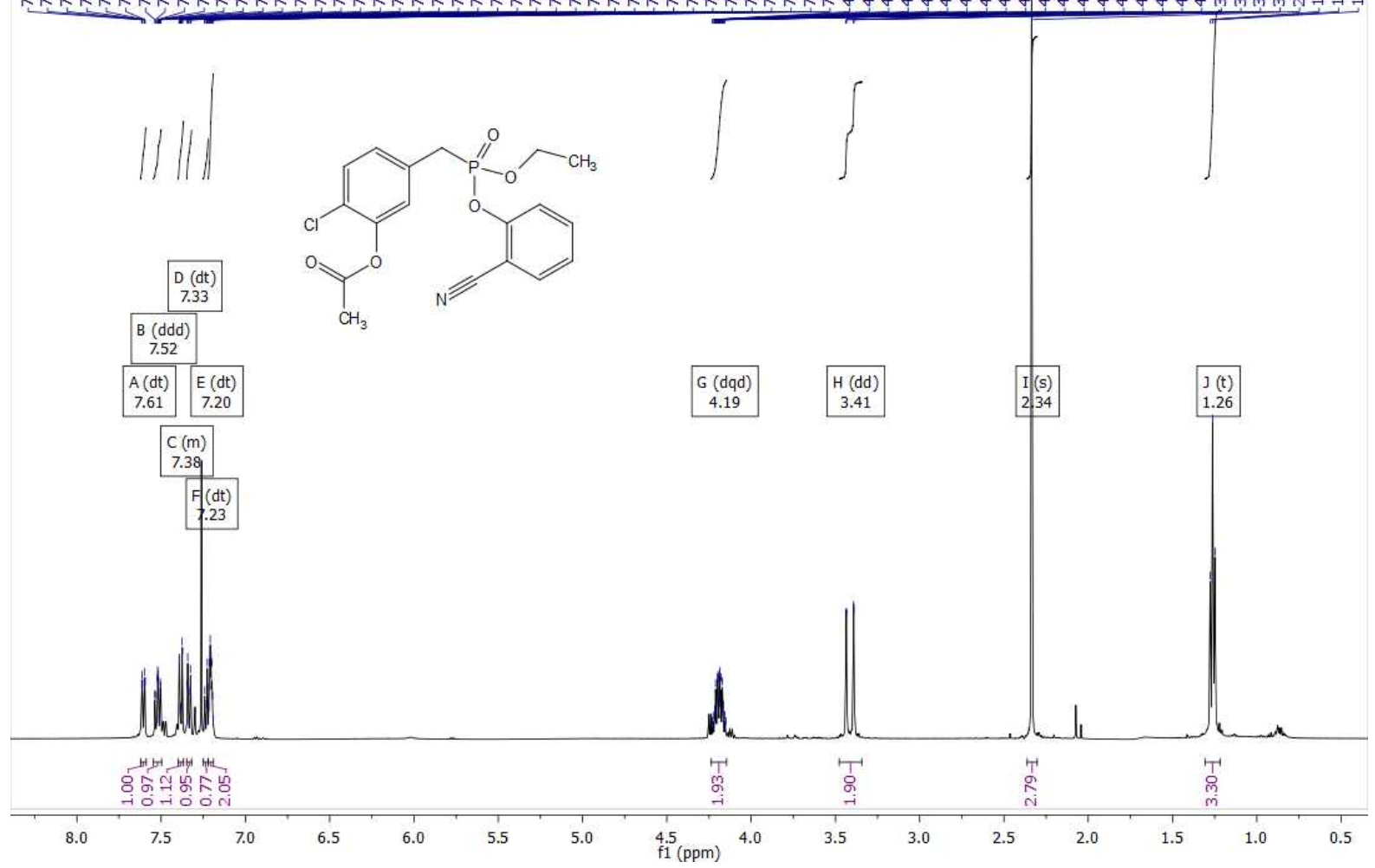

2-chloro-5-(((2-cyanophenoxy)(ethoxy)phosphoryl)methyl)phenyl acetate

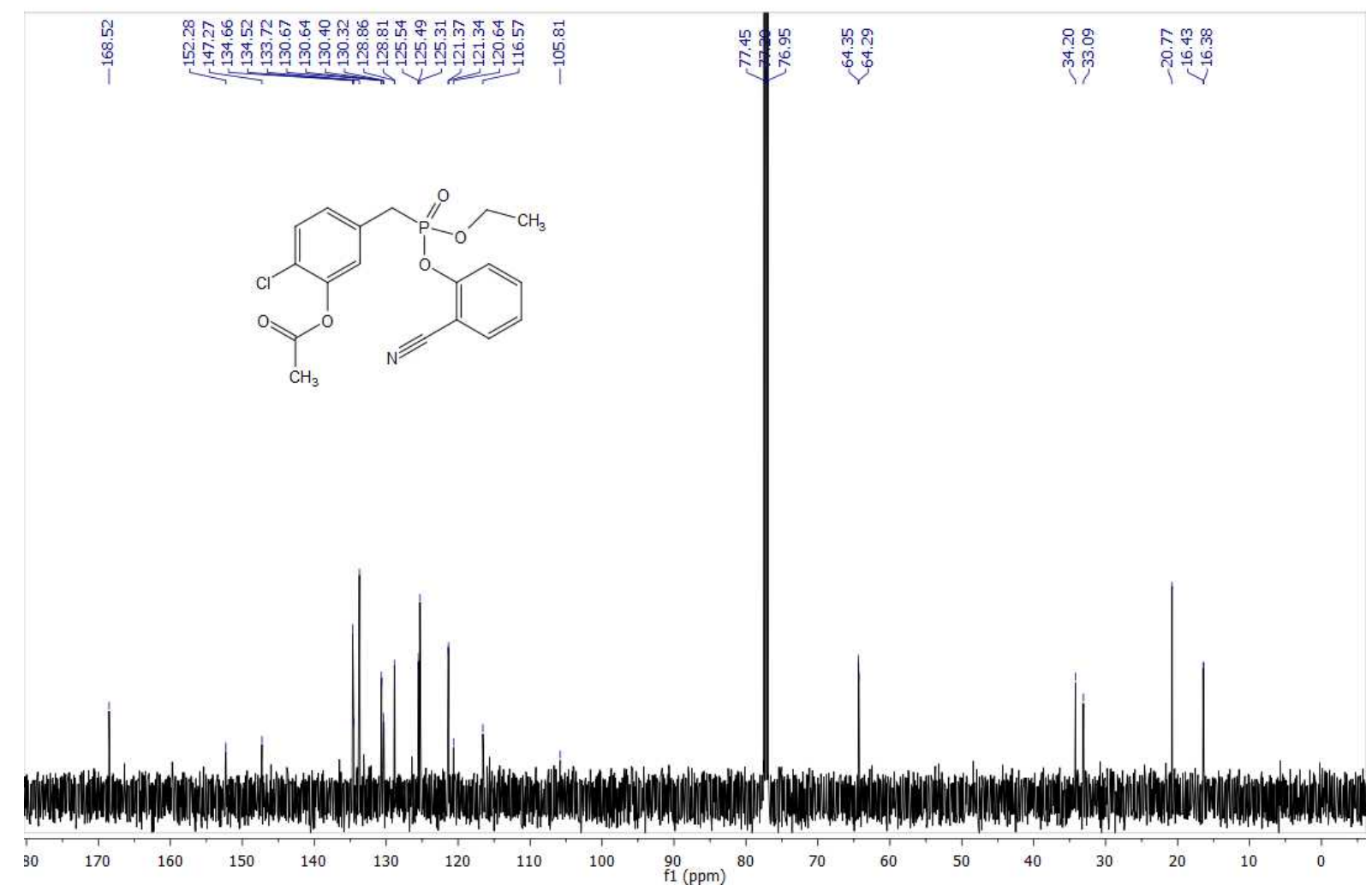


3-bromo-5-(((2-cyanophenoxy)(ethoxy)phosphoryl)methyl)-2-fluorophenyl acetate (5f):

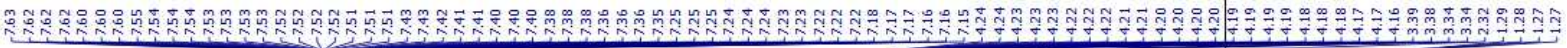
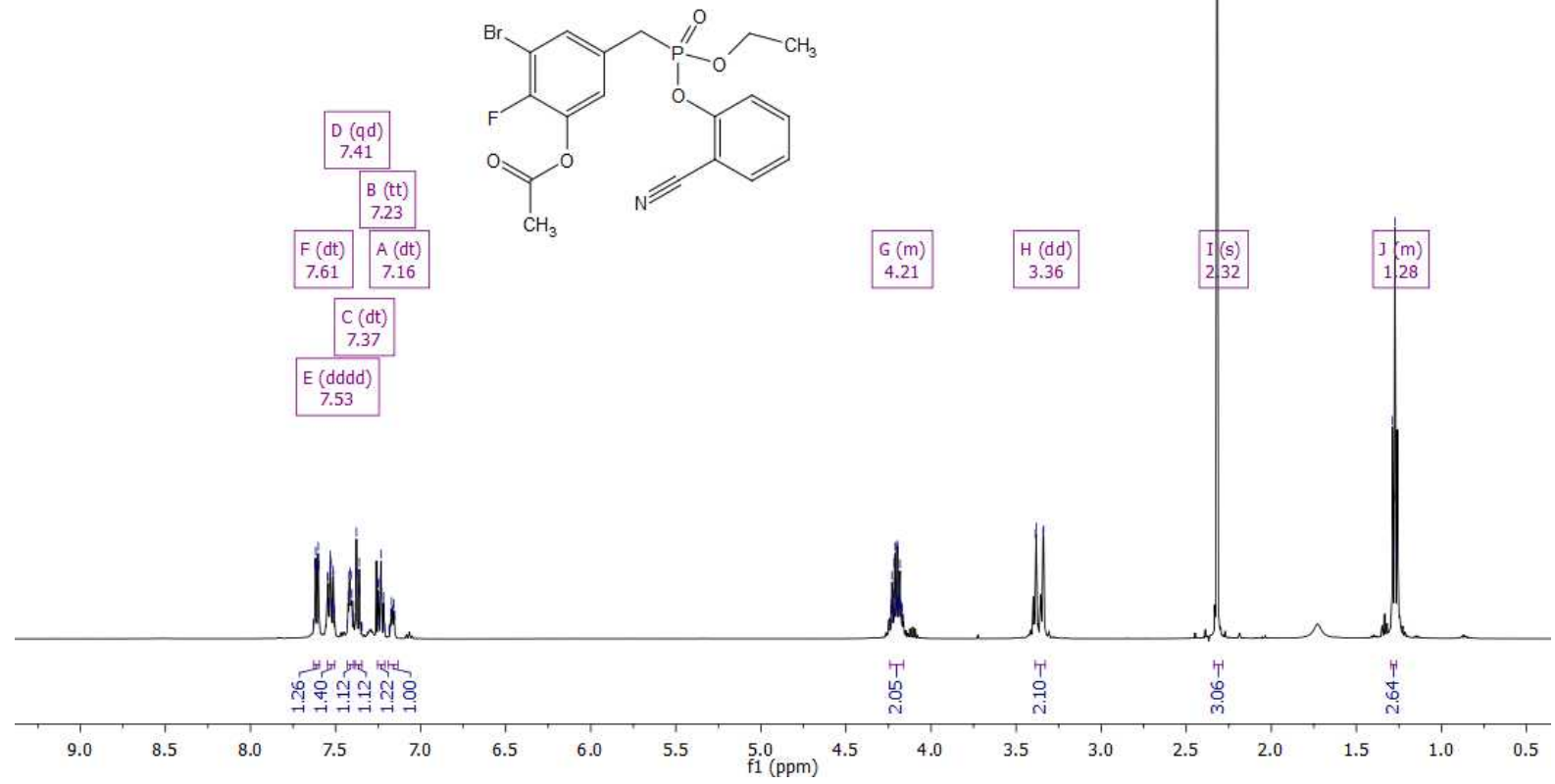

3-bromo-5-(((2-cyanophenoxy)(ethoxy)phosphoryl)methyl)-2-fluorophenyl acetate

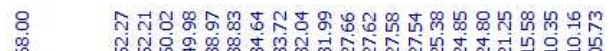

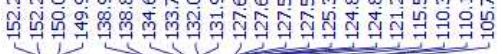

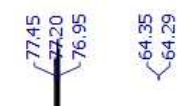

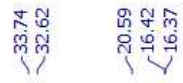<smiles>CCOP(=O)(Cc1cc(Br)c(F)c(OC(C)=O)c1)Oc1ccccc1C#N</smiles>

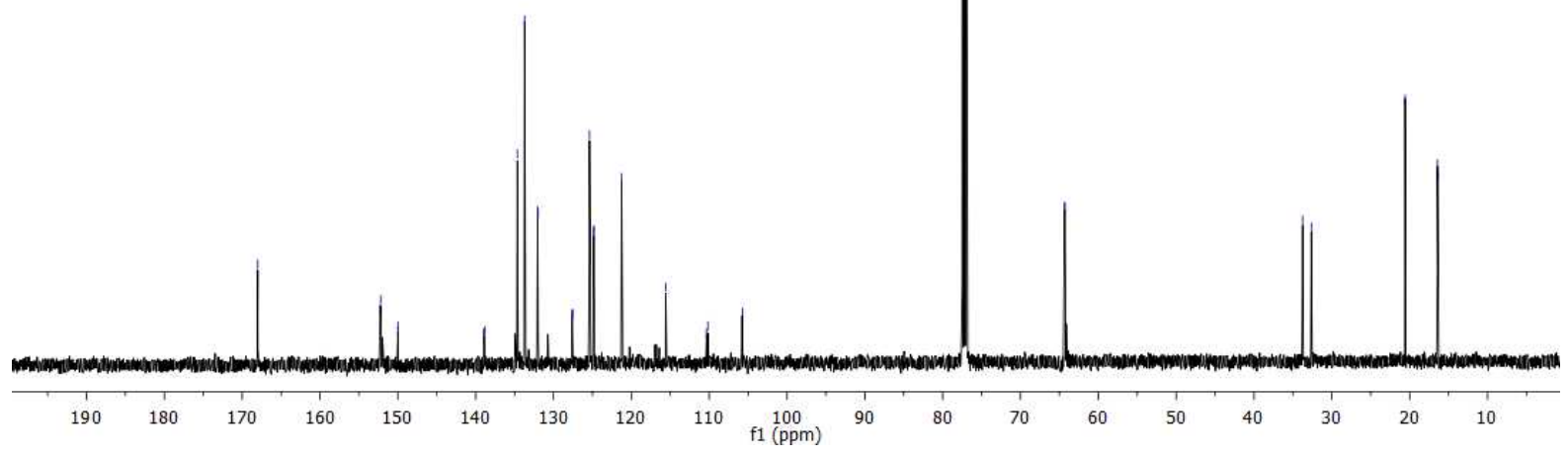


31P NMR:

กู

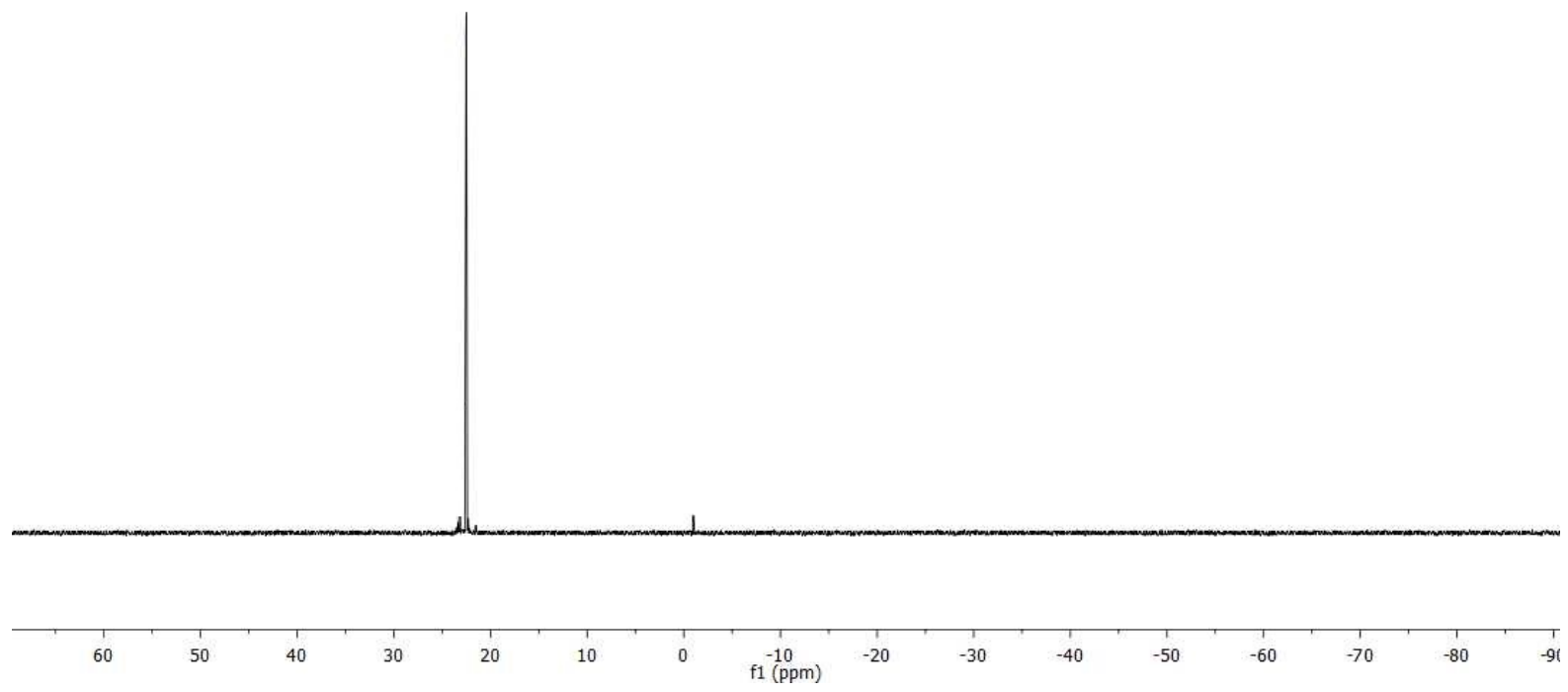


(E)-ethyl 3-(3-styrylphenyl)acrylate (6a):
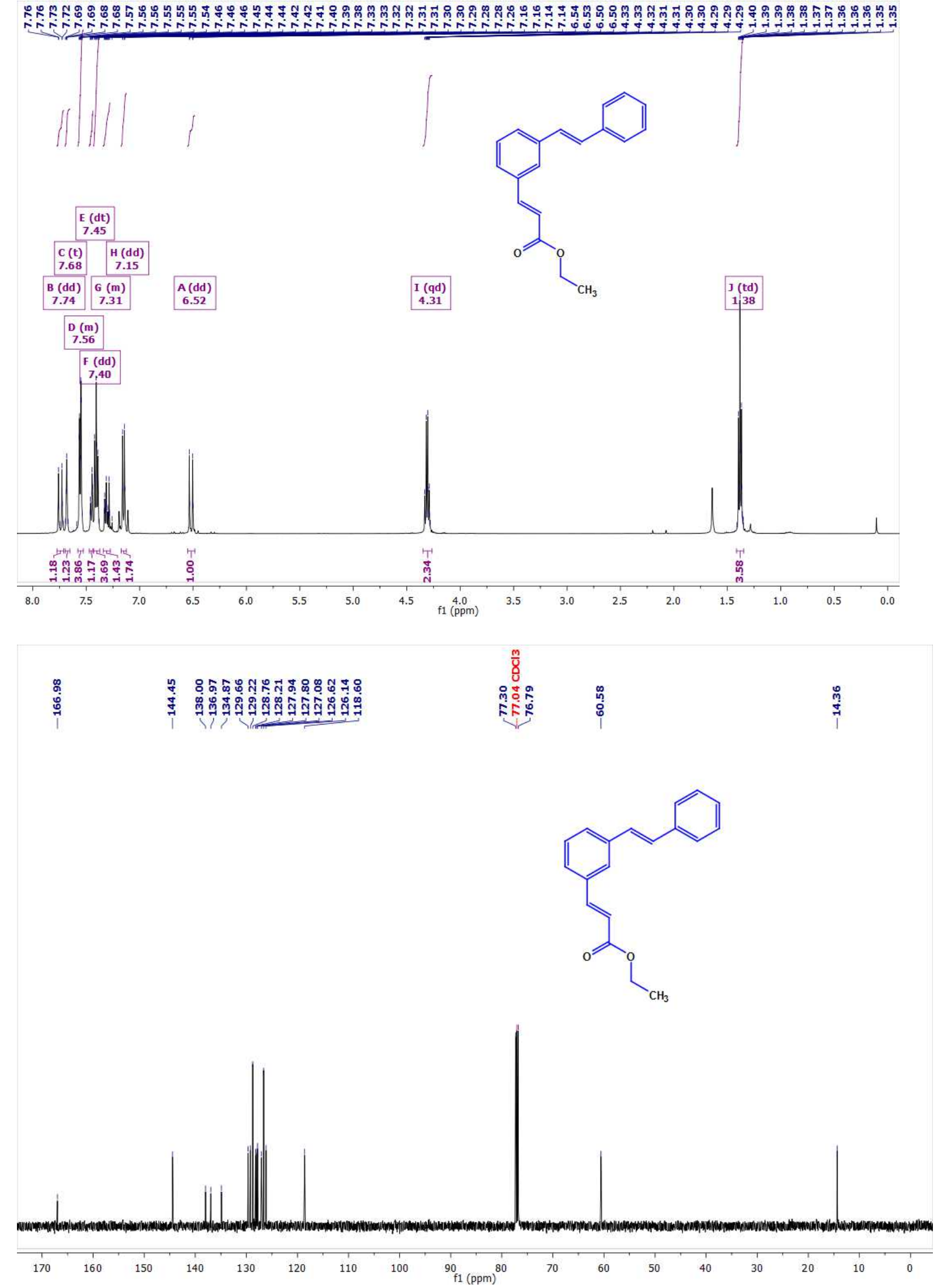
(E)-3-styrylphenyl acetate (6b):

(E)-3-styrylphenyl acetate

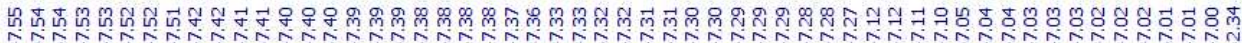
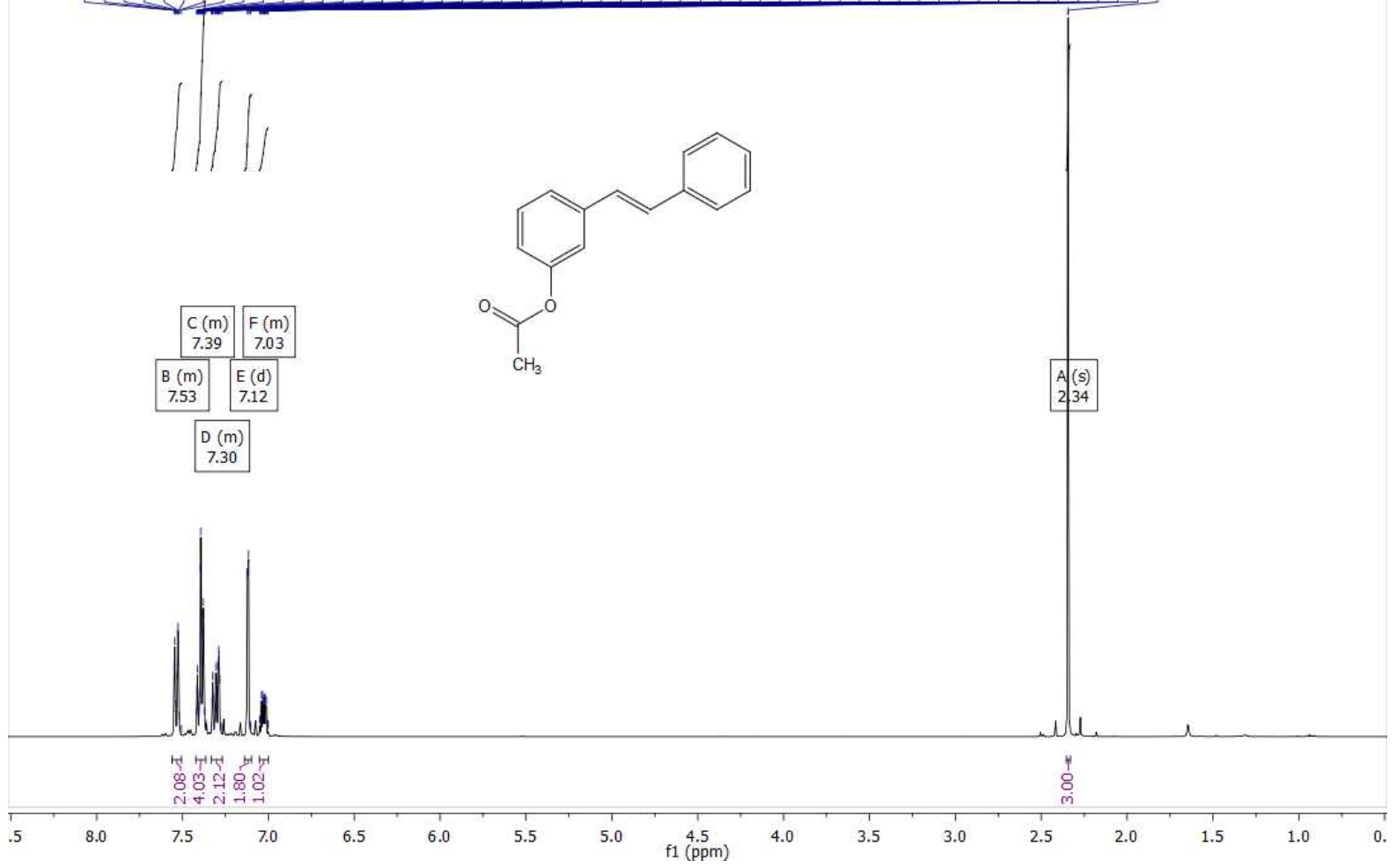

(E)-3-styrylphenyl acetate
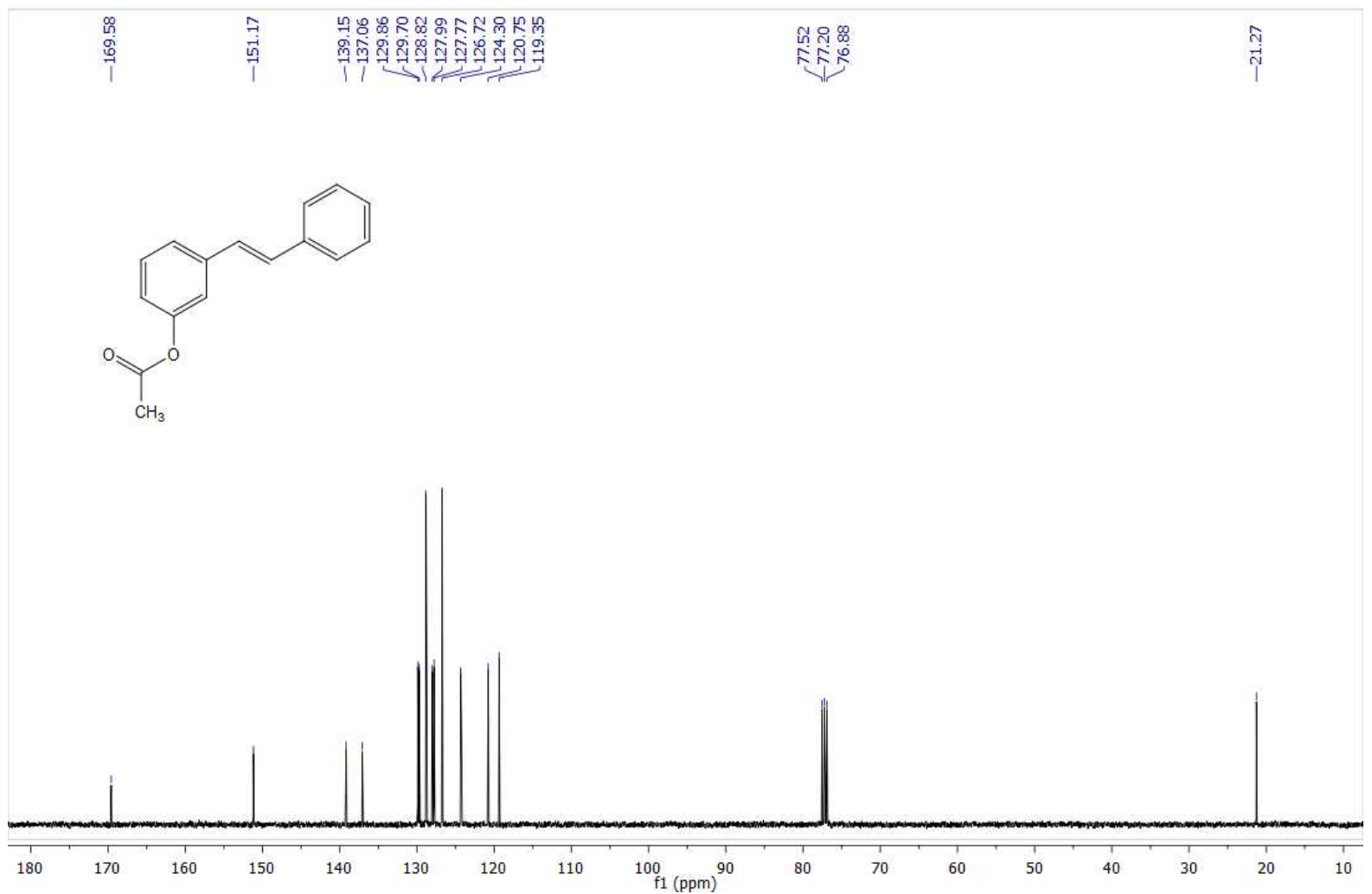
(E)-3-styrylphenol (6c):

(E)-3-styrylphenol

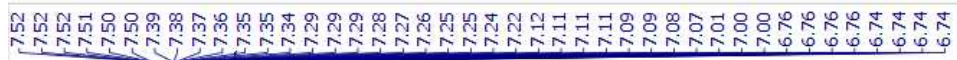

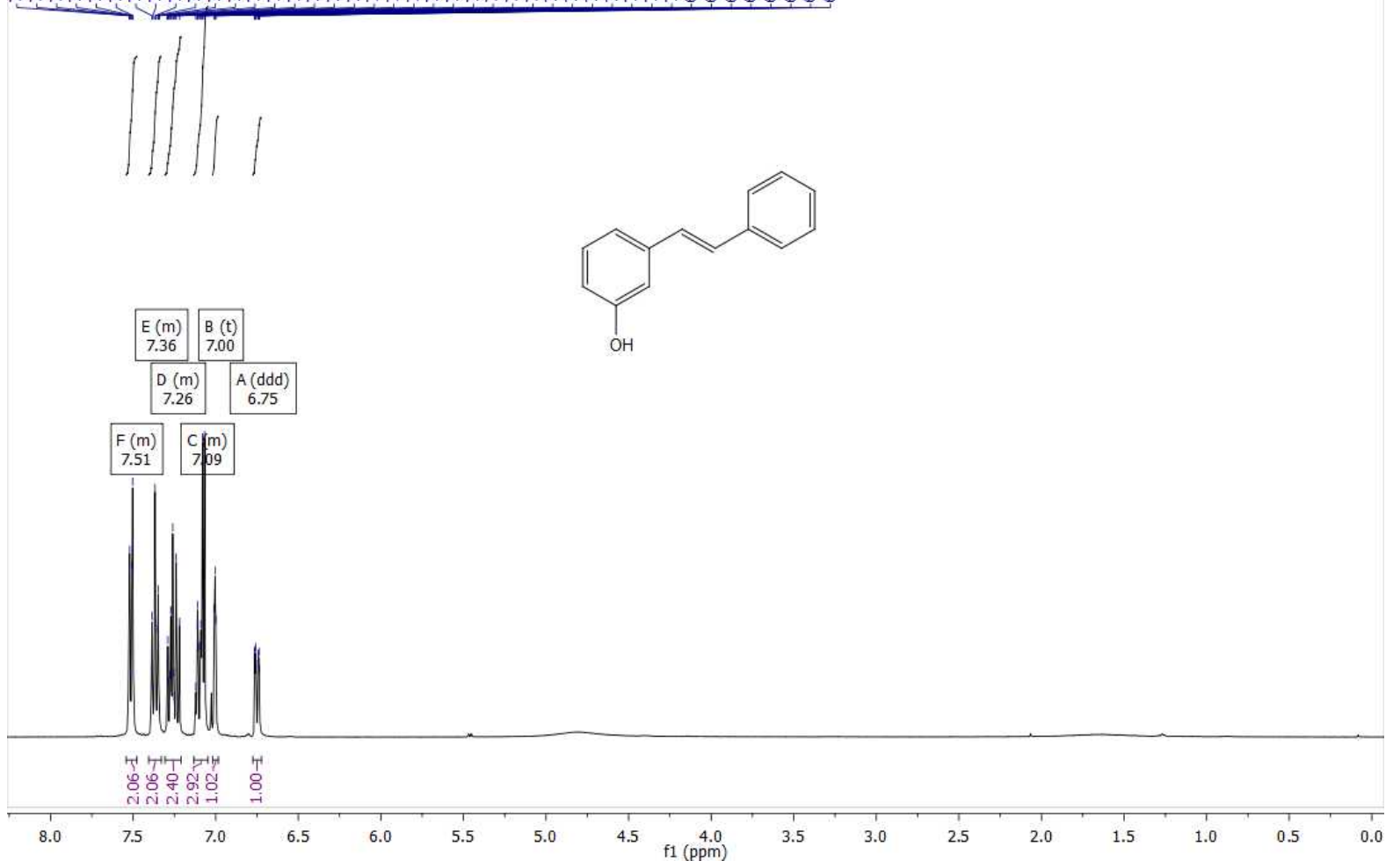

(E)-3-styrylphenol

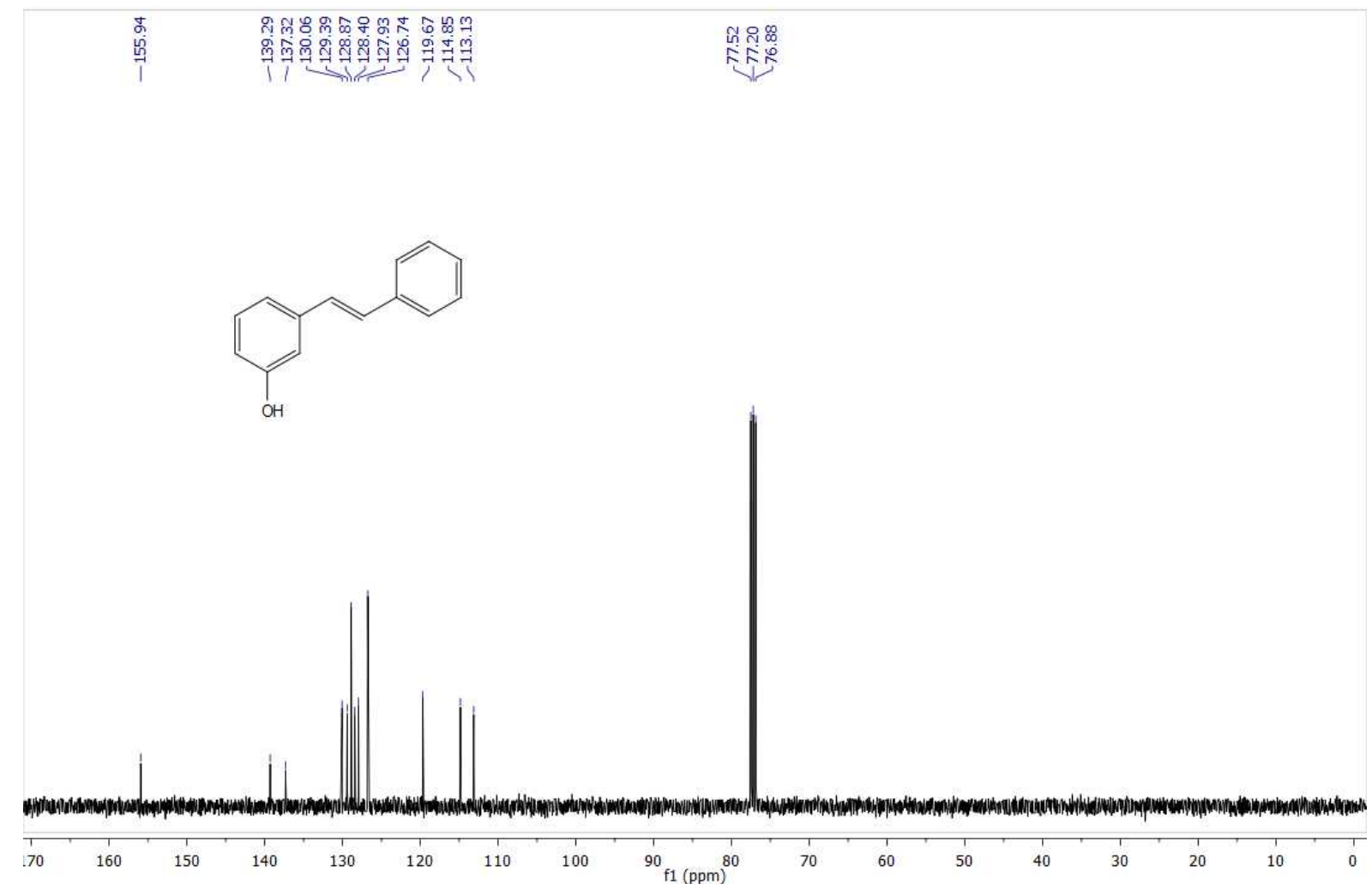


(2E,2'E)-diethyl 3,3'-(5-styryl-1,3-phenylene)diacrylate (6d):

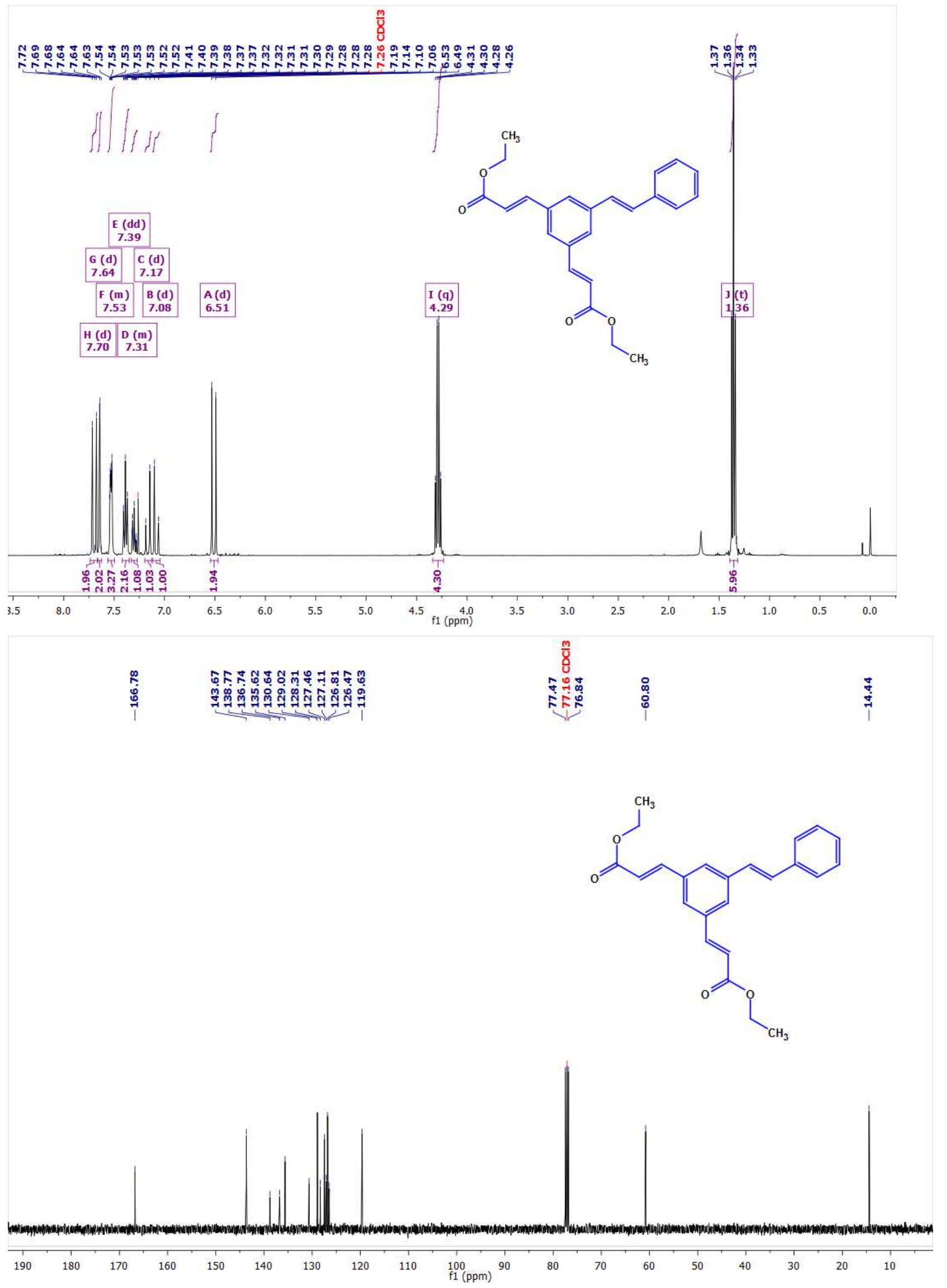


E)-ethyl 3-(3-((E)-3-oxobut-1-enyl)-5-styrylphenyl)acrylate (6e):

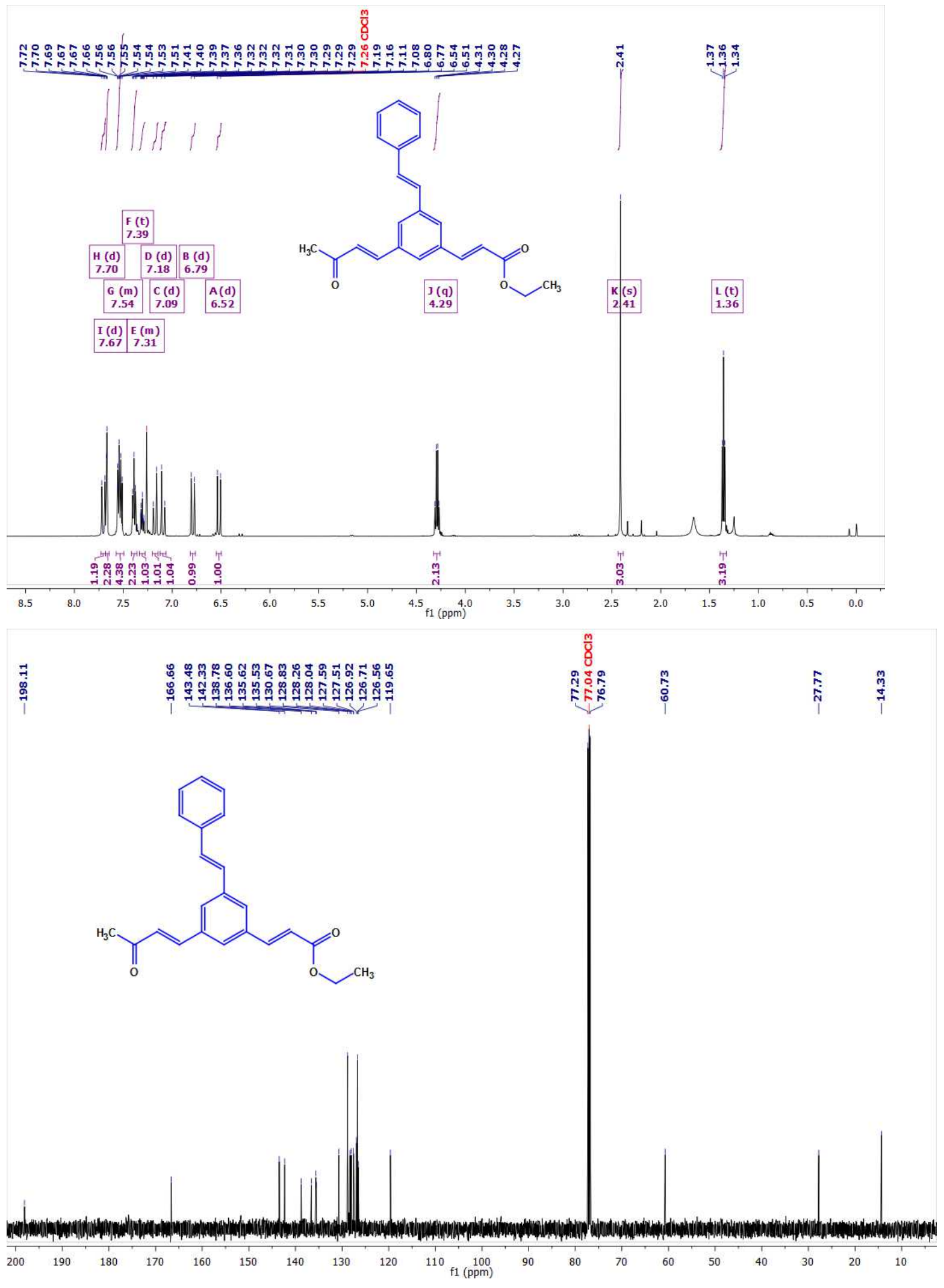


(E)-3-styrylphenyl trifluoromethanesulfonate (7):

(E)-3-styrylphenyl trifluoromethanesulfonate

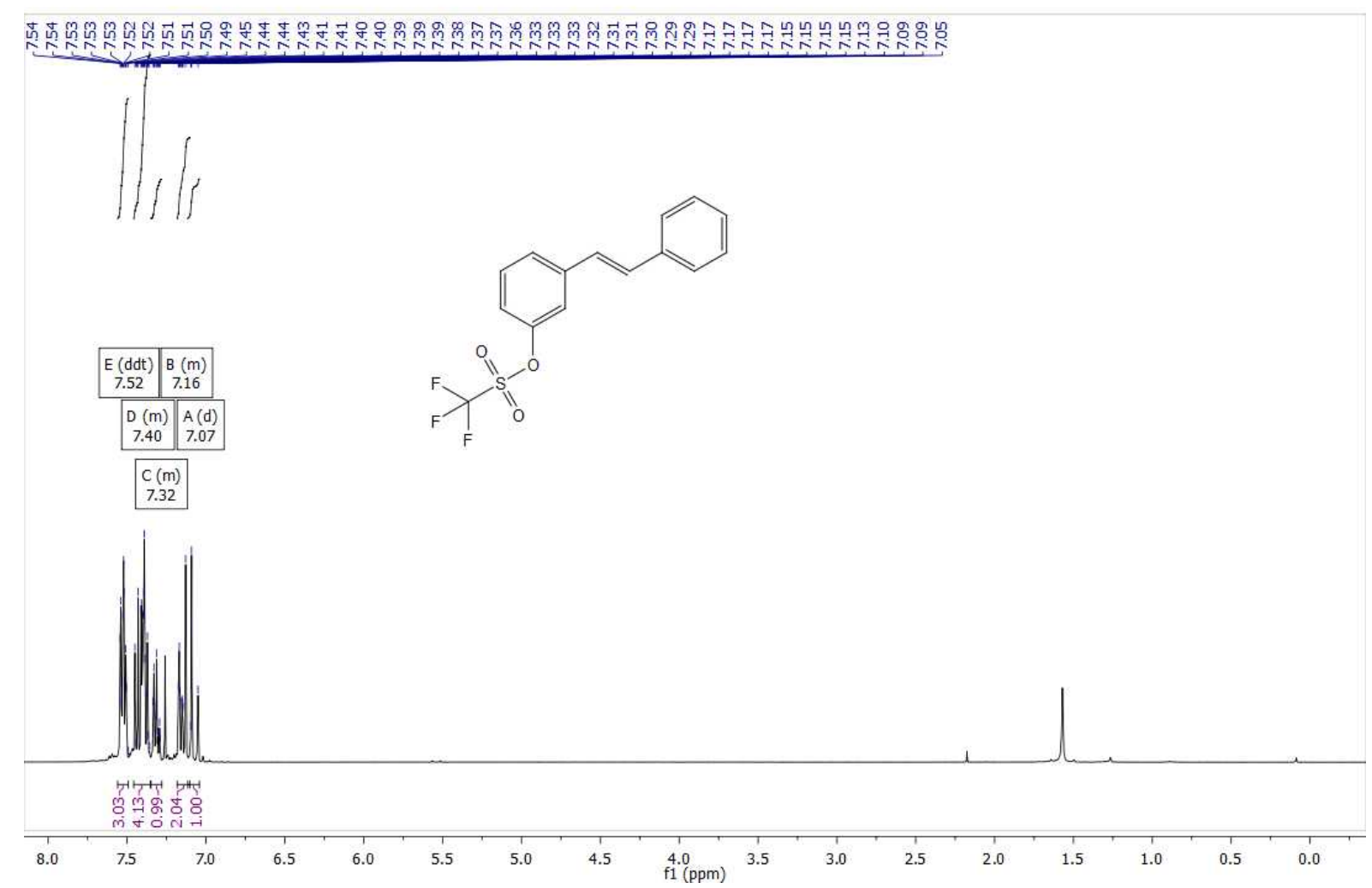

(E)-3-styrylphenyl trifluoromethanesulfonate

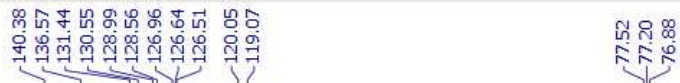
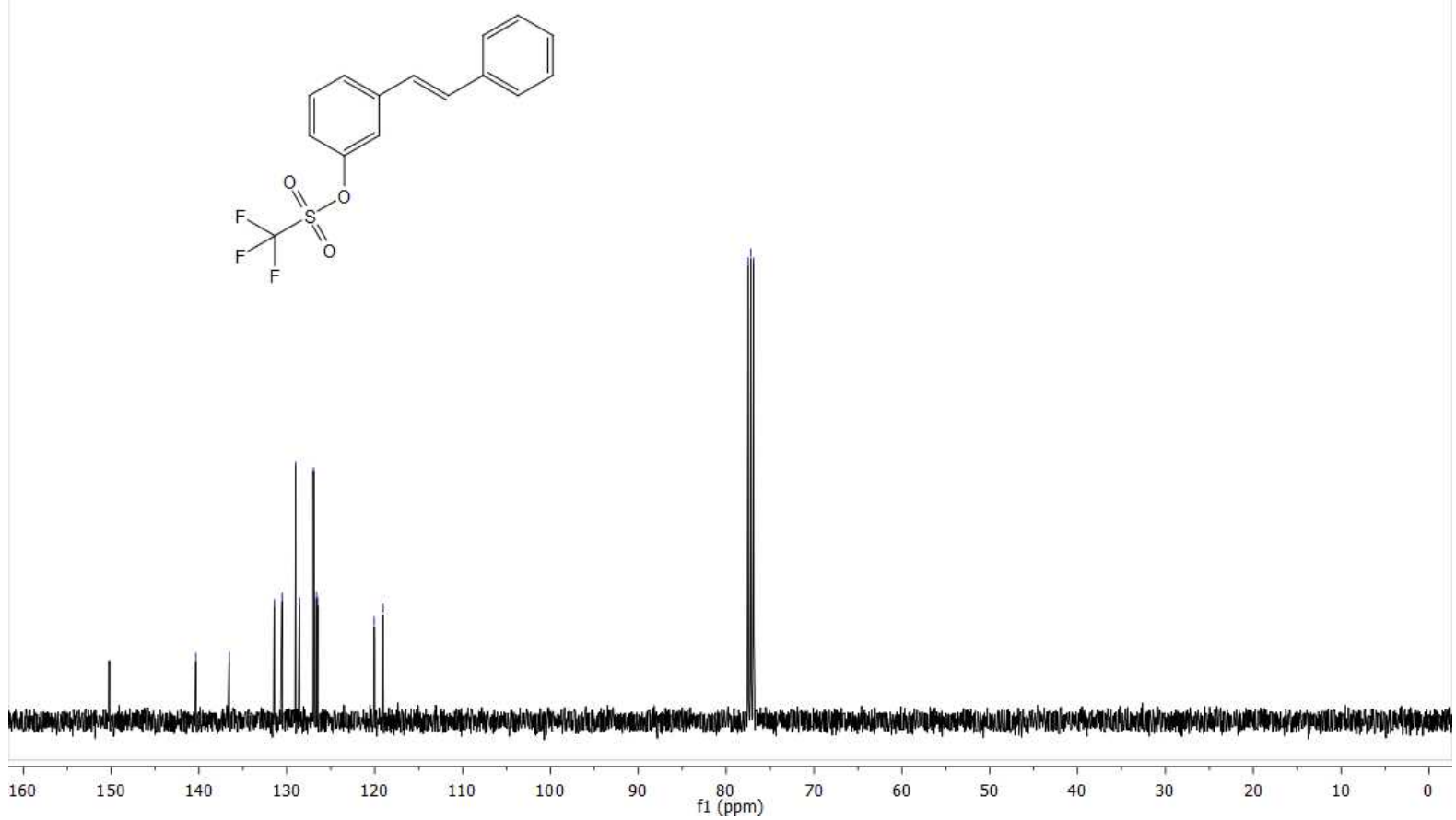


\section{(E)-3-styryl-1,1'-biphenyl (7a):}

(E)-3-styryl-1,1'-biphenyl

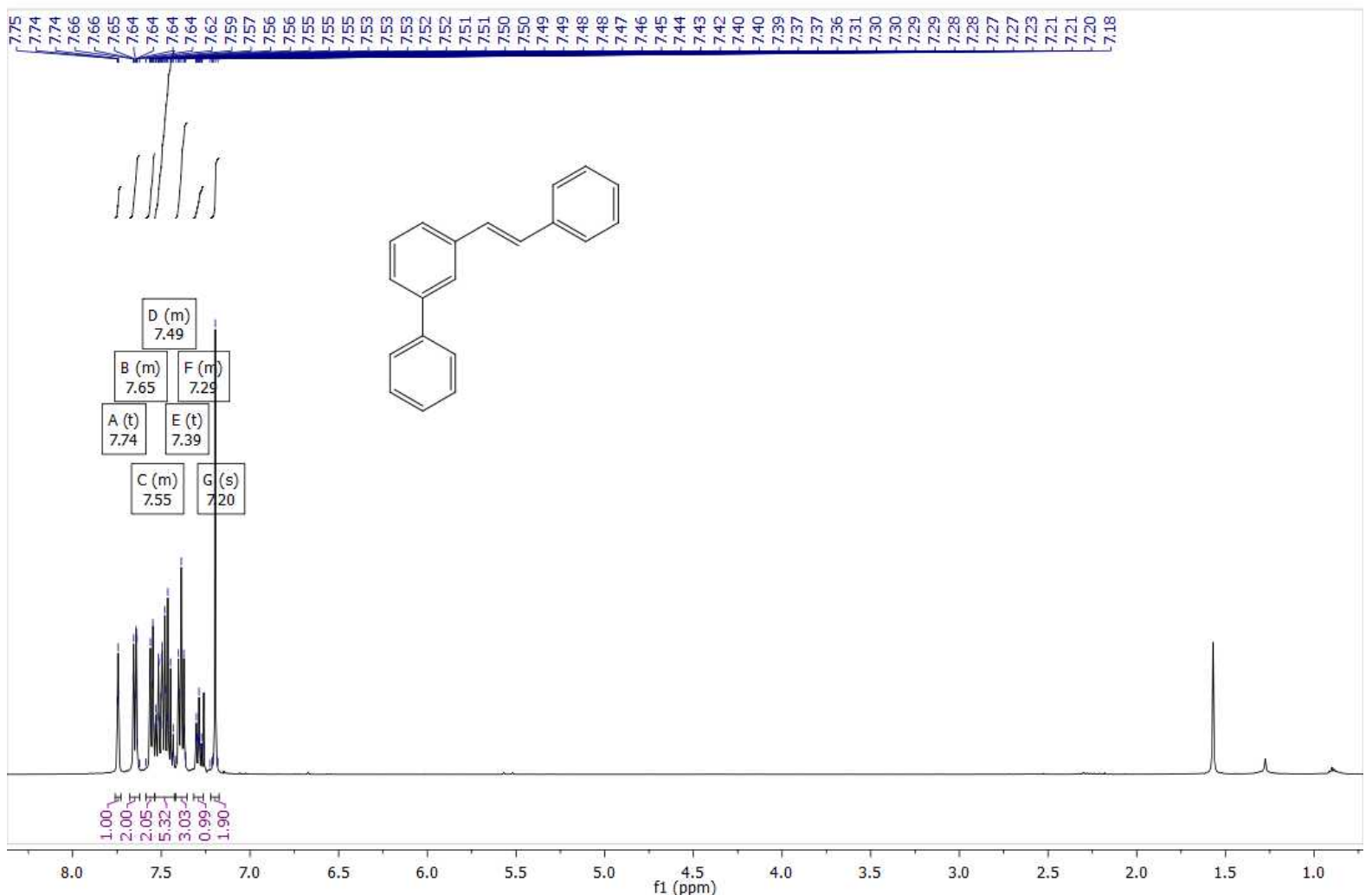

(E)-3-styryl-1,1'-biphenyl

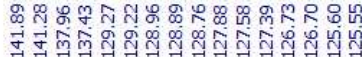

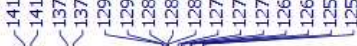
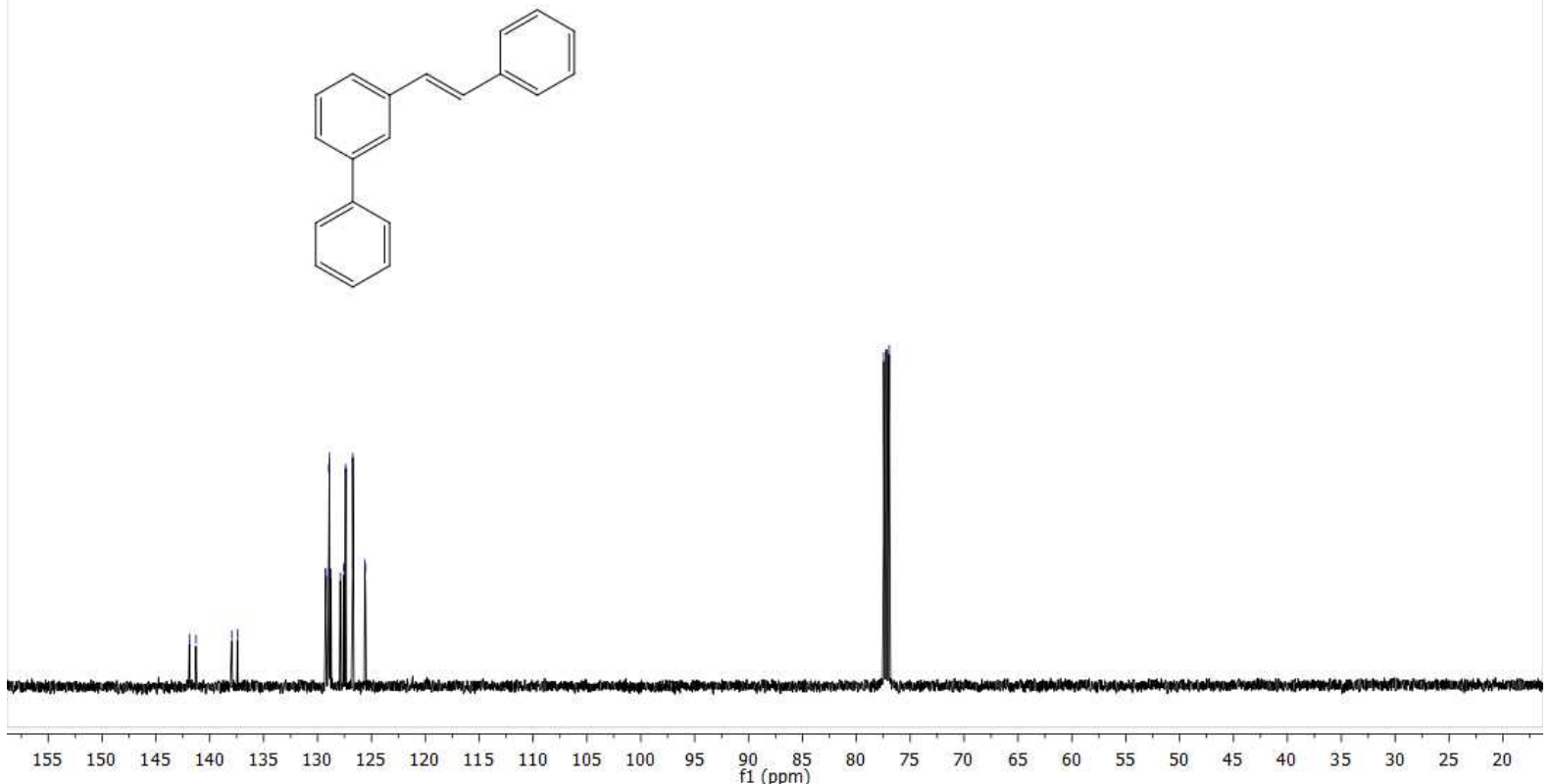
(E)-N-phenyl-3-styrylaniline (7b):

(E)-N-phenyl-3-styrylaniline

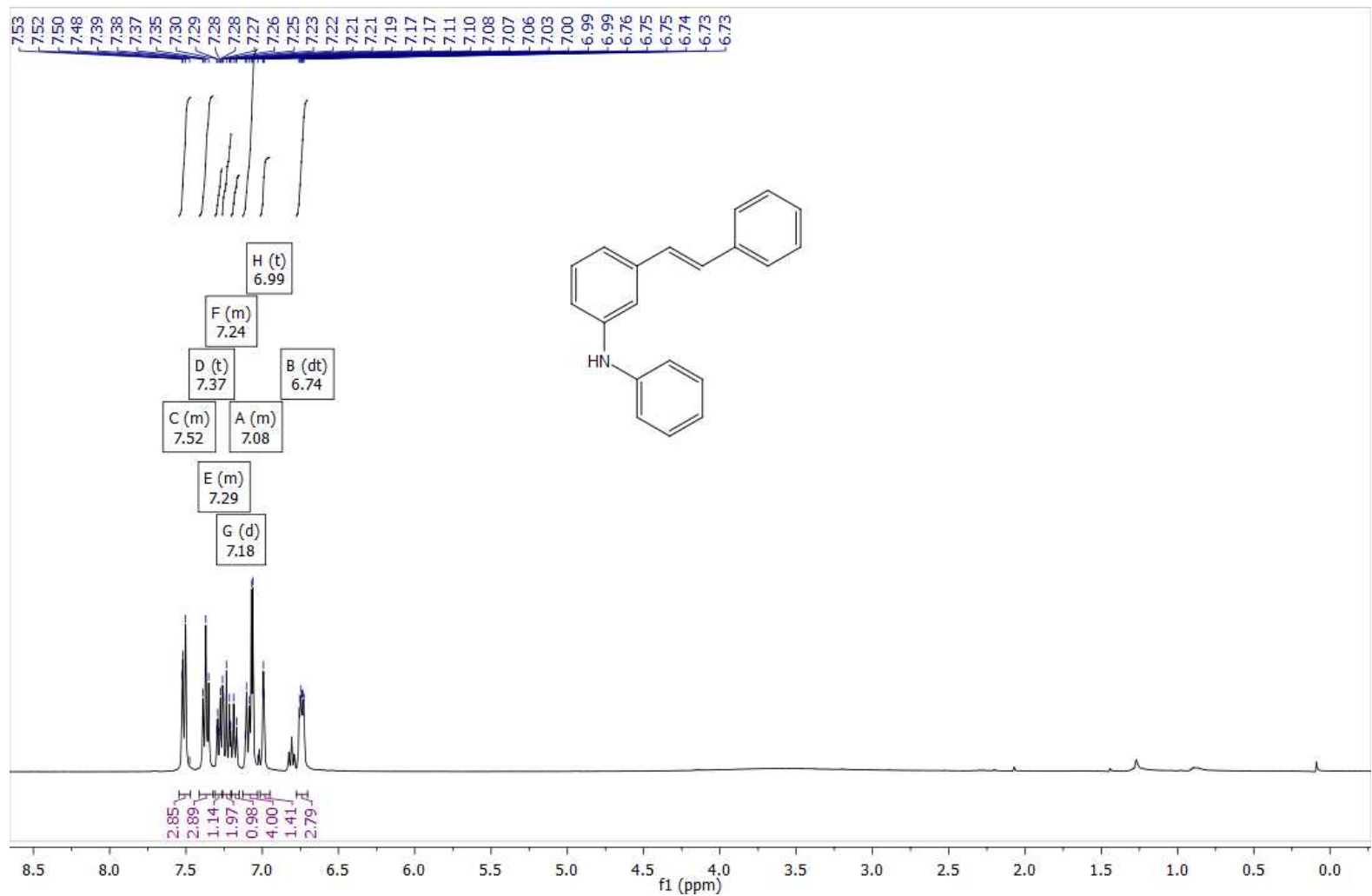

(E)-N-phenyl-3-styrylaniline

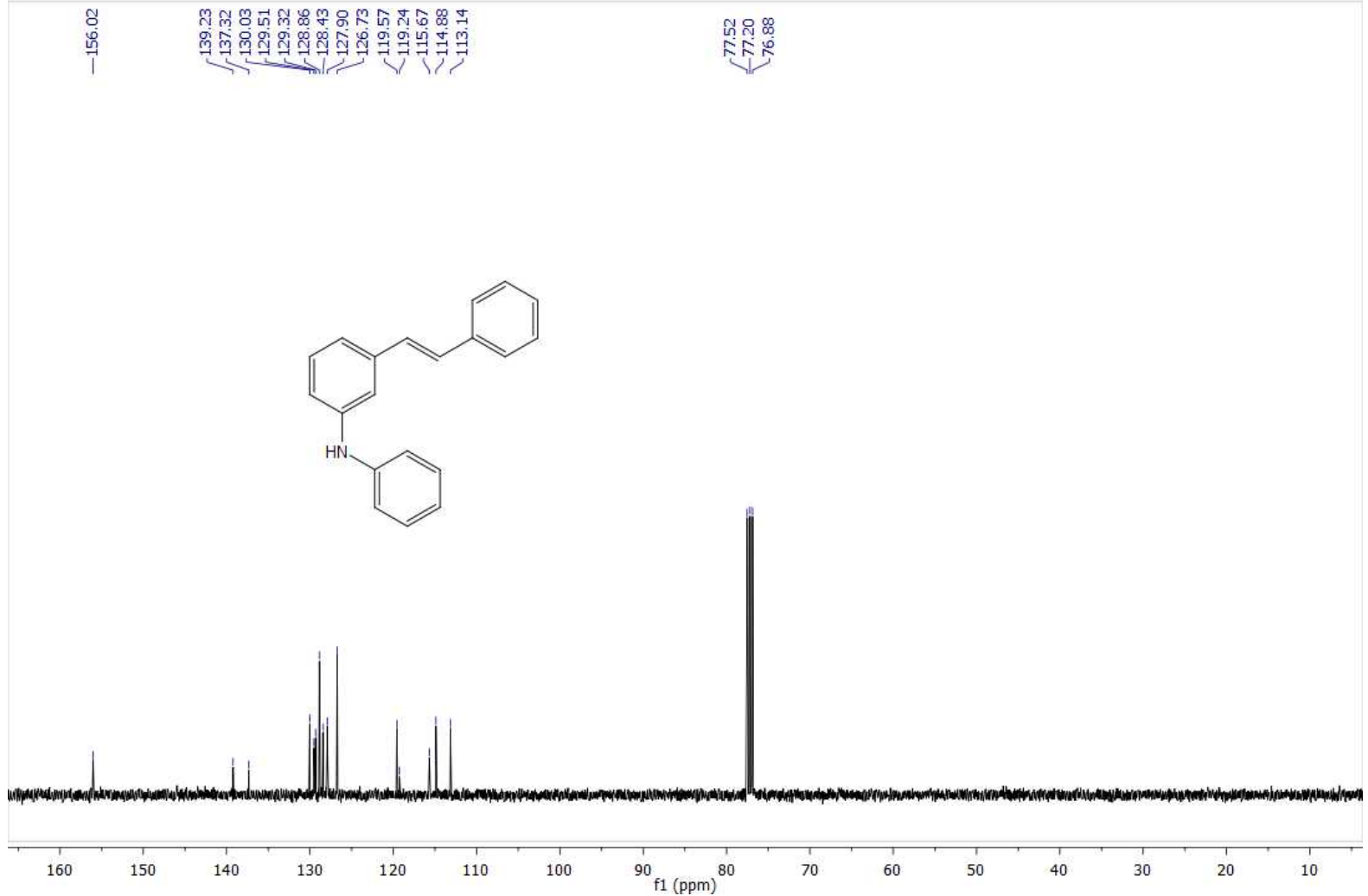




\section{(E)-1-styryl-3-(p-tolylethynyl)benzene (7c):}

(E)-1-styryl-3-(p-tolylethynyl)benzene

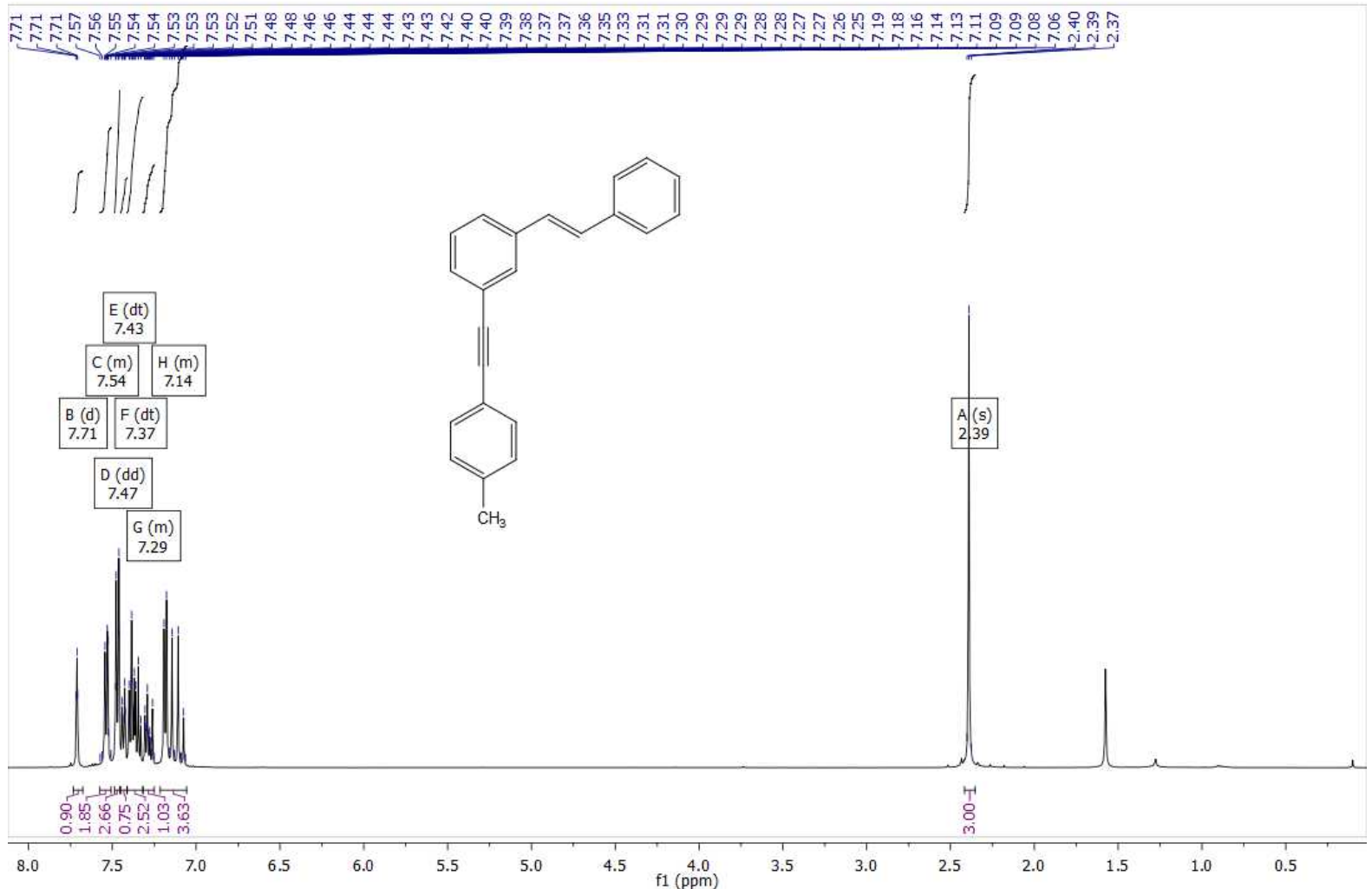

(E)-1-styryl-3-(p-tolylethynyl)benzene

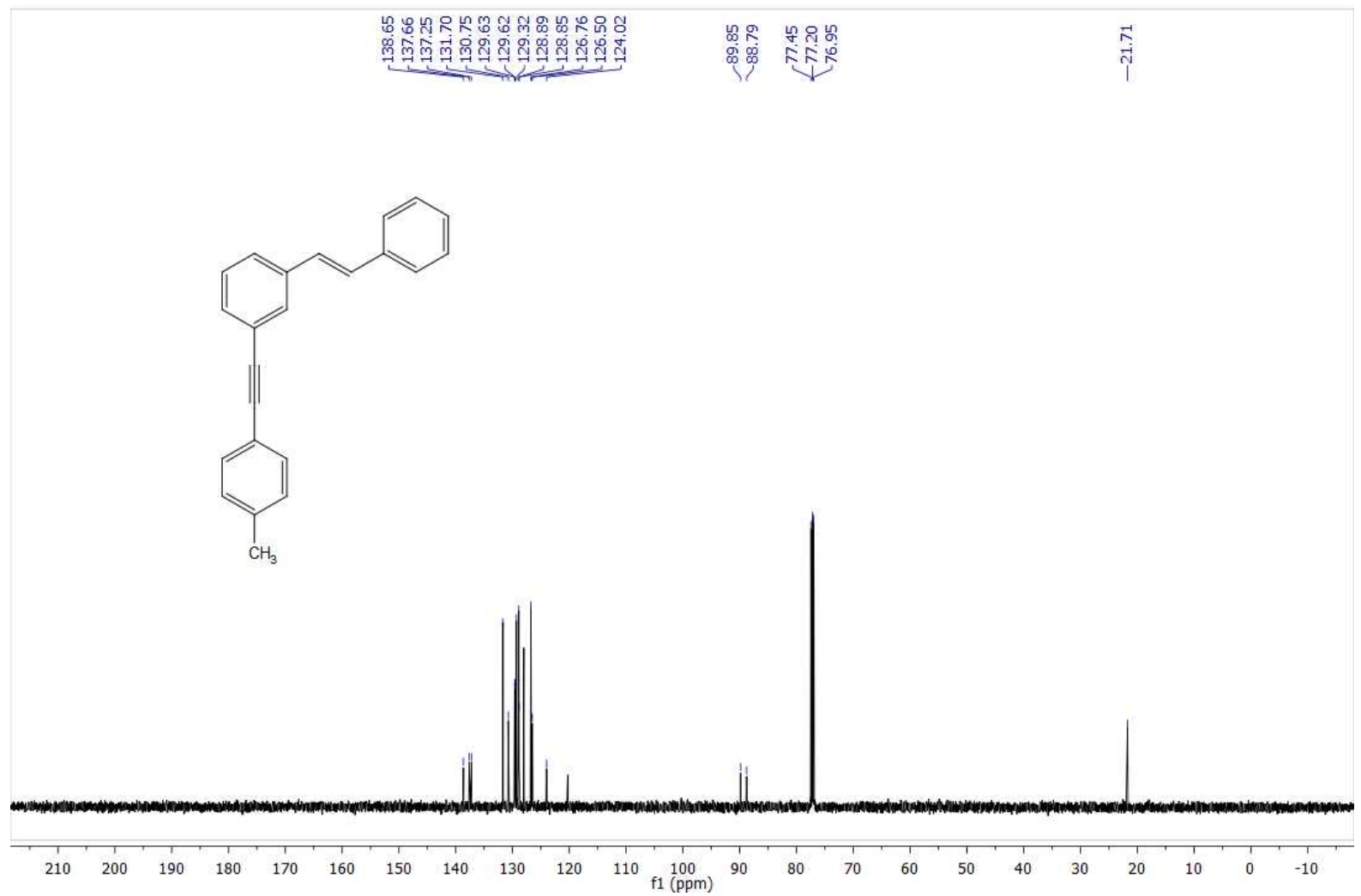


<smiles>[2H]c1c([2H])c([2H])c(C([2H])([2H])P(=O)(OCC)Oc2ccccc2C#N)c([2H])c1[2H]</smiles>

[D글 Benzylphosphonate ester (1a-D):

${ }^{1}$ H NMR:

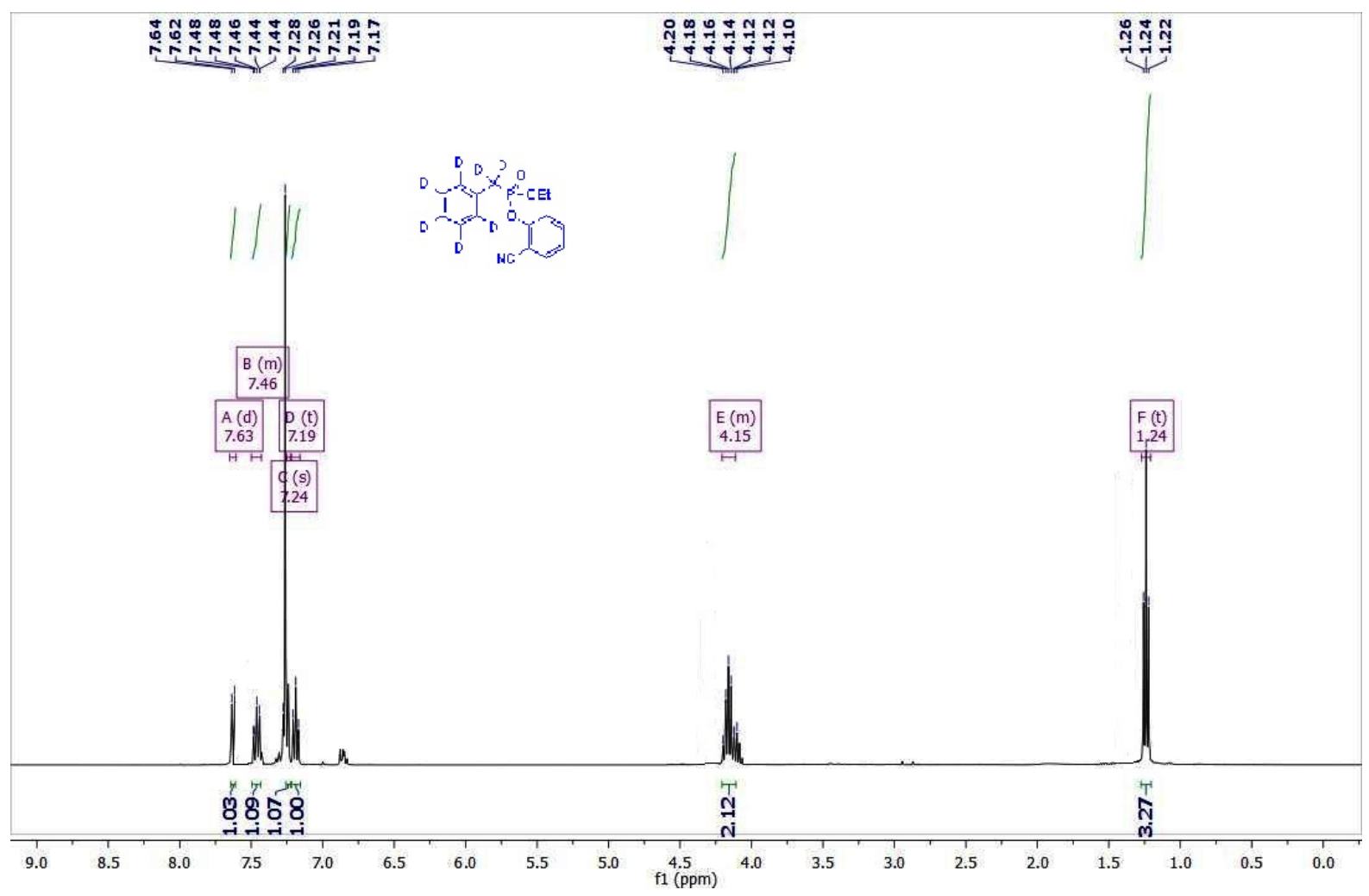


${ }^{13}$ C NMR:

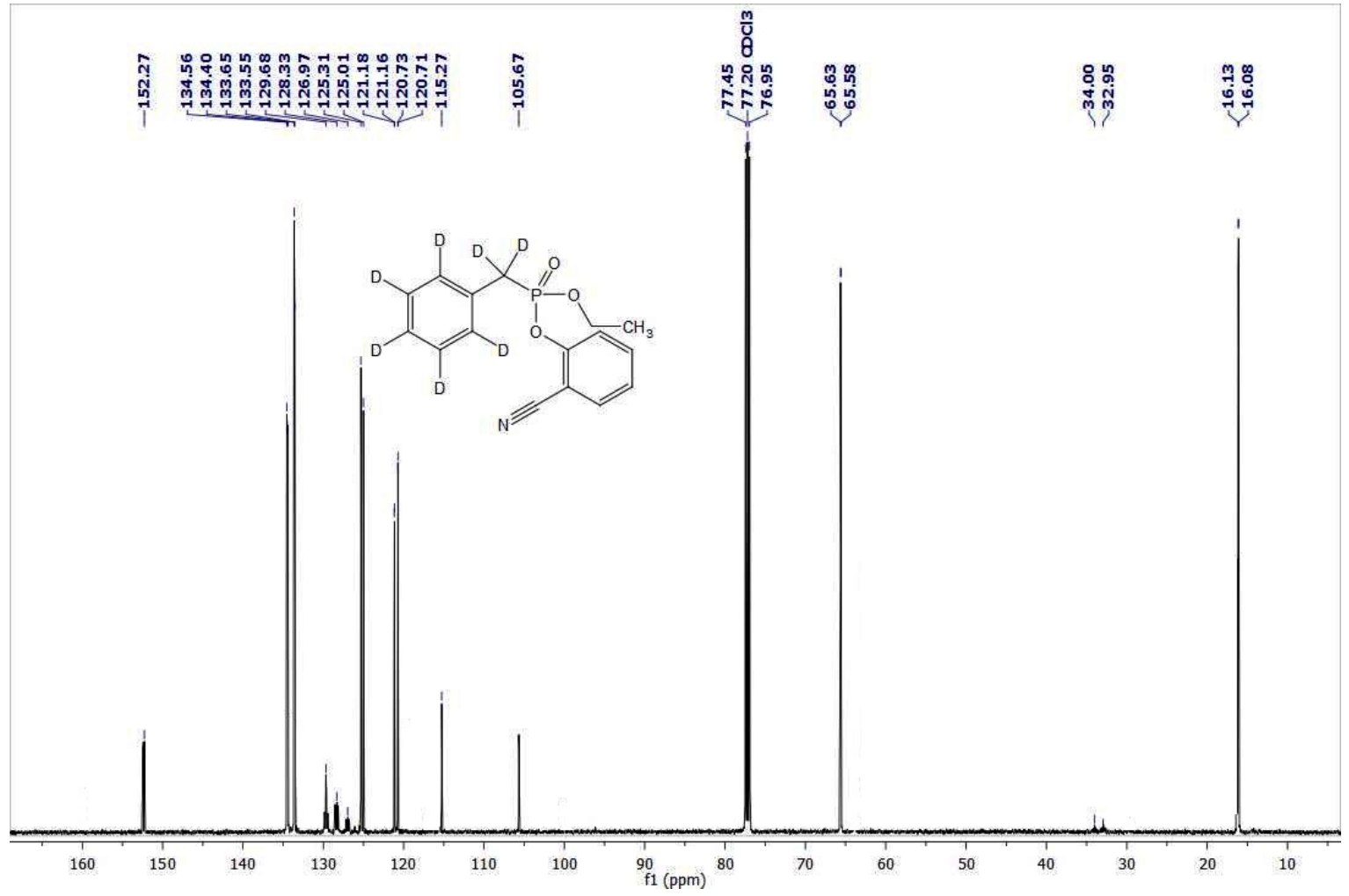

Aromatic region (aromatic C-D triplet and multiplet):

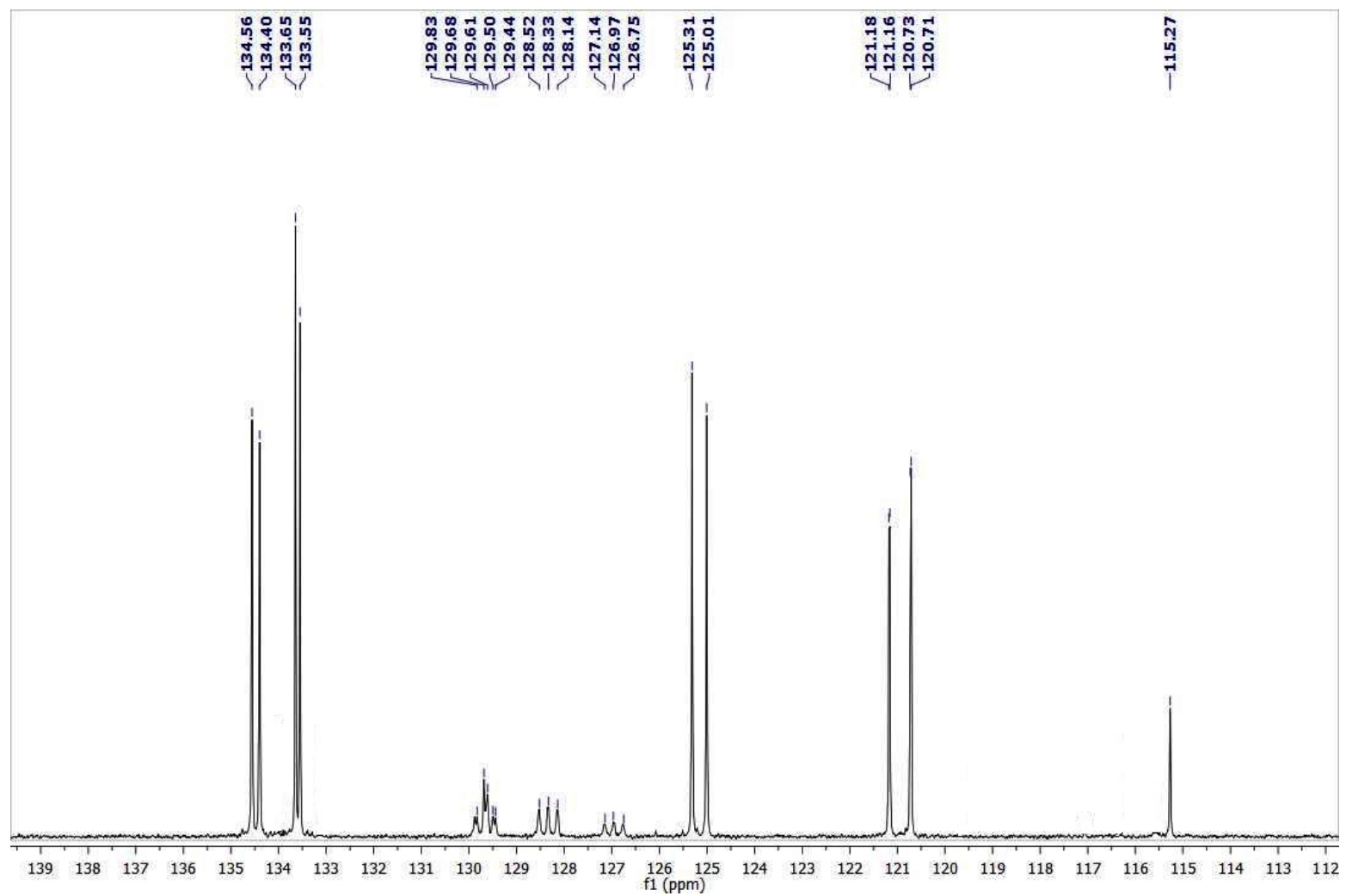


Aliphatic $\mathrm{CD}_{2}$ multiplet:

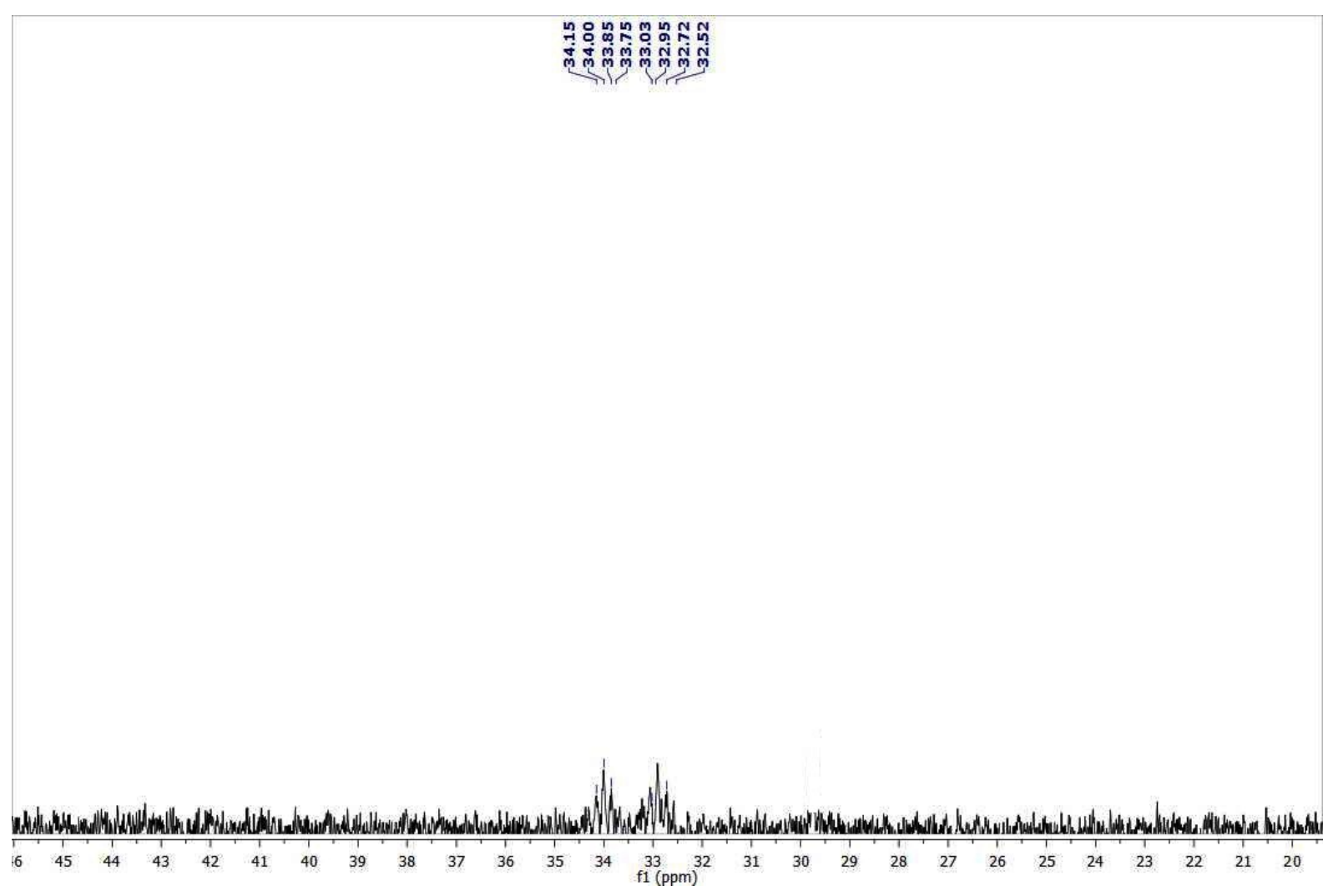


Intermolecular KIE experiment \{product distribution $\left[\mathbf{P}_{\mathbf{H}} / \mathbf{P}_{\mathbf{D}}\right]$ spectrum\}:

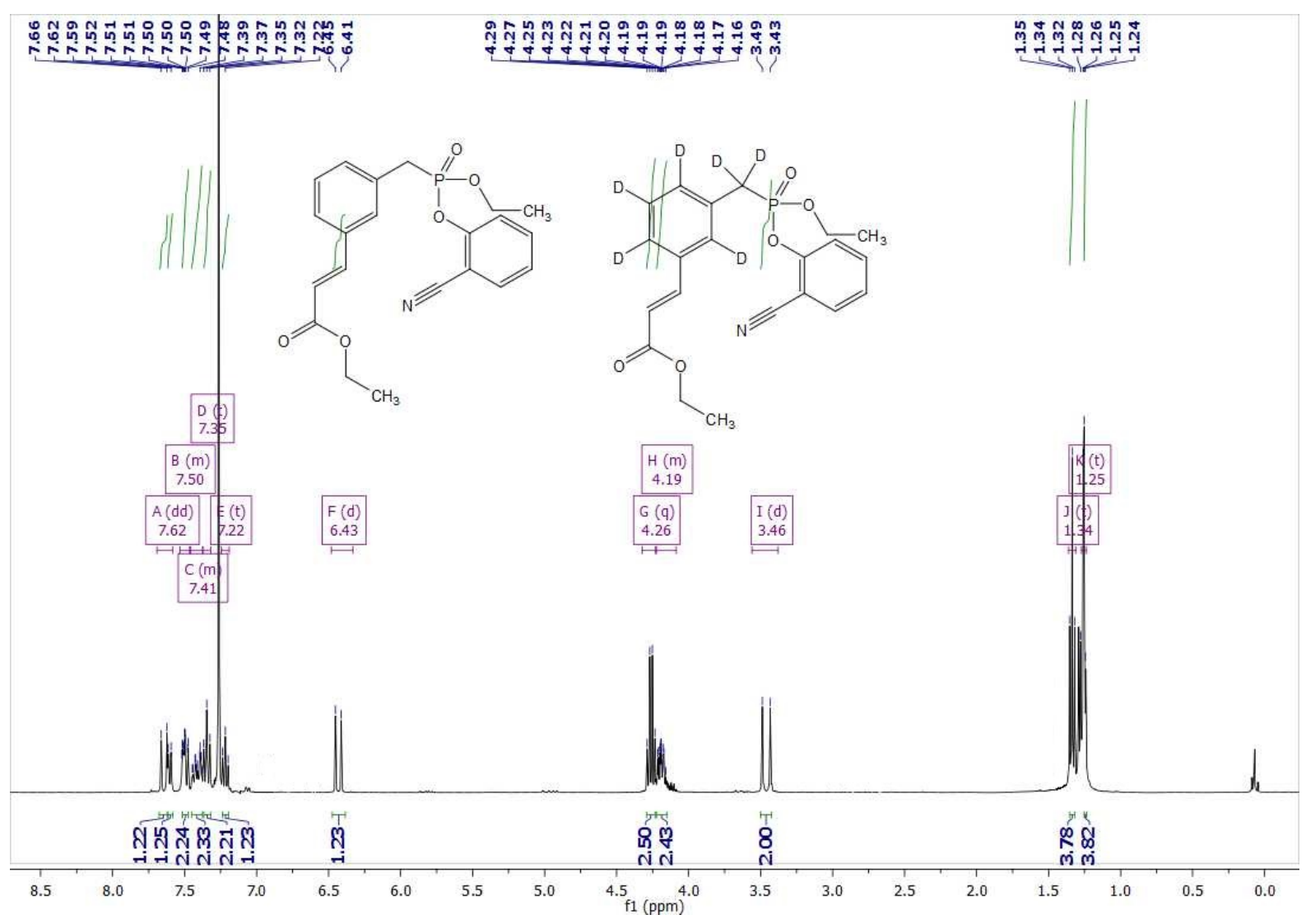

In this spectrum one $\mathrm{C}-\mathrm{H}$ proton integration coming from compound $2 \mathrm{a}$ is " 1 " and one $\mathrm{C}-\mathrm{H}$ proton integration coming from compound $2 \mathrm{a}-\mathrm{D}$ is " 0.23 ".

$\left[\mathbf{P}_{\mathrm{H}} / \mathbf{P}_{\mathrm{D}}\right]=\mathbf{1 / 0 . 2 3}=4.5$ 
<smiles>CCOCC=Cc1cc(CP(=O)(OCC)Oc2ccccc2C#N)ccc1C(C)(C)C</smiles>

(E)-ethyl3-(2-tert-butyl-5-(((2-cyanophenoxy)(ethoxy)phosphoryl)methyl)phenyl)acrylates (2o):

\section{${ }^{1}$ HNMR:}

(E)-ethyl 3-(2-tert-butyl-5-(((2-cyanophenoxy)(ethoxy)phosphoryl)methyl)phenyl)acrylate

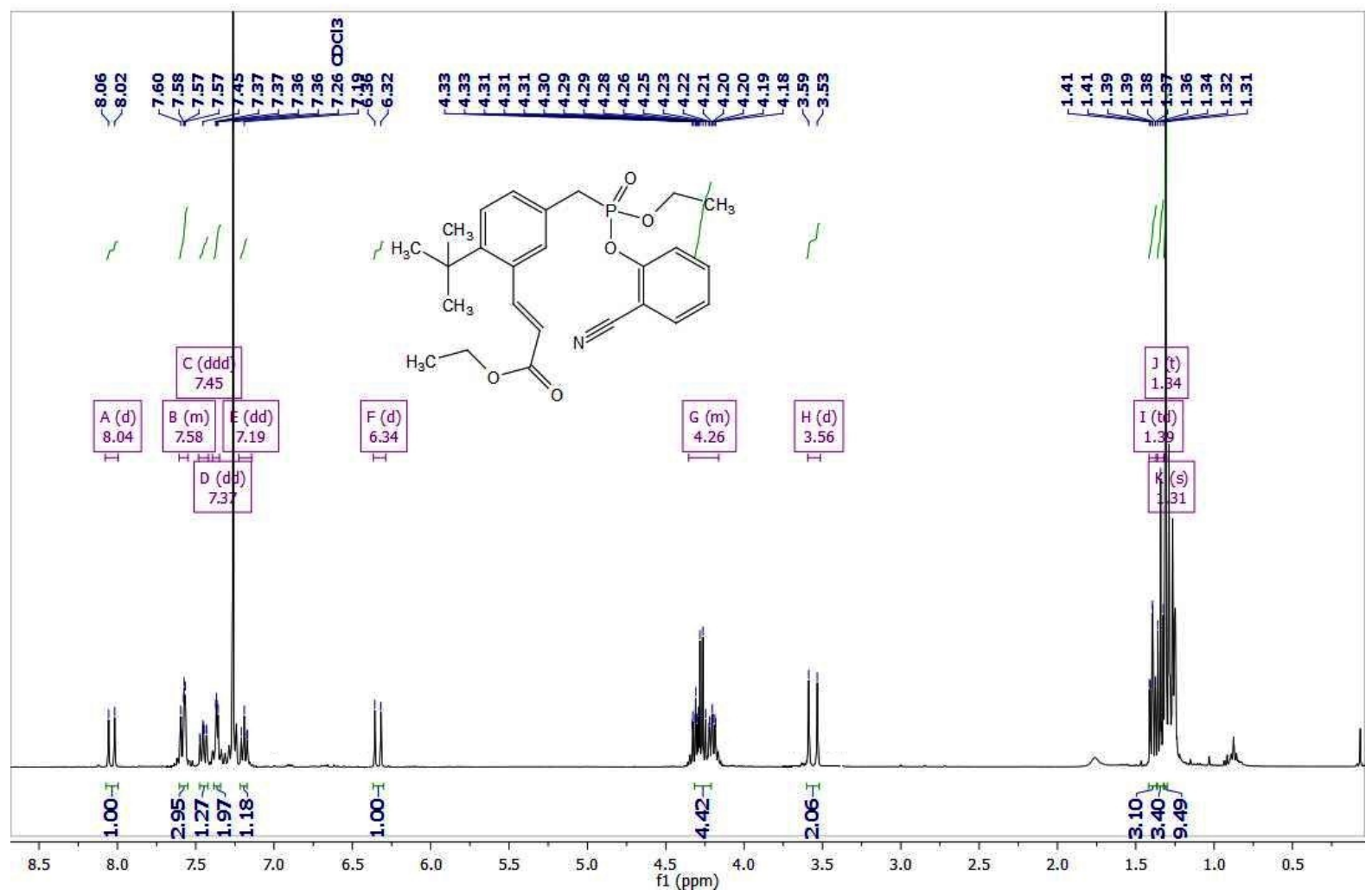


${ }^{13}$ C NMR:

(E)-ethyl 3-(2-tert-butyl-5-(((2-cyanophenoxy)(ethoxy)phosphoryl)methyl)phenyl)acrylate

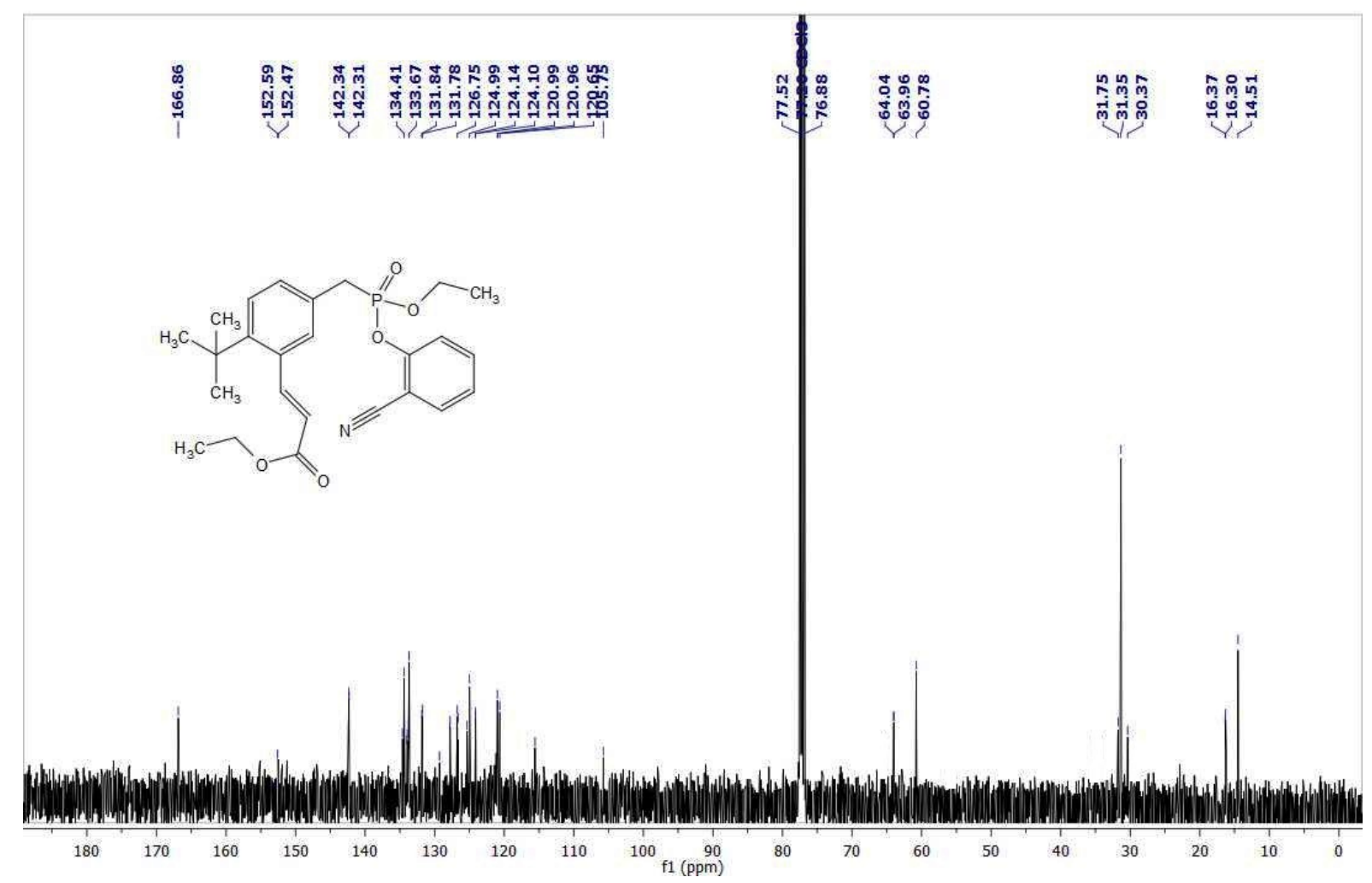

